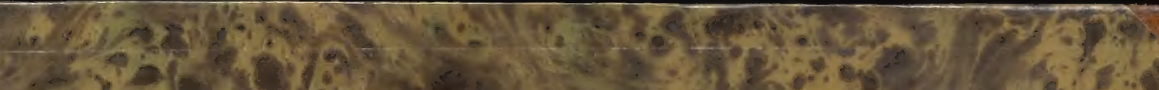

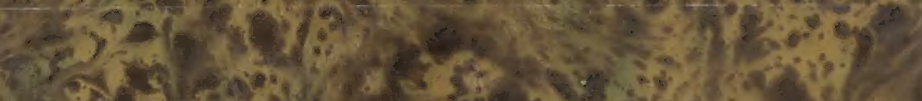

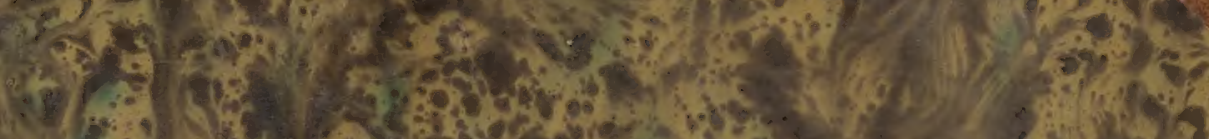

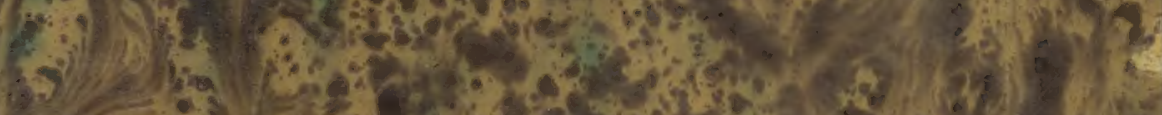

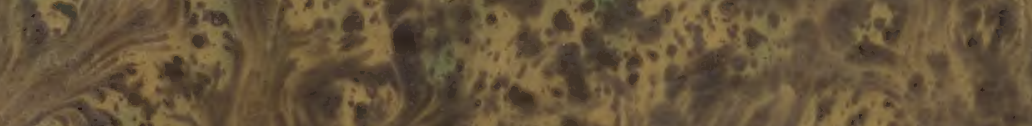

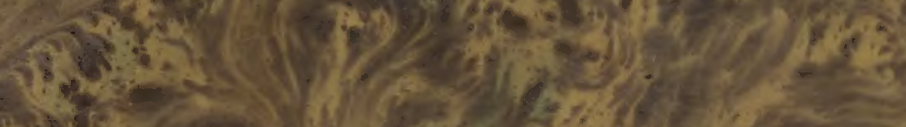

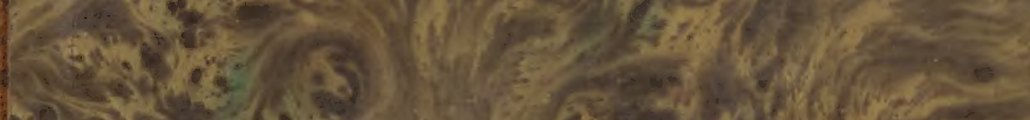

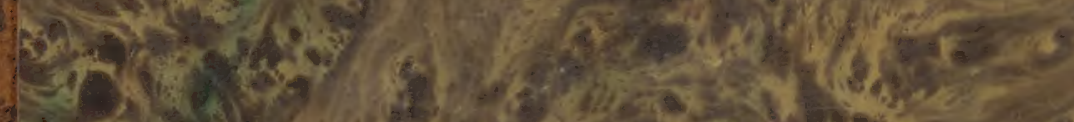

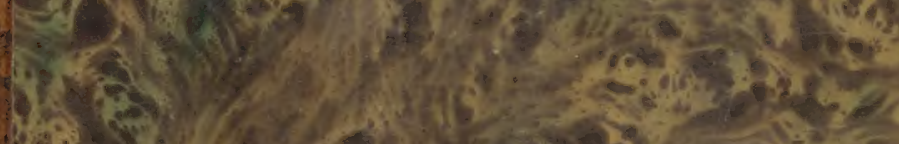

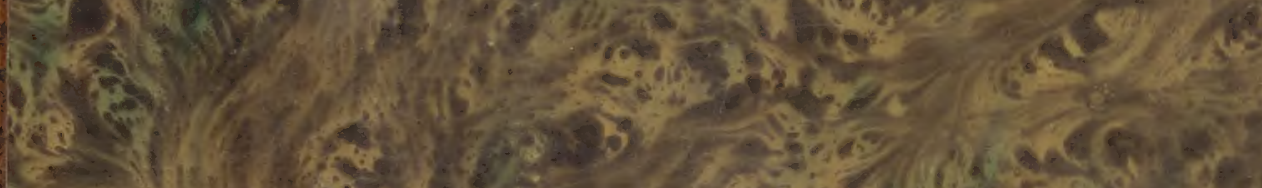

tas a

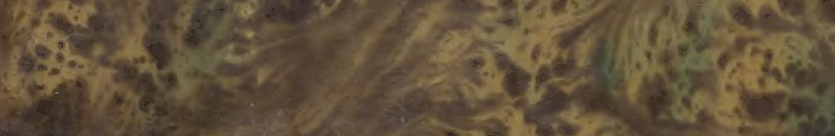

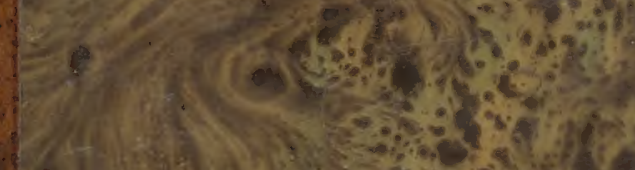

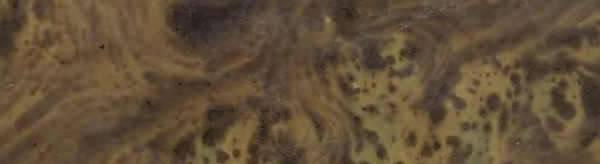

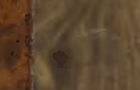

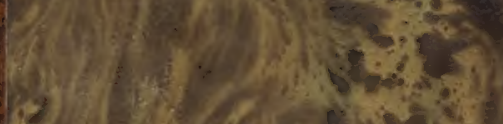

(a)

13 


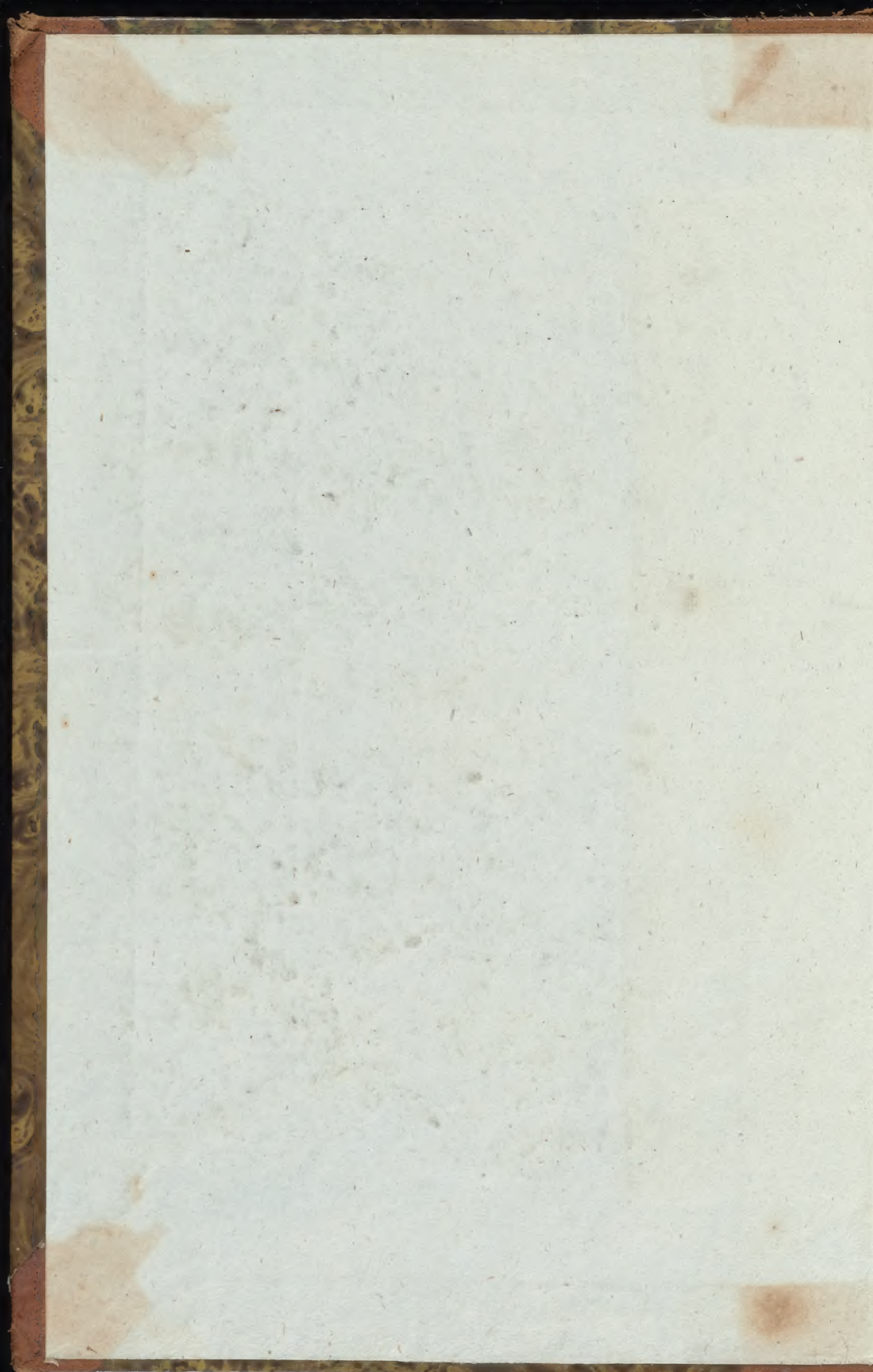



$x^{2}+20$ 
HISTOIRE ET DESCRIPTION

DU

MUSÉUM ROYAL

D'HISTOIRE NATURELLE. 
L'éditeur, s'étant conformé aux lois et ordonnances sur la librairie, fera saisir tout exemplaire qui ne serait pas revêtu de sa signatutes 


\section{HISTOIRE ET DESCRIPTION}

\section{DU \\ MUSÉUM ROYAL D'HISTOIRE NATURELLE,}

DUYRAGE RÉDIGÉ D'APRÉS LES ORDRES DE L'ADMINISTRATION DU MUSÉUM,

\section{PAR M. DELEUZE,}

Avec trois plans et quatorze vues des Jardins, des Galexies et de la Ménagerie.

\section{A PARIS,}

CHEZ M. A. ROYER, AU JARDIN DU ROT.

DE L'TMPRIMERTE DE L. T. GELLOT, RUE DU COLOMBIER, $N^{\circ} 30$.

M. DCCC. XXIII.

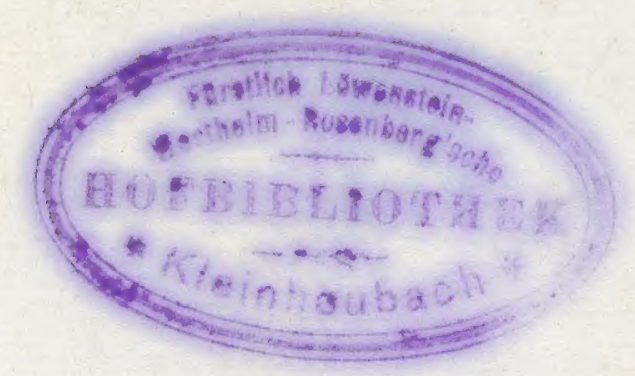


K

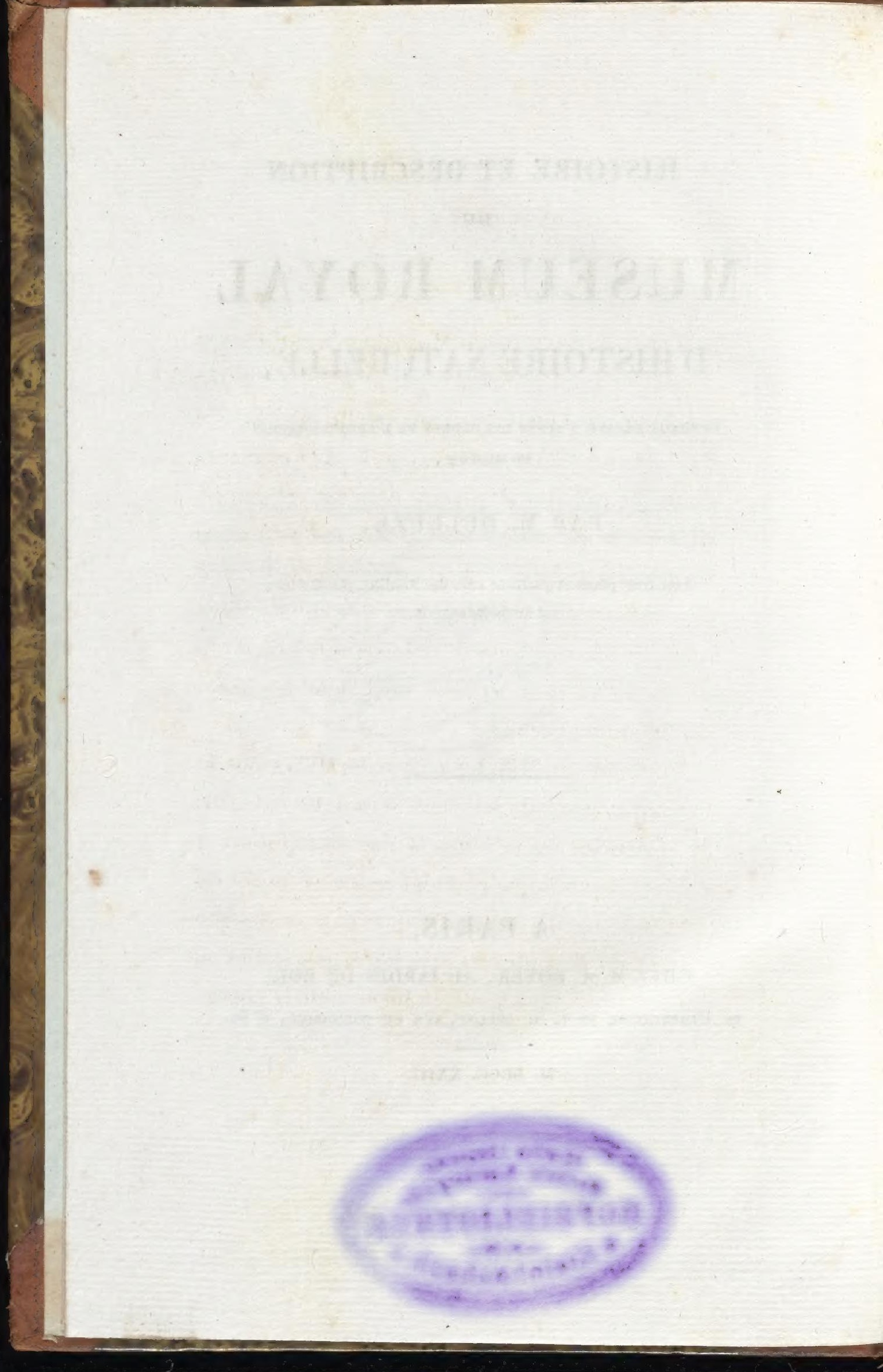




\section{PRÉFACE.}

L'Administration du Muséum m'ayant chargé de rédiger une histoire abrégée et une description succincte de l'établissement qu'elle dirige, j’ai dû faire mes efforts pour répondre à la confiance dont elle m'honorait; mais il m'eût été impossible de remplir cette tâche, si MM. les professeurs ne m'eussent donné, chacun pour sa partie, soit des instructions verbales, soit des notes écrites, selon que le sujet que je devais traiter avait plus ou moins de rapport avec ceux dont je me suis spécialement occupé.

Plusieurs de MM. les aide-naturalistes ont eu la complaisance de coopérer à mon travail. Ainsi M. Valenciennes m'a remis une description du cabinet de zoologie pour les animaux vertébrés, pour une partie des mollusques et pour les zoophytes, et M. Dufresne y a joint une notice sur les coquilles. M. Latreille a bien voulu se charger du tableau des crustacés, des arachnides et des insectes; et M. de La Fosse, qui suppléait dans 
ses leçons le célèbre professeur dont le Muséum déplore la perte, m'a donné l'article de la minéralogie. M. Frédéric Cuvier m'a fourni pour la Ménagerie tous les renseignemens dont j'avais besoin. Enfin, c'est à M. Laurillard que je dois les détails relatifs à la collection d'anatomie comparée et à celle des ossemens fossiles. Je prie ces messieurs de recevoir l'expression de ma reconnaissance.

M. Royer, employé dans les bureaux de l'administration, s'est chargé de tous les frais de l'édition, et il a fait dessiner et graver les plans et les vues de l'établissement.

Quoique cet ouvrage ne soit qu'une simple esquisse, j'espère qu'en donnant une idée des richesses du Muséum, il fera connaître son importance et son utilité. Puisse-t-il rappeler à tous les Français les obligations que les sciences naturelles ont à nos Rois, qui, depuis Louis XIII jusqu'à Louis XVIII, n'ont cessé d'en encourager l'étude et d'en faciliter les progrès. 


\section{HISTOIRE ET DESCRIPTION}

\section{DU MUSEUM}

\section{D'HISTOIRE NATURELLE.}

\section{INTRODUCTION.}

Parmi les établissemens consacrés à l'instruction publique, le Muséum d'histoire naturelle est certainement l'un des plus dignes d'admiration, l'un de ceux qui présentent le plus d'utilité. Toutes les sciences naturelles, ainsi que les applications qu'on peut faire de ces sciences y sont également enseignées : il offre pour chacune d'elles les collections les plus nombreuses. L'ordre dans lequel tout y est disposé, facilite l'étude, donne le moyen de comparer les êtres, et fait qu'on peut trouver 
à l'instant ce qu'on cherche. Des professeurs, choisis parmi les savans les plus distingués de la France, enseignent à observer, à connaître, à classer les êtres naturels, non point en exposant simplement des théories, ou en donnant des descriptions auxquelles la lecture d'un bon ouvrage pourrait suppléer, mais en mettant sous les yeux des élèves les objets sur lesquels leurattention doit se fixer. Au sortir des leçons, on peut aller, soit dans l'école de botanique, où sont réunies toutes les plantes vivantes, et dans la ménagerie, où l'on nourrit plusieurs animaux rares, soit dans les galeries de zoologie et de botanique, où l'on conserve desséchés ou dans l'esprit-de-vin tous les êtres organiques que l'on a pu se procurer depuis des siècles, étudier les familles et les genres, comparer les espèces, examiner les détails, et se familiariser avec les caractères. Après les cours de minéralogie et de géologie, de riches collections arrangées méthodiquement et sous divers points de vue, offrent les mêmes ressources. On peut enfin, dans le cours d'anatomie comparée, s'inslruire de l'organisation des animaux; dans ceux de chimie, de la composition des corps et de l'usagr qu'on en fait pour les arts; dans celni 
de culture, des procédés les plus convenables pour élever, soigner et naturaliser les végétaux utiles; dans celui d'iconographie, de l'art de dessiner les objets d'histoire naturelle, de manière à en exprimer les caractères distinctifs. Ceux qui étudient, ayant souvent besoin de chercher le développement des principes que le professeur leur a donnés, ou de comparer leurs propres observations, à celles qui ont été faites avant eux; une bibliothèque uniquement consacrée à l'histoire naturelle, et qui est ouverte tous les jours, offre les livres qu'il est utile de consulter, ceux qui contiennent l'histoire de la science, la description ou la figure des objets connus, et l'état actuel de nos connaissances.

Comme toutes les sciences naturelles sont liées, les professeurs ont entre eux des communications qui les mettent à même d'étendre leurs vues au delà des objets dont ils se sont spécialement occupés, et la réunion des observations et des découvertes propres à chacune des branches de l'histoire naturelle, forme un tout qui est vraiment la philosophie de la nature.

Le savant vient au Muséum augmenter ses con- 
naissances et recueillir des notions positives sur le sujet qu'il veut approfondir; l'homme méditatif y contemple un ensemble de merveilles, il y admire la richesse de la nature, et la puissance du Créateur qui a donné à la nature des lois invariables; le jeune homme né pour l'étude, s'enflamme en pensant aux savans illustres qui s'y sont succédés, et dont tout lui rappelle les travaux et la gloire; le curieux s'extasie à la vue d'un nombre prodigieux de végétaux et d'animaux étrangers, à l'aspect de l'éclat des collections; l'homme qui se livre aux lettres ou aux beaux-arts, y trouve la source d'une infinité d'idées, et des modèles en tout genre.

Au milieu de ce spectacle imposant, l'activité des travaux, la variété des plantations, le parfum des fleurs, la beauté des allées; la vue qui du haut d'une colline plantée d'arbres verds, s'étend sur la ville et sur la campagne; le grand nombre d'amateurs qui viennent jouir de la promenade et semblent oublier les agitations du monde : tout concourt à faire du Muséum un séjour de paix pour l'âme, et de ravissement pour l'esprit.

Les hommes livrés à l'étude d'une des bran- 
ches de I'histoire naturclle, connaissent bientôt la partie de l'établissement qui doit essentiellement les intéresser : mais après une assiduité de quelques années, ils ne pourraient épuiser les richesses des autres parties. Les étrangers et les curieux qui ont des devoirs à remplir, des affaires à suivre et peu de temps dont ils puissent disposer, demandent qu'on les dirige dans leurs recherches, et qu'on leur fasse remarquer ce qui doit principalement arrêter leurs regards. C'est dans l'intention de leur être utile que nous allons donner une description abrégée de toutes. les parties de l'établissement. Mais avant de conduire nos lecteurs à ce qu'il y a de plus digne d'attention, nous croyons devoir leur faire connaître l'établissement en lui-même, sa fondation, ses accroissemens successifs, son administration ancienne, le nom des savans qui y ont été employés et qui ont contribué à son lustre, sa nouvelle organisation et son état actuel. Cette notice sera fort succincte. Ceux qui désireront plus de détails peuvent lire les Mémoires de M. de Jussieu, insérés dans les Annales du Muséum, tom. I, 2, 3, 4, 6 et I I ( I), et l'Histoire du Muséum, donnée en allemand par Fischer. 
Le nom de Muséum d'histoire naturelle est récent : il date de l'époque de la nouvelle organisation : il est employé à désigner la réunion de trois établissemens principaux, le jardin du Roi, le cabinet du Roi, et la ménagerie du Roi : car, dans une monarchie, les établissemens consacrés à la prospérité nationale, et payés par le gouvernement, sont sous la dépendance et la proteçtion immédiate du Roi. Nous allons voir comment ces trois établissemens ont été successivement fondés et réunis.

(1) Ces Mémoires ne vont que jusqu’à la mort de Buflon, en $1-88$. 



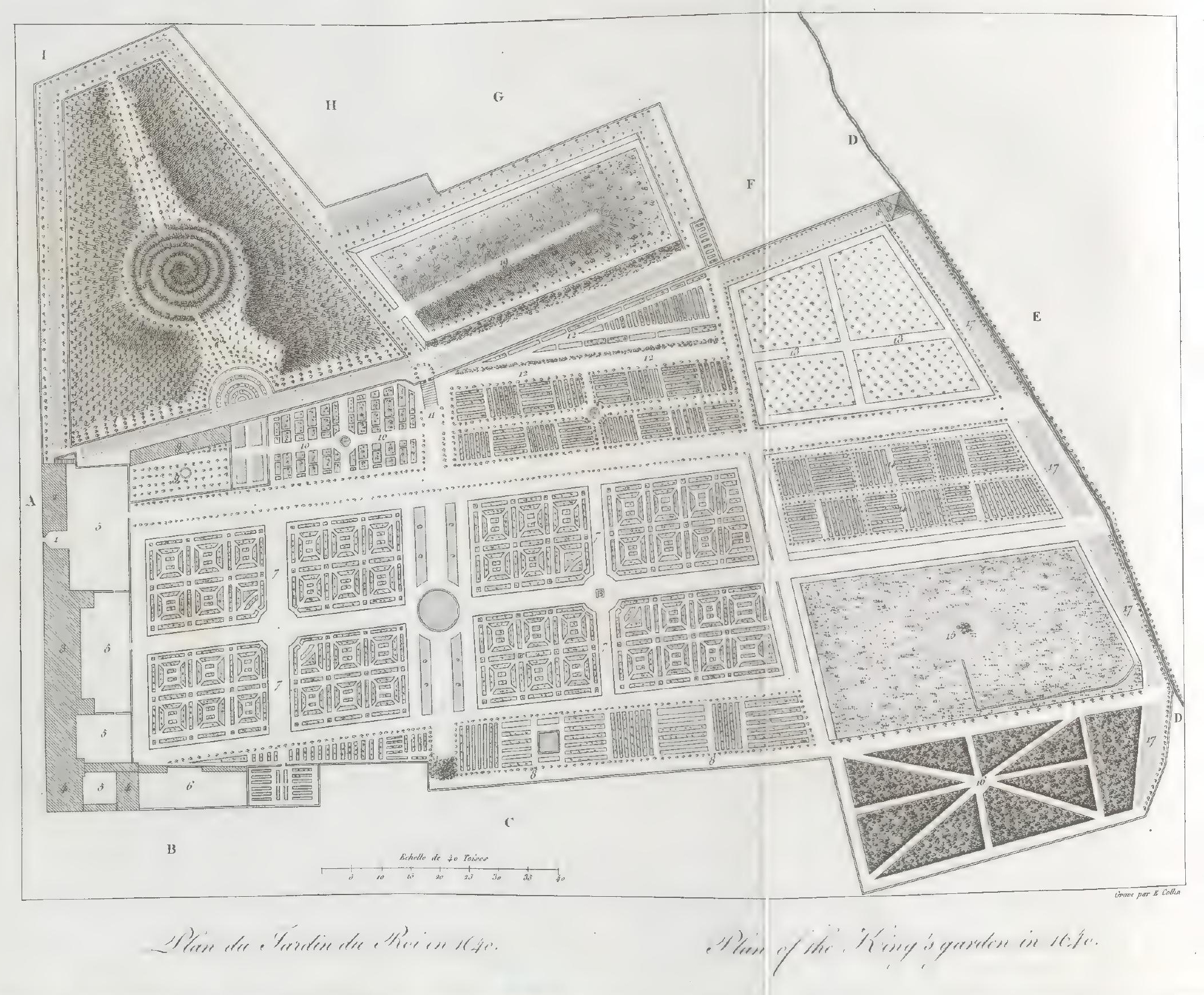




\section{HISTOIRE}

\section{DU MUSÉUM.}

PREMIÈRE ÉPOQUE.

DEPUIS LA FONDATION JUSQU'EN 1739.

LE Jardin du Roi fut créé par Louis XIII, qui l'institua et l'organisa par un édit donné et enregistré au parlement, au mois de mai 1635.

Neuf ans auparavant, Louis XIII, à la sollicitation d'Hérouard, son premier médecin, et de Guy de La Brosse, son médecin ordinaire, avait autorisé par lettres patentes la fondation du jardin, et l'acquisition d'une maison et d'un terrain de 24 arpens dans le faubourg Saint-Victor, et dans le même local qui fait aujourd'hui partie du Muséum. Il avait donné la surintendance de cet établissement à son premier médecin et à ses suc- 
cesseurs, avec pouvoir de choisir un intendant qui résiderait dans le jardin et en aurait la direction. Hérouard avait choisi Guy de La Brosse, et ce choix avait été ayréé par le Roi. Mais la mort d'Hérouard, 'et d'autres circonstances ayant retardé l'exécution des lettres patentes, la fondation du Jardin ne date réellement que de 1635.

L'édit donné à cette époque, ratifie l'acquisition de la maison et du terrain ; il nomme Bouvard, alors premier médecin du roi, surintendant, et Guy de La Brosse, intendant du Jardin. Il contient. enfin tout ce qui est relatif à l'objet de la fondation, aux dépenses, à la nomination et aux devoirs des divers employés. Nous allons en rapporter textuellement les dispositions les plus essentielles.

“Attendu qu'on n'enseigne point ès écoles de „ médecine à faire les opérations de pharmacie... " le sieur Bouvard nous auroit supplié que trois " docteurs par lui choisis dans la faculté de Paris, " soient par nous pourvus pour faire aux écoliers "la démonstration de l'intérieur des plantes, et " de tous les médicamens, et pour travailler à la " composition de toute sorte de drogues, par voie "simple et chimique.... A ces causés nous avons " créé, à titre d'office, trois de nos conseillers" médecins de la faculté de Paris, qui auront la "qualité de démonstrateurs et opérateurs phar- 
PREMIÈ ÉPOQUE.

") maceutiques de notre Jardin, pour faire la dé-

"monstration de l'intéricur des plantes, et pour

" travailler à toutes les opérations pharmaceu"tiques nécessaires pour instruire les écoliers: "auxquels offices il sera par nous pourvu des " personnes de MM. Jacques Cousinot, Urbain

"Baudineau et Cureau de la Chambre.

"Voulons que dans un cabinet de ladite maison, "il soit gardé un échantillon de toutes les dro-

"gues, tant simples que composées, ensemble

"toutes les choses rares en la nature qui s'y ren-

" contreront; duquel cabinet ledit La Brosse aura

" la clef et régie, pour en faire l'ouverture aux " jours de démonstration.

"Et d'autant que ledit La Brosse, qui aura tout " le soin de la direction et culture du Jardin, ne " pourra toujours vaquer à faire la démonstration " extérieure des plantes, avons aussi créé à titre "d'office un sous-démonstrateur, pour l'aider à "faire la démonstration extérieure dans le Jar" din, duquel office, sera pourvu par nous Ves" pasien Robin, notre arboriste; chacun desquels „officiers vaquera à l'exercice de sa charge, aux " jours et heures qui lui seront désignés par notre ») surintendant.... à tous lesquels avons attribué "les gages qui suivent, savoir : à notre premier "médecin surintendant de toute l'curre, 3, oooliv., 》) à chacun des trois démonstrateurs, I,50o liv., à 
"La Brosse et à ses successeurs intendans, 6,00o " liv., au sous-démonstrateur, I,200 liv.

"Voulons aussi que ledit La Brosse dispose des " logemens, à la réserve de ce qui sera bâti après " pour l'instruction, le laboratoire et le cabinet " pour la conservation des échantillons et raretés, " il choisira les jardiniers, portiers, etc. : pour "l'entretien duquelJardin... nous avons ordonné à "l'intendant une somme de 4000 liv. par an outre "ses gages : .... donnons aux démonstrateurs et " opérateurs pharmaceutiques 400 liv. pour l'a" chat des drogues, et 400 liv. pour le salaire des " garçons servans au laboratoire. "

"Pour le payement desquelles sommes sera par " nous fait un fonds de 2 I, ooo livres, etc. Jonné " à Saint-Quentin, au mois de mai ı 635, registré "le I 5 mai."

Il est important de faire remarquer ici que la livre tournois valait, à cette époque, 2 fr. $50 \mathrm{c}$. de notre monnaie, ainsi les 21,000 liv. représentent maintenant 52,500 fr. L'augmentation de la dépense n'est nullement en rapport avec celle de l'établissement.

La faculté de médecine forma opposition à l'enregistrement de l'édit: elle demandait que les professeurs fussent choisis sur sa présentation et non sur celle du surintendant, elle se plaignait du choix de La Brosse : elle voulait surtout que la 
chimie ne fùt point enseignée : cette opposition n'eut pas de suites.

Guy de La Brosse s'établit dans le local dont on avait fait l'acquisition; il fit réparer et disposer les bâtimens, et il dressa, dès la première année, un parterre de 45 toises de longueur sur 35 de largeur, où il plaça toutes les plantes qu'il put se procurer, et dont la plupart lui furent données par Jean Robin, père de Vespasien, qui depuis plusieurs années les cultivait chez lui, et avait le titre d'arboriste ou simpliciste du roi. Le nombre de ces plantes; en y comprenant les variétés, était, en I 636 , de 1800 . Il fit ensuite défoncer les terrains; il se procura de nouvelles plantes par ses correspondances; il traça le plan du jardin dans une étendue de ro arpens, et il en fit l'ouverture en 1640 (r). En 164r, il publia le catalogue des plantes qui y étaient cultivées, dont le nombre, y compris les variétés, était de 2360. Il fit dessiner celles qu'il craignait de perdre, et en fit même graver quelques-unes.

Malheureusement il termina sa carrière lorsque ayant surmonté les obstacles qui lui avaient été opposés, il commençait à voir le succès de ses travaux. C'est vraiment lui qui est le fondateur du

(1) On écrivit sur la porte principale Jardin royal des Herbes médicinales, et cette inscription y est restée jusquaau moment où Buffon a agrandi le batiment. 
Jardin du Roi : il y mourut en $\mathrm{r} 643$; ses restes furent déposés dans la chapelle qui faisait partie du bâtiment; et lorsqu'on a été obligé de démolir cette chapelle pour construire l'escalier des galeries, ils ont été placés dans un caveau particulier.

Telle est l'origine d'un établissement, qui de nos jours est parvenu à un si haut degré de prospérité. Les bornes de cette notice nous obligent à ne tracer que très-rapidement l'histoire de ses progrès et de ses vicissitudes, jusqu'à la fin du dernier siècle. Nous nous arrêterons davantage à faire connaître les changemens opérés par la nouvelle organisation, pour donner une idée exacte de son état actuel.

La mort de Guy de La Brosse fut un grand malheur pour le Jardin du Roi ; ceux qui lui succédèrent n'eurent ni le même zèle ni la même activité. Les professeurs, révocables à volonté par le surintendant, changèrent plusieurs fois, et les lecons ne furent pas faites avec exactitude. Bouvard de Fourqueux, fils du premier médecin et conseiller au parlement, fut nommé à la place de Guy de La Brosse; il était impossible qu'il donnât ses soins à la culture et à l'enseignement, et Vespasien Robin fit seul les leçons de botanique. Cependant le surintendant obtint des fonds pour la construction d'une serre et pour celle du grand bassin qui est en face du cabinet. 
Bouvard ayant quitté sa place de premier médecin du roi, voulut conserver celle de surintendant. Elle lui fut disputée par Vautier, qui l'emporta, parce que d'après les termes de l'édit, l'intendance du Jardin était annexée à la charge de premier médecin. Celui-ci, qui était ennemi de Fourqueux, voulut nommer un intendant à son choix, et comme il éprouva des oppositions, il ne prit plus aucun intérêt au Jardin. Dès lors tout tomba en décadence; les plantes périrent faute de culture, et les leçons furent négligées. Cependant Vautier rendit un véritable service, en substituant un cours d'anatomie à celui qui était désigné sous le nom de l'intérieur des plantes, et qui était destiné à faire connaître les propriétés qu'on leur supposait.

La place deVautier, vacante par sa mort en I 652, fut donnée à Vallot. Quelques années avant cette époque, Gaston d'Orléans, frère de Louis XIII, avait établi dans son palais de Blois un jardin de botanique qui avait acquis beaucoup de célébrité par les ouvrages de Morison. Cette circonstance réveilla l'attention de Vallot; il nomma pour successeur de Robin, Denis Jonquet, médecin qui cultivait des plantes à Saint-Germain-des-Prés. Celui-ci fut secondé par Fagon, petit-neveu de Guy de La Brosse, qui ayant été élevé dans le Jardin, y avait puisé le goût de la botanique, et qui 
affectionnait beaucoup le lieu où il avait passé son enfance. Ce jeune homme, devenu célèbre depuis, voyagea à ses frais dans plusieurs provinces de France, dans les Alpes et les Pyrénées, et fit passer au Jardin tout ce qu'il put recueillir.

On fit, en même temps, venir des plantes des pays étrangers; et dès r 665 , le nombre des espèces cultivées, en y comprenant les variétés, se trouva de 4,0oo. Pour récompenser le zèle de Fagon, Vallot le nomma d'abord professeur de chimie, et ensuite professeur de botanique, à la mort de Jonquet, en ז67ז. Ainsi Fagon réunit les deux chaires de botanique et de chimie.

Gaston d'Orléans ne s'était point contenté de rassembler dans son Jardin de Blois, des plantes de tous les pays, et de confier à de savans botanistes le soin de les décrire, il avait voulu faire peindre sur vélin les espèces les plus remarquables, et il avait chargé de ce travail le peintre Robert, l'homme de son temps le plus habile en ce genre. Après la mort de Gaston, en i66o, Colbert engagea le roi à acquérir ces peintures, et à attacher à son cabinet un peintre qui serait obligé d'en ajouter chaque année un certain nombre. Robert fut nommé à cette place, et les ouvrages qu'il fit jusqu'à sa mort, arrivée en i684, sont d'une vérité et d'un fini, qu'aucun de ses successeurs n'a pu surpasser. 
Le même titre fut après lui donné à J. Joubert, peintre de paysage. Celui-ci n'avait pas le genre de talent qui convient à l'histoire naturelle, mais heureusement il se fit aider par Aubriet, dessinateur très-habile, qui fut d'abord attaché au Jardin, et ensuite nommé son successeur.

C'est ainsi que s'est formée la magnifique collection de figures de plantes et d'animaux, qui fut d'abord déposée à la Bibliothèque du Roi, et qui est aujourd'hui la principale richesse de celle du Muséum.

Vallot étant mort en I67ı, Colbert réunit la surintendance du Jardin à celle des bâtimens du Roi, dont il était pourvu; laissant au premier médecin le titre seul d'intendant, avec la direction des cultures. Il fit rendre, au mois de décembre, une déclaration du roi, qui réglait l'administration du Jardin, et il fit donner aux professeurs des brevets qui rendaient leurs places fixes. C'est de ce moment que l'établissement commença à prendre quelque importance : il eût pu s'accroître bien plus rapidement, si l'administrateur principal n'eût pas eu d'autre occupation.

D'Aquin, premier médecin du roi, et intendant du Jardin en 1672 , ne favorisa que l'anatomie. Cette science fut enseignée avec le plus grand succès, surtout par le célèbre Guichard-Jossph Du- 
verney, nommé professeur en 1679 , dont les leçons attirèrent un grand nombre d'élèves.

Fagon, qui avait rempli pendant plusieurs années les chaires de botanique et de chimie avec la plus grande supériorité, se trouvant chargé d'autres fonctions, crut devoir se faire remplacer, et désirant se nommer un successeur digne de lui, il fit venir du fond de la Provence Joseph Pitton de Tournefort, qui, n'ayant encore que vingt-six ans, annonçait déjà ce qu'il devait être un jour, et il lui transmit sa chaire de botanique en I683. Dix ans après il fut nommé premier médecin : cette place lui donna l'intendance du Jardin; et la considération dont il jouissait, fit rétablir en sa faveur le titre de surintendant.

Dès lors il lui fut facile d'obtenir les faveurs du gouvernement, pour l'établissement qu'il affectionnait, et pendant quinze ans qu'il en eut la direction tout reprit une nouvelle vie. Cette prospérité s'accroissant sans cesse serait parvenue au plus haut degré, si Fagon, réfléchissant qu'elle était due à son zèle et à ses lumières, eût craint que ses successeurs n'eussent pas les mêmes vues que lui, et s'il eût profité de son crédit pour faire donner au Jardin du Roi une administration stable, indépendante des caprices d'un chef.

Nous avons dit que Duverney occupait la chaire d'anatomic, et Tournefort celle de botanique. Ces 
deux sciences furent enseignées avec le plus grand éclat, et le nom des deux professeurs répandit du lustre sur l'établissement auquel ils étaient atlachés. La réputation que Tournefort s'était promptement acquise par ses leçons, s'accrut encore en I693, par la publication de ses Élémens de bolanique, qui, en présentant une méthode nouvelle pour la classification des plantes, donnaient à cette science une base solide, et en rendaient l'étude aussi facile qu'agréable.

Tournefort fit plusieurs royages pour se procurer des plantes, et en 1700 il alla au Levant accompagné du peintre Aubriet, attaché au Jardin, et qui devait dessiner les espèces nouvelles. Pendant son absence, la chaire fut remplie par son confrère Morin. A son retour en I 702 , il introduisit au Jardin beaucoup de plantes inconnues jusqu'alors, et qui sont aujourd'hui généralement cultivées chez les amateurs.

Tournefort mourut en 1708 , et laissa au Jardin. sa collection d'histoire naturelle et son herbier. Cet herbier n'est pas nombreux en espèces, mais il est précieux, en ce qu'il renferme les plantes recueillies au Levant, et indiquées dans le Corollaire des Institutiones rei herbarice (I).

(1) Les Institutiones rei herbaria sont une traduction angmentee des Élémens de botanique, Tournefort les publia en $x$ 700; toutes les plantes y sorat indiquées par une phrase. Le corollarium y fut ajouté après le retour du voyage au Levant. 
Danty d'Isnard fut nommé à la place de professeur de botanique; mais sa santé ne lui permit pas de la conserver long-temps. L'académie des sciences l'admit en I 7 I 6 .

Fagon, que ses fonctions de premier médecin retenaient à la cour, aurait pu, comme ses prédécesseurs, placer au Jardin un intendant auquel il aurait transmis son autorité ; mais il jugea qu'il valait beaucoup mieux confier tout simplement la direction des cultures à un homme instruit, et que rien ne détournerait des soins dont il serait chargé : il fit choix de Sébastien Vaillant, qui, après avoir entendu les leçons de Tournefort, en $\mathbf{6 g} \mathrm{I}$, avait renoncé à sa place de chirurgien à l'Hôtel-Dieu, pour s'occuper uniquement de l'étude des plantes. Ce jeune homme surpassa les espérances qu'on avait concues de lui, et s'acquit bientôt une réputation qui le fit entrer à l'académie des sciences. En 1708 , Fagon le nomma sousdémonstrateur pour suppléer le professeur en cas d'absence, et pour conduire les élèves à la campagne et leur faire connaître les plantes indigènes. Il le chargea aussi d'acquérir des objets nouveaux pour le droguier, qui fut confié à sa garde et disposé pour l'instruction, et il lui fit accorder des fonds pour la construction de deux serres chaudes.

Vaillant fit un herbier très-considérable et très- 
bien soigné : chaque espèce était rapportée à son genre, et accompagnée d'une étiquette, qui contenait les différens noms par lesquels la plante avait été désignée, et l'indication du lieu où l'échantillon avait été cueilli. Cet herbier dont le roi fit l'acquisition lorsque Vaillant mourut en I 722 , a été disposé dans un ordre méthodique, et fait encore aujourd'hui la base de l'herbier du Muséum.

Ce qui doit surtout immortaliser le nom de Vaillant, c'est le discours d'ouverture qu'il prononça en I 7 I 6 , lorsqu'il fut chargé de suppléer le professeur de botanique alors absent. Dans ce discours il démontre l'existence des deux sexes et le phénomène de la fécondation dans les régétaux. Ainsi c'est au Jardin du Roi que cette grande découverte qu'on avait seulement aperçue, et qui n'était point généralement adoptée, fut pour la première fois annoncée d'une manière positive, et appuyée de preuves incontestables.

Danty d'Isnard s'étant démis de sa place de professeur, après avoir fait un seul cours, Fagon, qui pendant 25 ans s'était applaudi du choix qu'il avait fait de Tournefort, cherchait à découvrir parmi les élèves de ce grand maître, celui qui serait le plus capable d'enseigner sa doctrine, et d'occuper dignement la chaire qu'il avait illustrée. Un jeune homme de Lyon, qui après avoir étudié la médecine à Montpellier, était venu à 
Paris, pour se perfectionner dans l'étude des plantes sous le professeur dont il avait admiré les ouvrages, lui parut à tous égards propre à remplir ses vues; c'était Antoine de Jussieu, dont le nom est devenu si célèbre par les progrès que lui-même, ses deux frères et son neveu ont fait faire à la botanique. Fagon ayant eu plusieurs occasions de s'entretenir avec lui et d'apprécier son mérite; le choisit parmi tous les concurrens qui se présentaient, et le nomma à la place de professeur, en 1709, quoiqu'il ne fût âgé que de 23 ans. Antoine de Jussicu justifia bientôt ce choix, par les talens qu’il montra dans ses leçons, et par plusieurs mémoires qui, en 1712 , le firent admettre à l'académie des sciences. En 1716, il alla parcourir l'Espagne et le Portugal, et l'année suivante, il en rapporta un grand nombre de plantes qui cnrichirent le Jardin. Son frère Bernard, âgé de 17 ans, l'accompagna dans ce voyage, et prit dès lors la résolution de renoncer à la médecine, pour se livrer uniquement à la botanique, à laquelie il imprima dans la suite une nouvelle direction. Ce fut Antoine de Jussieu qui en 1720 remit au chevalier Declieux, enseigne de vaisseau, un pied de café qui, transporté à la Martinique, y a produit tous ceux qu'on cultive aux Antilles.

Fagon qui avait quitté sa chaire de botanique en I683 s'étail fait remplacer pendant plusieurs 
années dans celle de chimie par des professeurs habiles tels que Saint-Yon, Louis Lemery, Berger et Geoffroy : mais ce dernier s'étant fait une grande réputation par ses leçons, Fagon pensa qu'il ne saurait avoir un plus digne successeur, et il lui céda entièrement sa place en I 7 I2.

Geoffroy enseigna avec le plus grand succès la chimie et la matière médicale; il était aidé par des démonstrateurs qui faisaient les expériences chimiques et pharmaceutiques, et ces démonstrateurs furent presque tous des savans distingués. Ce ne fut cependant qu'en 1695 que cette place de démonstrateur fut donnée à un professeur titulaire ; jusqu'alors elle avait été remplie par commission.

Depuis 1679 que Duverney fut nommé à la place de professeur d'anatomie, cette science continua d'être enseignée au Jardin, bien mieux qu'elle ne l'était partout ailleurs en France; et les progrès qu'elle a faits sous les successeurs de ce grand maître, sont la suite de la méthode qu'il avait introduite, et de l'enthousiasme qu'il avait excité par ses leçons.

Fagon jouissait du fruit de ses travaux. Les trois chaires étaient occupées par des hommes célèbres, et les démonstrateurs qui les secondaient, étaient eux-mêmes des savans du plus grand mérite. On avait construit, à gauche de la porte d'entrée, un 
amphithéâtre qui pouvait contenir six cents personnes, et qui était presque toujours rempli : la culture était dirigée arec soin par Vaillant : on se procurait des plantes par des correspondances en Amérique : le droguier commençait à renfermer des choses intéressantes : plusicurs pièces d'ostéologie avaient été jointes à la collection : Aubriet, qui, depuis près de quinze ans, avait succédé à Joubert dans la place de peintre du cabinet, continuait à dessiner des plantes et des animaux.

Telle était la situation de l'établissement à la mort de Louis XIV en 1715. Fagon, âgé et infirme, se démit de sa place de premier médecin, qui fut donnéc à Poirier, et il se retira au Jardin où il avait pris naissance, et où il mourut en I 7 I 8 .

Sa mémoire, dit M. de Jussieu, sera toujours en vénération dans l'établissement, auquel il procura de nombreuses collections, des locaux propres à les recevoir et à les conserver, et surtout des professeurs qui honorèrent leurs places.

Poirier, surintendant à la mort de Fagon, ne lui survécut que de quelques jours; Dodart lui succéda dans la place de premier médecin; mais l'administration du Jardin, détachée de cette place par une déclaration du roi de 1718 , fut donnée à Chirac, médecin du duc d'Orléans, avec le titre d'intendant.

Chirac, uniquement occupé de la pratique 
de la médecine, ne prenait aucun intérêt aux sciences naturelles; il voulait cependant que rien ne fût fait que par ses ordres, et que les professeurs fussent sous sa dépendance et soumis à un règlement qu'il était impossible d'exécuter. Voyant enfin qu'il ne pouvait tout gouverner par lui-même, il plaça au Jardin, avec le titre d'inspecteur, un homme sans instruction, et qu'un ordre supérieur le força de renvoyer. Les correspondances furent entravées, les cultures négligées, et les fonds destinés pour l'établissement détournés pour đ'autres objets, sans qu'il en fût rendu aucun compte. Son crédit étant encore augmenté lorsque, par la mort de Dodart, il devint premier médecin, les professeurs ne purent faire entendre leurs réclamations, et le Jardin tomba en décadence, malgré les soins que se donnaient Antoine et Bernard de Jussieu, et les sacrifices qu'ils ne cessaient de faire pour se procurer et les engrais et les instrumens de culture et tous les secours qu'on leur refusait : leur zèle déplaisait même à Chirac. Bernard de Jussieu qui, en I 722, avait succédé à Vaillant dans la place de sous-démonstrateur, et qui, en cette qualité, faisait les herborisations à la campagne et surveillait les cultures, était aussi chargé du soin du droguier, auquel on commençait à donner le nom de Cabinet d'histoire naturelle : Chirac lui ôta cette place. 
Aubriet contimuait à travailler pour la collection des vélins ; mais l'intendant lui demandait de dessiner de préférence lesplantes officinales qui sont généralement connues.

L'enseignement de l'anatomie et celui de la chimie éprouvèrent moins d'obstacles, soit parce que tout se bornait à des leçons et n'exigeait point de frais, soit parce que Chiracfaisait plus de cas de ces sciences que des autres parties de l'histoire naturelle. Duverney choisit pour faire ses démonstrations des collaborateurs très-habiles, parmi lesquels it faut compter son frère Pierre Duverney, et son neveu Jacques-François-Marie Duverney pour qui l'on créa dans la suite la place de démonstrateur, et qui fut le maître de Daubenton. Pendant les dernières années de sa vie, se trouvant trop affaibli pour continuer son cours, il se fit suppléer par Winslow son élève, qui avait déjà beaucoup de réputation. Il mourut en $\mathbf{7} 30$, dans la quatrevingt-deuxième année de son àge et la cinquanteunième de son professorat. I La voix publique appelait Winslow à exercer comme titulaire les fonctions dans lesquelles il s'élait distingué comme suppléant : des circonstances particulières lui firent préférer Hunaud son élève. Heureusement cet élève, déjà membre de l'académie des sciences, joignait à beaucoup de savoir une grande facilité d'élocution; il soutint la réputation de la 
PREMIÈRE ÉPOQUE.

chaire d'anatomie du Jardin, et fut constamment suivi par un grand nombre d'élèves.

Greoffroy qui enseignait la chimie depuis I 7 10, étant mort en I $^{3}$ I , sa chaire fut donnée à Louis Lemery. Ce nouveau professeur répandit sur la science une clarté qu'elle n'avait pas eue encore, et l'étendit au delà de la matière médicale à laquelle on la réduisait dans les cours.

Le professeur de chimie s'adjoignit constamment un collaborateur chargé de faire les expériences et les préparations pharmaceutiques. II est à remarquer que ceux qui remplirent cette fonction, soit pour l'anatomie, soit pour la chimie, furent presque tous membres de l'académie dessciences. Simon Boulduc, savant très-distingué, fut le premier qui eut le titre de démonstrateur. A sa mort, en 1729 , sa chaire fut donnée à son fils Gilles-François Boulduc.

Chirac étant mort en I 732 , son gendre, Chicoisneau lui succéda dans la place de premier médecin; mais celle d'administrateur du Jardin du Roi en fut pour toujours séparée. On reconnut alors la nécessité de la confier à un homme qui pût s'en occuper exclusivement. Le roi y nomma, avec le titre d'intendant, Charles-Francois de Cysternay du Fay : et ce choix est la première cause des progrès que l'établissement n’a cessé de faire jusqu'à nos jours. 
Du Fay était d'une ancienne famille qui avait fai profession des armes depuis le $\mathbf{X V}^{\mathrm{e}}$ siècle. Son père et son aieul aimaient les sciences, et ils en avaient inspiré le goût à leur fils. Le jeune du Fay avait comme eux embrassé l'état militaire, et s'était distingué dans la guerre d'Espagne; mais ses momens de loisir étaient employés à l'étude de l'histoire naturelle, de la physique et de la chimie, et il les cultiva avec tant de succès, qu'il fut reçu membre de l'académie des sciences en I 23. A la paix il quitta le service, et sa nomination à la place d'intendant du Jardin mit le comble à ses voux. Il avait alors trente-cinq ans, et il résolut de consacrer sa vie entière à relever et à faire fleurir l'établissement dont il était le chef. Il s'occupa d'abord à réparer les désordres produits par l'administration antérieure; il porta sa principale attention sur la botanique, et rendit à Bernard de Jussieu la place de garde du cabinet d'histoire naturelle; il obtint du ministre les fonds nécessaires pour réparer ce qui était tombé en ruine; il fit des voyages en Angleterre et en Hollande pour établir des correspondances et pour enrichir l'établissement; il rendit stable la place de démonstrateur d'anatomie en y faisant nommer, comme titulaire, vers I 736 , J.-F.-M. Duverney, neveu du professeur; it fit transporter au cabinet des objets rares et utiles 
pour l'instruction, et il lui donna sa collection de pierres précieuses. En I 739 , il fut atteint de la petitc-vérole; il jugea qu'il ne survivrait point à cette maladie; et voulant assurer la prospérité de l'établissement, et mettre le comble aux services qu'il avait rendus, il écrivit au ministre pour demander que Buffon füt son successeur. 


\section{DEUXIÈME ÉPOQUE.}

DEPUIS L'ENTRÉE DE BUFFON AU JARDIN DU ROI EN $17 \bar{J} 9$, JUSQU'A SA MORT EN 1788.

Buffon était connu par plusieurs Mémoires de mathématiques, de physique et d'économie rurale, qui lui avaient ouvert l'entrée de l'académie des sciences; mais il ne l'était pas comme naturaliste. Doué de cette capacité d'attention qui fait découvrir les rapports les plus éloignés, et de cette imagination brillante qui appelle l'attention desautres sur lesrésultats auxquels onn'estsoi-même parvenu qu'à force de travail, il pouvait se distinguer également dans tous les genres, maisilnes'étaitpas encore décidé sur le choix de la science à laquelle il appliquerait la force de son esprit et les connaissances exactes qu'il avait acquises. Sa nomination à la place d'intendant du Jardin du Roi, le détermina à s'attacher à l'histoire naturelle; et comme les progrès de cette science tenaient à la prospérité de l'établissement qui leur était consacré, il mit sa gloire à le rendre en tout digne de sa destination. A mesure que sa réputation s'étendit, il 


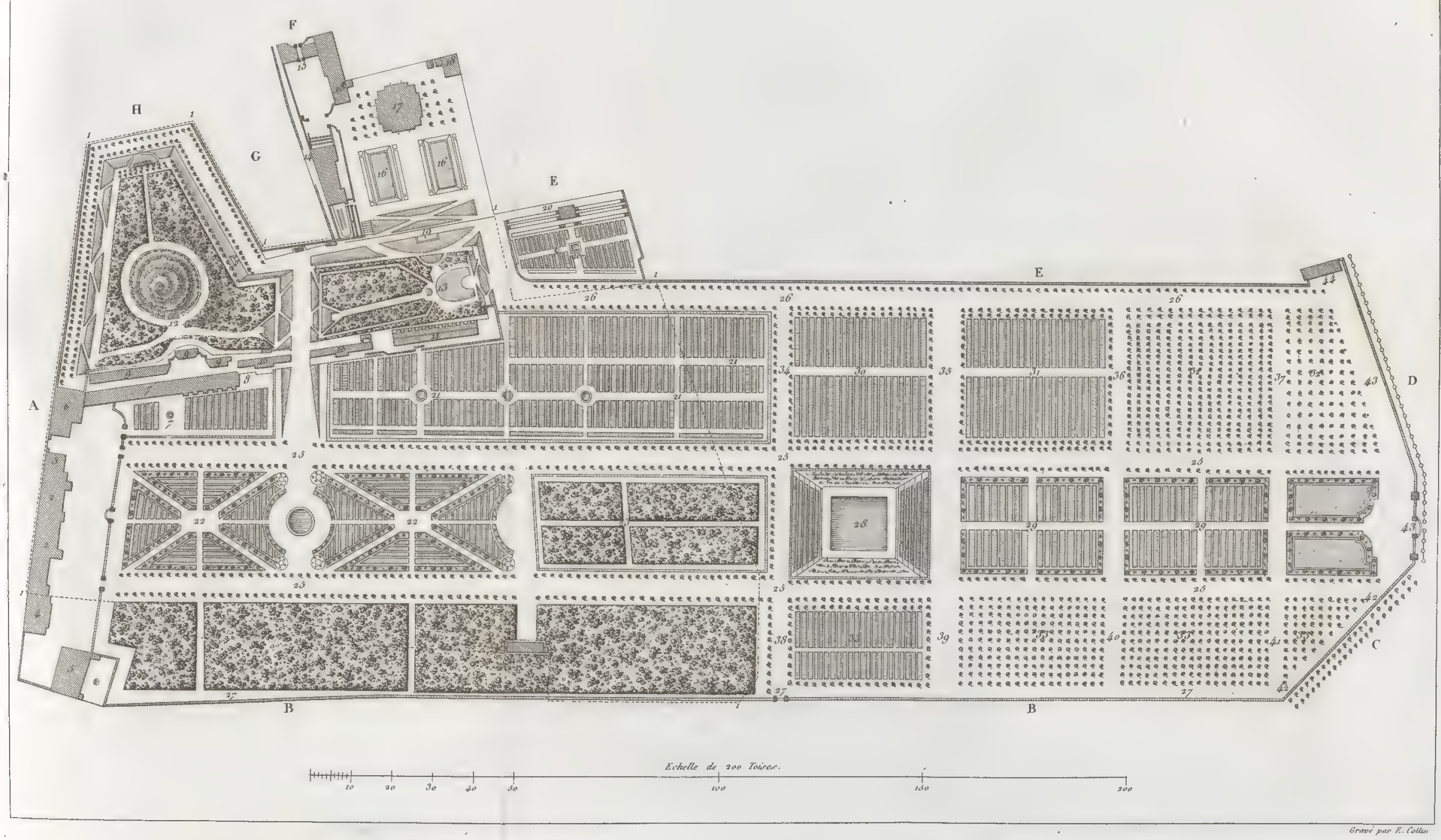




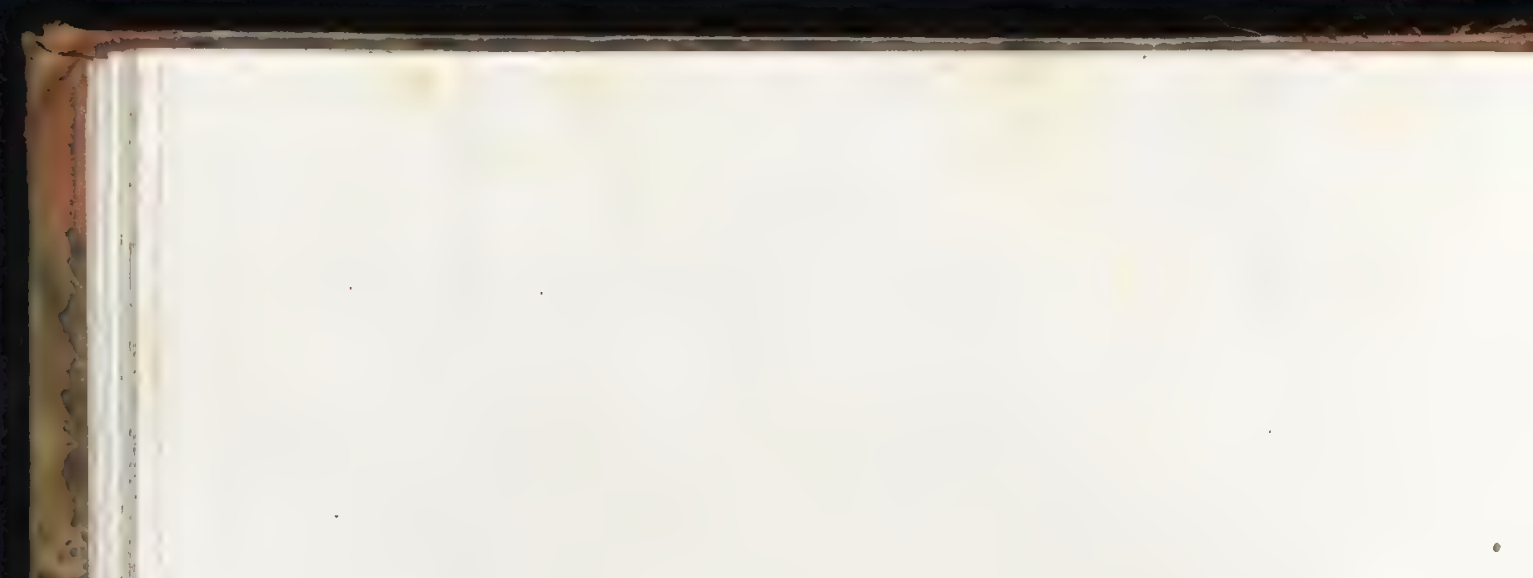


profita des avantages que lui donnaient son crédit et sa célébrité pour enrichir le monument auquel il avait lié son existence. C'est à lui que le Jardin du Roi doit ses accroissemens jusqu'à la nouvelle organisation, et c'est à cause de l'étendue qu'il lui avait donnée et de la variété des objets qu'il y avait réunis, que cette nouvelle organisation est devenue nécessaire.

Lorsqu'on veut construire un vaste palais, un seul architecte doit en concevoir l'ensemble, en ordonner la distribution, en tracer le plan, en diriger les premiers travaux. Mais une fois que les fondemens sont jetés, il faut que d'habiles artistes soient chargés chacun d'une partie, qu'ils s'attachentà en perfectionner les détails, et qu'ils se concertent entre eux pour mettre de l'accord dans leurs opérations. L'attention du même individu ne saurait se porter à la fois sur plusieurs objets, et des choses essentielles seraient négligées pour d'autres qui le seraient moins.

Sile Jardin etle cabinet du Roi doivent à Buffon la splendeur à laquelle ils sont parvenus, c'est à l'existence de ce magnifique établissement que Buffon doit toute sa gloire. S'il n'eût été placé au centre des collections, si le gouvernement ne lui eût fourni le moyen de les augmenter, s'il n'eût pas été en relation avec tous les naturalistes, il n'aurait jamais conçu le plan de son Histoire naturelle, et 
l'eût-il conçu, il n'aurait pu l'exécuter. Le génie, qui peut seul embrasser à la fois un grand nombre de faits pour en tirer des conclusions générales, serait continuellement exposéà l'erreur s'il n'avait à sa portée tous les élémens de ses spéculations.

Lorsque Buffon entra au Jardin du Roi, le cabinet consistait en deux petites salles, une autre pièce renfermait des squelettes qu'on ne montrait point au public; les herbiers étaient dans l'appartement du démonstrateur de botanique : le jardin borné à la hauteur de la pépinière actuelle du côté du levant, à cclle des serres du côté du nord, à celle des galcries d'histoire naturelle du côté du couchant, offrait encore des terrains vagues, et l'on n'y royait ni allées, ni plantations régulières.

Buffon donna ses premiers soins à l'augmentation du cabinet, à l'agrandissement du local destiné à renfermer les collections. Elles furent disposées dans deux grandes salles du bâtiment des galeries actuelles, qui était auparavant le logement de l'intendant, et bientôt l'entrée en fut ouverte au public à des jours déterminés.

Il s'occupa ensuite de l'embellissement du jardin. Après avoir fait abattre une allée ancienne, tracée dans la longueur du terrain des cultures, et qui ne répondait pas à la porte principale, il la remplaça, en 1740 , par une allée de tilleuls, dans la direction convenable, et il fit planter une allée 
parallèle de l'autre côté du parterre. Ces allées, qui existent depuis quatre--vingts ans, se terminent vel's l'extrémité de la pépinière, et marquent les limites du jardin à cette époque. Elles ont été prolongées ensuite lorsqu'on a acquis de nouveaux terrains.

Depuis que la garde du cabinet avaitété rendue à Bernard de Jussieu, il n'avait pas discontinué de donner ses soins à l'arrangement et à la conservation des objets. L'étendue de ses connaissances, etla faculté qu'il avait de saisir les rapports et de classer les êtres dans l'ordre le plus naturel, le rendaient fort propre à remplir cette tâche devenue plus difficile par l'accroissement de la collection; mais détourné par d'autres travaux, et ne demeurant point au Jardin, il témoigna le désir d'être remplacé dans une fonction qui exigeait beaucoup d'activité et une assiduité de tous les momens. Buffon sentait de son côté qu'il avait besoin d'être aidé dans ses recherches d'histoire naturelle par un homme qui eût encore toute la vigueur de la jeunesse, et qui possédât au plus haut degré l'esprit de méthode et le talent de l'observation. Doué de cette force de génie qui saisit les traits principaux des objets et les reproduit dans de magnifiques tableaux, il n'avait ni le temps ni la patience nécessaires pour examiner les détails, et sa vue, assez faible, ne le luí permettait même pas. 
Il jeta les yeux sur son compatriote Daubenton qui avait alors vingt-neuf ans, et qui, après aroir étudié la botanique sous les Jussieu, l'anatomie sous Winslow et Durerney, s'était retiréà Montbard, lieu de sa naissance, pour y exercer la médecine.

Il le fit venir à Paris, et lui procura, en 1745 , la place de garde et de démonstrateur du cabinet, avec un logement au Jardin et des appointemens qui, de 500 francs, furent bientôt portés à 4,000. Il le chargea de l'arrangement du cabinet, et se l'associa pour la partie descriptive de son Histoire naturelle, et surtout pour l'anatomie.

En publiant, en I 749 , les premiers volumes de son Histoire naturelle qui attirèrent l'attention de l'Europe, Buffon fit un appelà tous les naturalistes qu'il invitait à lui envoyer ce qu'ils auraient trouvé de plus remarquable. Il déposait au cabinet ce qu'il recevait; Daubenton le mettait, en ordre; et bientôt le local devint trop resserré pour contenir tant de richesses. Buffon, quiavait déjà sacrifié une partie de son logement, crut devoir l'abandonner en entier, et, en 1766 , il transporta son domicile rue des Fossés-Saint-Victor, $n^{\circ}$ I 3 . Alors la collection fut disposée dans quatré grandes salles, qui ont formé seules le cabinet jusqu'à la nouvelle organisation. Les deux premières salles furent destinées aux animaux; la troisième aux 
minéraux; la quatrième aux herbiers, à l'ancien droguier et aux divers produits du règne végétal (I).

Ces salles furent ouvertes au public deux jours de la semaine, et les élèves eurent des heures réservées pour l'étude. Daubenton, présent aux séances, donnait les éclaircissemens qui lui étaient demandés, et souvent des naturalistes étrangers venaient le trouver pour s'instruire auprès de lui.

Il partageait ainsi son temps entre le soin minutieux de nommer et d'arranger les objets, et celui de diriger les jeunes gens qui se livraient à l'étude. Sa patience ne se lassait point ; mais bientôt le travail dont il s'était chargé devint si considérable, qu'il lui fut impossible d'y suffire. Buffon, qui reconnut la nécessité de lui donner un adjoint, obtint du ministre la création d'une place de sousdémonstrateur, et il y fit nommer Daubenton le jeune, cousin du précédent, auquel on assura un traitement de 2,40o livres, avec un logement audessus des salles du cabinet.

Tandis que les collections s'accroissaient d'année en année, soit par les envois des naturalistes et des voyageurs, soit par les acquisitions que le roi faisait à la sollicitation de Buffon, l'enseigne-

(1) Les squelettes continuèrent de rester dans un appartement isolé : comme l'anatomie comparée n'occupait alors qu'un très-petit nombre de savans, on ne mettait point en évidence des objets qui ne paraíssaient pas propres à exciter la curiosité. 
IISTUIRE DU MUSÉUM.

ment était suivi avec beaucoup d'activité; non que Buffon attachàt la mème importance à toutes les parties, mais parce qu'elles étaient professées par des hommes habiles, et qui savaient faire eux-mêmes des sacrifices pour la prospérité de l'établissement.

Antoine de Jussieu, qui professait la botanique depuis 1710 , ne se rendait pas seulement utile par ses leçons. Il envoyait à ses frais des jeunes gens parcourir les provinces pour y recueillir des graines et des plantes dont il enrichissait le Jardin. Il se composa une bibliothèque d'histoire naturelle, et il forma un herbier considérable, qui ont facilitéà son frère et à son neveu les moyens de se distinguer dans la botanique, et qui ont toujours été au service de ceux qui cultivent cette science, comme s'ils avaient appartenu au gouvernement; avec cet avantage que les possesseurs ne leur ont jamais refusé des instructions et des explications.

En 1758 , la chaire vacante par la mort d'Antoine de Jussieu, fut donnée à Louis-Guillaume Lemonnier, membre de l'académie des sciences, et médecin en chef de l'armée qu'on avait envoyée en Allemagne. Comme Bernard de Jussieu son ancien maitre, Lemonnier aimait la science pour elle-même, et il en inspirait le goût à ceux qui s'entretenaient avec lui. En 1770, il fut nommé premier médecin ordinaire du roi, et cette charge l'obligeant de résider à Versailles, il se fit 
DEUXIEME ÉPOQUE.

suppléer au Jardin par M. Antoine-Laurent de Jussieu, le professeur actuel, neveu de Bernard. Mais pendant toute sa vie il employa ses momens de loisir à cultiver des plantes et des arbres, qu'il faisait venir à ses frais des pays étrangers pour les propager dans sa patrie. Lorsque dans un âge avancé, la révolution lui enleva ses places et sa fortune, il trouva dans son occupation favorite une source inépuisable de consolations et de jouissances.

Bernard de Jussieu avait d'ahord dirigé seul toutes les cultures; mais le nombre des plantes qui exigent des soins particuliers, augmentant tous les jours, il forma un jardinier, nommé Bertamboise, qui mérita bientôt toute sa confiance. Celui-ci étant mort en $\mathbf{7}$ 45, Buffon le remplaça par Jean-André Thouin, jardinier à Stord, près l'IleAdam, qui se distingua dans cet emploi par son zèle et ses connaissances, et qui fut le chef de la famille devenue depuis si recommandable par les services qu'elle a rendus à tous les établissemens consacrés à la culture des végétaux, et particulièrement au Jardin du Roi.

Le jardinier en chef Thouin étant mort en 1768 , Bernard de Jussieu ne crut mieux pouvoir réparer cette perte qu'en faisant nommer à sa place son fils André Thouin, alors âgé de vingt ans. Ce jeune homme, élevé dans le Jardin, au milieu des végé- 
taux de tous les pays, instruit par les leçons des grands maîtres et passionné pour l'étude, acquit bientôtles connaissances étendues et positives qui l'ont fait entrer à l'ac'adémie des sciences, et l'ont fait nommer ensuite à la place de professeur de culture qu'il occupe aujourd'hui. Ce fut par les soins réunis du professeur, du démonstrateur et du jeune jardinier, que la collection de végétaux vivans devint en peu de temps plus nombreuse et plus intéressante qu'on n'avait oser l'espérer; car l'intendant, continuellement occupé du cabinet qu'il avait créé, donnait moins d'attention aux autres parties de l'établissement.

Nous verrons bientôt que les sollicitations de M. Antoine-Laurent de Jussieu le déterminèrent à porter ses vues sur la botanique, pour laquelle le Jardin avait d'abord été destiné, et que, lorsqu'en I $77^{2}$, il résolut de donner à l'établissement dont il était le chef toute l'étendue et toute la régularité possible, ce fut par l'école de botanique qu'il commença l'exécution de son plan: mais avant de parler de ce qui fut entrepris après $\mathbf{1} 772$, revenons sur l'enseignement de la chimie et de l'anatomie depuis ${ }^{7} 39$ jusqu'à cette époque.

Boulduc, démonstrateur de chimie, qui avait succédé à son père en 1729 , étant mort en $174 \%$, sa chaire fut donnée à Guillaume-François Rouelle, dont la réputation était déjà établie par des cours 
DEUXIEME ÉPOQUE.

particuliers et par des Mémoires qui, deux ans après, le firent entrer à l'académie des sciences. Rouelle avait au plus haut degré la passion de la chimie; son esprit, avide de décourertes, ne la considérait point comme l'art d'opérer des transformations singulières, ou de faire des préparations pharmaceutiques, mais comme un moyen de pénétrer les secrets de la nature et les lois de la composition des corps. Également ennemi des propriétés occultes, des explications vagues et du langage obscur des anciens chimistes, il était attaché à la doctrine de Stahl, qui, reposant sur des observations incomplètes, avait cependant l'avantage de coordonner les faits, et de ramener à un phénomène principal, tous les phénomènes secondaires. Cette théorie magnifique avait, depuis quelques années, donné une nouvelle direction à la chimie, mais elle n'était connue en France que de quelques savans, et elle avait encore de nombreux adversaires. Rouelle la professa de manière à la faire généralement connaître. Il appelait d'abord l'attention par des expériences surpre-nantes, et s'élevait ensuite à des vues générales. Son élocution était souvent incorrecte et familière, mais toujours animée et pittoresque, et l'enthousiasme dont il était transporté se communiquait à ses auditeurs. Ses leçons, en inspirant le goût de lá chimie, firent adopter les idées de Stahl, comme 
celles de Fourcroy répandirent ensuite les principes de Lavoisier et la nouvelle nomenclature. Lorsqu'en i 768 , le dérangement de sa santé l'obligea de discontinuer son cours, il se fit remplacer par son frère, qui devint titulaire en 1770 . Celui-ci n'avait pas de même le talent de frapper l'imagination de ses auditeurs; mais comme il connaissait à fond tous les procédés chimiques, il rendait ses leçons intéressantes par le choix des expériences.

Tandis que Rouelle répandait de nouvelles idées, Bourdelin continuait à enseigner la doctrine de Lemery, auquel il avait succédé en r743 : il en résulta la discordance la plus choquante entre les leçons du professeur et celles du démonstrateur, d'autant plus que celui-ci déclamait avec véhémence contre les opinions qu'il n'admettait pas. Mais Bourdelin, homme d'un esprit droit et d'un caractère paisible, et d'ailleurs entièrement livré à la pratique de la médecine, ne fut ni blessé des attaques de son collègue ni jaloux de ses succès. Il reconnaissait la supériorité de la nouvelle théorie, quoiqu'il n'eût plus le temps de l'étudier assez pour l'exposer lui-mêrne, et lorsque son grand àge l'empêcha de continuer ses leçons en $\mathbf{I} 77^{\circ}$, il se fit suppléer par Macquer, l'élève de Rouelle, dont nous aurons bientôt occasion de parler. 
DEUXIEME ÉPOQUE.

Pendant les premières années de son séjour au Jardin, Buffon eut le chagrin de perdre plusieurs des professeurs qu'il y avait trouvés. En reconnaissant les services qu'ils avaient rendus et qui ne doivent jamais être oubliés, il s'attacha à les remplacer par des hommes capables de donner un nouveau lustre à l'établissement. Hunaud ayant été enlevé aux sciences la même année que Boulduc, sa place fut donnée à Winslow, le plus célèbre anatomiste de l'Europe, comme celle de Boulduc l'avait été à Rouelle. Winslow, avait pendant long-temps, fait les leçons pour Duverney, dont il aurait dû être le successeur immédiat. Quoique âgé de soixante-quatorze ans, il reprit ses anciennes fonctions et les remplit avec le mème zèle que dans sa jeunesse. Il se trouvait heureux de se voir entouré de nombreux élèves qui l'écoutaient avec le respect dû à son âge et l'admiration qu'inspirait son génie. Mais après huit ans d'exercice il sentit le besoin du repos, et demanda un adjoint. Sa survivance fut donnée à Ferrein, déjà membre de l'académie et professeur au collége de France.

Ferrein, devenu titulaire à la mort de Winslow en 1760 , continua de remplir avec distinction la chaire d'anatomie. Sur la fin de sa vie, il se fit remplacer par M. Portal, alors fort jeune, mais déjà connu très-avantageusement par des 
cours particuliers. Il mourut en 1769 , et sa place fut donnée à Antoine Petit, qui avait une grande réputation comme médecin, comme anatomiste, et surlout comme professeur. Cette réputation s'accrut encore par la manière brillante dont il fit son cours au Jardin. Non-seulement les élèves en médecine, mais les gens du monde accouraient à ses leçons, et l'amphithéâtre ne pouvait contenir tous ceux qui s'y rendaient. Il savait répandre de l'intérêt sur les matières les plus arides, et ceux qui l'ont entendu il y a cinquante ans, conservent encore le souvenir de son éloquence.

L'établissement perdit en $\mathbf{7} 43$ le peintre Aubriet, qui avait accompagné Tournefort dans ses voyages et qui était le plus ancien des habitans du Jardin. Ses dessins sont très-nombreux dans la collection des vélins; ils n'ont pas le fini de ceux de Robert, mais ils sont d'une grande exactitude, et rendent parfaitement le port des plantes. Dans les dernières années de sa vie, il se fit remplacer par mademoiselle Basseporte, qui eut après lui le même titre et le même emploi.

Buffon était parvenu au plus haut degré de sa gloire; ses ouvrages, en le plaçant au premier rang parmi les écrivains de son siècle, avaient répandu partout le goût de l'histoire naturelle, et les collections qu'il avait formées facilitaient l'étude de cette science. Ii jouissait dans les pays étrangers 
de la plus grande considération, et tous ceux qui faisaient quelques découvertes ou quelques observations nouvelles, s'empressaient de les communiquer à l'homme de génie qui pouvait en les citant leur assurer une sorte d'immortalité.

Le moment était arrivé où il pouvait réaliser des projets qu'il avait long-temps médités pour en préparer plus sûrement l'exécution, lorsqu'en I77I, il fut atteint d'une maladie très-grave et qui causa les plus vives inquiétudes. Pendant sa convalescence, il apprit que M. le comte d'Angiviller avait obtenu du ministre la survivance de sa place d'intendant du Jardin; et il en fut extrêmement blessé. Mais M. d'Angiviller se conduisit de manière à regagner son amitié. Comme, en sa qualité de directeur des bâtimens du roi et de chef des académies de peinture et de sculpture, il était chargé de désigner aux artistes les grands hommes dont ils devaient exécuter les statues en marbre pour le compte du gouvernement, il demanda au roi la permission d'en ériger une à Buffon. C'était la distinction la plus flatteuse qu'il fût possible d'accorder à un homme vivant, puisqu'elle avait jusqu'alors été réservée à honorer après leur mort ceux qui avaient rendu les plus éminens services à leur patrie; mais le Roi, persuadé que le jugement unanime des contemporains était le présage de celui de la postérité, accueillit 
la proposition de M. d'Angiviller, et le célèbre Pajou fut chargé de l'exécution de la statue qui, terminée en $177^{6}$, fut placée dans l'escalier des salles du cabinet. On la voit aujourd'hui dans la bibliothèque du Muséum.

La santé de Buffon ayant été parfaitement

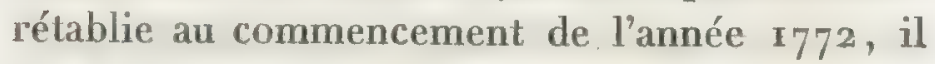
résolut de venir de noureau se loger au Jardin, et d'employer son crédit à donner à cet établissement l'étendue et le lustre dont il était susceptible.

En conséquence il fit acquérir par le gouvernement deux maisons voisines du Cabinet, qui furent réunies au Jardin, et dont on forma le logement nommé l'intendance, où il transporta son domicile. Le premier étage fut arrangé pour former son appartement, et les étages supérieurs furent destinés pour le dépôt des objets non encore placés dans les salles du Cabinet.

La rentrée de Buffon au Jardin est une époque très-remarquable. En effet, c'est de ce moment que tout prit un accroissement rapide, et que furent préparées les améliorations qui ont eu lieu depuis l'organisation nouvelle.

Pour faire connaitre les services que Buffon rendit à l'établissement pendant les seize ans qu'il l'eut encore sous sa direction, il faut les considérer sous trois points de vue: les constructions, les collections, et l'enseignement. 
DEU XIÈ ME ÉPOQUE.

L'école de botanique était encore la même que du temps de Tournefort. Les arbres étaient séparés des herbes, et plantés à une grande distance près de l'endroit où est maintenant un café. L'espace qui se terminait à l'extrémité des serres acluelles, était tellement insuffisant, qu'il fallait cultiver une partie des plantes, soit hors de l'école, soit dans les endroits où l'on trouvait une place vide, sans aucun égard à leur classification ou à leurs rapports naturels, et que le professeur était souvent obligé d'aller les démontrer dans une autre partie du Jardin. Le terrain était d'ailleurs épuisé, et les plantes délicates ne pouvaient s'y conserver qu'à force de soins. Buffon cédant aux sollicitations réitérées de M. de Jussieu, exposa au ministre, le duc de la Vrillière, les besoins du Jardin, et il en obtint en $177^{3}$ une somme de 36,000 liv. qui fut destinée au renouvellement de l'école de botanique.

On traça des plates-bandes, on fit défoncer les terrains, et les plantes levées en automme avec les précautions convenables, furent, à la fin de l'hiver, transplantées dans le lieu qu'elles devaient occuper. M. de Jussieu profita de cette circonstance pour les disposer suivant une méthode nouvelle qui conserve mieux que toutes les autres les rapports naturels, et dont son oncle Bernard avait eu la première idée, lorsque quinze 
ans auparavantil avaitarrangéle jardin de Trianon.

On substitua à la nomenclature de Tournefort celle de Linné, plus commode et déjà généralement reçue dans toute l'Europe, et l'enseignement de la botanique put dès lors être fait d'une manière régulière. Buffon fit entourer d'une grille de fer l'école et l'orangerie ; il fit démolir un bâtiment qui avait été commencé pour faire une serre au coin de l'orangerie, mais dont la construction ne répondait point au but qu'on s'était proposé, et il en conserva seulement la portion qui soutenait la terrasse de la serre supérieure, sous laquelle on pratiqua des abris pour quelques plantes délicates. Avec les débris de ce bâtiment et les mauvaises terres retirées de l'école, on forma la pente douce qui conduit des allées inférieures au terrain des buttes, et cette montée fut garnie des deux côtés d'un talus planté en ormille, et d'une rampe en fer.

Quelques années après, Buffon entreprit de prolonger le Jardin et de doubler son étendue en y réunissant tous les terrains qui le séparaient de la Seine, et en faisant disparaître les maisons trop rapprochées de l'intendance et de la grande allée méridionale. Cet espace occupé par des plantes potagères appartenait presque en totalité aux religieux de l'abbaye de Saint-Victor. Quelques chan- 
tiers voisins du quai étaient une propriété municipale, que la ville louait à des marchands de bois de construction. Les administrateurs municipaux consentirent à céder ce terrain à Buffon; mais les religieux ne pouvaient vendre les propriétés de l'abbaye. Buffon voulut surmonter cet obstacle, et voici comment il s'y prit.

Il y avait entre le jardin, le boulevard et la rue Poliveau, un vaste enclos dont les maisons et les jardins traversés par la rivière de Bièvre, formaient un domaine appartenant à un seul propriétaire. Buffon l'acheta au mois d'octobre 1779 , pour la somme de I 42,00o livres. Ensuite il proposa aux chanoines de Saint-Victor d'échanger leur terrain contre d'autres de valeur égale, pris dans l'enclos qu'il venait d'acquérir; et, après s'être assuré de leur consentement, il leur fit accorder l'autorisation de cet échange.

Lorsqu'il fut ainsi propriétaire de l'espace qu'il désirait réunir au Jardin, il obtint du roi qu'il voulût bien en faire l'acquisition, et après avoir indemnisé les locataires, il commença les travaux en 1782 . Il fit d'abord démolir les maisons qui gênaient la régularité de son plan, et il employa les matériauxà construire une rue parallèle à la grande allée qui termine le jardin du côté du midi.

Les habitans du quartier donnèrent à cette rue le nom de rue de Buffon, qui lui fut confirmé par 
le corps municipal. On éleva un mur en pierre de taille pour soutenir les terres dans la partie du jardin qui est au-dessus du niveau de la rue; le reste en fut séparé par une grille de fer qui permet à la vue de s'étendre sur les propriétés voisines.

Les limites du Jardin étant ainsi déterminées, on prolongea jusqu'à la rivière les deux allées principales, et on en forma deux autres parallèles le long des clôtures. Celle qui borde la rue de Buffon resta sans plantations du côté du midi pour que les cultures ne fussent pas trop ombragées : l'allée correspondante, dirigée du bas de la petite butte au quai, fut plantée en marroniers d'Inde; le mur de terrasse qui bornait le Jardin au levant fut abattu, et l'on agrandit ainsi l'école et la pépinière.

Entre les deux premières allées, on construisit un vaste bassin carré, creusé jusqu'au niveau de la Seine qui devait lui fournir des eaux par infiltration, et dont les côtés furent plantés de divers arbrisseaux. Un parterre destiné à la conservation et à la propagation des plantes de pleine terre les plus intéressantes, occupa l'espace qui s'étendait entre ce bassin et le quai.

A chacun des deux côtés du jardin et le long des mêmes allées, on pratiqua quatre grands carrés fermés par des treillages. Ceux qui avoisinent la 
rue de Buffon furent plantés en quinconce, des arbres des quatre saisons; ceux qui font suite à l'Ecrle de botanique furent destinés, le premier à une école d'arbres fruitiers, le second au semis des plantes économiques; les deux derniers, qui sont aujourd'hui une école de culture, furent pour le moment disposés en sủpplément de pépinière. Des allées transversales, formées chacune d'une espèce particulière d'arbres, séparèrent ces différens carrés.

Une entreprise aussi vaste dans son ensemble, aussi compliquée dans ses détails, avait paru d'abord exiger beaucoup de temps; mais que ne peut l'esprit d'ordre réuni à l'activité! Quand Buffon eut arrêté son plan, M. Thouin voulut bien se charger seul de surveiller et de conduire des travaux qui s'exécutaient à la fois dans toutes les parties; il dirigea l'emploi des terres et les plantations, il fit niveler le local, creuser les bassins, bâtir les murs d'enceinte, construire la terrasse élevée le long du quai, et tout fut terminé en 1784 .

Cependant le Jardin ne s'étendait point du côté du nord au delà de l'école de botanique et de la petite butte plantée d'arbres verts. Le terrain situé vis-à-vis de l'entrée de l'école à l'extrémité de l'allée des marroniers, appartenant à une compagnie qui voulait y élever des bâtimens, Buffon 
détermina le gouvernement à l'acheter. Comme ce local, plus bas que le jardin et bordé de terrasses, était abrité du nord et de l'ouest, on y transporta les couches destinées aux semis, et à la culture des plantes qui exigent beaucoup de soins, et l'on pratiqua sous l'allée des marroniers qui le sépare de l'école, un passage voûté pour faciliter la communication de l'un à l'autre.

Cette construction ayant été terminée en I 786 , la terrasse sur laquelle on avait établi les couches en 177.4 se trouva libre, et elle fut destinée à l'emplacement d'une serre faite deux ans après, et qui porte le nom de Serre Buffon.

Le Jardin avait été doublé par les nouvelles acquisitions, et l'ordonnance en était magnifique ; on avait tout ce qu'on pouvait désirer pour la culture et l'étude des végétaux, mais on n'en sentait que mieux ce qui manquait pour les autres branches de l'histoire naturelle. Le cabinet ne se trouvait plus assez grand pour les richesses qu'on avait reçues, et l'amphithéâtre situé au fond de la cour, sur une rue très-passagère, était incommode et beaucoup trop étroit pour contenir les élèves qui se rendaient aux divers cours. Toutes les parties du Jardin ayant une destination spéciale, il était indispensable de se procurer un nouveau local. C'est ce qui eut lieu en I 787 par l'acquisition de l'hôtel de Magny, anciennement hôtel de Vau- 
vray, que le roi réunit à l'établissement, d'après la demande de Buffon.

Cet hôtel, accompagné de cours et de jardins , était placé dans l'alignement du mur extérieur des couches, entre la petite butte et la rue de Seine. Sitôt que Buffon l'eut à sa disposition, il fit construire au fond du jardin le vaste amphithéâtre qui sert aujourd'hui pour les leçons, et pour les travaux de la chimie; il transporta dans les appartemens de l'hôtel le logement de MM. Daubenton et de Lacépède, et le second étage du cabinet étant ainsi devenu libre, il le fit arranger de manière qu'on pût y placer des collections. Enfin il obtint du ministre l'autorisation d'ajouter, à lá suite des salles d'histoire naturelle, un bâtiment neuf, de niveau avec l'ancien, et dont chacun des deux étages aurait cinq croisées de face. Ces constructions furent commencées sur-le-champ et continuées avec activité ; mais elles n'ont été terminées qu'après la mort de Buffon.

A mesure que le local devint plus étendu, et que les objets qu'on avait déjà réunis s'y trouvèrent disposés de manière à frapper les regards, on attacha plus de prix aux collections, et celle du Jardin du Roi acquit de la célébrité. Alors plusieurs. particuliers offrirent au cabinet des objets qu'ils aimèrent mieux y voir avec le nom du donateur que de les posséder chez eux; des sociétés savantes 
crurent travailler pour le progrès des connaissances en enrichissant un dépôt public, et des souverains voulant faire au Roi un présent qui lui fût agréable, envoyèrent à son cabinet les doubles de ce qui se trouvait dans le leur.

Ainsi l'académie des sciences ayant acquis la collection d'anatomie de Hunaud, la remit au Jardin pour être jointe à celle de Duverney: le comte d'Angiviller offrit à Buffon son cabinet particulier: les missionnaires établis à la Chine firent parvenir à Buffon ce qu'ils purent se procurer de plus curieux dans un pays où ils avaient seuls le droit de pénétrer : le roi de Pologne envoya une collection de minéralogie très-considérable : enfin l'impératrice de Russie ne pouvant déterminer Buffon à faire le voyage de Saint-Pétersbourg, elle lui demanda son fils; et au retour de ce jeune homme, elle lui fit présent de quelques animaux du nord qui manquaient au cabinet, et de divers objets d'histoire naturelle recueillis dans ses vastes états.

Le gouvernement ne négligea rien non plus pour accroître les trésors d'un monument qui contribuait à la gloire nationale; on ajouta des fonds extraordinaires à ceux qui étaient destinés à l'entretien, et ces fonds furent mis à la disposition de M. Daubenton, afin qu'il pût se procurer et les objets les plus intéressans par leur rareté, el 
ceux qu'il jugerait utiles pour l'étude : on fit venir des arbres étrangers. Le cabinet s'enrichit encore de la collection de zoologie que Sonnerat avait faite dans l'Inde; de celle que Commerson avait faite dans son voyage autour du monde avec Bougainville ; enfin, de celle que Dombey avait rapportée du Pérou et du Chili.

Buffon fit donner des brevets de correspondant du Jardin, avec une pension, à des voyageurs instruits qui s'engageaient à faire des envois pour le jardin et pour le cabinet.

Cependant nous devons à la vérité de l'histoire, de dire que ces collections ne furent point dans le moment fort utiles pour l'étude, et que c'est seulement plus tard et depuis la nouvelle organisation qu'on en a reconnu l'importance, et qu'elles ont été appropriées à leur véritable destination. Buffon n'était point partisan des méthodes; il peignait les animaux de manière à faire connaître leur forme extéricure, leur physionomie, leurs habitudes; il s'élevait ensuite à des considérations générales, mais ill n'aimait point à s'occuper de la subordination des caractères distinctifs et des principes de classification. Il voulait que le cabinet eût de l'éclat, que la curiosité y fût excitée par les contrastes, qu'il présentât comme ses écrits un tableau de ce que la nature offre de plus remarquable, indépendamment des méthodes, qui 
sont l'ouvrage de l'homme. Cette manière de voir avait quelque chose de séduisant pour un génie qui se plaisait à envisager l'ensemble des choses. En effet, dans la nature, où tout paraît en harmonie, les êtres les plus divers sont płacés les uns à côté des autres, et l'esprit saisit à la fois et les rapports qui doivent les unir et les caractères qui les distinguent.

Selon Buffon, le but d'une collection générale était atteint lorsqu'elle captivaitl'attention, lorsqu'elle déterminait à chercher dans la nature ce dont on n'avait vu que des images : on s'exerçait même en démêlant au milieu d'une foule d'objets ceux qui étaient relatifs à l'étude à laquelle on se livrait. Nous ne prétendons point défendre ce système : il ne serait plus admissible aujourd'hui que nous avons assez d'objets pour former des séries, assez de place pour les développer, et que chacune des parties de l'histoire naturelle est étudiée spécialement; il conduisait à négliger ce qui n'offrait pas d'intérêt aux yeux du public. Lorsqu'on recevait un envoi, on choisissait les objets les plus remarquables pour remplir les places vides, le reste était conservé dans des caisses. Daubenton avait fait le squelette de tous les quadrupèdes qu'il avait pu se procurer, et ces squelettes étaient entassés dans une salle où on ne les voyait point. Pour remédier à cet inconvénient, 
on aurait eu besoin d'un local plus vaste, et puisque le cabinet réunissait les productions des trois règnes, il aurait fallu que chacune des collections eût été arrangée par un professeur qui l'eût mise en accord avec ses leçons, et il n'y avait ni professeur de zoologie, ni professeur de minêralogie. Aussi l'école de botanique était-elle la seule partie de l'établissement, ò̀ tout fût disposé d'après une méthode scientifique. Loin de reprocher à Buffon de n’avoir pas exécuté ce qui était alors très-difficile, il faut lui savoir gré d'avoir rassemblé non-seulement la nombreuse collection d'oiseaux qu'il a décrits dans son ouvrage, et celle des poissons dont M. de Lacépède a publié l'histoire, mais une multitude d'objets en tout genre qui, mis en ordre depuis, ont été si utiles au progrès de l'histoire naturelle.

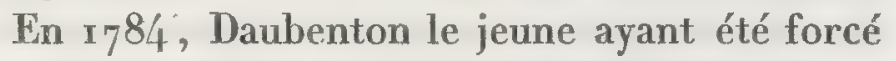
par le mauvais état de sa santé de demander sa démission. de la place de garde et sous-démons trateur du cabinet, Buffon y fit nommer M. de Lacépède que cette nouvelle fonction détermina à s'attacher uniquement à l'histoire naturelle, dans laquelle il s'est depuis illustré comme professeur et comme continuateur de Buffon.

Les relations qu'on avait avec les sociétés savantes, avec les voyageurs, avec les naturalistes de tous les pays, exigeant une correspondance 
suivie à laquelle l'intendant et le garde du cabinet n'auraient pu suffure, et qu'ils ne pouvaient confier à des secrétaires, Buffon obtint en 1787 la création d'une nouvelle place d'adjoint à la garde du cabinet, dont le titulaire serait spécialement chargé de la correspondance. Il fit nommer à cette place M. Faujas de Saint-Fond qui, par sa prodigieuse activité et par la variété de ses connaissances, était très-propre à la bien remplir. Ce savant ne se borna point à entretenir un commerce épistolaire, il fit plusieurs royages, et rapporla au cabinet beaucoup d'objets précieux qu'il avait recueillis ou qu'il avait obtenus en les demandant au nom de Buffon.

Mademoiselle Basseporte avait, pendant trente ans, continué à dessiner pour la collection des vélins tous les objets qui lui étaient indiqués par Bernard de Jussieu. Son zèle ne s'était point ralenti; mais son talent inférieur à celui d'Aubriet, auquel elle avait succédé, était encore affaibli par l'âge. M. de Buffon désirant que la place de peintre du Jardin du Roi fút remplie par l'artiste le plus distingué dans le genre qui convient à l'histoire naturelle, fit donner en I77f la survivance de mademoiselle Basseporte à M. Vanspaendonck, qui se chargea de tout le travail, et devint titulaire en 1780 . Ce choix eut dans la suite des conséquences plus heureuses qu'on ne pouvait le pré- 
JEUXIE M E EOQUL.

voir, puisqu'il a déterminé la création d'une chaire d’iconographie au Muséum.

Le jardin et le cabinet étant ouverts au public, il était essenticl que la police y fût faite avec exactitude. L'autorité nécessaire pour cet objet était donnée à un inspecteur qui avait 4,00o livres d'appointemens et des gardes sous ses ordres. Cette place a été supprimée depuis, lorsque la garde du Muséum a été confiée à une compagnie de militaires vétérans.

Nous venons de voir comment dans l'espace de scize années, Buffon parvint à exécuter les vastes projets qu'il avait conçus en fixant sa résidence au Jardin. Il nous reste à dire un mot du progrès qui eut lieu dans l'enseignement. Ce progrès ne dépendait pas directement de lui, mais le choix qu'il fit des professeurs en est la principale cause, et c'est encore un titre à notre reconnaissance.

Il n'y avait de chaires au Jardin du Roi que pour la botanique, la chimie et l'anatomie; mais comme M. Daubenton, ou le sous-démonstrateur qui lui était adjoint se rendait tous les jours au cabinet, les naturalistes pouvaient demander l'explication de ce qui était sous leurs yeux, et ces leçons particulières étaient d'autant plus utiles qu'elles étaient appropriées au degré d'instruction de ceux à qui elles s'adressaient.

Lemonnier avait la place de professeur de bo- 
tanique depuis 1758 , et Bernard de Jussieu celle de démonstrateur depuis I722. Mais le premier étant obligé de se rendre à Versailles; et le sécond se trouvant affaibli par l'âge, ils avaient choisi pour leur suppléant M. de Jussieu le neveu, qui était ainsi chargé de faire les leçons dans le jardin et les herborisations à la campagne. Pendant les trois dernières années de sa vie, Bernard de Jussicu se reposa entièrement des détails de la culture sur M. Thouin; ce fut pour lui une grande jouissance de voir la replantation de l'école. Lorsqu'il venait se promener au Jardin, ses anciens élèves accouraient auprès de lui; ils l'entouraient avec respect et recueillaient ses moindres paroles. Parmi les nombreux services qu'il a rendus, il faut surtout compter celui d'avoir formé M. de Jussieu, qui a fait de la botanique un corps de science régulier, en développant et perfectionnant la méthode naturelle dont son oncle avait eu la première idée. Lorsqu'il termina son honorable carrière en I 777, à l'âge de soixante-dix-huit ans, ce même neveu fut nommé à sa place (I).

(1) Bernard de Jussieu n'a inséré qu'un petit nombre de Mémoires dans le récuél de l'Académie des Sciences, dont il avait été nommé membre à l’age de 26 ans. Plus óccupé des progrès de la science que de sa propre réputation, il répandait ses découvertes en les communiquant sans réserve à ses élèves; et c'est ainsi qu'il s'est acquis des droits a la reconnaiousance des naturalistes. 
Lemonnier était extrêmement satisfait de la manière dont M. de Jussieu faisait son cours; mais il prenait trop d'intérêt à l'établissement pour ne pas désirer que les deux places de professeur et le démonstrateur attachassent au Jardin deux savans qui travailleraient de concert au progrès de la botanique. Il forma donc le projet de se retirer avec le titre de professeur honoraire aussitôt qu'il serait sûr de faire entrer au Jardin un homme capable de remplir ses vues. Il avait jeté les yeux sur M. Desfontaines, dont il appréciait le mérite, et dont il prévoyait la réputation; mais ce jeune savant n'étant point encore assez connu, Lemonnier jugea convenable d'attendre, et ce ne fut qu'en 1786 qu'il fit les démarches nécessaires pour réaliser son projet. A cette époque, M. Desfontaines était de retour de son voyage en Barbarie, d'où il avait rapporté les plantes dont il a publié l'histoire; il avait fixé l'attention des botanistes par d'excellens mémoires, et, depuis trois ans, il avait été reçu membre de l'académie des sciences. M. Lemonnier le proposa à Buffon, quiapplaudit à ce choix. M. Desfontaines voulait occuper seulement la place de démonstrateur, et laisser celle de professeur à M. de Jussieu; mais ce dernier préféra conserver des forictions que son oncle avait exercées pendant cinquante-cinq ans. 
HISTOIRE DU MUSÉUM.

Lorsque M. Desfontaines fut nommé, l'école était déjà fort riche, et les plantes y étaient disposées dans l'ordre des affinités. L'enseignement n'était plus borné à la démonstration des plantes médicinales, et les progrès que la science avait faits depuis Tournefort par les travaux de Linné, d'Adanson et de Jussieu, autorişaient à lui donner une паache plus philosophique.

M. Desfontaines fut le premier à juger que pour bien apprécier les caractères qui distinguent les genres et les espèces, il faut avoir une connaissance générale de la nature des végétaux, des fonctions propres à chacun des organes qui les composent et des phénomènes qu'ils présentent aux différentes périodes de leur développement. Il résolut en conséquence de diviser son cours en deux parties: la première consacrée à l'anatomie et à la physiologie végétale, la seconde à la classification et à la description des familles, des genres et des espèces. Cette innovation fit époque dans l'enseignement de la botanique, qui dès lors ne fut plus seulement la connaissance des formes extérieures des plantes, mais encore celle de leurs rapports, de leurs usages et des diverses modifications dont elles sont susceptibles. C'est à la direction donnée à l'étude dans les cours du Jardin du Roi depuis I 788 que sont dus les travaux qui ont fait de la physiologie végé- 
tale non plus une partie accessoire, mais la base fondamentale de la botanique, et qui nous ont conduits à faire l'application des principes de cette science à. l'agriculture et aux arts.

M. Desfontaines continua pendant quelques années à démontrer les plantes dans l'école; mais. sa manière d'enseigner attira un si grand nombre d'élèves, qu'il fut désormais impossible que tous pussent entendre le professeur en se trouvant. placés sur une ligne droite le long des platesbandes. Le professeur prit alors le parti de faire ses leçons dans l'amphithéâtre, où l'on porte des échantillons que tout le monde peut voir, en ayant soin de placer à l'école et à leur étiquette toutes les plantes qui appartiennent à la famille dont il a été question dans la leçon. Ainsi chacun peut aller les examiner à loisir, vérifier les caractères que le professeur a fait remarquer et comparer même chaque plante avec la description qu'on en trouve dans les livres.

Tandis que M. Desfontaines enseignait ainsi l'ensemble de la science, en parcourant successivement toutes les familles du règne végétal, M. de Jussieu faisait chaque semaine une herborisation à la campagne. Dans ces promenades, il ne se bornait point à nommer en passant les espèces qu'on lui présentait; il profitait de toutes les occasions pour faire remarquer les caractères 
qui réunissent et ceux qui distinguent les différens groupes de végétaux, et il exerçait ainsi les élèves à faire sur les plantes indigènes qui s'offraient sans ordre à leurs yeux, l'application des principes qu'il a développés depuis dans un ouvrage devenu classique aussitôt après sa publication.

La méthode introduite au Jardin du Roi ayant été suivie dans les diverses écoles du royaume, et ensuite adoptée dans les pays étrangers, et l'exposition des familles naturelles ayant coordonné les faits, le nombre prodigieux de plantes qu'on décourre tous les jours ne se présentent plus comme isolées, elles viennent remplir les lacunes qui se trouvent encore dans la série générale.

L'enseignement de la chimie continua d'être confié à deux professeurs, dont le premier exposait la théorie, et le second faisait les diverses opérations. Nous verrons bientôt que ces deux parties de la science n'auraient dù ètre séparées qu'autant qu'on aurait considéré la première comme destinée à exposer les principes et à faire les expériences qui en prouvent la vérité, et que l'autre aurait enseigné l'application de la chimie aux arts, comme cela se fait aujourd'hui.

Macquer, suppléant de Bourdelin slepuis I 77 ot professeur titulaire en $\mathbf{1 7 7 7}$, s'écarta entièrement des idées de ses prédécesseurs. Il développa la 
théorie de Becker et de Stahl, que Rouelle avait fait connaître, et à laquelle il avait déjà fait un si grand nombre de partisans. Il suffit de lire le Dictionnaire de chimie de Macquer pour se convaincre qu'un homme d'un esprit aussi juste, et qui avait autant de netteté dans les idées, dut traiter son sujet avec plus de méthode et d'exactitude qu'on ne l'avait fait jusqu'alors. Il ne cherchait point à frapper l'imagination de ses auditeurs, mais à fixer leur attention sur les grands phénomènes pour les conduire à en découvrir d'euxmêmes l'explication. Quoiqu'il professât la doctrine de Stahl conjointement avec les démonstrateurs, il fut frappé des découvertes de Lavoisier, il pressentit même les changemens qu'elles devaient produire, et loin d'en repousser les conséquences, il prépara ses élèves à les adopter. A sa mort, arrivée en 1784 , Buffon choisit pour le remplacer MI. de Fourcroy, qui n'avait pas atteint sa trentième année, et qui n'était pas encore membre de l'académie, mais qui s'était déjà fait une grande réputation par les talens qu'il avait montrés en suppléant dans ses cours Bucquet son mâ̂tre, professeur à l'École de médecine.

C'est de l'époque où M. Fourcroy entra au Jardin qu'il faut dater la propagation de la nouvelle théorie chimique, et l'on peut assurer que cette théorie, qui a changé la face de la science, ne se 
serait répandue que lentement s'iln’eût été chargé de la développer.

Les découvertes de Cavendish sur la décomposition de l'eau, de Lavoisier sur la combustion, avaient fait sentir la nécessité d'une nomenclature qui, distinguant les corps simples des corps composés, indiquât en même temps la nature des élémens de ces derniers. Ce projet, conçu par Guyton de Morveau, fut adopté par Lavoisier et par quelques autres chimistes, du nombre desquels était Fourcroy, 'et il fut exécuté avec un esprit de méthode dont les sciences n'avaient point encore offert une semblable application : mais quoique cette nomenclature, dans laquelle chaque mot offrait l'explication d'un fait, dût épargner un long travail à ceux qui voulaient connaitre non-seulement tous les composés existans, mais encore tous ceux qui sont possibles; c'était toujours une langue nouvelle, et rien n'est aussi difficile que de faire adopter un langage différent de celui auquel on s'est accoutumé. La chose était si utile, que leshommes studieuxn'auraient pu s'y refuser ; mais s'ils n'eussent appris cette langue que dans les livres, elle ne serait jamais devenue d'un usage universel. Fourcroy la parla dans ses cours, et comme son éloquence captivait ses auditeurs, comme la clarté de ses idées se réunissait à une diction également précise et harmonieuse, 
comme tout ce qu'il disait restait gravé dans la mémoire, on ne s'entretint plus de chimie sans employer les termes dont il se servait. Le même langage étant adopté par le démonstrateur devint bientôt vulgaire, et c'est vraiment aux cours fails au Jardin du Roi, que la chimie doit ses rapides progrès. Rouelle avait enflammé l'imagination de ses élèves, en présentant des merveilles, mais il n'avait point une méthode qui pût d'elle-même classer, distinguer et coordonner les faits. Fourcroy excita le même enthousiasme; il dirigea vers les études chimiques une multitude de bons esprits. En exposantles découvertes de ses contemporains, en proclamantle nom de ceux quiles avaient faites, en annonçant toutes les conséquences qui devaient en résulter, il se fit bientôt une réputation qui s'étendit hors de sa patrie. Des princes étrangers entretenaient à Paris des jeunes gens pour suivre ses cours, et rapporter dans leur pays la doctrine qu'ils y auraient apprise; les gens du monde venaient l'écouter pour jouir de son éloquence, et se passionnaient pour une science qui leur était présentée d'une manière si agréable. L'amphithéâtre où il faisait ses leçons ne pouvait contenir tous ccux qui venaient pour l'entendre, et l'on fut deux fois obligé de l'agrandir.La langue qu'il avait enseignée s'est enrichie et perfectionnée, parce que la découverte d'un grand nombre de substances 
HISTOIRE DU MUSÉUM.

simples a fait módifier les noms des différens composés; mais les principes en sont toujours les mêmes. Le goût qu'il avait inspiré pour la chimie s'est successivement accru; plusieurs chimistes distingués se sont formés à son école, et les professeurs qui soutiennent aujourd'hui la réputation des deux chaires du Jardin sont ses élèves.

Rouelle le jeune, qui était démonstrateur titulaire depuis I $77^{\circ}$, étant mort en 1779 , sa place fut donnée à M. Brongniart qui, déjà professeur à l'Ecole de pharmacie, avait publié un ouvrage sur les procédés de la chimie, et qui travaillait au Journal des sciences, arts et métiers. M. Brongniart, qui s'était d'abord conformé aux vues de Macquer, adopta bientôt les idées nouvelles. Il répéta dans l'amphithéâtre toutes les expériences qui prouvaientla théorie du professeur, en employant la nouvelle nomenclature.

Antoine Petit avait, par son éloquence, conservé à la chaire d'anatomie du Jardin l'éclat qu'elle avait reçu de Duverney et de Winslow. Il royait à chacun de ses cours s'accroître le nombre de ses élèves : cependant, après quelques années, il mit moins d'exactitude à faire ses lecons, et l'on en murmura d'autant plus qu'on était plus empressé de l'entendre. Il se fit alors suppléer par Vicq-d'Azir, qui était à tous égards digne de lui succéder, et voulut lui faire donner sa sur- 
vivance. Mais M. de Buffon pensa que cette place était due à M. Portal, qui, dix ans auparavant, avait fait des leçons pour Ferrein, et s'était depuis acquis une grande réputation : M. Portal fut donc nommé, en $177^{8}$, à la chaire qu'il remplit encore aujourd'hui.

Quoique Vicq-d'Azir n'ait suppléé Ferrein que pendant deux ans, il a des droits à notre reconnaissance, non-seulement pour le talent qu'il déploya dans ses leçons, mais parce qu'il répandit des idées très-justes sur l'anatomie comparée, qui depuis; professée spécialement au Muséum, est devenue la base de la zoologie.

Le tableau que nous avons tracé de ce qui fut fait pour l'établissement depuis $\mathbf{I} 77^{2}$, jusqu'à 1788 , est aussi celui des obligations que nous avons à Buffon. Ce grand homme, en répandant universellement le goût de l'histoire naturelle par ses immortels écrits, voulut donner à ceux dont il avait enflammé le zèle, la facilité d'étudier les objets qu'il avait si bien peints. Il conçut en même temps et le plan de son Histoire naturelle et celui de l'agrandissement ou plutôt de la création du Muséum. Il médita ce plan pendant plusieurs années, parce qu'il voulait que les diverses parties formassent un ensemble, et que toutes fussent susceptibles de perfectionnemens successifs. Ce ne fut qu'après s'être assuré les moyens de réa- 
liser ses projets, qu'il en commença l'exécution. Dès lors aucun obstacle ne fut capable de l'arrêter, aucun travail ne put lasser sa patience: son entreprise fut couronnée par le succès le plus éclatant, et sur la fin de sa vie il eut lieu de s'applaudir des sacrifices qu'il avait faits. Le Jardin et le Cabinet du Roi, furent avec raison cités comme son ourrage, comme la plus belle institution qu'on eût jamais formée pour le progrès des sciences, comme un point central où devaient se rendre de diverses contrées tous ceux qui se livrent à l'étude de la nature. Si les Français se glorifiaient d'un tel monument; les étrangers l'admiraient sans jalousie, parce qu'il était également utile aux hommes de toutes les nations.

Cependant l'établissement était loin encore de ce qu'il est devenu quelques années plus tard, et les collections paraîtraient peu de chose si on les comparait à ce qu'elles sont aujourd'hui; mais les cadres étaient formés, l'impulsion était donnée, et tout était disposé de manière à préparer de nouveaux accroissemens. Si ces accroissemens n'ont eu lieu qu'après Buffon, et lorsque plusieurs savans, tous animés du même esprit, ont été chargés d'administrer chacun une partie, cela ne diminue rien de sa gloire; car sans lui la nouvelle organisation ne serait point devenue nécessaire, et le Muséum d'Histoire naturelle n'existerait pas. 



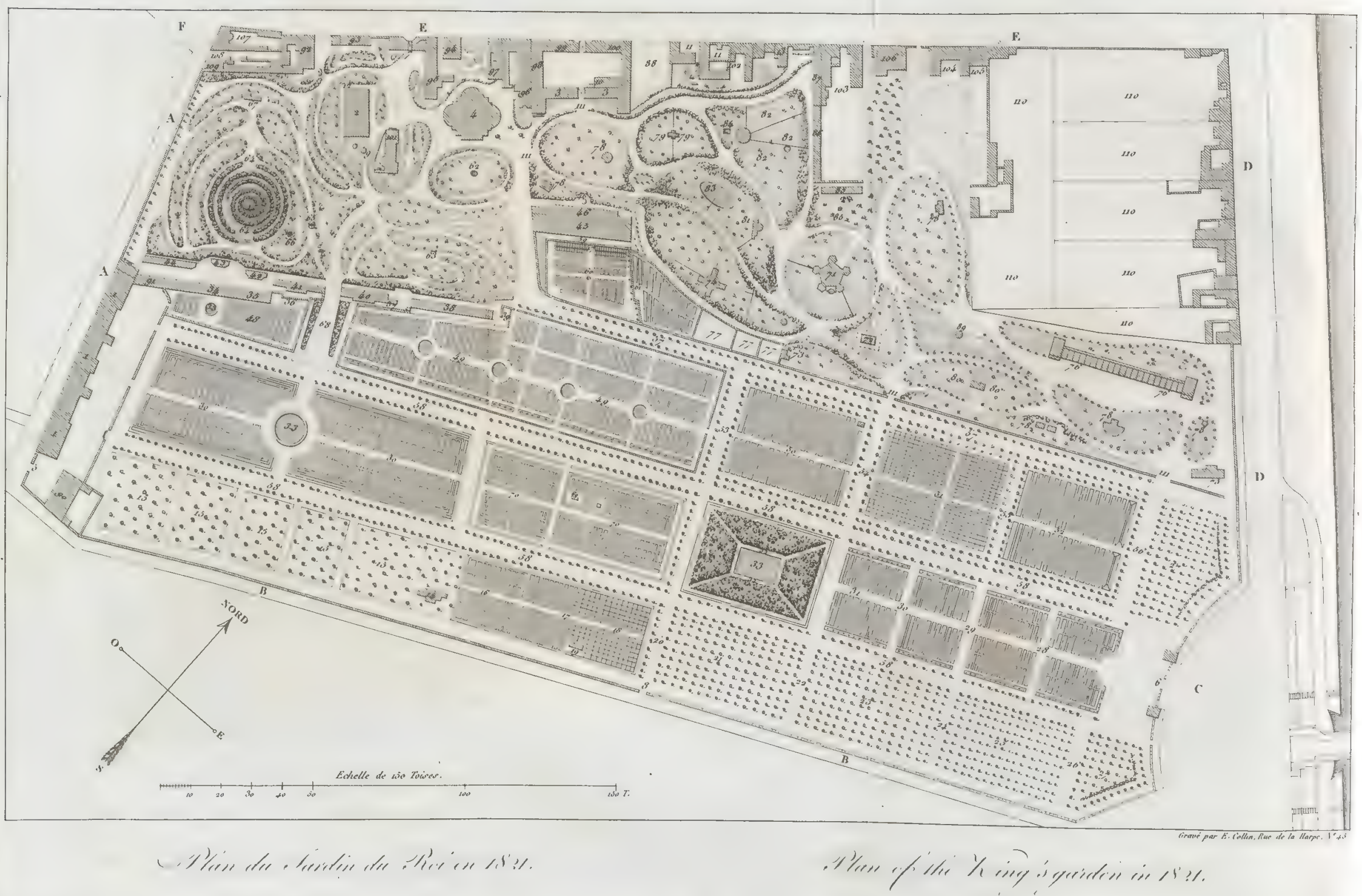




\section{TROISIÈME ET DERNIÈRE ÉPOQUE,}

FEPUIS LA MORT DE BUFFON JUSQU'AU TEMPS ACTUEL.

NOUVELLE ORGANISATION.

A LA mort de Buffon, le $x 6$ avril I $_{7} 88$, la place d'intendant du Jardin passa, non point à M. lc comte d'Angiviller qui en avait la survivance, mais à son frère M. le marquis de la Billarderie. Celui-ci fit continuer les travaux commencés par Buffon. On lui doit de plus la construction de la petite serre des ficoïdes. Ce fut lui qui voulant attacher à l'établissement M. 'le chevalier de la Marck, déjà si célèbre par sa Flore française, fit créer pour lui la place de botaniste du cabinet, en le chargeant de conserver et d'arranger les herbiers. Son caractère conciliant le fit aimer de tous les professeurs; il ne négligea rien pour favoriser l'enseignement et pour entretenir l'ordre qui existait; mais son crédit était peu de chose en comparaison de celui dont Buffon avait joui. La cour étant occupée de réduire toutes les dé- 
penses, l'intendant du Jardin ne pouvait guère demander au ministre des fonds extraordinaires. L'établissement était d'ailleurs devenu trop considérable pour que le régime auquel il était soumis du temps de Buffon pût lui convenir.

Le 20 août 1790 ; M. Lebrun fit au nom du comité des finances de l'assemblée constituante un rapport sur le Jardin du Roi, dans lequel il évaluait la dépense de cetétablissementà 92,222 liv. dont 12,777 pour l'entretien. Ce rapport, qui fut le premier signal d'une nouvelle organisation, commence par ces mots :

"Le Jardin du Roi doit être sous l'administra" tion immédiate du roi, mais la nation ne peut "le voir sans intérêt, et c'est sur le trésor public "que la dépense doit être affectée. "

Le rapporteur lut ensuite un projet de décret en sept articles, dans lequel il proposait la réduction desappointemens de l'intendant de I2,000 à 800 o livres; la suppression de plusieurs places, et particulièrement de celle de commandant de la police du Jardin, ce service devant être fait par des invalides; l'augmentation du traitement de quelques professeurs; l'établissement d'une chaire d'histoire naturelle aux appointemens de rooo livres, etc.

Pendant la discussion sur les divers articles de ce projet, le président de l'assemblée reçut une 
adresse de MM. les officiers du Jardin du Roi. C'était ainsi qu'on nommait les gardes et les démonstrateurs, du cabinet, les professeurs et le peintre attachés à l'établissement. L'assemblée ayant approuvé les vues sages de cette adresse, en ordonna le renvoi au comité des finances, en ajournant le rapport définitif, et en invitant les officiers du Jardin à présenter un projet de règlement pour fixer l'organisation d'un établissement si utile.

En conséquence de cette décision, M. de la Billarderie ayant convoqué les officiers du Jardin du Roi, ils se formèrent en assemblée, et après avoir nommé M. Daubenton président et M. de Lacépède secrétaire, ils rédigèrent le projet qui leur était demandé. Ce projet fut signé non-seulement par les membres qui étaient en fonction, mais encore par MM. Petit et Lemonnier, qui avaient professé précédemment et qui s'étaient retirés avec le titre de professeurs honoraires.

Les articles du règlement sont à peu près les mêmes qui furent admis trois ans plus tard. Pendant cet intervalle, les officiers du Jardin cessèrent de se réunir et de s'occuper d'affaires administratives. Chacun d'eux se borna à remplir ses fonctions, et avec tant de zèle que plusieurs faisaient un nombre de leçons double de celui qui 
leur était prescrit, et donnaient encore après les cours des explications aux élèves qui venaien $t$ les consulter. C'était tout ce qu'ils pouvaient faire pour dirigerles jeunes gens vers l'étude des sciences naturelles, dont les résultats sont d'une utilité générale, et pour les détourner des théories politiques qui, lorsqu'elles ne sont pas fondées sur l'expérience, deviennent subversives de l'ordre social.

Les troubles ayant commencé, M. de la Billarderie ne crut pas prudent de rester en France, et la place d'intendant se trouvant vacante; le Roi y nomma M. Bernardin de Saint-Pierre, au mois de juillet 1792 .

M. de Saint-Pierre eut la direction du Jardin du Roi dans des conjonctures bien difficiles. Écrivain distingué, doué du talent de peindre la nature, d'émouvoir la sensibilité, d'inspirer des affections douces, d'agir à la fois sur le cour et sur l'imagination, il manquait de notions exactes dans les sciences, et son caractère timide et mélancolique le rendait étranger à cette connaissance des hommes et des affaires, à cette énergie qui mettent à même d'exercer l'autorité. C'était précisément l'homme qui convenait à l'établissement dans cette époque d'un bouleversement général. Sa simplicité, sa vie retirée contribuèrent peut-être à le garantir des persécutions dont 
étaient menacés tous les hommes qui occupaient un poste éminent, et sa sagesse fut très-utile. Il s'occupa des détails du Jardin; il eut soin de ne faire aucune démarche, aucune proposition, sans s'être concerté avec ceux qui l'habitaient, et il envoya au ministre, sur les améliorations qu'on pourrait faire, plusieurs mémoires dans lesquels on trouve des vues très-saines et un esprit d'économie que les circonstances rendaient alors nécessaire. Dans ces mémoires, dont nous avons la copie, on voit toujours ces paroles : après avoir consulté les anciens. C'est ainsi qu'il nommait les personnes qui étaient depuis longtemps attachées à l'établissement, mais qui n'avaient encore aucune part à l'administration. En réduisant les dépenses inutiles, il trouvait le moyen de pourvoir à celles dont il reconnaissait l'importance. Il fit construire une serre, qui faisant suite à celle du Cierge adossée à la butte, aboutit à la terrasse élevée au-dessus de la rue, à l'extrémité du Cabinet. Cette serre porte aujourd'hui. son nom.

La ménagerie de Versailles ayant été abandonnée, on voulut se débarrasser des animaux qui s'y trouvaient, et M. Couturier, régisseur des domaines du roi dans cette ville, écrivit à M. de Saint-Pierre de la part du ministre pour les lui offrir : celui-ci nayant ni un local où il pût les 
placer, ni les fonds nécessaires pour payer leur nourriture, obtint de M. Couturier qu'il consentît à en prendre soin pendant quelqué temps; et il adressa au gouvernement un mémoire sur la nécessité d'établir une ménagerie au Jardin. Ce mémoire fit beaucoup de sensation; il détermina à prendre des mesures pour que les animaux fussent conservés, et quoiqu'ils n'aient été transportés au Muséum que dix-huit mois plus tard et lorsque la place d'intendant avait été supprimée, c'est à M. de Saint-Picrre que nous devons la création de notre ménagerie.

Le I 8 août I 792, un décret de l'assemblée législative ayant supprimé les universités, les facultés de médecine et les corporations savantes, on eut tout lieu de craindre que le Jardin du Roi ne fût enveloppé dans la même proscription. Mais comme il était réputé propriété nationale, et que tous ceux qui venaient le visiter y étaient également accueillis; comme le peuple le croyait principalement destiné à la culture des plantes médicinales, et que le laboratoire de chimie était considéré comme un atelier pour faire du salpêtre, tout y fut respecté.

Cependant une faction ennemie de l'ordre et de tout gouvernement, devenue redoutable par sa victoire du 3 I mai, voulait anéantir tout ce qui rappelait les souvenirs de la monarchie. Un éta- 
TROISTÈ ÉPOQUE.

73

blissement dont les employés avaient été nommés par le roi, devait être l'objet de sa fureur. Le péril était imminent; et il eût été impossible d'y échapper s'il ne se fút pas trouvé dans la Convention quelques hommes de courage, qui, reconnaissant enfin l'abîme dans lequel ils étaient entraînés, désiraient arrêter ce torrent dévastateur, et préparer un retour vers le bien en conservant les institutions utiles aux sciences et aux arts. Parmi eux il faut surtout distinguer M. Lakanal, qui, en sa qualité de président du comité d'instruction publique, exerçait une grande influence. Aussitôt qu'il fut informé du danger qui nous menaçait, il se rendit secrètement au Jardin, et s'entretint avec MM. Daubenton, Thouin et Desfontaines, sur les moyens de le prévenir. Il se fit remettre par eux le projet de règlement qu'ils avaient présenté à l'assemblée constituante, et dès le lendemain il fit rendre un décret qui constituait et 'organisait l'établissement; , en lui donnant le titre de Muséum d'histoire naturelle.

Ce décret rendu le ro juin, et publié le I4, se compose de quatre titres, dont nous allons rapporter les articles essentiels.

TITRE $\mathbf{I}^{\text {er. }}$

"L'établissement sera nommé à l'avenir Muséum d'histoire naturelle. 
"Son but sera l'enseignement de l'histoire naturelle dans toute son étendue.

"Tous les officiers du Muséun porteront le titre de professeurs, et jouiront des mêmes droits.

"La place d'intendant sera supprimée, et le Iraitement attaché à cette place sera également réparti entre les professeurs.

"Les professeurs nommeront chaque année, au scrutin, un directeur et un trésorier, choisis par eux; le directeur ne pourra, après l'expiration de l'année, être continué que pour un an; il présidera l'assemblée, et sera chargé de faire exécuter les délibérations.

“Lorsqu'une place de professeur sera vacante, les autres professeurs y nommeront le savant qu'ils jugeront le plús propre à la remplir (r).

(1) Cet article qui donnait a l'assemblée des professeurs le droit de nommer aux places vacantes, fut abrogé par la loi sur l'instruction

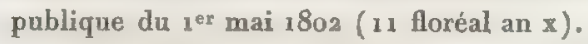

L'article 24 de cette loi est ainsi conçu :

" Quand il vaquera (dans les écoles spéciales) une place de professeur, il y sera nommé par le premier consul, entre trois candidats qui seront présentés, le premier par une des classes de l'institut national, le second par les inspecteurs généraux des études, et le troisième par les professeurs de l'école où la place sera vacante. „

Depuis l'organisation de l'université, an mois de mai 1808 , les ins * pecteurs soumis au grand-maitre nont plus eu le droit de coopérer aux nominations. Aujourd'hui lorsqu'il $\mathrm{y}$ a une chaire vacante au Muséum, le roi y nomme sur la présentation de l'assemblée des professeurs, et sur celle de l'académie des sciences. On juge bien que le choix fait par les professeurs doit presque toujours déterminer celni de l'académie. 
T I T R E II.

"On donnera dans le Muséum douze cours, savoir :

$I^{\circ}$ Un cours de Minéralogie ;

$2^{\circ}$ de Chimie générale:

$3^{\circ}$ des Arts chimiques;

$4^{\circ}$ de Botanique dans le Muséum ;

$5^{\circ}$ de Botanique dans la campagne;

$6^{\circ} \quad$ de Culture ;

7 et $8^{\circ}$ deux cours de Zoologie;

$9^{\circ} \quad d^{\prime}$ Anatomie humaine;

$10^{\circ}$ d'Anatomie des animaux;

I I $^{\circ}$ de Géologie;

$\mathrm{I}^{\circ}$ d'Iconographie naturelle.

"La nature des objets qui doivent être traités dans ces cours, et les détails relatifs à l'organisation particulière du Muséum, seront l'objet d'un règlement que les professeurs sont chargés de rédiger, et qu'ils communiqueront au comité d'instruction publique. »

Le titre III ordonne qu'il y aura au Muséum une bibliothèque où l'on réunira les livres d'histoire naturelle qui se trouvent dans les dépôts appartenans à la nation, les doubles de ceux qui sont à la grande bibliothèque nationale, et la collection des plantes et animaux peints d'après nature, qui est déposée dans la même bibliothèque.

Le titre IV porte que le Muséum sera en corres- 
$7^{6}$ HISTORE DU MUSÉU.

pondance avec tous les établissemens analogues, placés dans les départemens.

Dans le décret dont nous venons d'extraire les principaux articles, douze chaires sont établies, sans qu'on désigne par leur nom ceux qui doivent les remplir. Il est seulement dit qu'elles. le seront par les douze officiers du Jardin: on laisse à ceux-ci le soin de distribuer entre eux les fonctions.

Ces douze officiers étaient:

MM. Daubenton, garde du cabinet et professeur de minéralogie au collége de France.

Fourcroy, professeur de chimie.

Brongniart, démonstrateur de chimie.

Desfontaines, professeur de botanique.

De Jussieu, démonstrateur.

Portal, professeur d'anatomie.

Mertrud, démonstrateur.

La Marck, botaniste du cabinet, chargé desherbiers.

Faujas de Saint-Fond, adjoint à la garde du cabinet, chargé de la correspondance.

Geoffroy, sous-garde et sous-démonstrateur du cabinet, élève de M. Daubenton pour la zoologie.

Vanspaendonck, peintre du cabinet.

A. Thouin, jardinier en chef.

Il $n^{\prime} y$ avait aucune difficulté pour ceux qui étaient déjà professeurs ou démonstrateurs, mais 
TROISIÈ ME ÉPOQUE:

MII. Faujas et de la Marck n'étaient point dans ce cas : la correspondance appartenant désormais à l'assemblée, et les herbiers ayant été mis par le décret dans les attributions du professeur de botanique, l'un et l'autre se trouvaient sans fonctions. Le premier, très-connu par ses voyages et par son bel ouvrage sur les volcans du Vivarais, fut nommé à la chaire de géologie; et M. de la Marck, qui s'était également occupé de zoologie et de botanique, et qui avait la réputation d'être l'homme de France qui connaissait le mieux les coquilles, fut chargé d'enseigner l'histoire des animaux sans vertèbres. Cette nouvelle direction donnée à ses travaux nous a procuré d'excellens ouvrages.

On sentait la nécessité de diviser en trois parties l'enseignement de la zoologie; mais comme IM. de Lacépède avait depuis quelques mois donné sa démission de laplace de garde et sous-démonstrateur du cabinet, pour se retirer à la campagne, on n'osa le proposer, et l'on ne parla point de cette troisième chaire, parce qu'on se flattait que celui qui y avait des droits serait rappelé dans des circonstances plus favorables; ce qui eut lieu en effet. En attendant, M. Geoffroy, qui avait succédé à M. de Lacépède dans la place de sous-démonstrateur du cabinet, se chargea seul d'enseigner l'histoire des quadrupèdes et des oiseaux, et celle des poissons et des reptiles. 
Ce fut le g juillet 1793 , que les professeurs, ayant recu la notification de ce décret, se formèrent en assemblée et nommèrent M. Daubenton président, M. Desfontaines secrétaire, et M. Thouin trésorier. Depuis cette époque, ils se réunirent à des jours fixes, et rédigèrent le règlement supplémentaire qui leur avait été demandé.

La première, chose dont ils s'occupèrent fút d'obtenir la création de quelques emplois que la nouvelle organisation rendait nécessaires.

L'administration générale du cabinet appartenant à l'assemblée, et le soin de former et d'arranger les collections de minéralogie, de botanique, de zoologie, d'anatomie, etc., entrant dans les fonctions du professeur qui devait enseigner la partie de l'histoire naturelle à laquelle ces collections sont relatives; on avait supprimé les places de garde du cabinet et celle d'adjoint à la garde du cabinet, qui étaient remplies par MM. Daubenton, Faujas et Geoffroy. Mais il fallait que quelqu'un fût chargé de garder les clefs des galeries, de veiller à la conservation des objets, et d'introduire les personnes qui venaient visiter le cabinet, soit pour s'instruire, soit pour en admirer les richesses. Cesfonctions furent données à M. Lucas, qui avait passé sa vie dans l'établissement, et en qui M. de Buffon avait beaucoup de confiance. M. André Thouin, déjà membre de l'académie des 
sciences, étant devenu professeur de culture, M. Jean Thouin, son frère, fut nommé jardinier en chef. On créa aussi quatre places d'aides naturalistes (I) pour fáire, sous les professeurs, les travaux nécessaires à la préparation et à l'arrangement des collections, et l'on attacha au Muséum trois peintres d'histoire naturelle : MM. Maréchal, Redouté l'aîné, et Redouté le jeune. Ces nominations furent faites par l'assemblée et les choix approuvés par le gouvernement.

En même temps on disposa le local de la bibliothèque, de manière à ce qu'il pût recevoir des livres choisis dans les dépôts publics, ainsi que les soixante-quatre portefeuilles de vélins représentant des plantes et des animaux dont la continuation fut ordonnée. Le placement de ces vélins au Muséum était non-seulement une chose convenable pour l'étude de l'histoire naturelle, c'était encore un acte de justice ; car tous avaient été exécutés par les peintres du Jardin, et payés sur ses fonds; et on ne les avait déposés à la bibliothèque du roi, que parce qu'il n'y avait point encore de bibliothèque au Jardin.

Au mois de juillet 1794 , le local se trouvant prêt à recevoir les livres, dont le choix avait été fait par

(1) Elles furent remplies par MM. Desmoulins, Dufresne, Valenciennes et Deleuze; les deux premiers poux la zoologie, les deux antres pour la minéralogie et la botanique. 
M. de Jussieu, M. Toscan fut nommé bibliothécaire. On lui adjoignit le mois suivant, M. Mordant de Launay, et la bibliothèque fut ouverte au public le 7 septembre 1794 .

Les animaux de la ménagerie de Versailles, de celle du Rincy, et d'autres appartenant à des particuliers qui faisaient, métier de les montrer au public, ayant été transportés au Muséum dans les premiers mois de 1794 , on pratiqua, sous les galeries du cabinet, des loges pour ceux qui deraient être renfermés : les autres furent placés dans des bosquets le long de la rue de Buffon, ou dans des écuries ; et comme il était essentiel de débarrasser promptement le rez-de-chaussée du cabinet, on fit arranger un petit bâtiment situé à l'extrémité de l'allée des marroniers, et l'on en fit une ménagerie provisoire particulièrement destinée aux animaux féroces.

On disposa la maison dite l'intendance pour y loger des professeurs, on arrangea les salles du cabinet, et l'on décida qu'on construirait de nouvelles galeries au deuxième étage. Enfin, un arrêté du comité d'instruction publique, du mois de septembre 1794, ordonna l'acquisition de la maison et des terrains qui bornaient le Muséum du côté du nord-ouest, acquisition dont la nécessité avait déjà été reconnue par l'assemblée constituante. 
Pendant ce temps, les professeurs rédigèrent lc règlement qui leur avait été demandé, et ce ful. en conséquence de ce règlement, que $\mathbf{M}$. Thibaudeau fit au nom du comité d'instruction publique un rapport dont les conclusions furent adoptées, et qui fixa l'organisation du Muséum telle qu'elle est aujourd'hui; sauf quelques légères modifications que les circonstances ontrendues nécessaires.

La loi du i I décembre I 794, rendue d'après le rapport du comité d'instruction publique, créa une troisième chaire de zoologie, à laquelle M. de Lacépède fut nommé. Cette loi donne aux professeurs toute l'administration de l'établissement: elle porte à 5,ooo francs leurs appointemens qui n'étaient que de 2,800 livres; elle fixe à 194,000 francs la dépense de l'année suivante; elle ordonne que les terrains compris entre la rue Poliveau, la rue de Seine, la rivière, le boulevard de l'Hôpital et la rue Saint-Victor seront réunis au Muséum, et qu'on s'occupera de l'estimation de ces terrains et de l'emploi qu'il convient d'en faire.

La commission d'instruction publique avait formé pour l'agrandissement du Muséum, un plan encore plus vaste, mais qu'il était impossible de réaliser ; et un arrêté du comité de salut public, du mois de mai I 794, l'avait converti en loi. Cette loi fut ensuite rapportée à la sollicitation même des professeurs, et l'on décida que l'étendue du 
Muséum serait bornée par les rues de Buffon ct de Seine.

Kien n'était plus sage que de se renfermer dans ces limites, dont l'aspect des lieux et le but de l'établissement indiquent la convenance; mais comme on avait négligé de profiter d'une circonstancefavorable pour se procurer par voie d'échange tout ce qui était compris dans cette enceinte, ce ne fut que peu à peu qu'on parvint à acquérir les terrains qui forment actuellement la nouvelle ménagerie; plusieurs de ceux qui devaient y être réunis appartiennent encore à des particuliers, ce qui en rend le plan d'une irrégularité choquante, et s'oppose à la construclion des parcs nécessaires pour loger les animaux qui arrivent tous les jours.

On sent que le malheur des temps, la pénurie des finances, la chute des assignats, la cessation du commerce étranger, l'emploi de tous les fonds et de toutes les industries pour la guerre, durent mettre de grandes entraves à l'exécution des projèts qu'on avait formés.

En effet, non-seulement pendant les premières années de destruction, mais depuis $179^{5}$ jusqu'à la fin du siècle, l'établissement, constitué sur de nouvelles bases, se trouva dans la situation la plus extraordinaire, et présenta les contrastes les plus étonnans. On réunit au Jardin des maisons et des terrains de la plus grande importance, on acquit 


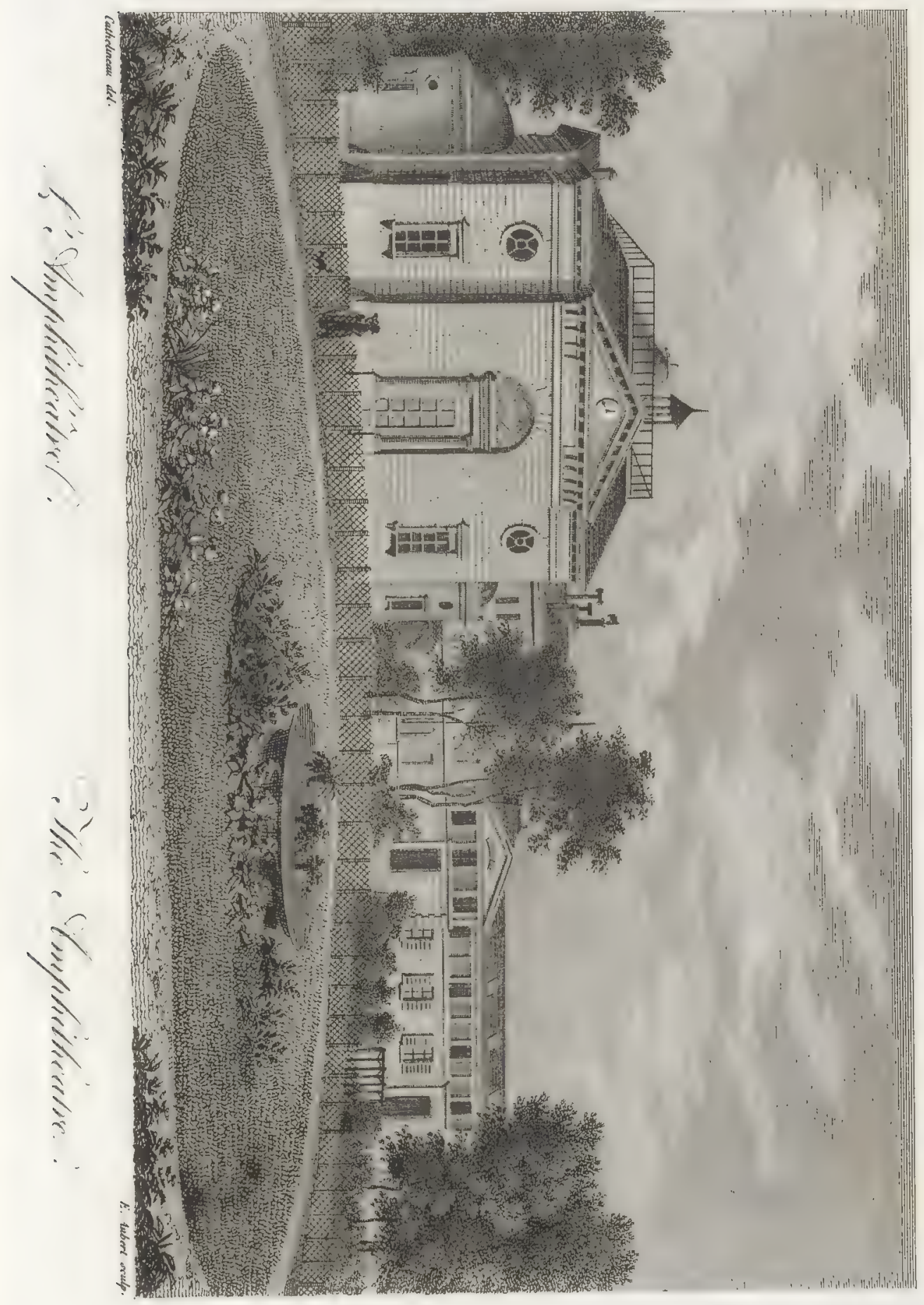


des collections magnifiques, on commença les plus utiles constructions; et cependant tout languissait dans l'intérieur; on entreprenait plusieurs choses à la fois, et rien ne pouvait être terminé; on manquait de fonds pour payer les ouvriers, de fourrages pour nourrir les animaux, d'armoires pour renfermer les objets les plus précieux; on cullivait des pommes de terre dans les carrés destinés aux plantes les plus rares, et l'établissement fut plusieurs fois menacé d'une décadence d'autant plus irréparable qu'elle portait à la fois sur toutes les parties : un obstacle était-il surmonté, d'autres renaissaient, et les fonds qu'on recevait étant employés à l'objet qui avait pour le moment fixé l'attention, d'autres objets se détérioraient par l'interruption forcée des travaux. Cependant, aux époques où la détresse se fit le plus sentir, il n'y eut pas un moment de découragement parmi les administrateurs; ils calculaient les mesures les plus convenables aux circonstances; ils se faisaient respecter en donnant l'exemple du zèle, de la modération et du désintéressement. Quelques-uns des professeurs ayant été appelés à des fonctions qui. les mettaient en relation avec le gouvernement, ils employèrent leur influence et leurs soins à soutenir l'établissement auquel ils étaient plus particulièrement attachés.

A la fin de l'année r 794 , l'amphithéâtre fut ter- 
miné et mis dans l'état oùil est maintenant. C'était auparavant un bâtiment carré ; on y ajouta les trois pavillons et le laboratoire de chimie, et le 25 janvier $179^{5}$, on y fit l'ouverture de l'école normale.

Cette institution extraordinaire était fondée sur un plan chimérique. On avait imaginé que des hommes déjà avancés en âge pourraient, après avoir entendu quelques leçons des grands maîtres, sè trouver capables de répandre l'instruction dans les provinces : tous les esprits sages sentaient qu'une pareille idée ne pouvait se réaliser ; mais on entendit au Muséum les savans les plus célèbres de la France exposer les fondemens et la doctrine de toutes les sciences; et les élèves venus de tous les départemens, trouvèrent le moyen d'étudier en détail et méthodiquement les sciences naturelles; tandis que des savans du premier ordre leur exposaient des théories qu'ils ne pouvaient comprendre parce qu'ils n'y étaient pas préparés. Ce spectacle, dont il n'y avait jamais eu d'exemple, frappa les imaginations, et il fixa les yeux sur un établissement devenu le centre de tous ceux qui pouvaient être consacrés à l'étude de la nature.

Depuis 1794 , époque de son organisation actuelle, jusqu'à la fin du siècle, le Muséum fut, comme nous venons de le dire, exposé à de grands dangers; mais il reçut des agrandissemens et des 
richesses considérables, dont on tira ensuile tout le parti possible.

Nous avons ditqu'en 1788 le Jardin avait été prolongé du côté du nord jusqu'à la rue de Seine, par l'acquisition de l'hôtel de Magny; mais cet emplacement, dans lequel on avait construit l'amphithéâtre, était enclavé entre des possessions étrangères. A l'ouest, il était séparé de la colline nommée le labyrinthe, par des maisons et des jardins appartenans à un particulier nommé Léger; à l'est, il était borné par une-vaste propriété nationale, où se trouvait autrefois la régie des fiacres, et dont on avait fait un magasin de farines. Le premier de ces terrains fut acheté pour le Muséum, en conséquence d'un arrêté du comité des finances du 9 juin 1795 ; le second lui fut concédé par une loi du mois d'août de la même année, et l'on prit aussitôt des mesures pour les faire servir' aux besoins de l'établissement. Dans la partie ouest, on arrangea le bâtiment principal pour y placer les bureaux de l'administration, et pour y déposer les collections qui ne pouvaient plus entrer dans le grand cabinet; on réserva pour des logemens de professeurs, les maisons situées le long de la rue de Seine jusqu'à la rue Saint-Victor, qui étaient habitées avant la révolution par une communauté religieuse nommée les nouveaux convertis, et l'on joignit au labyrinthe les jardins quiles entomaient. 
Les bâtimens et les cours à l'est de l'amphithéâtre, entre le jardin des semis et la rue, furent destinés à la construction de la serre tempérée, et à celle du cabinet d'anatomie; et le plan de ces édifices, présenté le 25 novembre par M. Molinos architecte du Muséum, ayant été adopté par l'assemblée, on commença les travaux. Ces travaux allèrent lentement par les causes que nous avons exposées: la serre tempérée ne fut terminée que cinq ans après, et c'est seulement en I 8 I 7 que le cabinet d'anatomie a été construit tel que nous le voyons aujourd'hui.

On avait arrêté depuis long-temps que tous les terrains situés à l'est et au nord de la serre tempérée scraient réunis à la ménagerie ; mais il fallait les acheter, et cette dépense parut trop considérable. Cependanton acquit d'abord quelques chantiers, qui servirent pour former les premiers parcs, dans lesquels on plaça des animaux ruminans. Les deux éléphans, mâle et femelle, de la ménagerie du Stathouder, qu'on avait pris lors de la conquête de la Hollande en $179^{5}$, étant arrivés au mois d'avril I 798 , furent logés dans une écurie et une cour du bâtiment nouvellement acquis.

On continua de travailler aux galeries du deuxième étage du cabinet; on reçut beaucoup de glaces des dépôts nationaux; mais la difficulté de se procurer toutes celles dont on avait besoin, et 
TROISIÈME ÉPOQUE.

celle d'avoir des stores, empêchèrent qu'on ne pût y étaler les collections de zoologie.

On employa les fonds dont on pouvait disposer à la construction d'une nouvelle serre absolument nécessaire pour placer des végétaux vivans que le capitaine Baudin devait apporter de son voyage. Ce voyage en Amérique ayant été fait pour le Muséum et l'ayant singulièrement enrichi, il est à propos d'en dire un mot.

Au commencement de 1796 , le capitaine Baudin, qui revenait de l'île de la Trinité, informa l'administration du Muséum qu'il avait laissé dans cette île une riche collection d'histoire naturelle, et qu'il la lui offrirait si on voulait lui donner un vaisseau pour aller la chercher. L'administration ayant fait cette demande au ministre, elle fut accordée, à condition que le capitaine Baudin cmmènerait avec lui quatre naturalistes, savoir : MM. Maugé et Levilain pour la zoologie, M. le Dru pour la botanique, et Riedley jardinier du Muséum, homme d'un zèle et d'une activité infatigables.

Baudin partit du Havre le 3o septembre $179^{6}$; son vaisseau fit naufrage aux îles Canaries, et le gouvernement espagnol lui donna un autre bâtiment pour remplir sa mission. Il se dirigea donc sur l'île de la Trinité ; mais cette île étant au pouvoir des Anglais, il ne put y entrer, et il se rendit 
à Saint-Thomas. Là on lui donna un bâtiment plus considérable, et il alla à Porto-Rico. Son séjour dans ces deux îles fut d'environ un an; il entra dans le port de Fécamp le 12 juin $r 79^{8}$, et sa collection, envoyée par la Seine, arriva au $\mathrm{Mu}$ séum le 12 juillet.

Jamais on n'avait reçu à la fois un aussi grand nombre de végétaux et surtout d'arbres des Antilles : $i l$ y avait une centaine de caisses dont plusieurs renfermaient des individus de six et jusqu'à dix pieds de hauteur; et les plantes avaient été si bien soignées pendant la traversée, qu'elles étaient en pleine végétation, et qu'elles réussirent très-bien dans nos serres.

Le résultat du voyage ne se borna point à procurer au Jardin des plantes vivantes, il enrichit également les cabinets : nos herbiers furent accrus d'un grand nombre de plantes des Antilles, recueillies et desséchées avec soin par MM. le Bru et Riedley, qui n'avaient pas négligé d'indiquer le lieu où elles avaient été ramassées. Riedley avait fait de plus une collection de tous les bois de Saint-Thomas et de Porto-Rico, et il avait attaché à chaque échantillon un numéro qui renvoyait au rameau fleuri du même arbre conservé dans l'herbier; ce qui donna au professeur de botanique la facilité de les déterminer.

Les deux zoologistes rapportaient aussi des 
peaux de quadrupèdes, des oiseanx et des insectes. La nombreuse collection d'oiseaux faite par Maugé, était surtout très-intéressante, parce que la plupart des espèces manquaiènt au Muséum, et que tous les individus étaient parfaitement conservés.

Le succès de ce voyage en fit projeter un autre, dont nous parlerons bientôt, et qui eut des résultats encore plus avantageux.

En 1 798, les professeurs présentèrent un mémoire au gouvernement pour lui exposer les besoins du Muséum : ils disaient que les magnifiques collections qu'on avait reçues étaient encore dans des caisses, où elles étaient exposées à être détruites par les insectes, parce qu'on n'avait point de local pour les étaler, et qu'il était absolument impossible de les mettre en ordre. Ils se plaignaient aussi de ne plus avoir aucun moyen de nourrir les animaux; parce que les entrepreneurs qui n'étaient pas payés de leurs avances, refusaient de faire de nouvelles fournitures. La même détresse continua en 1799 .

Cette situation était d'autant plus déplorable, que le Muséum avait reçu depuis sa nouvelle organisation les plus grandes richesses. Nous ne nous arrêterons pas à en faire ici l'énumération; nous nous bornerons à indiquer les acquisitions les plus importantes. 
Au mois de juin $\mathbf{r} 79^{5}$, on reçut le cabinet du Stathouder, riche dans toutes les branches d'histoire naturelle et surtout en zoologie.

En février 1796, M. Desfontaines fit don au Muséum de sa collection d'insectes de Barbarie.

En novembre même année, on reçut une collection de la Belgique.

Le même mois, l'académie des sciences donna au Muséum une pépite d'or du poids de vingtquatre marcs quatre onces, et le gouvernement lui fit remettre une collection de pierres précieuses qui était à l'Hôtel des monnaies. Au mois de février I 797 , le ministre acquit pour le Muséum la collection d'oiseaux que M. Levaillant avait faite en Afrique, et qui sert de type à son célèbre ouvrage; en 1798, celle que Brocheton s'était procurée à la Guiane; enfin la même année le capitaine Baudin fit arriver dans les serres et dans les galeries du Muséum, les nombreuses richesses de botanique et de zoologie que ses infatigables collaborateurs avaient recueillies sous les tropiques.

Le gouvernement n'avait cessé de prendre le plus grand intérêt à l'établissement : il avait fait pour lui tout ce qu'il pouvait faire; mais la pénurie des finances ne lui laissait pas la possibilité de fournir des fonds pour les dépenses qu'exigeait le placement des objets, l'entretien des bâtimens, 
le paiement des employés, la nourriture des animaux de la ménagerie. Les réclamations devenaient inutiles. Les fonds étaient absorbés par des armées qui conservaient leur courage, mais qui étaient accablées par le malheur; et l'état d'impuissance auquel on était réduit se manifestait également au dedans et au dehors. Les grands événemens qui eurent lieu en novembre r 799 , en déplaçant et concentrant le pouvoir, établirent un autre ordre de choses : le chef du nouveau gouvernement se rendit peu à peu tout-à-fait absolu; mais des conquêtes étonnantes couvrirent nos armées de gloire, et nous donnèrent tout à coup de grandes ressources.

L'embarras se fit encore sentir pendant les premiers mois de 1800 ; à cette époque même, on avait si peu de moyens, qu'on fut obligé d'autoriser M. Delaunay, qui était chargé de la surveillance de la ménagerie, à faire tuer les animaux les moins utiles, pour fournir à la nourriture des autres. Mais bientôt tout changea de face.

L'homme extraordinaire qui s'était placé à la tête de l'état sentait que sa puissance ne pouvait se soutenir uniquement par les conquêtes qui l'avaient établie; qu'après s'être rendu redoutable au dehors, il fallait se faire admirer dans l'intérieur en favorisant le progrès deslumières, en encourageant les arts et les sciences, en fondant des 
monumens qui, pendant la paix, assurent la gloire et. la prospérité des nations. Il tourna ses regards vers le Musćum, où tout se trouvait déjà préparé par les acquisitions qu'on avait faites; il lui fournit: des fonds pour continuer les travaux qu'on avait entrepris, pour acquérir une portion des terrains nécessaires à son agrandissement, et pour enrichir les collections.

Depuis quelques années la ménagerie n’avait point recu d'animaux étrangers, et si l'on excepte les lions qui avaient fait des petits, et les éléphans venus de Hollande, il y en avait très-peu de remarquables. On apprit qu'il s'en trouvait plusieurs. à Londres, et que M. Penbrock à qui ils appartenaient se proposait de les vendre: L'administration en ayant informé . M. Chaptal, alors ministre de l'intérieur, il consentit à en faire l'acquisition : M. Delaunay, sous-bibliothécaire, qui continuait à être chargé de la surveillance de la ménagerie, fut envoyé à Londres pour cet objet au mois de juillet $\mathbf{1} 800$, et il acheta, pour la somme de 17,500 francs, huit quadrupèdes, savoir : deux tigres mâle et femelle, deux lynx aussi mâle et femelle, un mandrille, un léopard, une panthère, une hyène et quelques oiseaux. Ces animaux arrivèrent sans accident, et ils furent placés dans les loges construites à l'extrémité de l'allée des marroniers. Sir Joseph Banks 
profita de: celte occasion pour envoyer au Muséum quelques plantes curieuses.

Dans le même temps, on plaça dans les galeries la girafe et d'autres animaux'rares, dont la préparation avait exigé beaucoup de soins; les squelettes, qui pendant long-temps avaient été réunis dans des salles où le public ne les voyait pas, furent mis en ordre; on examina les nombreux échantillons de minéraux qui se trouvaient dans les magasins ou dans des caisses', pour en retirer ceux qui devaient figurer dans le Cabinet, et la collection d'insectes fut classée par. M. Latreille, que le Muséum s'était attaché, en le nommant aide: naturaliste:en 1794. Chaque płofesseur ayant mis à part des échantillons, choisis parmi les doubles des objets qu'il avait déterminés, on en forma des collections classiques pour les écoles centrales des départemens. Enfin toutes les parties furent également soignées, parce que chacune d'elles était sous la surveillance d'un chef, et le mouvement progressif qui avait été imprimé ne se ralentit plus.

Cependant au mois d'octobre 1800 l'établissement courut le plus grand danger, et les administrateurs eurent lieu de craindre qu'il ne fût bientôt ruiné par une mesure que le ministre de l'intérieur, frère du premier consul, avait voulu étendre également à toutes les institutions pu- 
bliques : celle d'établir sous le titre d'administratcur comptable un directeur général ou intendant nommé par lui, qui serait seul chargé de l'administration générale et de la correspondance avec le gouvernement, et de réduire ainsi les administrateurs actuels du Muséum à la simple fonction de faire leurs cours et de conserver les collections dont le dépôt leur serait confié.

Les professeurs firent à ce sujet au ministre, les représentations les plus fortes. Ils lui prouvèrent que chaque partie avait besoin d'un chef, que l'administration était essentiellement liée à l'enseignement, que les intendans étaient toujours portés à faroriser telle ou telle partie, et qu'ils ne pouvaient connaître les détails d'un ensemble aussi vaste : que tous ceux qui avaient été à la tête de l'établissement, en exceptant Guy de la Brosse, Fagon et Dufay, l'avaient négligé, et que plusieurs même avaient arrêté les progrès qu'il devait naturellement faire : que M. de Buffon, qui seul depuis, avait mis sa gloire et son crédit à le rétablir, aurait lui-même senti que son étendue actuelle exigeait un autre régime : que M. Daubenton, l'ami et le coopérateur de Buffon avait refusé la place de directeur perpétuel que ses collègues lui avaient offerte par respect pour son âge et par reconnaissance pour les services qu'il avait rendus : que depuis la 
TROISIEME ÉPOQUE.

$9 j$

nouvelle organisation, l'ordre n'avait pas été troublé un instant, malgré les vicissitudes du gouvernement et les malheurs publics : que le $\mathbf{M u}$ séum étant immédiatement sous la dépendance et la protection du ministre, il suffisait d'un directeur annuel pour rendre compte de tout; qu'aucune dépense extraordinaire ne pouvait être faite que par l'ordre du ministre : que la place d'intendant donnée d'abord à un savant distingué, pourrait l'être bientôt à un homme qui n'aurait aucune idée de l'utilité des sciences naturelles : que les fonds destinés au Muséum pourraient être détournés pour d'autres objets selon les circonstances : que les professeurs se trouveraient dans un état de subordination qui affaiblirait leur zèle et paralyserait leurs efforts pour l'agrandissement du $\mathrm{Mu}$ séum : que quelques-uns d'entre eux qui avaient dans le gouvernement des places éminentes ne pourraient plus conserver leur chaire de professeur au Muséum, s'ils avaient au-dessus d'eux un chef perpétuel dont ils seraient obligés de recevoir les ordres. Le ministre ne se rendit point à ces raisons; il voulut nommer à la place de directeur M. de Jussicu; mais celui-ci ne profita de cette faveur que pour faire des représentations encore plus fortes, et pour empêcher l'exécution d'un plan qui aurait produit un mal irréparable. Heureusement rien n'était encore terminé, lors- 
qu'au mois de novembre, M. Chaptal ayant eu par intérim le portefeuille du ministère de l'intérieur, fit valoir auprès du premier consul les réclamations des professeurs.

On peut voir par les progrès que le Muséum a faits depuis, et par l'accord qui règne dans toutes les parties, combien l'administration qui le régit lui est convenable. Nous espérons que l'idée de centraliser une autorité qui n'a nulle relation avec les affaires politiques, ne se reproduira point sous le gouvernement éclairé et paternel qui a été rendu à la France. Lors de la fondation du Jardin du Roi, cet établissement était si peu de chose qu'un seul homme pouvait le régir et former le plan de son agrandissement; on n'y enseignait que la botanique, l'anatomie et la chimie, et même sous le point de vue médical; il fallait sans cesse obtenir pour lui des faveurs de la cour. Aujourd'hui les. dépenses du Muséum sont fixées par le budjet, et c'est aux divers administrateurs à combiner entre eux comment les fonds peuvent être le plus utilement employés. Chacun propose les améliorations possibles dans la partie dont il est spécialement chargé; tous se réunissent pour conserver l'harmonie de l'ensemble, pour justifier la confiance qui leur est accordée, et pour assurer la prospérité d'un établissement dont la gloire est pour eux une 
propriété commune. Lorsqu'un professeur succède à un autre, il peut présenter sous une forme différente la science qu'il enseigne; mais l'assemblée administrative est constamment animée du même esprit, et sa marche, plus ou moins active selon les circonstances, n'est jamais rétrograde, parce qu'elle est toujours dirigée vers le même but.

Les réflexions sur lesquelles nous nous sommes un moment arrêtés ne sont point étrangères à l'histoire du Muséum, et la suite du tableau que nous avons entrepris de tracer en démontrera la justesse.

En r8or, sous le ministère de M. Chaptal, à qui le Muséum a les plus grandes obligations, l'école de botanique, qui s'était prodigieusement enrichie depuis 1773 , fut agrandie d'un tiers, et les deux parterres qui sont vis-à-vis du cabinet furent plantés et arrangés comme on les voit aujourd'hui. La galerie supérieure du cabinet fut terminée et pourvue de glaces et de stores, et les principaux objets y furent arrangés méthodiquement. La serre tempérée fut achevée et garnie de magnifiques arbrisseaux. Le plan de la ménagerie étant définitivement arrêté, on acquit, pour la formation des premiers parcs, plusieurs des chantiers situés le long de la rue de Seine: on construisit dans la maison Léger de nou- 
velles salles pour un laboratoire de zoologie et pour des galeries de botanique : on plaça d'une manière provisoire dans plusieurs salles de la maison dite la liégie, ce qu'on avait de squelettes et de préparations anatomiques; et le Muséum se trouva dès 1802 organisé de manière que toutes les sciences naturelles pouraient y être également enseignées, que les diverses parties formaient un ensemble régulier, et que chacune d'elles était susceptible d'agrandissemens successifs. Tout prospérait: lestravaux se faisaient avec une activité surprenante; les cours attiraient les étranyers, et l'amphithéâtre était souvent rempli. Le plus grand ordre régnait partout. Les diverses secousses qu'on avait éprouvées depuis la nouvelle organisation n'avaient rien ébranlé; en contemplant les productions et les bienfaits de la nature, on reconnaissait qu'elle se montre toujours également grande et féconde après les orages.

Lors del'acquisition de la maison Léger en $179^{5}$, on avait arrêté qu'on placerait au rez-de-chaussée les bureaux de l'administration, qu'on établirait au premier étage un laboratoire de zoologie, et que le deuxième et le troisième étage seraient destinés pour la minéralogie et la botanique. Les distributions les plus nécessaires ayant été faites, la salle d'administration fut ouverte en $\mathrm{I} 800$, et les produits du règne végétal furent transportés de 
l'ancien cabinet dans la salle qui avait été préparée pour qu'on pût les y déposer.

Cet arrangement n'était que provisoire, il fallait profiter des pièces les plus vastes pour déballer les nombreux objets de zoologie et de minéralogie qui étaient dans des caisses, et se donner le: temps de construire des salles où l'on 'pût développer la collection de botanique. Il fut en conséquence décidé, sur la demande de M. Desfontaines, que le premier étage serait destiné à des galeries de botanique, que le laboratoire de zoologie serait transféré dans les pièces du second, d'où l'on avait retiré les minéraux, et que l'étage supérieur servirait de magasin. Les travaux intérieurs pour l'exécution de ce plan furent commencés en I 802 , mais ils ne purent être terminés que quelques années après.

C'est ici le lieu de parler de l'entreprise qui a le plus contribué à répandre au dehors la gloire du Muséum, et les connaissances dont il est le foyer. Nous ne pouvons le faire sans payer un tribut de reconnaissance à $\mathbf{M}$. de Fourcroy qui en forma le plan et qui en accéléra l'exécution. Lorsque ce savant vit que le Muséum était organisé d'une manière stable, il engagea ses collègues à se réunir et à publier en commun leurs observations, en s'attachant surtout à faire connaître les nourclles richesses renfermées dans les 
collections confiées à leurs soins. Ce projet ayant été adopté, on s'arrangea avec un libraire, et l'on convint de publier chaque mois un cahier de dix feuilles in- $4^{\circ}$, avec cinq ou six gravures exécutées par les meilleurs artistes sous la direction de M. Vanspaendonck. Comme il fallait s'entendre pour varier les sujets, les professeurs résolurent de se réunir une fois par semaine pour se communiquer réciproquement ce qu'ils se proposaient de publier, et ils chargèrent $\mathbf{M}$. Deleuze, l'un des aides naturalistes, de recueillir les mémoires et d'en surveiller l'impression. Le premier volume composé des six premiers cahiers fut terminé en 1802 , et l'ouvrage acquit tout à coup parmi les savans de l'Europe une réputation qui s'est toujours soutenue : la publication en fut ralentie lors de la cessation de tout commerce étranger; mais la rédaction en fut toujours également soignée. Il a eu le titre d' $A n$ nales du Muséum jusqu'au vingtième volume : il se continue sous le titre de Mémoires du Muséum. La collection complète forme aujourd'hui vingtsept volumes in- $4^{\circ}$. Les professeurs y ont admis des mémoires étrangers lorsqu'ils les ont jugés propres à faire connaître de nouveaux objets.

Quoique M. Daubenton se fût pendant quarante ans donné des soins pour réunir au cabinet du Roi les minéraux les plus utiles pour l'étude, et 
que depuis la nouvelle organisation on en eût reçu beaucoup des pays étrangers, la collection. était bien incomplète et même inférieure à celles de quelques amateurs. Elle l'aurait été pendant long-temps encore, si l'on n'eût profité d'une occasion qui se présenta pour la rendre tout à coup digne d'un établissement consacré à l'instruction publique.

Un Allemand nommé $\mathbf{W}$ eiss avait apporté à $\mathbf{P a -}$ ris un superbe cabinet de minéralogie qu'il voulait vendre tout ensemble sans en rien détacher. La vente étant annoncée, les professeurs du Muséum représentèrent au ministre, M. Chaptal, combien il était fâcheux de laisser sortir de France une réunion d'objets qu'il serait très-difficile de se procurer en détail, et ils lui demandèrent de les autoriser à traiter avec $M$. Weiss en lui offrant de lui céder, en échange de ses minéraux, des pierres précieuses, des morceaux de lapis-lazuli, et la pépite d'or que possédait le Muséum. Le ministre accueillit les observations des professeurs, et il demanda au conseil des mines un rapport sur le mérite de la collection. Elle se trouva composée de seize cent soixante-seize morceaux choisis, et fut évaluée ז50,00o francs. Après de longues discussions, M. Weiss accepta les arrangemens qu'on lui proposait; les pierres précieuses furent estimées par deux joailliers, l'un nommé 
par le ministre, l'autre par l'administration, et comme elles ne suffisaient pas pour compléter la somme convenue, le gouvernement paya l'excédant du prix. C'est de cette époque (en 1802) que le Muséum possède une suite régulière d'échantillons de minéraux, dans laquelle il se trouve seulement quelques lacunes qui se remplissent tous les jours.

La même année. I802, M. Geoffroy fit don au Muséum des objets qu'il avait recueillis en Égypte pendant un séjour de quatre années. Cette collection était d'autant plus précieuse qu'il s'y trouvait plusieurs des animaux sacrés conservés depuis des milliers d'années dans les tombeaux de Thèbes et de Mermphis ( $\mathbf{r}$ ).

Peu de temps après l'organisation définitive du Muséum on avait présenté le plan d'urie vaste ménagerie dans laquelle des animaux de toutes les classes et de tous les climats pourraient être placés d'une manière analogue à leurs habitudes. Ce projet conçu à une époque où l'on ne calculait pas les moyens d'exécution fut bientôt abandonné. Fn I 802, plusieurs terrains ayant été acquis pour placer les animaux paisibles, M. Molinos présenta un nouveau plan pour le logement des animaux féroces; c'est celui de la grande rotonde qui est au centre de la ménagerie. La première pierre

(1) Voyez le Rapport sur cette collection. Ann, du Mus, t. i, p. 23/. 
en fut posée en 1804 , et l'on continua d'y travailler pendant deux ans, et jusqu'à ce que les murs extéricurs fussent élevés à quelques pieds au-dessus du terrain. On reconnut alors que cet édifice n'était pas convenable pour le but auquel il était destiné, et le travail fut interrompu. Nous verrons bientôt comment on a profité de cette construction.

En 1804 , on plaça dans la galerie du second étage du cabinet un meuble qui en occupe toute l'étendue, au-dessus duquel sont arrangés, dans des cadres, les insectes, les coquillages, les crustacés, les madrépores, et dont la base est formée de tiroirs dans lesquels sont renfermés tous les doubles de la collection d'entomologie et quelques insectes uniques qu'on n'ose exposer à la lumière qui pourrait altérer leurs couleurs.

Vers la même époque, le Muséum fut enrichi des collections les plus précieuses pour la géologie. L'empereur Napoléon lui donna celle des poissons fossiles qu'il avait acquise de M. le comte de Gazola, celle du même genre que la ville de. Vérone lui avait offerte, et celle des roches de Corse qu'il avait reçue de M. de Barral, officier dans cette île. Ces objets remplissent aujourd'hui une des salles du cabinet.

Pendant ce temps, les travaux du laboratoire de zoologie se faisaient avec une telle activité, 
que dans l'année i 805 on prépara et monta cent un quadrupèdes, cinq cents oiseaux, autant de reptiles et de poissons.

L'année précédente, l'éléphant mâle, l'un des deux qu'on avait amenés de Hollande, étant mort, M. Cuvier entreprit d'en faire la dissection. Ses élèves en zoologie et en anatomie, et le peintre, M. Maréchal, l'ayant secondé dans ses recherches, il rassembla les descriptions et les dessins nécessaires pour faire connaître les organes particuliers à cet énorme quadrupède, et ceux qui ont chez lui un développement plus considérable qu'il ne l'est dans les autres animaux. Ce travail était cependant encore imparfait, et il restait bien des choses à examiner, lorsque dixhuit mois après, au mois de juillet $\mathbf{1} 804$, un autre éléphant, qu'on avait acheté pour remplacer le premier, périt de même. Ni l'excessive chaleur, ni le défaut d'un local convenable ne purent ralentir le zèle de M. Cuvier; il examina et fit dessiner tout ce qu'il n'avait pu bien voir la première fois; et quelques années après l'éléphant femelle étant mort, il eut l'occasion de vérifier ses observations précédentes, et de comparer les deux sexes. Il est résulté de ce travail que l'anatomie de l'éléphant, dont on n'avait auparavant que le squelette, est aujourd'hui aussi bien connue que celle du cheval; ce qui prouve l'utilité des 
ménageries pour les progrès de l'histoire naturelle.

Ce qu'on eut à souffrir pour disséquer l'éléphant dans la saison la plus chaude de l'année fit sentir la nécessité d'avoir un laboratoire d'anatomie, où les objets pussent être commodément préparés avec les précautions convenables, et l'on construisit celui qui existe maintenant.

Cette mème année 1804 , le Muséum se trouva tout à coup enrichi de la collection la plus considérable qui lui fût jamais parrenue pour la zoologie et la botanique. C'est ici le lieu d'en rappeler l'origine.

Au commencement de l'année I 800 , l'Institut de France proposa au premier consul d'envoyer deux vaisseaux aux Terres australes pour y faire des découvertes sur la géographie et sur les sciences naturelles et physiques. Le premier consul adopta cette idée, et sur la présentation de l'Institut et du Muséum d'histoire naturelle, il nomma pour faire partie de l'expédition, vingttrois hommes instruits, qui furent chargés de s'occuper uniquement de ce qui est relatif au progrès des sciences. Les deux vaisseaux le Géographe et le Naturaliste, commandés le premier par le capitaine Baudin, le second par le capitaine Hamelin, partirent du Havre le r9 octobre I 800 ; ils relâchèrent à l'Ile-de-France où restè- 
rent la plupart de ceux qui s'étaient embarqués pour des recherches scientifiques.

Après avoir quitté l'lle-de-France, les deux vaisseaux allèrent reconnaître la côte occidentale de la Nouvelle-Hollande, et ils se rendirent à Timor où ils passèrent six semaines. De là ils retournèrent visiter la même côte, ils firent le tour de la terre de Diemen; et remontant au nord, ils allèrent au port Jackson où ils firent un séjour de cinq mois. Ils reprirent ensuite la route de Timor en passant par le détroit de Bass. De Timor ils revinrent en France, et ils entrèrent dans le port de Lorient le 25 mars i 804 .

Des cinq zoologistes qui avaient été nommés pour cette expédition, deux s'étaient arrêtés à l'Ile-de-France. Les deux autres, Maugé et Levilain, étaient morts pendant le voyage.Péron, resté seul, se lia de la plus intime amitié avec M. Lesueur, peintre d'histoire naturelle et très-bon observateur; ces deux hommes infatigables vinrent à bout de recueillir, de conserver et de décrire une infinité d'objets. On passa quinze jours à débarquer la collection au port de Lorient, et elle fut de suite envoyée au Muséum. Pour en donner une idée, nous ne saurions mieux faire que de transcrire ici quelques phrases du rapport fait à l'Institut par M. Cuvier.

"Chaque jour dévoile mieux, dit-il, l'importance 
TROISIÈME ÉPOQUE.

et l'étendue de cette collection de zoologie. Plus de cent mille échantillons d'animaux grands et petits et appartenant à toutes les classes, la composent. Elle a déjà fourni plusieurs genres importans; et le nombre des espèces nouvelles, d'après le rapport des professeurs du Muséum, s'élève à plus de deux mille cinq cents.

" Tout ce qu'il était possible de conserver, ils l'ont rapporté soit dans l'alcohol, soit empaillé avec soin, soit desséché. Lorsqu'ils ont pu préparer des squelettes ils ne l'ont pas négligé, et celui du crocodile des Moluques prouve jusqu'où leur zèle s'est étendu à cet égard."

Le même voyage nous procura plusieurs animaux vivans, du nombre desquels étaient le zèbre et le gnou que M. Jansen, gouverneur du Cap, envoyait à l'impératrice Joséphine, et qu’elle donna au Muséum.

La collection de botanique n'était pas moins importante que celle de zoologie. La végétation de la Nouvelle-Hollande ne ressemble point à celle des autres parties du globe. Quelques plantes nous étaient déjà connues par les Anglais et par le voyage de $M$. de la Billardière, mais elles étaient en petit nombre auprès de celles qui nous furent apportées en r 804 . Il y avait plusieurs caisses d'arbrisseaux vivans qui se sont facilement multipliés, un très-grand nombre de graines qui ont levé, et 
des herbiers très-bien conservés, dans lesquels les trois quarts au moins des plantes étaient nouvelles, et dont plusieurs même ne sont pas encore connues, malgré les savantes recherches de M. Robert Brown. Quelques-unes ont été publiées dans nos Annales. Ce qu'il faut surtout remarquer, c'est que les plantes de la Nouvelle-Hollande, depuis le port Jackson jusqu'au détroit d'Entrecasteaux, ne sont point de serre chaude comme celles des tropiques; toutes peuvent passer l'hiver en pleine terre dans les départemens méridionaux de la France, et un grand nombre ne craindraient point les hivers à Paris. Aussi, depuis que nous avons reçu cet envoi, a-t-on vu s'introduire dans les jardins les métrosidéros, les mélaleuca, les leptospernum, qui par la beauté de leurs fleurs, ont d'abord excité l'admiration. Les magnifiques eucalyptus qui dans leur pays natal s'élèvent à cent cinquante pieds, et dont le tronc acquiert sept à huit pieds de diamètre, commencent à se multiplier. On les conserve encore dans l'orangerie à cause de l'époque à laquelle ils fleurissent. Mais en lesélevant de graine, on parviendra à changer leurs habitudes, et ils seront cultivés dans nos parcs. C'est du Muséum que de beaux individus de tous ces arbres de la famille des myrtes, se sont répandus chez les pépiniéristes, et de là dans toute la France. 
Tandis qu'on bâtissait de nouvelles salles pour le cabinet, et qu'on terminait la serre tempérée, on ne négligea point les travaux de la ménagerie. Chaque année on avait acquis quelques arpens de terrain le long de la rue de Seine, et on avait construit successivement des parcs et des fabriques variées pour les cerfs, les daims, les axis, les bouquetins, les mérinos, le gnou, les kanguroos, le zèbre, etc. Mais il manquait deux choses très-essentielles, $\mathrm{I}^{\circ}$ un local particulier pour les singes, dont on reçoit fréquemment des espèces peu connues, qu'il faut placer les unes auprès des autres pour qu'on puisse les observer et les comparer ; $2^{0}$ une volière.

I.es oiseaux aquatiques tels que les cygnes, les canards, le pélican, etc., se trouvaient fort bien dans les bassins; les paons se promenaient dans la ménagerie ou dans l'enclos gui est au centre du Jardin et creusé jusqu'au niveau de la rivière ; les autruches et les cazoars avaient un enclos particulier; mais les oiseaux de proie, et tous ceux qu'on est obligé d'enfermer dans des cages, étaient dispersés.

Comme les fonds extraordinaires accordés au Muséum pour des constructions avaient été absorbés par les travaux du cabinet et de la serre tempérée, on ne pouvait construire à neuf un édifice tel qu'on le désirait: on se détermina donc 
à arranger, aussi bien que le local le permettait, un bâtiment situé sur la rue de Seine, vers le milieu de la ménagerie. A l'aide de ce bâtiment et des cours adjacentes, on pratiqua pour les singes, à l'exposition du couchant, des loges disposées sur une même ligne et fermées par un grillage et des portes vitrées. On plaça à la suite, et le long du même mur, de grandes cages pour les oiseaux de proie, et en retour du côté du sud, on arrangea un abri pour les faisans etautres oiseaux de bassecour. Cette construction, qui existe encore, doit toujours être considérée comme provisoire : elle n'est point en accord avec les autres parties de la ménagerie; elle n'est point à une exposition favorable; elle n'a pas même l'étendue nécessaire pour qu'on puisse y placer à côté les uns des autres les animaux d'une même famille. On se propose de profiter d'un chantier, qui vient d'être réuni au Musétum, pour la remplacer par deux fabriques, l'une pour les singes et les quadrupèdes d'une petite taille, l'autre pour une volière.

En I 805 , époque à laquelle fut terminée la construction dont nous venons de parler, la ménagerie, quoiqu'elle n'offrît point assez d'espace, et qu'elle ne fût pas décorée des bâtimens pittoresques et des arbres superbes qu'on y voit aujourd'hui, présentait un spectacle très-propre à exciter l'intérêt des naturalistes ct à satisfaire la curiosité 
du public. Mais quand même on aurait pu exécuter les plans formés pour l'agrandir, pour la peupler et pour loger convenablement tous les animaux, elle n'aurait pas eu sur les progrès de la zoologie l'influence qu'on en doit attendre, si on ne l'cût organisée de manière à la mettre en rapport avec les diverses branches de cette science. C'était là ce qu'on s'était proposé en la plaçant dans les attributions et sous la direction immédiate du professeur chargé d'enseigner l'histoire des quadrupèdes et des oiseaux, et M. Geoffroy ne négligeait rien pour parvenir à ce but. Mais, obligé de faire ses cours, d'entretenir des correspondances, de classer, de nommer et de compléter la collection zoologique du cabinet, il lui était impossible de suivre tous les jours le mouvement de la ménagerie. Il demanda, en conséquence, qu'on lui donnât un adjoint qui pût s'en occuper continuellement, et qui, par ses connaissances, fût en état de le suppléer. L'administration duMuséum ayant reconnu l'importance d'une telle place, y nomma, le 2 I décembre ı 805 , avec le titre de garde de la ménagerie, M. Frédéric Cuvier, frère du professeur, et connu par d'excellens mémoires imprimés dans les Annales. Elle adopta en même temps le plan proposé par M. Greoffroy, pour en faire un établissement d'une utilité toujours croissante, et dans lequel toutes les observations qui se présen- 
tent sont liées aux observations antérieures et à celles qu'on pourra faire dans la suite. Ce plan, dont la ménagerie du Muséum offre le premier exemple, a été réalisé et coordonné avec les changemens et les auymentations qui ont eu lieu depuis, et chaque jour on en obtient les plus heureux résultats. L'ordre établi dans l'ensemble et la surveillance des détails en. ont rendu l'exécution facile. Les animaux sont placés et soignés d'après ce qu'on sait de leur nature et de leurs besoins. Le garde décrit ceux qui ne sont pas bien connus, et fait dessiner ceux dont on n'a pas encore une figure exacte; il observe chez tous ce qui est relatif à l'instinct, aux habitudes, à l'accouplement, à la gestation, à la manière de nourrir les petits, etc. ; il compare chaque animal qui arrive avec les espèces analogues, et il fait demander dans les pays étrangers ceux qu'on désire se procurer pour les mieux connaître, et ceux dont on croit devoir tenter la naturalisation. Un animal vient-il à mourir, il est à l'instant porté au laboratoire d'anatomie; là on le dépouille pour envoyer la peau au laboratoire de zoologie, où le professeur la fait monter si l'animal n'est point dans le cabinet. On prépare ensuite le squelette, si on ne l'a pas déjà, et l'on met dans l'esprit-devin toutes les parties molles qu'on croit devoir conserver : on ne néglige pas même de rechercher 
s'il n'y aurait pas dans le corps de l'animal quelques vers intestinaux, qui pourraient donner lieu à de nouvelles observations.

Ainsi, c'est par la ménagerie que le cabinet de zoologie, celui d'anatomie comparée, et la collection des vélins s'enrichissent tous les jours ; c'est par elle qu'on peut étudier la nature vivante, reconnaître l'influence de l'éducation et de la domesticité sur les animaux sauvages, améliorer les races, propager les espèces utiles; et les frais qu'il en coûte pour l'entretenir, ne sont rien auprès des avantages qu'on en retire.

En 1806, les galeries d'anatomie comparée ayant été provisoirement disposées, aussi bien que le permettait le local, elles furent ouvertes au public. On fut étonné d'y voir tout à coup arrangés méthodiquement, non-seulement les squelettes des animaux qu'on avait pu se procurer, mais encore la série de tous les organes, préparés de manière à pouvoir être étudiés et comparés dans les diverses classes d'animaux: toutes ces préparations avaient été faites par M. Cuvier ou sous sa direction.

Pendant que ce savant s'occupait à former un cabinet d'anatomie comparée, il reconnut que la plupart des ossemens fossiles n'ont point leur analogue parmi les êtres vivans; et voulant pousser ses recherches sur cet objet plus loin qu'on ne 
I 4 HISTOTRE DU MUSÉUM.

l'eût jamais fait, il ne négligea rien pour en réunir un grand nombre. Il en trouva de trèsremarquables dans les carrières de Montmartre, d'autres lui furent envoyés d'Allemagne et de diverses contrées; et il publia dans nos Annales une série de mémoires où il fait connaître plusieurs espèces de quadrupèdes qui vivaient sur notre globe avant la dernière révolution qui en a changé la surface. Ces animaux sont bien antérieurs à ceux qu'on trouve parmi les momies d'Égypte. Ils appartiennent à diverses époques, et diffèrent d'autant plus des animaux actuels que ces époques sont plus reculées.

Après cette publication, M. Cuvier donna au Muséum la collection qu'il avait rassemblée, et quoiqu'elle fût d'un très-grand prix parce qu'elle était unique en son genre, il ne voulut recevoir en échange que des doubles des livres d'histoire naturelle qui étaient à la bibliothèque. Cette collection réunie à celle des poissons du mont Bolca, remplit aujourd'hui une des salles du cabinet.

Nous avons dit qu'en 1802 , l'assemblée des professeurs avait arrêté que le premier étage de la maison Léger serait arrangé de manière à ce qu'on pût y développer les collections végétales. Les travaux nécessaires pour la disposition du local ne furent terminés qu'en r 807 . Pendant cet intervalle, le professeur de botanique avait exa- 
miné et réuni ce qu’on possédait, et en i 808 tout fut placé comme on le voit actuellement.

Autrefois les herbiers étaient renfermés dans des caisses et des portefeuilles, et la collection des fruits, des graines, des gommes, des résines, etc., était dans des bocaux avec l'ancien droguier de Geoffroy. Ise tout se trouvait dans la petite salle du cabinet où sont aujourd'hui les poissons. M. de la Marck qui, avant la nouvelle organisation de l'établissement, était chargé du soin des herbiers, avait plusieurs fois proposé de les mettre en ordre; mais il fallait un local pour les placer, et c'est ce qu'on n'avait encore pu obtenir. Les divers herbiers étaient séparés, ils n'étaient point classés, on n'avait même pu les réunir tous dans la salle du cabinet. Ceux de Commerson avaient été mis en dépôt chez M. de Jussieu, celui de Dombey avait été prêté par un ordre du ministre à M. l'Héritier qui s'était chargé de le décrire; la collection était inutile pour l'étude, parce qu'on ne savait où trouver ce qu'on cherchait.

Les nouvelles galeries de botanique ayant été terminées, une grande salle garnie de cases fut consacrée aux herbiers que M. Desfontaines réunit en arrangeant les plantes dans l'ordre des familles naturelles, et en ayant soin de marquer de quel herbier particulier chaque échanlillon avait été liré. On mit à part les herbiers 
qui, comme celui de Tournefort, servent de type à un ouvrage classique, et tout fut disposé de manière qu'on est sûr de mettre la main sur le genre qu'on veut examiner, et que s'il y a quelques lacunes, on peut les remplir soit par de nouvelles recherches, soit en se procurant par voie d'échange ce qu'on n'a point encore. Une seconde salle fut destinée pour les fruits et autres produits du règne végétal, une troisième pour les échantillons de bois; si bien qu'on peut comparer toutes les parties des végétaux, et que tout ce qu'on recoit de nouveau peut à l'instant être mis à sa place.

L'ouverture des galeries de botanique fait époque pour le progrès de la science. Le public n'y est point admis indistinctement; mais ceux qui veulent s'instruire reçoivent de la part du professeur toutes les communications dont ils ont besoin. La collection étant la plus nombreuse et la plus complète qui existe, c'est là qu'on peut comparer les espèces nouvelles et celles qui sont connues, et s'assurer de la nomenclature. Les savans qui ont publić des monographies, comme M. de la Roche et M. Dunal, ou l'histoire des plantes d'un pays, comme MM. de Humboldt, Bonpland et Kunth ; ou des histoires générales, comme M. de Candolle , n'auraient jamais pu donner à leur travail la même exactitude ni la même étendue, s'ils n'a- 
vaient eu la facilité de consulter les herbiers du Muséum.

L'utilité de ce dépôt estsi généralement reconnue par les botanistes, que plusieurs d'entre eux s'empressent d'y réunir les plantes qu'ils ont découvertes et décrites. Nous devons à la générosité de M. le baron de Humboldt le don le plus considérable qui nous ait été fait en ce genre, celui de l'herbier qu'il a rapporté de son voyage dans les régions équinoxiales de l'Amérique, ct qui se compose de 4,6oo espèces dont plus de 3,ooo étaient inconnues avant lui. Cet herbier est d'autant plus précieux qu'il renferme tous les échantillons d'après lesquels ontété faites les gravures qui accompagnent l'Histoire des plantes équinoxiales qu'il a publiée de concert avec soncompagnon de voyage M. Bonpland, et son savant collaborateur M. Kunth.

La formation de deux cabinets particuliers pour la botanique et l'anatomic, avait permis de consacrer l'ancien cabinet à la zoologie et à la minéralogic ; mais le local se trouvait encore trop resserré, et l'édifice, dont le nom même indiquait qu'il devait d'abord fixer l'attention, ne répondait plus à la beauté des constructions nouvelles. Le gouvernement, frappé de cette considération, résolut de l'agrandir autant que le réclamait le besoin de la science, et de le rendre digne de la célébrité de l'établissement. Pour faire sentir l'im- 
portance de ce service, et pour faire connaitre l'histoire d'un monument qui est aujourd'hui le plus beau qui existe en ce genre, il ne sera pas inutile d'entrer dans quelques détails et de reprendre les choses de plus haut.

Nous avons vu qu'en 1766 le cabinet était borné à deux salles, et que Buffon en doubla l'étendue en cédant son logement, et faisant construire l'escalier à côté de la grande porte du Jardin, situéc vis-à-vis de l'allée des tilleuls. Après la nouvelle organisation, on arrêta de construire au second étage une galerie éclairée par le haut. Les travaux pour l'exécution de ce plan, commencés en 1794 et souvent interrompus, furent terminés en I 80 I, et le second étage se trouva offrir deux fois autant de place que le premier, parce qu'il n'y avait point de croisées et que le dessus de la bibliothèque donnait une salle de plus. Ce cabinet fut d'abord magnifiquement meublé, et parut suffisant ; mais, depuis $\mathrm{I} 80 \mathrm{r}$, on avait reçu un si grand nombre d'objets, et la ménagerie avait fait arriver successivement tant de quadrupèdes qu'on reconnut la nécessité de l'agrandir de nouveau. Le gouvernement voulant que cette entreprise fût exécutée dans son ensemble, demanda à la fin de décembre 1807 , l'avis des professeurs, qui présentèrent à M. Crétet, alors ministre de l'intérieur, un plan dressé par M. Molinos. Ce plan fut adopté, 
et les fonds nécessaires ayant été assignés, on commença les travaux en 1808 . On supprima l'escalier pour ajouter sansinterruption trois nouvellessalles à la suite de celles qui cxistaient au premier élage, et l'on prolongea la galerie du second jusqu'à la terrasse élevée au-dessus de la rue derrière la butte plantée d'arbres verts. La principale porte et le grand escalier de l'édifice furent placésà l'extrémité; et l'entrée du Jardin sur la rue fut ouverte entre la bibliothèque et la maison ancienneiment nommée l'intendance.

Ces travaux très-considérables ayant été terminés en r8 ro, sous le ministère de M. de Montalivet, les dispositions intérieures furent faites avec tant de célérité, qu'au mois de mars i 8 I I on put arranger les collections. Des trois nouvelles salles l'une fut destinée pour les roches, les deux autres pour les produits volcaniques, et pour la belle collection de fossiles dont une partie avait été cédée au Muséum par M. Cuvier.

Au second étage, le prolongement de la galerie et trois salles dont la dernière a une porte sur la terrasse, furent employés à développer la collection des quadrupèdes et des singes, et à placer ceux qui étaient dans les laboratoires, ou conservés dans des caisses, parce qu'on n'avait pu les faire entrer dans les armoires du cabinet.

Lorsque les travaux de construction des gale- 
ries furent achevés, on reprit ceux de la grande rotonde située au milieu de la ménagerie, qui avaient été suspendus pendant quatre ans. On se conforma au premier plan pour l'extéricur, mais on en modifia la distribution intérieure de manière à pouvoir y placer les animaux herbivores, qui, comme l'éléphant, les chameaux, etc. , ont besoin d'être chauffés et soignés pendant l'hiver, et lorsque les femelles allaitent leurs petits.

L'édifice fut achevé en I 8 ז 2 . Il forme dans la ménagerie une décoration pittoresque, mais on a reconnu qu'il n'était pas très-propre à l'usage auquel il est employé. Comme il est isolé, et composé de cinq pavillons qui ont chacun une porte ouverte sur les parcs, il est impossible d'y entretenir une chaleur égale pendant la mauvaise saison, et les animaux, quelques précautions qu'on prenne, y sont exposés à des courans d'air toujours dangereux. Il serait à désirer qu'on construisît, pour les animaux herbivores des pays chauds, un bâtiment dans le genre de celui qu'on a fait pour les animaux féroces, composé d'une suite de pièces exposées au midi, où les animaux seraient commodément logés et à l'abri des rigueurs de la saison. Si le gouvernement jugeait i propos de faire cette dépense qui contribuerait ì la prospérité de l'élablissement, à la conservalion et à la propagalion des animaux qu'on se pro- 
cure à grands frais, la rotonde offrirait l'édifice le plus convenable pour une bibliothèque, soit par sa situation au centre et dans le lieu le plus agréable du Jardin, soit par sa distribution intérieure, qui serait très-commode pour le classement et l'arrangement des livres. La bibliothèque actuclle ne saurait rester long-temps où elle est. Le local est beaucoup trop resserré; les livres y sont sur plusieurs rangs, les tablettes ne suffisant pas pour les contenir tous. La salle qu'elle occupe est d'aillcurs absolument nécessaire pour l'agrandissement du cabinet. Elle se trouve à la suite de la galerie des poissons et des reptiles dont la collection, la plus riche qu'on ait jamais eue, doit être développée pour que les objets y soient arrangés méthodiquement et de manière qu'on puisse les voir et les étudier.

Nous avons parlé des diverses constructions qui furentfaites depuis I 805 jusqu'à I 812 , elles étaient d'autant plus nécessaires que, pendant tout ce temps, il arriva chaque année de nouvelles richesses.La collection de roches de Corse de M. Rampasse fut acquise et donnée au Muséum par l'empereur pour faire suite à celle de M. de Barral : en I 808, M. Geoffroy rapporta de Lisbonne une trèsbelle collection dans toutes les parties de l'histoire naturelle; cn r809, le ministre acheta pour le Muséum les échantillons de tous les bois de l'Amé- 
rique septentrionale, recueillis et rapportés par M. Michaux fils qui a publié l'histoire des arbres de cette contrée; ainsi que l'herbier du même pays, qui est le type de l'ouvrage de M. André Michaux père, mort à Madagascar. En 18 ro, on reçut vingtquatre animaux de la ménagerie du roi de Hollande.

On reçut aussi des minéraux envoyés d'Italie et d'Allemagne par M. Marcel de Serres; des animaux offerts en présent, de beaux herbiers faits à Cayenne par M. Martin qui y dirigeait les pépinières, et qui a introduit dans cette colonie la culture de l'arbre à pain en propageant de bouture un individu qui nous avait été apporté des îles des Amis par MM. la Billardière et la Haye, et que nous lui avions fait passer après l'avoir gardé un an dans nos serres (I).

L'état affreux dans lequel la France se trouvait en 18r3 ne permettant pas au gouvernement de s'occuper des établissemens destinés au progrès des sciences et des arts, les administrateurs du Muséum furent obligés de réduire toutes les dépenses, de s'interdire toute acquisition, et de renvoyeràune époque plus favorable les constructions qu'on avait entreprises ou projetées. La cor-

(1) M. Martin avait, plusieurs années auparavant, porté de l'Ile-deFrance à Cayenne, le poivrier, le muscadier et le giroflier qui y produisent aujourd'hui des récoltes abondantes. 
respondance avecles paysétrangers fut interrompue, et le nombre des élèves du Muséum diminua, parce que la plupart des jeunes gens étaient employés aux armées. Cependant les travaux essentiels continuèrent avec le même ordre, et l'on redoubla de soins pour conserver ce qu'on avait. acquis précédemment.

Lorsqu'en 1814 , les troupes étrangères entrèrent à Paris, un corps de Prussicns se présenta à la porte du Muséum où il se proposait de bivouaquer: le danger était imminent, et dans ce moment de trouble les professeurs n'avaient aucun moyen de parvenir jusqu'à l'autorité. Le commandant de la compagnie ne pouvait céder à leurs représentations et s'éloigner du poste qui lui avait été assigné ; il consentit cependant à attendre deux heures, et ce temps suffit pour nous mettre à l'abri de toute crainte. Un savant illustre dont le nom honore également et la Prusse sa patrie, et la France qu'jl a choisie pour y publier ses ouvrages, profita de la facilité qu'il avait d'arriver jusqu'au général prussien, et il en obtint une sauveyarde pour l'établissement. Le Muséum fut exempt de. tout logement militaire, et quoiqu'on n'en refusât l'entrée à personne, il n'y eut pas la moindre dégradation. L'empereur d'Autriche, celui de Russie, le roi de Prusse vinrent admirer les richesses qu'il renfermait, et prendre des renseignemens 
12 í

IISTOIRE DU MUSÉUM.

sur son organisation, afin de former chez eux des établissemens analogues.

En 1815 , le sort jaloux du bonheur de la France nous condamna à revoir une seconde fois entrer les étrangers avec des intentions plus hostiles, et nous eûmes tout lieu de craindre que le cabinet d'histoire naturelle ne fût dépouillé d'une grande partie de ses collections, et qu'on ne se fit rendre la plupart des objets qu'on y avait réunis à la suite des conquêtes, comme cela se pratiquait au Muséum des arts. En effet, la belle collection du cabinet du stathouder fut réclamée, et M. Brugmann fut envoyé à Paris pour la recevoir et la faire emballer. La mission dont il était chargé causa la plus vive inquiétude aux administrateurs du Muséum. Si tout ce qu'on avait reçu de Hollande eût été rendu, la collection qu'ils avaient arrangée se serait trouvée incomplète, elle n'aurait plus formé cette série qui en fait le principal mérite. M. Brugmann était trop éclairé pour ne pas sentir que la collection telle qu'elle se trouvait dans les galeries du Muséum de Paris, pouvait servir à l'instruction des savans étrangers comme à celle des nationaux, et que, lorsque les objets analogues seraient séparés, ils n'offriraient plus les mêmes moyens de comparaison; mais il était de son devoir de se conformer exactement aux ordres qui lui avaient été donnés; il pouvait seu- 
TROISIE ME ÉPOQUE.

123

lement se prêter à tous les moyens de conciliation, et plaider indirectement la cause du Muséum en défendant celle des sciences. Dans cette circonstance difficile, les professeurs s'adressèrent à M. le baron de Gagern, ministre plénipotentiaire de Hollande, qui seul pouvait suspendre l'exécution des mesures que M. Brugmann avait prises, et obtenir du souverain lui-même qu'il voulût bien révoquer ses ordres. La négociation eut un plein succès. Il fut convenu qu'on ferait pour la Hollande une collection équivalente à celle qu'on avait reçue, mais qui serait choisie parmi les doubles de celle du Muséum. Cette nouvelle collection, composée de dix-huit mille morceaux formant une série, était, au jugement de M. Brugmann lui-même, plus précieuse et plus alile pour les Pays-Bas que l'ancien cabinet du stathouder. Ainsi tout fut arrangé dans l'intérêt des sciences, et sans que de part ni d'autre on témoignât le moindre mécontentement. A la vérité, les armoires du cabinet se trouvèrent fort éclaircies, mais il n'y manqua aucun objet essentiel. Les vides qui y furent faits alors ont été remplis depuis, et souvent par des objets qu'on n'avait jamais possédés en France, et dont plusieurs sont absolument nouveaux.

Nous ne pouvons nous dispenser de témoigner ici notre reconnaissance à l'empereur d'Autriche 
qui vint plusieurs fois visiter l'élablissement, qui fit venir à Paris M. Booze, jardinier de Schœenbrunn, avec des plantes qui n'étaient point au Jardin du Roi, qui nous fit don de deux belles collections, l'une de vers intestinaux faite par M. Bremser, l'autre de champignons imités en cire avec la plus grande exactitude pour les formes et les couleurs, et qui chargea M. Schreiber d'envoyer à l'administration du Muséum un catalogue des échantillons doubles de son cabinet pour que les professeurs pussent choisir ce qui leur manquait, et donner d'autres objets en échange. Ces échanges furent également avantageux pour Vienne et pour Paris. On's'empressa de renvoyer au pape des pierres gemmes diversement travaillées qu'on avait reçues de Rome, et dont le prix n'était point relatif à la science.

Enfin, des objets d'histoire naturelle, et des livres pris chez des particuliers et envoyés au $\mathbf{M u}$ séum dans le temps de l'émigration, ayant été gardés comme en dépôt, ils ont été rendus aux anciens propriétaires, et les administrateurs ont toujours obtenu pour cela l'autorisation du ministre.

Aussitôt que la paix a été rétablie, le Roi a continué à favoriser l'agrandissement du Muséum. Les finances de l'état ayant été épuisées par les malheurs qu'on avait éprouvés, on n'a pu d'abord 
donner à cet établissement tous les secours dont il avait besoin. Comme il avait moins souffert que les autres, il y avait moins à réparer : au lieu de 300,000 fr. qui lui étaient accordés pour ses dépenses annuelles, il n'a été porté qu'à 275,000 fr. pendant les deux premières années; mais ensuite tout a été rétabli; et depuis i 8 I 8 , sous le ministère de $\mathbf{M}$. Laîné, il a reçu des fonds extraordinaires pour leschoses essentielles. Le cabinet d'anatomie n'était point d'une architecture convenable à un tel monument; le local en était beaucoup trop resrerré pour qu'il fût possible d'y mettre en ordre les nombreux objets dont M. Cuvier l'avait enrichi. On en a triplé l'étendue en y ajoutant les bâtimens voisins dont la distribution a été adaptée au but qu'on se proposait. Les grands squelettes ont été placés dans une vaste salle au rez-de-chaussée, et les galeries du premier étage, pour lesquelles on a construit un très-bel escalier, ont été divisées en une suite de pièces où tous les objets sont. classés méthodiquement et de la manière la plus favorable à l'étude. Cet édifice ayant été terminé en 18 I 7 , on s'est occupé en i 8 I 8 de la construction d'une ménagerie des animaux féroces, qui avait été projetée depuis long-temps, et qui a été enfin décidée par M. Laîné. La première pierre en a été posée le 25 mars I8I8, et les travaux ont été continués avec tant d'activité qu'elle a été ter- 
minée en dix-huit mois, et qu'on a pu au printemps de I82 I y placer les animaux et les exposer à la vue du public ; cet édifice, d'une architecture simple et régulière, forme une très-belle décoration dans la partie de la ménagerie voisine du quai, et correspond à la serre tempérée qui est située à l'autre extrémité. Dans le même temps, on a acquis quelques terrains pour les parcs des animaux paisibles, et l'on doit espérer qu'on y réunira bientôt tous ceux qui vont jusqu'à la rue de Seine. On va s'occuper de la construction denouvelles serres, devenues nécessaires pour la conservation et la multiplication des végétaux étrangers. Celles qui existent ont besoin de réparations continuelles, elles ne. sont nullement dignes de la grandeur et de la beauté de toutes les autres parties du Muséum (I).

M. de Buffon avait obtenu du Roi que des naturalistes seraient envoyés dans les pays étrangers, et les royages de Commerson, de Sonnerat, de Dombey, de Michaux avaient procuré au Jardin et au cabinet des collections considérables. Depuis la nouvelle organisation, les deux expéditions commandées par le capitaine Baudin ont tout à coup

(1) Un très-bel envoi de végétaux de l'Inde et de Cayenne, que nous arons reçu en août 1821,2 mis dans la nécessité de construire une petite serre au-devant de celles qui sont en face de l'école; mais il est évident que cette construction, faite a la hate, ne peut ctre considérée que comme provisoire. 
doublécesrichesses, et mis dans lanécessité d'augmenter le local où elles devaient être placées. Lorsque la paix a été rendue à la France, le Roi a accordé au Muséum les mêmes avantages, il ne s'est. pas borné à fournir des fonds pour les constructions intérieures, il a voulu que des voyageurs allassent parcourir les pays les moins connus pour y prendre des renseignemens sur toutes les partics de l'histoire naturelle. Déjà nous avons reçu des envois très-considérables qui nous ont été faits de Calculta et de Sumatra par MM. Diard et Duvaucel, de Pondichéry et de Chandernagor par M. Leschenault, du Brésil par M. Auguste Saint-Hilaire, de l'Amérique septentrionale par M. Milbert; et M. de Lalande, qui était allé au Cap et qui a pénétré fort avant dans l'intérieur des terres, nous a rapporté la collection de zoologie la plus nombreuse qui fût arrivée depuis celle de Péron.

D'autres royageurs qui n'avaient point une mission spéciale se sont empressés de donner des preuves de leur zèle pour les sciences. M. DussumierFonbrune, négociant de Bordeaux, nous a envoyé beaucoup de choses des Philippines; M. Stéven, savant naturaliste au service de Russie, qui a passé douze ans dans la Tauride et le Caucase, a donné au cabinet de botanique un grand nombre de plantes de cette contrée, et M. Dumont d'Urville, lieutenant de raisseau, celles qu'il a recueilic:s 
dans les îles de l'Archipel et sur les bords du Pont-Euxin; M. Freycinet, arrivé récemment de son voyage aux terres australes, nous a remis une collection en tout genre faite par les naturalistes de l'expédition ( I). M. Philibert, capitaine de vaisseau, ayant été chargé par le gouvernement de parcourir les mers d'Asie, et d'aller ả la Guiane française, a pris sur son bord M. Perrottet, jardinier du Muséum, et il a donné à ce jeune homme tant de facilités pour faire des recherches et pour en conserver le produit, qu'à son retour, au mois d'août dernier, il nous a remis i 58 espèces d'arbres et arbustes, ayant depuis six pouces jusqu'à cinq pieds d'élévation, et dont la plupart n'existent dans aucun jardin de l'Europe (2). A cette collection végétale, la plus précieuse qui nous soit jamais parvenue, étaient joints quelques oiseaux rares, et le gymnote, ce poisson si célèbre par la faculté qu'il a de donner à volonté de violentes commotions électriques. Enfin M. le baron Milius, ex-commandant pour le Roi à l'île de Bourbon, vient de nous rapporter de celte île quelques animaux vivans, et divers objets d'histoire naturelle.

Jusqu'à présent ces résultats, dus à la généro-

(2) M. Gaudichaud pour la botanique, M. Quoy et M. Gaimard pour la zoologie et la minéralogie.

(2) Les végétaux de Cayenne avaient été remis à M. Perrottet, par M. Poiteau, directeur du jardin de naturalisation dans cette colonie. 
sité du gouvernement et au zèle de quelques particuliers, avaient eu lieu à des époques indéterminées, et lorsque des circonstances favorables mettaient dans le cas de les solliciter; une nouvelle mesure vient de nous assurer qu'ils se reproduiront régulièrement toutes les années, et qu'ils seront spécialement appliqués aux besoins de l'établissement.

D'après un plan soumis au Roi par M. le duc Deeazes, un fonds annuel de 20,000 fr. a été destiné pour attacher au Muséum des élèves voyageurs. Ces élèves sont nommés sur la présentation des professeurs qui les ont d'abord examinés pour s'assurer de leur capacité et de leur instruction. Pendant la première année, ils doivent, sous la direction des mêmes professeurs, se préparer à bien remplir la mission qui leur sera confiée. Ils seront ensuite envoyés successivement dans les pays où l'on croit qu'il y a des objets à recueillir ou des découvertes à faire, On choisira parmi eux pour les diverses contrées, celui dont les connaissances ont le plus d'analogie avec le but qu'on se propose. Ils seront chargés d'entretenir une correspondance active avec le Muséum, pour faire parvenir et pour naturaliser au delà des mers les productions de notre agriculture.

Malheureusement le premier usage que nous ayons fait d'un moyen qui nous promet de si 
grands résultats, a été pour nous une source de regrets. De trois voyageurs partis en 1820 , deux ont été victimes de leur zèle en arrivant à leur destination. M. Godefroy, que des connaissances trèsvariées mettaientà même de nous rendre de grands services, a été tué dans une émeute des naturels du pays, peu de jours après son débarquement à Manille. M. Havet, jeune homme également distingué par son esprit, son instruction et / son caractère, est mort à Madagascar à la suite des fatigues auxquelles il s'est livré pour remplir sa mission. Avant son départ il avait étudié la langue madécasse ; il était recommandé à l'un des souverains de l'île, qui fait élever son fils à Paris, et nous espérions qu'il nous ferait connaitre les productions de cette contrée, dont l'intérieur n'a jamais été visité par des naturalistes (I). De telles pertes sont bien difficiles à réparer.

Cependant ces accidens funestes n'affaiblissent point l'ardeur de ceux qui ont formé le projet d'entrer dans la même carrière. Tous les jours de nouveaux élèves sollicitent la facilité d'aller parcourir les pays les moins civilisés. La vue des collections qui arrivent au Muséum excite leur enthousiasme; animés par l'espoir d'attacher leur nom à quelque découverte, ils ne comptent pour

(1) Le troisième de ces voyageurs, M. Plée, est allé aux Antilles, d'où il nous a fait plusieurs enrois. 
rien les plus grands sacrifices, et si nous leur peignons les privations et les dangers auxquels ils s'exposent, si nous lewr rappelons le sort de plusieurs de ceux qui les ont précédés, ils nous répondent comme Euryale à Nisus :

Mene igitur socium summis adjungere rebus,

Nise, fugis ? . . ...........

Est hic, est animus lucis contemptor, et istum

Qui vita bene credat emi, quo tendis honorem.

Nous venons de tracer le tableau des accroissemens successifs du Muséum, il nous reste à dire quelques mots de l'enseignement et des professeurs qui en ont été chargés depuis la nouvelle organisation.

La chaire de minéralogie fut d'abord remplie par M. Daubenton, qui, pendant vingt ans, avait professé cette science au collége de France, et qui se trouvait heureux de continuer sesleçons dans un lieu où il pouvait montrer à ses élèves les objets qu'il avait décrits. Malgré son grand âge, il fit toutes les années son cours avec une exactitude rigoureuse, ce qui ne l'empêcha point de rester fidèle à ses ancienneshabitudes, et de donner tous les jours des instructions particulières à ceux qui venaient le consulter. D'après ce que nous avons dit précédemment, on a vu que c'est à sa coopération avec Buffon que le Muséum doit son exis- 
tence, et l'histoire naturelle les progrès rapides qu'elle a faits dans le dernier siècle. C'était lui qui avait réuni et mis en ordre presque toutes les richesses de l'ancien cabinet.Lorsqu'il ne fut plus chargé que de la collection de minéralogie, il en prit un soin particulier; il passait les matinées au cabinet soit pour arranger les échantillons, soit pour répondre aux questions qui lui étaient adressées, soit pour recueillir des observations et pour s'informer des découvertes faites par ses anciens élèves, On écoutait avec respect ce patriarche de l'histoire naturelle, qui, à l'âge de quatre-vingt-quatre ans, les mains et les pieds déformés par la goutte, avait conservé toute la netteté de ses idées, toute l'activité de son esprit, et cette absence de préjugés qui lui faisait toujours accueillir la vérité.

Les professeurs qui, lors de leur première, assemblée, l'avaient nommé dirécteuir de l'établissement, ayant voulu deux ans après qu'il conservât ce titre et qu'il en remplît les fonctions, il refusa cet honneur parce que c'eût été une infraction au règlement, et que cet exemple aurait pu être dangereux; mais tout se faisait par ses conscils. Étranger aux dissensions politiques, il ne s'était jamais distrait de ses utiles travaux. La modération de son caractère avait assuré sa tranquillité ; et sa réputation, à laquelle il ne pensait pas, s'é- 
tait accrue d'année en année. Le gouvernement venait de lui donner la preuve de considération la plus flatteuse en le nommant membre du premier corps de l'état, et la prospérité qui se préparait pour le Muséum devait bientôt réaliser ses projets et combler ses voux, lorsqu'il fut frappé d'une attaque d'apoplexie dont il mourutle 3 I décembre r 799. Il fut inhumé dans le lieu même où il avait passé sa vie et où tout rappelle les services. qu'il a rendus.

Le 6 janvier suivant, les professeurs du Muséum, usant pour la première fois du droit qu'ils avaient de choisir leurs collègues, nommèrent M. de Dolomieu, qui s'était depris long - temps acquis une grande réputation comme minéralogiste, et surtout comme fondateur de la géologie en France. Ce savant, que la passion des connaissances avait déterminé à se joindre à l'expédition d'Égypte, avait été fait prisonnier à son retour, et on l'avait plongé dans un cachot à Messine, parce qu'on avait faussement supposé qu'il n'était point étranger à l'invasion de Malte. Les puissances de l'Europe n'ayant pu réussir à briser ses fers, ni même à adoucir sa captivité, les professeurs ignoraient s'il serait bientôt libre de se rendre auprès d'eux ; mais ils aimèrent mieux s'exposer à ce que la chaire ne fût point remplie pendant quelque temps, que de laisser échapper l'occasion 
de rendre une justice éclatante à un homme que son dévouement pour les sciences et l'élévation de son caractère n'avaient pu mettre à l'abri des calomnies les plus absurdes et des persécutions les plus odieuses. Cet hommage rendu au mérite parutégalement honorable pour ceux qui en étaient les auteurs et pour celui qui en était l'objet. M. de Dolomieu n'en fut informé que lorsque la liberté lui fut rendue le 5 mars $180 \mathrm{I}$, par un article $\mathrm{du}$ traité entre la France et Naples. Il se hâta de se rendre à Paris, et lorsqu'il parut dans l'amphithéâtre pour faire sa première leçon, il fut accueilli avec un enthousiasme qui montrait à la fois l'idée qu'on avait de ses connaissances, et l'intérêt qu'avaient inspiré ses malheurs, Après avoir fini son cours, il voulut profiter du reste de la belle saison pour parcourir les Alpes de la Suisse et du Dauphiné, d'où il espérait rapporter des minéraux pour le cabinet. Mais sa santé affaiblie par les maux qu'il avait éprouvés, ne put résister aux fatigues de ce voyage. A son retour, ils'arrêta à Neufchâtel en Charollais, chez M. le comte de Drée son beau-frère; et il fut atteint d'une maladie dont il mourut le 26 novembre 1801 .

Depuis plusieurs années les travaux ingénieux de Romé de l'Isle et de Bergmann avaient appelé l'attention sur la forme régulière et constante des cristaux; mais les observations qu'on avait faites 
ne présentaient que des phénomènes isolés. M. Haiiy ayant deviné la cause de cette structure, appela la géométrie à son secours pour en déterminer les lois, et bientôtil parvint à des résultats généraux qui devaient changer les bases de la science. Après avoir démontré cette grande découverte, il en fit l'application à toutes les espèces connues, dans le Traité de minéralogie qu'il publia en I 800 . Cet ouvrage, en établissant la distinction et la classification des espèces minérales, non plus uniquement sur des apparences extérieures, mais sur dès caractères essentiels qui dépendent de la nature même des molécules primitives, donna à la science 'une impulsion semblable à celle que trente ans auparavant les découvertes de Lavoisier avaient donnée à la chimie ; il l'assujettit de même à une marche uniforme et à une nomenclature régulière, avec cette différence que de nouvelles expériences peuvent modifier la théorie et la nomenclature de Lavoisier, tandis que les lois de la cristallisation sont invariables, rigoureusement déterminées par le calcul, et d'une application toujours sûre par la mesure des angles. M. Haiiy fut donc appeléà remplir une chaire pour laquelle il ne pouvait plus avoir de concurrens. Il fut nommé le 18 décembre ı 80 . Dès lors l'enseignement prit une nouvelle direction; il fut fait d'après la méthode cristallographique de ce 
savant, qui est le fondateur et le chef de l'école. française. On craignait d'abord que cette méthode ne convînt point à ceux qui ne s'étaient pas préparés à l'entendre; mais M. Haiiu a su s'aider de tant de secours, que toutes les difficultés ont été aplanies. Il a fait d'abord construire des modèles de cristaux pour rendre sensibles à l'œil les lois de décroissement et les modifications que peut subir la forme primitive: en présentant ensuite aux élèves les minéraux dans leur état de pureté, il leur a appris à distinguer les variations produites par le mélange de diverses substances. Il a fait sous leurs yeux les expériences les plus curieuses pour leur montrer les phénomènes qui résultent de l'action de l'électricité, du magnétisme et de la lumière; phénomènes nouvellement observés, et qui, différant selon les espèces, offrent pour les distinguer des caractères précis et dépendans de leur nature intime. L'ordre qu'il a établi dans la collection, donne le moyen de comparer les divers minéraux, d'observer toutes les nuances de forme, tous les passages d'une variété à l'autre. Au-dessus et au-dessous des tablettes où sont rangés les échantillons d'étude qui présentent sensiblement le caractère essentiel, sont placés d'autres morceaux d'un volume plus considérable pour qu'on puisse les reconnaître à l'aspect.

M. Haiiy possède lui-même une suite presque 
complète de cristaux qui lui servent d'exemples dans ses leçons, sans qu'il soit obligé de déplacer ceux qui sont dans les armoires du cabinet. Les instructions qu'il donne à ses élèves ne se bornent point à ses leçons publiques; ceux qui se livrent à l'étude de la minéralogie sont toujours sûrs d'être accueillis chez lui lorsqu'ils ont quelques éclaircissemens à lui demander.

L'influence de cet enseignement s'est répandue dans les pays étrangers. Les Allemands n'ont point renoncé à leur classification, mais ils associent les nouveaux caractères à ceux qu'ils avaient anciennement adoptés, et plusieurs ouvrages ont été publiés pour mettre en accord les principes de Wer" ner et ceux de M. Hauiy, l'école allemande et l'école française.

Lors de la nouvelle organisation de l'établissement, M. Desfontaines n'eut rien à changer à la méthode qu'il avait introduite en 1786 ; son cours se compose toujours de deux parties, dont une est consacrée à la physique végétale, l'autre à l'exposition des familles, des genres et des espèces les plus intéressantes. Ce cours a lieu trois fois par semaine pendant les mois de mai, juin, juillet et août; il est généralement suivi par cinq ou six cents élèves. Les instructions que donne le professeur ne se bornent point à la leçon faite dans l'amphithéâtre, elles résultent encore du soin qu'il 
I 40 HISTOIRE DU MUSEUM.

prend chaque jour de s'assurer qu'il ne s'est point glissé d'erreurs dans l'école, et d'y faire placer à leur étiquette, toutes les fois que le temps le permet ou du moins les jours de lecon, les plantes qu'on est obligé de conserver habituellement dans les serres.

De toutes les parties de $1^{\prime}$ histoire naturelle, la botanique est celle qui convient le mieux aux femmes. Les travaux qu'elle exige ne sauraient blesser leur délicatesse; clle leur offre un amusement dans la retraite; elle répand de l'intérèt sur leurs promenades; elle les attache à la culture des jardins; elle les met à même de développer chez leurs enfans le talent de l'observation en fixant leur attention sur des objets agréables; elle leur donne enfin le moyen de satisfaire leur goût pour la bienfaisance en faisant connaître aux habitans des campagnes les plantes qui croissent autour d'eux, et qui peuvent être utiles. Les Lettres de Rousseau leur avaient d'abord donné le goût de la botanique, et ce goût est devenu plus vif par la facilité qu'elles ont eu de s'instruire. On en voit un assez grand nombre se rendreau Jardin du Roi dès sept heures du matin pour assister au cours, et.1'on a cru devoir leur réserver dans l'amphithéâtre une enceinte séparée des gradins où se placent les hommes.

M. de Jussieu a toujours continué depuis $177^{\circ}$ 
à faire, pendant la belle saison, des herborisations à la campagne; elles sont suivies par un grand nombre d'élèves.

Le cours de culture est fait par M. Thouin, avec tous les développemens possibles, parce que les diverses écoles établies dans le Jardin, les pratiques suivies dans les serres etles couches, et les modèles des différens outils de culture mettent le professeur à même de joindre chaque jour l'exemple au précepte.

M. Thouin est encore chargé de la correspondance avec tous les jardins publics de France et des pays étrangers; et c'est sous sa direction que se fait toutes les années la distribution des plantes qu'on désire propager, et celle des graines recueillies dans les diverses parties du jardin, ou prises des envois qu'on a reçus des royageurs.

M. de Fourcroy, nommé professeur en 1784 , avait, dès ses premières leçons, inspiré l'enthousiasme de la chimie; les nouvelles découvertes qu'il avait d'abord annoncées en ayant produit d'autres, il s'empressa de les faire connaître et de les lier à la théorie générale. Chaque année ses leçons et ses ouvrayes présentaient des développemens plus étendus et des applications plus utiles. Après la suppression des universités, le Muséum étant resté le scul établissement consacré au progrès dessciences, M. de Fourcroy redoubla de zèle 
pour soutenir le rnouvement qu'il avait imprimé ; son activité semblait augmenter à mesure qu'elle s'exerçait sur un plus grand nombre d'objets. Quoique sa célébrité l'eût fait appeler à diverses fonctions, il trouva le moyen de suffire à tout, et il continua de faire ses cours avec la même exactitude. Mais lorsqu'il fut nommé au conseil d'état, et chargé de la direction de l'instruction publique, il se trouva dans l'impossibilité de disposer de son temps, et il fut obligé de se faire suppléer pour une partie de ses leçons. Il choisit pour cela son élève et son parent M. Laugier, qui depuis quelques années était attaché au Muséum avec le titre d'aide-naturaliste chargé des analyses. Celui-ci fit le cours pendant quelques années avec beaucoup de succès, età la mort de M. de Fourcroy, que nous eûmes le malheur de perdre en 1809 , à l'âge de cinquante-cinq ans, il fut nommé professeur titulaire. Il rappelle la méthode de son maître en exposant avec clarté, non-seulement les connaissances qu'il avait reçues de lui, mais celles qui résultent des rapides progrès que la chimie a faits depuis vingt ans.

Laplace d'aide-naturaliste qu'occupait M. Laugier, fut donnée à M. Chevreul, qui a inséré plusieurs mémoires dans nos Annales, et qui est l'a uteux de la partie chimique du Dictionnaire des sciences naturelles. 
L'enseignement de la chimie au Jardin du Roi, était autrefois confié à deux savans dont un, sous le nom de professeur, exposait la théorie, et l'autre, sous le nom de démonstrateur, était chargé de faire les expériences : il en résultait souvent de la discordance entre les leçons, et chacun des deux cours était incomplet; car les expériences doivent. suivre immédiatement les principes dont elles prouvent la vérité : aussi la place de démonstratrateur n'avait été utile que parce qu'elle avait été remplie par d'habiles chimistes, qui s'écartaient du but de l'institution en expliquant les faits d'après leurs propresidées. Lorsqu'à cette place on substitua une chaire pour l'enseignement des arts chimiques, elle appartint de droil à Mi. Brongniart qui avait succédé à Rouelle le jeune en 1779. M. Brongniart remplit d'autant mieux ses nouvelles fonctions, que dans ses leçons au Jardin et dans celles qu'il faisait comme professeur à l'Ecole de Pharmacie et au Lycée des Arts, il s'était toujours plus attaché à montrer les procédés et les applications utiles de la science qu'à exciter la curiosité par des phénomènes surprenans. A sa mort, arrivée au mois de février 1804 , il fut remplacé par M. Vauquelin, qui, ayant fait une étude spéciale des arts chimiques, put donner à cette partie importante de lá science un développement qu'elle n'avait jamais eu. On convient géné- 
ralement que par la lumière qu'il a répandue sur la chimie analytique, par ses travaux sur la docimasie, par la découverte du chrôme et de plusieurs autres substances, par la méthode raisonnée à laquelle il a soumis les procédés, M. Vauquelin a puissamment contribué aux progrès qu'ont faits nos manufactures depuis, qu'il a commencé de professer au Muséum.

Dès le commencement du dernier siècle, la botanique fut cultivée avec succès: On avait réuni beaucoup de plantes au Jardin du Roi, on avait fait des herbiers très-nombreux, et Tournefort put examiner et comparer tous les végétaux connus, établir des genres et les distribuer d'après une méthode qui conserve la plupart des rapports naturels. La zoologie n'avança point avec la même rapidité, non qu'elle fût négligée, mais parce qu'on n'avait pas les mêmes ressources. On décrivit isolément plusieurs animaux, on donna des détails curieux sur les insectes, et Linnæus dans son Systema, présenta dans un ordre régulier, et caractérisa dans un langage aussi précis que pittoresque, toutes les espèces qu'on avait observées jusqu'à lui. Cependant, la plupart des animaux des deux continens n'étaient connus que d'une manière vague, parce qu'on n'avait pu les rapprocher pour les comparè les uns auxautres, et pour déterminer les changemens que l'àge, la saison 
TROISIEME ÉPOQUE.

145

et d'autres circonstances produisent dans les mêmesindividus. Ce furent les collections du Jardin du Roi et les ouvrages dont elles facilitèrent l'exécution qui donnèrent à la zoologie une exactitude qu'elle n'avait jamais eue. L'histoire des quadrupèdes par MM. de Buffon et Daubenton, celle des oiseaux par MM. de Buffon et de Montbelliard, celle des cétacés et des poissons par M. de Lacépède firent connaître à fond les espèces que Linnæus avait indiquées, et un grand nombre d'autres dont on ignorait l'existence. Les mêmes collections offrirentà M. de Lamarck des matériaux pour l'histoire des animaux sans vertèbres, et à $\mathbf{M}$. Latreille le moyen de perfectionner son grand travail sur les insectes. Bientôt après, M. Cuvier fit pourla zoologie ce que M. de Jussieu avait fait pour la botanique, en établissant sur les rapports naturels, indiqués par des caractères invariables, une classification qui fut généralement adoptée; et l'on peut dire que la réunion dans un même licu des trois chaires de zoologie à une chaire d'anatomie comparée, établit en France une nouvelle école, où l'on apprit à distinguer les caractères du premier ordre qui constituent les familles et les genres, à comparer et à déterminer les espèces, et que c'est vraiment aux cours faits au Muséum, que la zoologie doit les immenses progrès qu'elle a faits depuis vingt-cinq ans. 
Les trois chaires sont occupées aujourd'hui par les mêmes professeurs qui furent appelés à les remplir lors de la création, et le nombre des élèves est plus considérable chaque année, soit parce que le goût de la zoologie est devenu plus général, soit parce que l'accroissement des collections et l'augmentation de la ménagerie offrent de nouveaux moyens de rendre l'instruction plus positive et plus étendue.

M. Geoffroy de Saint-Hilaire a été pendant quatre années l'un des savans employés dans l'expédition d'Égypte; son séjour dans cette contrée a été très-utile aux progrès de la science, non-seulement à cause des objets qu'il en a rapportés, mais parce que les observations qu'il a recueillies, et les méditations auxquelles il s'est livré l'ont conduit à faire des modifications importantes à la méthode qu'il avait d'a bord adoptée pour ses leçons.

Après avoir décrit les animaux en s'attachant aux caractères apparens, il a cru devoir faire envisager à ses élèves la zoologie sous un point de vue plus général, en professant une doctrine qui s'applique à toutes les parties et qui les lie entre elles. Cette doctrine repose sur quatre considérations, $\mathbf{I}^{\circ}$ la théorie des analogues; $2^{\circ}$ le principe des connexions; $3^{\circ}$ le balancement dans le volume des organes; $4^{\circ}$ les affinités électives des élémens 
TROISTEME ÉPOQUE.

organiques; c'est-à-dire sur les quatre vues principales de sa philosophie anatomique.

D’après cette idée qu'il développe maintenant dans ses cours, il ne se borne plus à indiquer les formes extérieures, il en montre la cause dans des modifications qui ne détruisent point les lois essenticlles et primitives, et il cherche ainsi à découvrir le plan que la nature a suivi dans l'organisation des différentes classes d'animaux.

M. Geoffroy avait été, depuis dix - huit mois, chargé seul d'enseigner l'histoire entière des animaux vertébrés, lorsque la loi du I I décembre 1794 créa, d'après la demande de tous les professeurs, une chaire particulière pour l'histoire des quadrupèdes ovipares, des reptiles et des poissons. M. de Lacépède, qui avait quitté le Jardin depuis deux ans, ayant été appelé à la remplir, au mois de janvier $179^{5}$, il ne se contenta point de faire régulièrement ses cours, il reprit avec ardeur ses anciens travaux pour la classification, Ia nomenclature, et l'arrangement des collections. Il s'occupa d'abord de celle dont il était spécialement chargé ; mais peu de temps après, à l'époque où la galerie supérieure du cabinet venait d'être terminée, son collègue, qui partait pour l'Égypte, l'ayant prié de le remplacer, il disposa dans le plus bel ordre la collection d'oiseaux formée de ceux de l'ancien cabinet, de ceux qu'on avait re - 
cus de Hollande, de ceux qui avaient été rapportés des Antilles par le capitaine Baudin, et d'Afrique par M. Levaillant; et ce fut par ses soins que cette collection, la plus magnifique qu'on eût réunie jusqu'alors, fut exposée à la vue du public, et devint classique pour l'étude de l'ornithologie.

La réputation que M. de Lacépède s'était acquise par ses ouvrages, l'empressement qu'on avait d'entendre l'ami et le continuateur de Buffon, attirèrent à ses leçons plusieurs jeunes naturalistes, qu'il détermina à s'attacher à une partie de l'histoire naturelle, qui jusqu'alors avait été négligée en France. Depuis dix ans il consacrait tous ses momens à faciliter l'étude de la science à laquelle il avait fait faire tant de progrès, lorsqu'il fut nommé à une place importante dont les fonctions ne lui laissaient plus de loisir. Il fut alors obligé de se faire suppléer, et de se borner à prononcer de temps en temps quelques discours sur les principes généraux et sur les résultats de l'histoire naturelle; mais en choisissant pour son suppléant M. Duméril, l'auteur de la Zoologie analytique, et le coopérateur de M. Cuvier dans les premiers volumes de son Anatomie comparée, il mit ses élèves à même de recevoir l'instruction la plus solide.

M. le chevalier de Lamarck, si recommandable par ses travaux sur les animaux sans vertèbres, a, pendant vingt-cinq ans, professé l'histoire des 
mollusques, des crustacés, des insectes, des vers et des zoophytes, qui n'avait jamais été dans son ensemble le sujet d'un cours particulier. Il a classé dans le cabinet les coquilles et les polypiers, d'après une méthode plus savante et plus exacte que toutes celles qu'on avait adoptées avant lui; il a caractérisé tous les genres, et déterminé un grand nombre d'espèces vivantes et d'espèces fossiles; et depuis que sa vue affaiblie ne lui permet plus de montrer les objets qu'il a si bien étudiés, il est suppléé dans ses leçons par M. Latreille, que ses nombreux écrits, et principalement son grand ouvrage sur la classificalion et les caractères génériques des crustacés et des insectes, ont placé au premier rang parmi les entomologistes de l'Europe.

Les trois cours de zoologie dont nous venons de parler, ont lieu pendant la belle saison et durant trois ou quatremois. Ils se font dans les salles du cabinet où les élèves ont sous les yeux les objets dont parle le professeur, et peuvent les examiner encore après la leçon.

La chaire d'anatomie humaine, établie au Jardin du Roi peu de temps après sa fondation, a toujours été remplie par des savans du premier ordre, et pendant bien desannées elle fut considérée comme celle où l'enseignement était le plus complet. Dans les temps modernes, et lorsque les cours d'anato- 
150 HISTOIRE DU MUSÉUM.

mie se sont multipliés, elle n'a point dégénéré de son ancienne réputation. Elle est depuis 1778 occupée par M. Portal, aujourd'hui premier médecin de sa majesté, et président de l'académie de médecine. Ce savant n'a jamais interrompu ses cours, et il les fait toujours avec le même zèle.

M. Mertrud, qui avait pendant plusieurs années travaillé à la dissection des animaux avec M. Daubenton, n'avait pu cependant considérer l'anatomie de ce point de vue élevé qui lui permet d'embrasser les rapports de tous les animaux, depuis le polype jusqu'à l'éléphant, et de comparer dans chaque classe les organes essentiels. M. Cuvier, nommé suppléant de M. Mertrud, le 15 novembre $179^{5}$, et professeur titulaire après la mort de celui-ci au $\mathrm{I}^{\mathrm{er}}$ novembre 1802 , enseigna cette science dans son ensemble et dans ses détails; il forma le cabinet d'anatomie, dans lequel il plaça les squelettes entiers de tous les animaux qu'il put se procurer, la collection des os de même nature pris de chaque animal, et les différens systèmes d'organes intérieurs préparés et conservés dans l'esprit-de-vin. Il profita pour cela des ressources que lui offrait l'établissement de la ménagerie et des envois que les voyageurs et les naturalistes étrangers faisaient au Muséum. On sait que depuis cette époque l'anatomie comparée est devenue la principale base de la zoologie. 
L'établissement d'un cours de géologie, séparé du cours de minéralogie, est une innovation extrêmement avantageuse ( I). Sans doute, le géologue ne peut se passer des caractères précis que lui donne le minéralogiste, soit pour connaître parfaitement les genres et les espèces dans leur état de pureté, soit pour discerner dans les agrégats, les élémens dont ils sont formés, et les altérations que le mélange de diverses substances produit dans la forme primitive; mais l'histoire des grandes masses qui couvrent le globe, celle de la situation respective des roches et deleurs diverses formations, celle des feux souterrains et des produits volcaniques, celle des eaux thermales, celle des coquilles et des ossemens fossiles qu' on trouve à différentes profondeurs, composent une science particulière, fondée sur d'innombrables observations, et dont l'exactitude peut nous garantir des systèmes auxquels la théorie de la terre avait été abandonnée jusqu'ici.

M. Faujas de Saint-Fond fut le premier qui remplit la chaire qu'on venait de créer au Muséum, il fit d'une manière brillante le cours de géologie, et si cette science, malgré les faits dont il l'avait

(1) La géologie dont on s'occupe aujourd'hui beaucoup, était autrefois si peu étudiée, que le nom même n'en était connu que des savang, et n'entrait point dans la langue ordinaire. Le mot géologie ne se trouve point dans le Dictionnaire de l'académie quoiqu'on y trouve les mots zoologie et zoographie. 
152 HISTOIRE DU MUSÉUM.

enrichie par ses recherches, n'était point encore assez avancée pour qu'il pût la soumettre à des règles positives, il eut du moins l'honneur d'en inspirer le goût aux nombreux élèves qui suivaient ses leçons, et de préparer ainsi les progrès rapides qu'elle a faits depuis le commencement du siècle.

Pendant les dernières années de sa vie, l'affaiblissement de sa santé l'avait obligé à se retirer à la campagne, et quoiqu'il lui fût pénible d'être éloigné de ses collègues, quoiqu'il sentît la nécessité d'arranger dans les nouvelles salles du cabinet les nombreux objets qu'il avait rassemblés, il ne venait guère à Paris que pour faire son cours. II était âgé de soixante-dix-huit ans, lorsqu'il a terminé sa carrière à sa terre de Saint-Fond, près de Montelimar, le i 8 juillet I 8 ig.

Une ordonnance du Roi, rendue le I3 septembre suivant, d'après la présentation des professeurs du Muséum et de l'Académie dessciences, a nommé à la chaire de géologie M. Cordier, inspecteur divisionnaire des mines, l'élève et l'ami du célèbre Dolomieu, qu'il avait accompagné dans plusieurs de ses voyages, et dont il avait été le collègue dans l'expédition d'Égypte. Aussitôt après son entrée au Muséum, ce savant s'est occupé à mettre en ordre la collection de géologie; il en a formétrois séries, la première selon la nature des roches, la 
seconde selon leur gissement, la troisième selon les localités. Dans ses leçons, commencées en I 820, il a pris soin d'écarter les hypothèses, pour se borner à exposer les faits constatés par l'observation, et à les lier entre eux de manière à donner des notions exactes sur l'état actuel du globe. Il s'est surtout attaché à présenter le tableau des richesses que renferme le sol de la France, et à faire connaître les moyens de les exploiter et de les employer au progrès des arts et aux besoins de la société.

L'histoire naturelle ne peut se passer du secours du dessin, et les descriptions les plus exactes laissent toujours de l'incertitude lorsqu'elles ne sont pas accompagnées de bonnes figures; le langage exprime fort bien les caractères essentiels, mais il ne saurait offrir à l'esprit une image de la physionomie des êtres, de cet ensemble qui les fait reconnaître au premier coup d'oeil; et ce fut une heureuse idée d'attacher au Muséum un peintre qui enseignât cet art particulier de représenter les objets de la nature, non point uniquement pour produire un effet pittoresque, mais pour rendre avec exactitude les traits qui les distinguent. En répandant le goût du dessin, cette institution l'a dirigévers un but utile, et chacun peut remarquer combien les figures dont sont accompagnés aujourd'hui les livres d'histoire naturelle 
sont supérieures à celles dont ils étaient ornés dans le dernier siècle. Ce n'est pas qu'il n'y eût anciennement des artistes fort habiles, et les vélins du Muséum en offrent la preuve; mais ces artistes étaient en petit nombre, et les naturalistes trouvaient difficilement quelqu'un qui pût les seconder. Depuis que M. Vanspaendonck fait un cours public, il a formé de nombreux élèves qui, s'étant d'abord exercés à copier ses modèles, puis à dessiner sous sa direction les plantes du Jardin et les animaux de la ménagerie, ont contribué à rendre plus facile et plus sûre l'étude de la botanique et de la zoologic. Quoique les leçons du professeur aient pour but principal d'enseigner à saisir et à rendre les caractères qui distinguent les êtres naturels, ce qui tient à l'agrément n'est point négligé, et c'est peut être à cela qu'est dûle degré de perfection auquel s'estélevé en France l'art de peindre les fleurs, ct l'influence que cet art a exercé sur plusieurs de nos manufactures.

M. Vanspaendonck avait été attaché au Jardin dès I 774 , et l'on trouve dans les portefeuilles plusicurs vélins de lui. La célébrité dont il jouit attire à son cours beaucoup de jeunes demoiselles qui y sont conduites par leurs mères. Plusieurs d'entre elles ont acquis un talent distingué, et quelquesunes l'appliquant essentiellement à la botanique, nous ont donné d'excellentes figures de plantes, 
comme on peut le voir en parcourant celles qui sont gravées dans les Annales du Muséum.

Le cours d'iconographie a jusqu'à présent été fait à la bibliothèque; il a lieu pendant près de quatre mois, et trois fois par semaine. Les jours de leçon, la bibliothèque n'est ouverte qu'aux élèves qui se sont fait inscrire; dans les jours d'intervalle, plusieurs d'entre eux continuent leur travailaux heures de lecture, et M. Vanspaendonck. y vient souvent pour leur donner ses conseils.

Les professeurs que nous venons de nommer étant obligés de mettre l'enseignementà lá portée d'un grand nombre d'élèves, ils ne peuvent exposer dans leurs cours ni les observations de détail, ni les nouvelles découvertes dont la théorie et les rapports ne seraient bien saisis que par ceux qui ont déjà beaucoup d'instruction; mais les Mémoires qu'ils publient en commun leur offrent le moyen de répandre ces observations et ces découvertes chez tous ceux qui se livrent aux sciences. Ainsi M. Hauiy a profité de cet ouvrage, soit pour fixer les caractères de divers minéraux récemment arrivés au cabinet, soit pour exposer la simplicité des lois auxquelles est soumise la structure des cristaux, et les avantages des formules analytiques. MM. Fourcroy, Vauquelin et Laugier y ont inséré les résultats des travaux les plus importans faits dans le laboratoire de chimie. M. Desfontaines y 
a décrit les nouveaux genres de plantes qu'il a vues fleurir au Jardin ou qu'il a trouvées dans nos herbiers; M. de Jussieu y a donné les caractères des principales familles naturelles des plantes, avec les additions et les rectifications que le progrès de lascience l'a mis à même de faire à ceux qu'ilavait admis dans son ouvrage; M. Thouin y a expliqué en détail les pratiques adoptées au Muséum pour les semis, les plantations, la greffe; MM. Geoffroy et de Lacépède y ont publié des genres nouveaux de quadrupèdes, de chauve-souris, de reptiles et de poissons; M. de Lamarck y a décrit les coquilles fossiles des environs de Paris; M. Curier y a fait connaître l'anatomie des mollusques et le squelette des animaux perdus dont ila rassemblé les ossemens fossiles; tous se sont empressés d'y donner l'extrait de la correspondance que leur place au Muséum les oblige d'entretenir soit avec les établissemens du mème genre, soit avec les voyageurs et avec les savans étrangers.

Si dans chaque partie on est conduit par degrés des premiers élémens aux connaissances les plus élevées, il n'existe pas non plus de lacune entre les diverses parties de l'enseignement; toutes se lient pour concourir à un même résultat, celui d'assurer la marche progressive des sciences naturelles, et de les faire servir aux besoins et au bonheur de la société. 
TROISIEM ÉPOQUE.

Deux mille élèves suivent chaque année les cours du Muséum; quelques-uns seulement deviennent des naturalistes distingués ; maisil n'en est pas un qui n'apprenne des choses utiles, et qui n'acquière le talent de l'observation. Bacon disait qu'en philosophie l'ignorance était préférable au demisavoir; et cela est vrai; car un esprit faux peut employer des notions superficielles d'histoire ou de philosophie pourattaquer les principes fondamentaux de la morale et de la politique; mais il n'en est pas de même de la connaissance de la nature : dans cette science illimitée tout est immédiatement utile, depuis les notions les plus simples jusqu'aux recherches les plus profondes, et depuis les plus petits détails jusqu'aux vues générales. L'étude des sciences naturelles convient également à toutes les époques de la vie, à tous les états de l'âme, à toutes les professions : elle s'associe à tous les autres genres d'études; elle a de l'intérêt dans toutes les circonstances, au milieu du luxe des villes comme dans la solitude de la campagne; elle amuse l'enfance, et procure à la vieillesse des jouissances paisibles; elle offre des secours à l'agriculture, à la médecine et aux arts, et contribue puissamment à la richesse des nations. Comme elle a pour but de constater les faits et de les coordonner, et non d'en chercher l'explication, elle n'est point hypothétique; et si 
l'observation est quelquefois incomplète, la nature est toujours là pour dissiper les doutes et rectifier les erreurs. Mais pour que cette étude ait les résultats qu'on en doit attendre, il faut qu'elle soit bien dirigée, il faut épargner à ceux qui commencent les recherches pénibles qu'ont faites ceux qui nous ont précédés, il faut qu'il y ait un dépôt de toutes les connaissances acquises, où chacun aille puiser, pour ensuite l'enrichir à son tour.

Ce dépôt existe chez nous: formé d'abord par nos rois, illustré dans la suite par des hommes de génie, et dirigé par des administrateurs éclairés, il a été organisé de manière à ce que l'ordre n'y fût jamais troublé; on l'a vu résister à toutes les secousses, échapper à toutes les dévastations, exciter l'admiration des étrangers; il a pris un nouvel accroissement depuis que la paix nous est rendue; son utilité garantit sa durée; et s'il nous fallait un motif de plus pour compter sur sa prospérité, nous le trouverions dans notre confiance en un monarque protecteur des sciences, et dont le progrès des lumières doit à jamais assurer la gloire, en faisant mieux sentir le prix des institutions qu'il a données à son peuple. 


\section{LIS TE}

\section{Des PRINCipaUX EMPLOYÉS DU MUSEUM, EN}

JANVIER I 822.

PROFESSEURS ET AIDE-NATURALISTES.

L'HistoIre que nous avons tracée de la fondation, des progrès et de l'état actuel du Muséum, fait connaître la nature des places qui y sont établies, et le nom de ceux qui ont été appelés à les remplir. Nous croyons cependant qu'on sera bien aise de trouver dans un article à part, la liste des professeurs, des aide-naturalistes, des gardes, des bibliothécaires, des peintres, des correspondans et des voyageurs qui y sont attachés en ce moment, avec la date de leur nomination et l'indication de leurs principaux ouvrages.

Nous suivrons pour cette liste l'ordre des chaires et des places établies par le décret qui a définitivement organisé le Muséum en $179^{5}$.

Nous n'indiquerons point le sujet des mémoires insérés dans les recueils des académies, dans les ouvrages périodiques et dans les dictionnaires, parce que ces détails nous entraîneraient trop loin. 


\section{MINÉRALOGIE.}

M. HA Ür (René-Just), né en I 743, à Saint-Just, département de l'Oise : chanoine honoraire de l'église métropolitaine de Paris (1); de l'académie des sciences en 1783; professeur à la Faculté des sciences de l'université; nommé au Muséum en décembre I80I.

Essai d'une théorie sur la structure des cristaux, I vol. in-8, Paris, 1788 .

Traité de minéralogie; Paris, 1800 , 4 vol. in- 8 , avec un atlas.

Traité des caractères physiques des pierres précieuses; Paris, 1819 , 1 vol. in-8.

Traité élémentaire de physique; 2 vol. in-8, Paris, trois éditions, la dernière de $\mathbf{1 8 2 1}$.

Un grand nombre de mémoires dans les Recueils de l'académie des sciences et de l'Institut, daris les Annales du Muséum, dans le Journal des mines, etc.

Aide-naturaliste, suppléant M. Haüy dans ses leçons.

M. dE la Fosse (Gabriel), né en I 796, à SaintQuentin, département de l'Aisne.

M. De la Fosse n'ayant encore publié aucun ourrage, il est

(1) Tous les professeurs dụ Muséum sont membres de la Légion d'honneur; tous sont associés ou correspondans des principales sociétés savantes de l'Europe et de l'Amérique : les bornes de cet ouvrage ne nous permettent pas d'énoncer pour chacun d'eux les titres qui prouvent la considération dont ils jouissent en France et dans les pays étrangers. 
PRINCIPAUX EMPLOYKS.

161

essentiel de faire connaître les motifs qui ont déterminé

M. Haüy à le choisir pour son suppléant, et l'assemblée du Muséum à autoriser ce choix. Nous croyons en conséquence devoir citer quelques lignss de ce que M. Haüy dit de lui dans son Traité de physique; Introduction, page $x \times x i r$.

" Nous avons eu tout lieu de nous féliciter d'aroir auprès de "nous M. de la Fosse...... Ce jeune savant nous a "puissamment secondé dans les expériences destinées à "vérifier les nouveaux faits que nous nous proposions de - publier..... Il a coopéré a la rédaction de plusieurs - articles.... On jugera par le Traité de cristallogra" phie qui va bientôt paraître, du succès arec lequel il a "cultivé cette branche importante de la minéralogie.

\section{CHIMIE GÉRERALE.}

M. Laugier (Ándré), né à Paris en $\mathbf{1 7 7 0}$, de l'académie de médecine.

Attaché au Muséum, avec le titre d'aide-naturaliste chargé des analyses, en juin 1803 ; choisi deux ans après pour suppléer M. de Fourcroy dans ses leçons; nommé professeur le 17 février 18 ro.

Plusieurs mémoires parmi ceux de l'Institut, de l'Académie des sciences, des Annales de chimie, et des Annales du Muséum.

Aide-naturaliste.

M. Dubois (Antoine-Charles), né à Paris en ז 776 .

Atiaché au laboratoire de chimie depuis 1796 . 
CHIMIE APPLIQUÉE AUX ARTS.

M. Vauguelin (Nicolas-Louis), né à Hibertot près Pont-l'Évêque, en I 763 .

Reçu membre de l'académie des sciences en I792; professeur à la faculté de médecine; directeur de l'école de pharmacie; nommé professeur au Muséum en 1804 .

De nombreux mémoires dans les recueils de l'Institut et de l'Académie des sciences, dans les Ánnales du Muséum, dans celles de Chimie, dans le Journal des mines, dans le Bulletin de la société philomatique.

Aide-naturaliste chargé des analyses.

M. Chevreul (Michel-Eugène), né à Angers en I 786 , nommé au Muséum en r8og.

Auteur de la partie chimique du Dictionnaire des sciences naturelles; d'un ouvrage sur les corps gras, actuellement sous presse; et de plusieurs mémoires insérés dana les Annales du Muséum.

\section{BOTANIQUE.}

M. Desfontaines (René-Louiche), né en 1752 , à Tremblay, département d'Ille-et-Vilaine.

Docteur de la faculté de médecine en 1782 ; de l'académie des sciences en 1783 ; professeur à la faculté des sciences de l'université; nommé professeur au Jardin du Roi en 1786.

Flora atlantica, 2 vol. in 4 , avec 260 planches, 1798 .

Tableau de l'école de botanique du Muséum, 1 vol. in-8, deux éditions 1804 et 1815 . 
Choix de plantes du Corollaire des instituts de Tournefort, 1 vol. in-4 avec figures; 1808 .

Histoire des arbres,et arbrisseaux qui peuvent être cultivés en pleine terre, 2 vol. in 8,1809 .

Un grand nombre de genres nouveaux et autres mémoires insérés dans les Annales du Muséum et dans̀ le recueil de l'Académie des sciences et de I Institut.

Nous devons citer comme ayant fait epoque celui sur l'organisation comparée des Monocotylédons et des Dicotylédons, imprimé en 1797 , dans le troisième volume des Mémoires de l'Institut.

Aide-naturaliste.

M. Delevze (Joseph-Philippe-François), né en I753, à Sistéron, département des Basses-Alpes.

Secrétaire de l'association formée par les professeurs pour la publication des Annales du Muséum. Nommé aide-naturaliste en février 1795.

Les Amours des plantes, de Darwin, 1 vol. in-12, 1799.

Saisons de Thompson, deux éditions, 1801 et 1806 , in -8 et in- 12.

Eudoxe, Entretiens sur l'étude des sciences, des lettres et de la philosophie, 2 vol. in-8, 1810 .

Histoire critique du magnétisme animal, 2 vol. in-8, première édition, 1815 ; deuxième édition, 1819 .

Défense du magnétisme, 1 vol. in-8, 1819 .

Plusieurs éloges historiques et quelques mémoires dans les Annales du Muséum, etc.

BOTANIQUE A LA CAMPAGNE.

M. DE Jussifu (Antoine-Laurent), né à Lyon en 1748 . 
I64 HISTUIRE DU MUSÉU.

Docteur de la faculté de médecine en 1772 ; de l'académie des sciences en 1773 , de la société royale de médecine en 1776 ; professeur à l'école de médecine depuis r8o4; suppléant de M. Lemonnier au Jardin du Roi, de 1770 à 1787 ; nommé démonstrateur à la mort de son oncle Bernard en I777; professeur titulaire depuis la nouvelle organisation.

Genera plantarum secundum ordines naturales disposita, 1 vol. in-8, Paris, 1789 .

De nombreux mémoires dans le recueil de l'Académie des sciences, et dans les Annales du Muséum; parmi ceux-ci se trouvent les caractères de plusieurs familles naturelles, et des monographies.

Un grand nombre d'articles dans le Dictionnaire des sciences naturelles, dont il est.l'un des principaux collaborateurs.

CULTURE.

M. Thourn (André), né au Jardiñdu Roi en I 747 .

De l'académie des sciences en r786; nommé jardinier en chef en I 768 ; professeur de culture au Muséum depuis la création de cette chaire lors de la nouvelle organisation.

Ses nombreux écrits, tous relatifs aux principes ou à la pratique de l'agriculture, sont insérés dans les Mémoires de l'académie des sciences, de l'institut, de la société d'agriculture; dans les Annales du Muséum, où il a donné la description du jurdin des semis, de l'école de culture, de celle des arbres fruitiers, etc.; dans le Dictionnaire 
d'histoire naturelle imprimé par Deterville; dans la nouvelle édition du Cours d'agriculture de Rozier.

Il a publié à part, Monographie des greffes, 1 vol. in-4, 1820 . Les tableaux manuscrits de ses leçons se trouvent a la bibliothèque du Muséum, oủ il est permis d'en prendre copie.

Aide-naturaliste.

M. Leclerc (Oscar), né à Paris en $\mathbf{1} 798$.

Attaché au Muséum en I8I8.

Il a coopéré à la deuxième édition de la Monographie des greffes de M. Thouin.

\section{Jardinier en chef.}

M. Thouin (Jean), frère du professeur, né au Jardin du Roi en 1756.

Attaché au Jardin depuis son enfance, et collaborateur de son frère; nommé jardinier en chef lors de la nouvelle organisation, et chargé en cette qualité de diriger les travaux, de faire les semis, de surveiller la récolte et la distribution des graines; membre de la Légion d'honneur.

ZOOLOGIE, MAMMIFÈRES ET OISEAUX.

M. Geofrroy-Saint-Hilaire (Étienne), né à Étampes en 1772 .

Membre de l'Institut en $\mathrm{r} 807$, ensuite de l'académie des sciences; professeur à la facultẹ́ des sciences de l'université; nommé professeur au Muséum lors de la nouvelle organisation.

Philosophie anatomique, 2 vol. in-8, 1818 et 1822 . 
Un grand nombre de mémoires de zoologie et d'anatomie comparée, dans les recueils de l'Institut ét de l'Académic des sciences, dans les Annales du Muséum, et dans la Description de l'Égypte.

Aide-vaturaliste pour la préparation des animaux.

\section{Delalande.}

Il a fait plusieurs voyages qui ont enrichi les collections du Muséum. Au retour du dernier, il a reçu la décoration de la Légion d'honneur. Son père, qui vient de mourir, avait monté la plupart des quadrupèdes du cabinet.

\section{ZOOLOGIE, REPTILES ET POISSONS.}

MI. le comte dE LACËPÈDE (Bernard-GermainEtienne), né à Agen en 1756 , pair de France, grand-croix de l'ordre royal de la Légion d'honneur.

De l'Institut lors de la création, ensuite de l'académie des sciences; nommé garde et sous-démonstrateur au Jardin du Roi en 1785 ; professeur lors de la création de la troisième chaire de zoologie en $179^{5}$.

Essai sur l'électricité, 2 vol. in-8, 1781.

Physique générale et particulière, 2 vol. in-12, 1782.

Poétique de la musique, 2 vol. in-12, 1787.

Histoịre des quadrupèdes ovipares et des serpens, faisant. suite a l'Histoire naturelle de Buffon, 2 vol. in-4, 1788 , 1789 , réimprimé in-12 et in-8.

Histoire naturelle des poissons, 5 vol. in- $4,1798,1803$, réimprimé in-12 et in-8. 
PRINCIPAUX EMPLOYÉS.

Histoire naturelle des cétacés, 1 vol. in-4, 1804 .

La Ménagerie du Muséum, avec M.M. Geoffroy et G. Cuvier, 1 vol. in-fol., réimprimé in 12.

Plusieurs mémoires dans les recueils de l'Académie des sciences et du Muséum, et dans le Dictionnaỉe des sciences naturelles.

M. de Lacépède est suppléé dans ses leçons depuis 1803 , par :

M. Dưméril (André-Marie-Constant), né à Amiens en 7774 , nommé à l'Institut en r8r3. Secrétaire de la section de médecine de la société royale de médecine.

Il a rédigé les deux premiers volumes des Leçons d'anatomie comparée de M. Cuvier.

Il a publié, Zoologie analytique, I vol. in-8, 1806 .

Traité élémentaire d'histoire naturelle, deux éditions; la première, 1 vol. in-8, 1804 ; la seconde, 2 vol. in-8, 1807. Plusieurs mémoires de zoologie, dans l'Eacyclopédie méthodique, dans le Bulletin de la société philomatique, etc.

Il s'est chargé des articles d'entomologie dans le Dictionnaire des sciences naturelles.

Aide-Naturaliste.

M. Valenciennes (Achille), néà Paris en 1794 . Nommé au Muséum en I812.

Il a inséré des mémoires parmi ceux du Muséum, et travaillé aveć M. de Humboldt au Recueil des observations de zoologie que ce savant publie par livraisons in-4, depuis 1811. 


\section{8}

HISTOIRE DU MUSËUM.

C'est lui qui, sous la direction de MM. les professeurs de zoologie et d'anatomie comparée, a été chargé, depuis. l'agrandissement du cabinet, de mettre en ordre et de nommer la collection des animaux vertébrés.

\section{ZOOLOGIE, ANIMAUX SANS VERTÈBRES.}

M. le chevalier DE LAMARCK (Jean-BaptistePierre-Antoine), né à Bazantin près Bapaume, en 1744 .

Membre de l'académie des sciences en 1779 ; attaché au Jardin du Roi avec le titre de botaniste du cabinet, en I 789 ; professeur de zoologie pour les animaux sans vertèbres lors de la création de la chaire.

Flore française, 3 vol. in- 8,1778 . Nourelle édition, augmentée par M. Decandolle, 5 vol. in-8, 1795.

Hydrogéologie, 1 vol. in-8, 1801 .

Recherches sur les causes des principaux faits physiques, 2 vol. in-8, 1794.

Système des animaux sans vertèbres, $x$ vol. in $-8,1801$.

Les premiers volumes du Dictionnaire de botanique, et les Illustrationes generum, faisant partie de l'Encyclopédie méthodique.

Philosophie zoologique, 2 vol. in-8, 1809 .

Système analytique des connaissances positives de l'homme, 1 vol. in-8, 1820 .

Histoire naturelle des animaux sans vertèbres, 7 vol. in-8, 1822 .

Un grand nombre de Mémoires parmi ceux de l'Institut, de l'Académie des sciences et du Muséum, dans le Jouraal d'histoire naturelle, dans celui de physique, etc. 
Aide-naturaliste, et professeur suppléant M. de Lamarck depuis $18 \mathrm{rg}$.

M. Latreilue(Pierre-André), néà Brive, département de la Corrèze, en I 762. Nommé à l'académie des sciences en 1814, entré au Muséum en I 797. C'est lui qui a classé et nommé la collection d'entomologie.

Histoire naturelle des salamandres de France, 1 vol. in-8, 1800.

Histoire naturelle et générale des fourmis, et Recueil de Mémoires, 1 vol. in-8, 1802 .

Genera crustaceorum et insectorum, 4 vol. in-8, 1809 et années suivantes.

Histoire naturelle des reptiles, faisant suite an Buffon de Castel, 4 vol. in-18.

Histoire naturelle des crustacés et des insectes, faisant suite au Buffon de Sonnini, 14 vol. in-8.

Le troisième volume, ou la partie entomologique de l'ouvrage de M. Cuvier, intitulé le Règne animal.

Un grand nombre de Mémoires parmi ceux de l'Académie des seiences et du Muséum, et les principaux articles d'entomologie du Dictionnaire d'histoire naturelle de Deterville.

Arde-naturaliste pour les animaux sans vertèbres, et chef des travaux du laboratoire de zoologie.

M. Dufresne (Louis), né à Champien, département de la Somme en $7_{7} 52$; nommé en juin 1793 . Auteur de l'article Taxidermie du Dictionnaire d'histoire na- 
turelle, ot d'un mémoire inséré dans les Annales du Muséum.

\section{ANATOMIE HUMAINE.}

M Portal (Antoine), né en $\mathbf{7} 7 \mathbf{4 2}$, à Gaillac, département du Tarn.

Docteur de la faculté de médecine de Montpellier en I 765 ; professeur d'anatomie au collége de France en 1768; membre de l'académie des sciences en r 769 ; président d'honneur de l'académie de médecine; premier médecin de Sa Majesté; chevalier de l'ordre de Saint-Michel ; professeur au Jardin du Roi depuis 1778 .

Les ouvrages de M. Portal sont en 'si grand nombre, que nous ne pouvons en donner ici le catalogue, nous nous bornerons à citer:

Histoire de l'anatomie et de la chirurgie, etc., 6 rol. in-8, $1 ; 70-17 \% 7$.

Observations sur la nature et le traitement de la rage, 1 vol. in-12, 1779 .

Observations sur les vapeurs méphytiques, sur les noyés, les asphyxiés, la rage, etc., 1 vol. in-8, 1791.

Instruction sur le traitement des asphyxiés, des noyés, etc.; 1 vol. in-12, réimprimé un grand nombre de fois par ordre du gouvernement.

Observations sur la nature etle traitement de la phthisie pulmonaire; première édition, 1 vol. in-8, 1792 ; deuxième "edition, 2 vol. in -8 , avec des notes traduites des autęurs allemands et italiens.

Observations sur la nature et le traitement du rachitisme, etc., 1 vol. in-8, 1796. 
Recueil de mémoires sur la nature et le traitement de plusieurs maladies, etc., 2 vol. in-8, 1800 .

Un grand nombre de mémoires dans les recueils de 1'Académie des sciences, de l'Institut, du Muséum, et dans divers journaux scientifiques.

\section{Aide-naturaliste.}

M. Martin (Jean-Paul), né en 1788, à Cahusac, département du Tarn; nommé au Muséum en i 809.

Il est spécialement chargé de faire les préparations nécessaires pour le cours; il occupe la même place au collége royal de France, sous le même professeur; il est membre du cercle médical.

\section{ANATOMIE COMPAREE.}

M. le baron Cuvier (Georges), né à Montbelliard en $176 \mathrm{~g}$.

Conseiller d'état, membre du conseil royal d'instruction publique ; professeur d'histoire naturelle au collége de France; nommé membre de l'Institut lors de la création; secrétaire perpétuel de la classe des sciences naturelles en I803; aujourd'hui l'un des deux secrétaires perpétuels de l'académie des sciences; membre de l'académie française en r818. Nornmé suppléant de M. Mertrud en $179^{5}$; professeur titulaire en 1802.

Tableau élémentaire du règne animal, vol. in-8, $179^{8}$. Leçons d'anatomie comparée, rédigées par MM. Duméril et Duvernoy, 5 rol. in-8. 
Rapport historique sur les progrès des sciences naturelles depuis 1789 , et sur leur état actuel, 1 rol. In-8, 1810 .

Recherches sur les ossemens fossiles, première édition en 4 vol. in-4, 1812 ; deuxième édition, 5 vol.ıin- $4,1822$.

Mémoires pour servir à l'histoire et à l'anatomie des mollusques, 1 vol. in- 4,1817 .

Le règne animal, 4 vol. in-8, 1817 .

Recueil des éloges historiques des membres de l'académie des sciences, 2 vol. in-8, $18 \mathrm{xg}$.

Rapport sur l'état de l'instruction publique en Hollande et en Italie, 2 vol, in 8.

Un grand nombre de Mémoires parmi ceux de l'Académie des sciences et du Muséum, et plusieurs articles dans le Dictionnaire des sciences naturelles et dans la Biographie universelle.

Aide-NATURALISTE.

M. Rousseau (Simon-Pierre), né à Belleville près Paris, en x 756. Attaché au Muséum en I $79^{5 .}$

Il a fait la plupart des squelettes et des préparations du cabinet d'anatomie comparée.

GARDE DES GALERIES D'ANATOMIE COMPARÉE.

M.Laurillard (Charles-Léopold), né à Montbelliard en 1784. Nommé en mars 1812.

Il a aidé M. Cuvier dans ses recherches, et a fait pour lưi un grand nombre de dessins de zoologie et d'anatomie, dont plusieurs sont gravés dans les ourrages que nous venons de citer. 
GÉOLOGIE.

M. Cordier (Pierre-Louis-Antoine), né à Abbeville en 1777 .

Inspecteur divisionnaire au corps royal des mines; nommé professêur au Jardin en 18I9.

Mémoire sur les produits volcaniques, 1 vol. in-4, 1815 . Mémoire sur les mines de houille de France, 1 vol. in-8, 1815.

Un grand nombre de mémoires dans le Journal de physique, dans le Journal des mines, dans les Annales de physique et de chimie, dans la Description de l'Égypte et dans les Annales du Muséum.

\section{Aide-naturaliste.}

M. RegLey (François-Théophile-Marie), né à Paris en 1777. Nommé au Muséum en 1812.

\section{ICONOGRAPHIE.}

M. Van-Spaendonck (Gérard), né à Tilbourg dans le Brabant hollandais, en 1746.

Membre de l'académie de peinture en I782; de l'Institut depuis la fondation; nommé suppléant de mademoiselle Basseporte au Jardin du Roi en 1774 ; titulaire en 1780 ; professeur au Muséum lors de la création de la chaire d'iconographie.

Ses tableaux sont trop connus pour que nous en parlions ici.

Il a enrichi la collection des vélins de plusieurs peintures. 
Il a fait graver une suite de dessins pour servir de modèles aux élèves : cet ouvrage est composé de six cahiers in-fol., chacun de quatre planches.

Peintres attachés au Muséum.

Pour les plantes.

M. Redouté (Pierre-Joseph), né en I $75 \mathrm{~g}$ à Saint-Hubert (Ardennes).

$C^{\prime}$ 'est d'après les dessins de cet artiste, qu'ont été exécutées les belles gravures de plantes qui accompagnent les ouvrages de l'Héritier, de Ventenat, etc. Il a beaucoup contribué à perfectionner la gravure en couleur, et il a fait lui-même de grandes entreprises d'iconographie botanique; nous nous bornerons à citer :

Les Liliacées, 8 vol. in-fol.

Planteg grasses, 2 vol. in-fol.

Les Roses, 3 vol. in-fol. et in-4.

Il y a plus de 400 dessins de lui parmi les vẻlins du $\mathbf{M u -}$ séum.

\section{Pour les reptiles et poissons.}

Redouté (Henri-Joseph), frère du précédent, né à Saint-Hubert en I 762 .

Il a fait partie de l'expédition d'Égypte, et exécuté plus de 60 dessins en tout genre pour le grand ourrage qui en est le résultat. On trouve, dans les portefeuilles du Muséum, beaucoup de dessins de lui.

Les deux frères Redouté ont été attachés au Muséum à l'époque de la nouvelle organisation. 
Pour les quadrupèdes et les oiseaux.

M. de Watluy (Pierre-François), né à Paris en r 775 ; professeur de dessin au conservatoire royal des arts et métiers.

Il a été nommé au concours peintre du Muséum, après la morl de M. Maréchal en 1803; il a continué à peindre, pour la collection des vélins, les animaux vivans dont on n'avait pas la figure, et qui lui ont été désignés par le professeur de zoologie.

Pour les vers, les insectes et les coquilles.

M. Huet (Nicolas), né à Paris en 1770 ; nommé au Muséum après la mort de $\mathbf{M}$. Oudinot, au mois d'octobre 1804 .

Outre les dessins qui ont été demandés à $\mathbf{M}$. Huet pour la partie dont il est spécialement chargé, il en a fait plusieurs de quadrupèdes et d'oiseaux. MM. Geoffroy et Cuvier lui ont aussi confié l'exécution d'un grand nombre de dessins d'anatomic.

\section{BIBLIOTHÈQQE.}

M. Toscan (Georges), né à Grenoble en i 756 ; nommé bibliothécaire au Muséum en 794 .

L'Ami de la nature, ou choix d'observations sur divers objets de la nature et de l'art, $x$ rol. in-8, 1800.

Traduction de l'ouvrage de Spallanzani, intitulé, Voyages dans les deux Siciles et dans quelques parties des Apennins, 6 rol in-8. 
$17^{6}$

HISTOIRE DU MUSÉUM.

Principal auteur de la partie d'histoire naturelle de la Décade philosophique et littéraire.

Lors de la fondation de la bibliothèque, il y avait une place de sous-bibliothécaire qui était occupée par M. Mordant de Launay. Cette place a été supprimée à sá mort en 1814 . M. Mordant de Launay est auteur d'un ourrage très-utile et très-répandu, ayant pour titre, Almanach du bon jardinier, $x$ vol. in-12. C'est lui aussi qui a fait l'enireprise de l'Herbier de l'amateur, qui se continue aujourd'hui.

MÉNAGERIE.

Garde de la ménagerie.

M. Cuvier (Frédéric), né à Montbelliard en 1773 ; inspecteur de l'académie de Paris; nommé garde de la ménagerie lors de la création de cette place, le 2 I décembre 1805 .

Histoire naturelle des mammifères, qu'il publie conjointement avec M. Geoffroy-Saint-Hilaire ; 36 livraisons chacune de 6 planches.

Des dents des mammifères, considérées comme caractères zoologiques, 1 vol. in-8, 1822.

Plusieurs Mémoires parmi ceux du Muséum.

Lazoologie des mammifères, dans le Dictionnaire des sciences naturelles.

GARDES DES GALERIES D'HISTOIRE NATURELLE.

M. Lucas (Jean-François), né au Jardin du Roi en 1747 . 
PRINCIPAUX EMPLOYES.

177

La place qu'il occupe au Muséum a été créée lors de la nouvelle oryanisation.

M. Lucas fils (Jean-André-Henri), né au Jardin du Roi en ' 780 .

Nommé adjoint de son père, le 12 février i 799 ; chevalier de la Légion d'honneur.

Tableau méthodique des espèces minérales, 2 vol. in-8, 1806-1813.

Une partie des articles relatifs à Ia minéralogie dans le Dictionnaire d'histoire naturelle de Deterville.

\section{BUREAU D'ADMINISTRATION.}

M. Thouin (Jacques), chef du bureau et caissier, né à Paris en $17^{51}$.

\section{GARDE MILITAIRE.}

La garde du Jardin est confiée à une compagnie de sous-officiers dont le commandant actuel est M. Gouvion Saint-Cyr, chevalier de l'ordre de Saint-Louis et de celui de la Légion d'honneur. 


\section{VOYAGEURS NATURALISTES.}

\section{Leschenault de ta Tour.}

Il avait été attaché, en qualité de botaniste, à l'expédition des découvertes, commandée par le capitaine Baudin. Il fit ensuite un séjour à Java, d'où il nous rapporta plusieurs objets de zoologie, et un herbier considérable.

Envoyé à Pondichéry en $181 \%$, il a parcouru une partie de la presqu'île de l'Inde et il nous a fait trois envois, dans toutes les parties de l'histoire naturelle. Le premier, en 1818 , contenait la plus riche collection de poissons et de crustacés qui fât encore arrivée au Muséum : le second de 1819 et le troisième de 1820 , présentaient beaucoup d'objets nouveaux èn zoologie et en botanique; il y avait quelques animaux vivans, au nombre desquels il faut compter le jeune éléphant qui est maintenant à la ménagerie. Chacun des enrois était accompagné de catalogues raisonnés, et de mémoires sur les productions et les cultures du pays. M. Leschenault est en ce moment à Ceylan, il doit bientôt revenir, et il nous annonce une très-riche collection.

\section{Milbert.}

Il est parti pour New-York en 1814. Il nous a, depuis cette époque, fait dix-huit envois qui ont enrichi la collection du cabinet d'un grand nombre de quadrupèdes, d'oiseaux, de reptiles et de poissons. Il nous a envoyé aussi des ani- 
maux virans qui n’avaient jamais paru à la ménagerie, et qu'on y voit aujourd'hui. Tels sont le bison et sa femelle, le grand cerf de Canada, les cerf́s de la Louisiane, l'élan d'Amérique, etc.

\section{Le Sueur.}

1 avait été attaché comme peintre d'histoire naturelle ả l'expédition des découvertes, commandée par le capitaine Baudin. Pendant le voyage il devint l'ami de Péron, dont il partagea les travaux, et il se livra entièrement à l'étude de la zoologie. Parti pour les États-Unis en 1814 , il nous a fait deux envois d'oiseaux et de poissons, et nous a adressé quelques mémoires qui sont imprimés parmi ceux du Muséum.

\section{M. de Saint-Hilame (Auguste).}

Il est parti pour Rio-Janeiro en 1816; il a parcouru plusieurs provinces du Brésil, et nous a fait quatre envois dans lesquels se trourent des oiseaux, des quadrupèdes, des coquillages, des insectes, etc. La botanique ayant été le principal objet de ses études, c'est surtout en plantes que sa collection est très-nombreuse : mais on attend son retour pour la mettre en ordre, et pour réunir son herbier à celui du Muséum. Ses mémoires sur plusieurs familles de plantes, insérés dans nos Annales avant et après son départ, prouvent qu'il possède également le talent d'observer et celui de généraliser les faits, et nous sommes persuadés qu'il fera connaître à fond la flore du Brésil.

\section{DIARD.}

1 est parti en 1816 pour les Indes orientales. Aussitôt que les affaires qui l'y avaient appelé ont été terminées, il 
s'est livré à son goat pour la zoologie et l'anatomie qu'il avait étudiées sous M. Cuvier (1). M. du Vaucel étant allé le joindre à Calcutta, ils se sont réunis pour faire des recherches d'histoire naturelle, et sont allés ensemble à Chandernagor, à Sumatra, et dans plusieurs îles de l'Archipel indien. Ils nous ont envoyé successivement le premier bouc de Cachemire qui ait paru en France, et trois collections composées d'un grand nombre d'objets nouveaux en zoologie. Ils se sont ensuite séparés pour explorer une plus grande étendue de pays. M. Diard s'est alors rendu à Java d'où il nous a fait un envoi considérable. Il est depuis parti pour la Cochinchine.

\section{Du VAuceL.}

C'est en 181 q que M. du Vaucel est parti pour le Bengale. Il y a trouvé M. Diard, auquel, comme nous venons de le dire, il s'est réuni pour contribuer aux progrès de l'histoire naturelle en enrichissant la collection du Muséum. Outre les envois que ces naturalistes nous ont faits ensemble, M. du Vaucel nous en a fait un de Sumatra, ou se sont trouvés beaucoup d'objets précienx que nous n'avions jamais pu nous procurer. Il a joint aux collections qu'il nous a fait passer, des notes et des descriptions intéressantes qu'il a adressées à $\mathbf{M}$. Cuvier. Il voyage maintenant dans les montagnes au nord-est du Bengale.

Ce n'est qu'après que MM. Diard et du Vaucel ont fait parvenir au Muséum de riches collections, que le gouvernement a cru devoir les dédommager en partie des dépenses

-(1) En 1816, M. Diard avait aidé M. Cuvier dans ses recherches sur les enveloppes du foetus, et sur les oufs des quadrupèdes. Voyez les deux Mémoires de M. Cuvier, imprimés parmi ceux du Muséum, tom. a, p. 8 a et 98 . 
VOYAGHUR NATURALISTES. I qu'ils avaient faites, et les mettre à même de poursuivre. leurs recherches en leur accordant un traitement.

M. Prée (Auguste).

Est parti en 1820 pour Saint-Thomas, d'où il est allé à la Martinique et aux Etats-Unis. Il nous a fait trois envois, relatifs à toutes les parties de l'histoire naturelle.

\section{Saupigny.}

A été envoyé au Sénégal en 1820 , par S. Exc. le ministre de la marine, qui avait demandé à l'administration du Muséum un agriculteur botaniste. Il doít bientôt revenir , et il nous annonce une collection considérable dont nous arons déjá reçu le catalogue.

\section{Fontanier.}

Voyage aux environs de la mer Noire, où il a été envoyé par S. Exc. le ministre de l'intérieur : comme il est parti depuis peu, nous n'avons pas encore reçu d'envois de lui.

Dans la Notice historique, nous avons exprimé nos regrets sur la perte que nous arons faite de MM. Havet et Godefroy, qui étaient partis à la même époque que M. Plée. 


\section{CORRESPONDANS DU MUSÉU.}

M. le baron DE Humboldt ; nommé lors de son départ pour l'Amérique, en 1798 .

Nous avons parlé dans la Notice historique des services qu'il a rendus au Muséum.

M. Bonpland, maintenant à Buenos-Ayres; nommé en même temps que $M$. de Humboldt.

A son retour du voyage qu'il avait fait avec $\mathbf{M}$. de Humboldt, il a commencé la publication des plantes qu'ils avaient recueillies ensemble, et qu'ils ont données au Muséum.

M. BaILlon, à Abbeville.

Il a enrichi la collection de zoologie de la plupart des oiseaux d'Eurgpe, et principalement des oiseaux de mer, dont il a fait une étude particulière. Il nous a fait parvenir aussi des mammifères et des poissons. Feu M. Baillon son père, autrefois correspondant du Jardin du Roi, avait envoyé un grand nombre d'objets pour la collection de zoologie, et beaucoup de notes dont Buffon a fait usage, en le citant, dans son Histoire des oiseaux.

M. Bory de Saint-Vincent; nommé lors de son départ avec le capitaine Baudin.

Il faisait partie de l'expédition des découvertes en qualité de zoologiste, mais il s'arrêta à l'île de France, d'où il alla visiter les principales îles de l'Afrique; il a inséré dans nos Annales des mémoires sur les conferves, et publié plusieurs ouvrages d'histoire naturelle. 
M. Prchon, zoologiste à Boulogne-sur-mer; nommé en 1802.

A fait plusieurs envois d'oiseaux pour le cabinet.

M. Faujas (Alexandre), fils du professeur, maintenant à la Guadeloupe; nomméen mars i 803. A envoyé des observations sur des fossiles de la Guadeloupe.

M. LÉONHARD, professeur de minéralogie à Heidelberg; nommé en 1808 .

Il a envoyé plusieurs échantillons de minéraux pour la collection. Il a publié un grand nombre d'ouvrages de minéralogie, et récemment un excellent traité de cette science.

M. Marcel de Serres, professeur de minéralogie à Montpellier; nommé en r 809 , lors de son royage en Allemagne.

Il envoya de ce pays des objets intéressans pour les collections de minéralogie et de zoologie. Il a inséré dans nos Annales plusieurs mémoires sur l'anatomie des insectes.

M. Troost (Gérard), chirurgien de la marine hollandaise; nommé en 18 ro.

A envoyé des minéraux pour le cabinet.

M. Corréa de Serra, aujourd'hui membre du conseil du roi et du conseil des finances à Lisbonne; nommé correspondant en I8 I r, lorsqu'il partit de France pour aller aux États-Unis.

Il a inséré dans nos Annales debeaux mémoires de carpologie, et envoyé de Philadelphie divers objets pour le cabinet.

M. TAunax, à Rio-Janeiro: nommé lors de son départ pour le Brésil, en 18 I5.

A envoyé une collection d'oiseaux en 1816 . 
M. SAINT-Yves, chirurgien de la marine.

$\boldsymbol{A}$ fait plusieurs envois de graines des Indes orientales.

M. LeAch, de la-société royale de Londres, conservateur des collections de zoologie au Mu- séum britannique; nommé en r818.

A fait plusieurs envois dans toutes les parties du règne animal.

M. MitchiLl, professeur d'histoirc naturelle à New-York; nommé en janvier 1821 .

A envoyé beaucoup d'objets d'histoire naturelle de l'Amérique du nord.

M. Wallich, surintendant du jardin de botanique de Calcutta; nornmé en janvier 1821 .

A envoyé beaucoup de graines et des squelettes d'animanx.

M. Mac-Leax fils, membre de la société linnéenne, à Londres; nommé en avril I $82 \mathrm{I}$.

Auteur d'un ouvrage d'entomologie : a envoyé beaucoup d'insectes et un ornithorinque conservé dans l'esprit-de-vin.

M. D'OrbignY, à la Rochelle; nommé en mai 1821 .

A fait des envois de mollusques, de crustacés et de fossiles, et inséré plusieurs mémoires parmi ceux du Muséum.

M. BuCKLAND, professeur de minéralogie et de géologie à Oxford; nommé en juin r82 I. A fait de très-beaux envois de minéraux et d'ossemens fossiles.

M. Durand de Villegégue, à la Martinique; nommé en juillet $182 \mathrm{I}$.

A envoyé des ognons de trés-belles liliacées. 


\section{COMPARAISON DE L'ÉTABLISSEMENT}

TEL QU'IL ETAIT EN I 789 , A CE QU'IL EST AUJOURD'HUI.

Avant de passer à la description des diverses parties du Muséum, nous croyons devoir présenter un résumé de ce que nous avons exposé dans la notice historique, et comparer l'établissement tel qu'il est aujourd'hui avec ce qu'il était à la mort de Buffon. Cette comparaison prouvera que la dépense qu'il exige n'est en rapport ni avec ses accroissemens ni avec les avantages qui résultent de sa nouvelle organisation, et que s'il n'était pas constitué comme. il l'est, l'enseignement des sciences naturelles serait à la fois moins complet et plus coûteux (I).

(1) Dans la comparaison que nous faisons ici des frais que coùtait autrefois le Jardin du Roi arec ceux que coûte aujourd'hui le Muséum, nous ne considérons que les dépenses annuelles ou d'entretien, et non celles qui auraient pour objet de nouvelles constructions ou des réparations extraordinaires. Ces dernières sont d'autant moins considérables, que celles d'entretien et de conservation sont mieux ordonnées et faites plus à propos. On a vu dans la notice précédente que le gouvernement avait toujours fait des sacrifices pour donner de la splendeur au Jardin du Roi, et que les acquisitions et les constructions s'étaient élevées à des sommes très-considérables pendant les seize 
Les fonds pour le Jardin du Roi, d'après les états de $\mathbf{1} 78$, étaient de. . 104,269 fr.

Il faut y joindre ceux de la ménagerie de Versailles, puisque cette ménagerie a été transportée au Muséum. Ces fonds étaient de . . . . 100,000

Total. 204,269

Les fonds fixés aujourd'hui par le budget pour l'entretien du Muséum et le paiement des employés sont de 300,00o fr. C'est moins d'un tiers en sus de ce que coûtaient autrefois le jardin et la ménagerie.

Maintenant comparons le Muséum au Jardin du Roi tel qu'il était en 1789 , sous le rapport de l'étendue, des constructions, des collections et de l'enseignement.

dernières années de la vie de Buffon. Cependant on n'avait pas obtenu. les résultats auxquels on est parvenu depuis ; l'enseignement était trèsborné, et l'on n’avait pur mettre en harmonie toutes les parties de l'établissement.

Les places qui ont été supprimées au Jardin par la nouvelle organisation, sont : $1^{\circ}$ celle d'intendant qui coûtait 12,000 fro, sans y comprendre les frais du secrétariat qui étaient de $8,000 \mathrm{fr} . ;, 2^{\circ}$ celle d'architecte, dont les appointemens fixes étaient de $1800 \mathrm{fr}$; $3^{\circ}$. celle d'un capitaine chargé de la police du Jardin, aux appointemens de 4000 fr., ayant sous ses ordres trois gardes bosquets, dont chacun recevait $600 \mathrm{fr}$. On juge que le service est fait bien mieux, et d'une manière bien plus convenable dans un établissement royal, par une compagnie de militaires, qui ne coûte point de frais extraordinaires à l'état, et pour qui le poste du Jardin est une retraite agréable. 
COMPARAISON DE L'ÉTABLISSEMENT. 187

Le Jardin du Roi contenait en 1789,43 arpens.

Le Muséum en contient aujourd'hui 79 .

Les galeries d'histoire naturelle ont été augmentées d'un étage, et la longueur en a été presque doublée. On y a joint une bibliothèque qui renferme plus de 12,000 volumes. On a construit la grande serre tempérée, deux serres chaudes, l'édifice nommé la rotonde au centre de la ménageric, et le grand bàtiment destiné à loger les animaux féroces. On a profité de deux vastes maisons réunies à l'établissement pour former avec la première les galeries de botanique, un laboratoire de zoologie et une salle d'administration; avec la seconde, le cabinet d'anatomie comparée et les laboratoires d'anẩtomie.

On a acquis des maisons pour le logement des professeurs, des aides-naturalistes, des chefs d'atelier et des principaux employés.

Ainsi le nombre des bâtimens du Muséum est avec celui des anciens bâtimens du jardin, dans le rapport de 7 à $\mathbf{r}$.

On a augmenté d'un tiers l'école de botanique, fondé les écoles de culture, des arbres fruitiers, des plantes d'usage, etarrangé dans le jardin des plantes d'ornement et plusieurs parterres. La ménagerie a été plantée d'arbres qui donnent aujourd'hui des graines pour propager les espèces utiles. Les cul- 
tures actuelles sont avec les anciennes dans le rapport de 9 à $\mathbf{I}$.

Quant aux collections :

Le nombre des plantes vivantes est doublé. Les herbiers contiennent six fois plus de plantes; la collection des fruits et autres produits du règne végétal est augmentée dans la même proportion.

La collection de quadrupèdes et d'oiseaux est vingt fois ce qu'elle ćtait. Celle des poissons, aujourd'hui la plus nombreuse qu'on connaisse, n'était presque rien auparavans. Celle d'insectes ne contenait que I 500 individus, elle en offre aujourd'hui plus de 40,000 appartenans à 22,000 espèces. Celle des coquilles s'est aussi beaucoup accrue.

La collection d'anatomie comparée, celle des ossemens fossiles et celle de géologie, si riches aujourd'hui, n'existaient pas.

La ménagerie de Versailles n'offrait qu'un petit nombre d'animaux, elle ne servait point à l'enseignement de la zoologie : celle du Muséum a vu paraître successivement plus de 500 espèces d'animaux étrangers, et nous a mis à mểme de faire des observations très-importantes.

La beauté du jardin, la grandeur des édifices, la richesse des collections, ont fait du Muséum 
COMPARAISON DE I'ÉTABLISSEMENT. I89 un monument magnifique : mais c'est l'étendue donnée à l'enseignement qui l'a vivifie et l'a rendu d'une utilité générale.

Il y avait au Jardin du Roi trois chaires, à chacune desquelles était jointe une place de démonstrateur: Il y en a treize aujourd'hui; et des aidesnaturalistes sont attachés à chacun des professeurs qui ont besoin d'être aidés dans leurs travaux. Le nombre des leçons que faisait chaque professeur était la moitié de ce qu’il est aujourd'hui. Les professeurs (deux exceptés), et les aides-naturalistes, sont logés au Muséum; ce qui les met à même de donner la plus grande partie de leur temps à l'établissement, tandis qu'autrefois plusieurs n'y venaient que pour préparer ou. pour faire leurs cours. Cette réunion leur offre encore l'avantage de communications réciproques, et c'est là ce qui a donné naissance à la collection des Mémoires du Muséum, que le Roi a bien roulu accueillir et dont il a accepté la dédicace.

Le Muséum occupe cent soixante-une personnes dont soixante-deux sont payées à l'année, et quatre-vingt-dix-neuf au mois.

Il entretient des correspondances avec tous les établissemens du même genre; il leur envoie des objets' qui peuvent servir à l'instruction, et il distribue gratuitement chaque année une pro- 
digieuse quantité de graines, de boutures et de greffes.

Si, prenant pour base les frais que coûtait le Jardin du Roi, on évaluait ceux que doit exiger l'entretien du Muséum, d'après l'étendue des objets qu'il embrasse et le nombre des emplois qui y sont établis; on supposerait que ces frais doivent être au moins quatre fois plus considérables; et cependant la dépense du Muséum ne. surpasse que d'un tiers ce que coûtaient jadis le jardin et la ménagerie du roi. Cette économie est la suite de son organisation. L'administration qui le régit est essentiellement conservatrice et prévoyante, elle distribue selon les besoins de chaque partie les fonds mis à sa disposition, elle surveille les moindres détails, elle emploie les ouvriers attachés à l'établissement pour faire les réparations au moment où elles sont nécessaires, elle ne donne rien à entreprise et fait exécuter les travaux sous ses yeux, en calculant toujours les dépenses sur les fonds qu'elle a en caisse; enfin elle rend compte au ministre de toutes ses opérations.

Il faut cependant convenir que le Muséum recevant tous les jours des animaux vivans, des arbres étrangers et des collections nouvelles dont la préparation et la conservation nécessitent des 
COMPARAISON DE L'ÉTABLISSEMENT. I frais, il peut arriver que malgré l'économie la plus sévère, une augmentation de secours soit indispensable pour prévenir des pertes qu'il serait dans la suite bien difficile de réparer : mais le gouvernement est trop éclairé pour ne pas proportionner les encouragemens qu'il accorde au Muséum à l'utilité toujours croissante de l'établissement, et aux résultats qu'il est sûr d'en obtenir.

Nous terminerons ce tableau par une considération qui ne sera pas sans intérêt aux yeux de ceux pour qui le spectacle de l'harmonie sociale et de la félicité domestique n'est pas moins touchant que celui de la nature. Au milieu de l'agitation d'une grande ville c'est vraiment une belle chose qu'un établissement où sont réunies cinquante familles vivant en paix, occupées de travaux utiles, contentes de leur sort, attachées au lieu qu'elles habitent pour la vie, et s'enorgueillissant de sa prospérité, soumises volontairement à une hiérarchie qui maintient l'ordre sans blesser l'amour-propre, étrangères aux rivalités de professions comme aux dissensions politiques, et bénissant à la fois le gouvernement qui les protége et l'administration qui les régit. Les savans qui se livrent aux recherches les plus difficiles, aux théories les plus élevées pour pénétrer les secrets 
192 HISTOIRE DU MUSE UM.

de la nature, rapprochent d'eux les ouvriers, et ceux-ci s'éclairant par le reflet des connaissances qui les environnent, jouissent du résultat des travaux qu'on leur fait exécuter. Tous concourent au même but, et c'est ici que se trouvent ensemble les deux sources de bonheur dont parle Virgile

Felix qui potuit rerum cognoscere causas. : .

Fortunatus et ille deos qui novit agrestes. 


\section{DESCRIPTION DU MUSÉUM.}

\section{CHAPITRE PREMIER.}

JARDIN, ÉCOLES, SERRES.

§. PROMENADE AU JARDIN.

Les personnes qui désirent voir successivement toutes les parties du Jardin, sans repasser plusieurs fois dans le même endroit, doivent suivre une marche régulière que nous allons indiquer.

Nous supposons qu'on arrive au Jardin par la porte ouverte sur le quai au milieu de la place demi-circulaire qui est vis-à-vis du pont. En entrant on embrasse d'un coup d'œil l'ensemble de l'établissement. En face et à l'autre extrémité, on voit le cabinet d'histoire naturelle qui occupe toute la largeur du Jardin, et paraît s'élever audessus de deux enclos dont l'un est la pépinière, et l'autre un vaste bassin carré, creusé en pente jusqu'au niveau de la rivière et garni d'arbrisseaux sur les talus. A droite et à gauche sont les deux grandes allées de tilleuls, et plus loin, d'un côté la ménagerie, qui s'étend jusqu'à la rue de Seine; de l'autre des plantations qui vont jusqu'à 
I94 DESCRIPTION DU MUSÉUM.

la rue de Buffon. On tournera à droite, on entrera dans la grande allée, et l'on arrivera à l'entrée de la cour qui est devant le cabinet. En suivant la grille de fer qui entoure cette cour, l'on se trouvera au commencement de l'allée de tilleuls parallèle à celle qu'on vient de quitter. On aura derrière soi le cabinet, et un peu à droite la maison nommée autrefois l'intendance, et qui fut le logement de M. de Buffon depuis $\mathbf{I}_{77^{3}}$ jusqu'à sa mort ( $\mathrm{r}$ ) : ce sera là notre point de départ. Nous allons faire le tour du Jardin pour examiner tout ce qui se trouvera sur notre passage; d'abord dans la partie basse qui s'étend du cabinet au bord de la rivière, puis dans la partie élevée qui se compose de deux collines dont l'une porte le nom de labyrinthe, et l'autre celui de petite butte. Nous irons ensuite parcourir la ménagerie que nous considérerons seulement sous le rapport des sites et des plantations, et nous reviendrons sur le quai par l'allée des marroniers, qui sépare le jardin des semis et la ménagerie de la partie plate du Jardin. Cette promenade exigerait à peu près une heure si on ne s'arrêtait nulle part: elle sera beaucoup plus longue; il faudra même la renouveler plusieurs fois, si l'on veut donner quelque attention à ce qu'elle

(1) Cette maison est habitée aujourd'hui par MM. de Lamarck et Fan-Spaendonck, prof'esseurs s et par MM. Lucas, gardes des galeries. 
-offre de plus instructif et de plus curieux. Nous ne parlerons point en ce moment des parties du jardin qui ont une destination spéciale, comme l'école de botanique, celle de culture, celle des arbres fruitiers, le jardin des semis, les serres, et la ménagerie, considérée comme l'habitation des animaux. Chacun de ces établissemens doit être l'objet d'une visite particulière.

Nous voici donc au haut de la grande allée de tilleuls plantée par M. de Buffon en 1740 , sur la lisière méridionale du jardin, parallèlement à celle par laquelle nous sommes arrivés. En suivant cette allée jusqu'au bout, nous aurons à droite, des plantations et un parc clos destiné à diverses cultures; à gauche, deux parterres séparés par un grand bassin, puis la pépinière, le bassin carré garni d'arbrisseaux, enfin d'autres parterres. Ne nous occupons point de ce qui est à notre gauche, et que nous verrons au retour, mais seulement de ce qui est entre lâllée et la rue de Buffon.

Les quatre premiers carrés entre les parterres et cette rue, sont composés d'arbres de toute espèce et de tout pays qui peuvent passer l'hiver en pleine terre. Ceux qui sont plus rares ou plus intéressans, sont répétés plusieurs fois. On y remarque entre autres un gleditzia ou févier sans épines, envoyé en 1748 du Canada par M. de la Galissonnière, et qui est l'un des plus grands ar- 
Ig6 DESCRIPTION DU MUSÉUM.

bres de la plantation; un sophora du Japon, Ie premier qui ait été cultivé en Europe, où il a pendant long-temps été fort rare (I); le premier acacia venu de l'Amérique septentrionale, que Vespasien Robin qui en était possesseur, planta dans le Jardin du Roi en 1635 : cet arbre avait encore il y a quelques années plus de soixante pieds d'élévation, mais les branches supérieures s'étant successivement desséchées, on a été obligé de le recéper, pour qu'il repoussât du tronc. C'est de cet individu que sont venues les graines qui onl commencé à répandre en France 1'un des arbres les plus agréables et les plus utiles; et c'est en mémoire du service rendu par Robin, qui l'a cultivé le premier en Europe, que Linnzeus lui a donné le nom de Robinia. On y voyait autrefois un superbe marronier planté en $\mathbf{1 6 5 6}$, lors qu’il n’en existait encore qu'un seul en France. Nous en faisons mention parce que la récolte de ses graines a produit la plupart des individus de la même espèce qui font aujourd'hui la décoration de nos parcs. Il a péri dans l'hiver de 1766 à г767.

Les deux carrés qui sont vis-à-vis et un peu au-

(1) Les graines en furent envoyées a B. de Jussieu par le P. d'Incarville en 1747. Il fleurit pour la première fois en France en 1779. Jusqu'alors on l'avait nommé Arborincognita Sinarum. Il pousse avec beaucoup de vigueur et de rapidité, et réussit même dans les terrains pierreux. Il s'élève de 60 à 80 pieds; son bois est fort dur : les Chinois tirent de ses fleurs une belle couleur jaune. 
dessous du bassin étaient l'écolè des arbres et arbrisseaux du temps de Tournefort. Lorsque l'école de botanique a été replantée sous M. de Buffon par M. de Jussieu, on y a pris les arbres qu'on ne pouvait se procurer facilement ailleurs, pour les mettre à leur place, et on en a substitué d'autres à ceux qu'on a enlevés. Parmi ceux qui restent on trouve encore un ordre qui rappelle la méthode de Tournefort. On doit y remarquer surtout un genevrier (juniperus excelsa, Marsch.), qui a quarante pieds de haut, et quinze pieds jusqu'à la naissance des branches; il fut apporté du Levant et planté au jardin par Tournefort, et c'est probablement le seul qui existe en France. Nous n'avons que l'individu mâle. Ses feuilles froissées entre les doigts, répandent une odeur très-forte.

A l'extrémité de ce carré, et dans le local même où se terminait l'école des arbres de Tournefort, se trouve un café, autour duquel sont des siéges et de petites tables pour ceux qui veulent prendre quelques rafraîchissemens en se reposant à l'ombre des arbres.

Au-dessous du café sont trois carrés entourés d'un treillage en bois. Du vivant de M. de Buffon le terrain qu'ils occupent était un taillis qu'on avait négligé de cultiver, et c'est seulement depuis quelques années que M. Jean Thouin l'a mis. dans l'état où on le voit aujourd'hui. 
Le premier de ces trois carrés est destiné à la culture des plantes annuelles, qui à cause de la beauté de leurs fleurs, sont recherchées pour l'ornement des jardins; elles y sont séparées par petits compartimens dont chacun est occupé par une espèce, ctl'ensemble du parterre est lui-même divisé en quatre parties où les diverses espèces sont placées selon la saison dans laquelle elles fleurissent. Les graines de ces fleurs sont recueillies avec soin pour faire des distributions et répandre ainsi des plantes agréables.

Le second carré est également destiné aux fleurs d'ornement, mais on n'y cultive que des plantes vivaces. Vers la fin de l'automne on donne aux amateurs des racines ou des rejetons de celles qui peuvent être multipliées de cette manière. On prend souvent dans l'un et l'autre de ces deux parterres des modèles pour le cours de dessin de M. Van-Spaendonck (I).

Le troisième carré est destiné au semis des arbres et arbustes de pleine terre; qu'on repique ensuite dans la pépinière, et dont on se sert pour garnir le jardin. On y voit un très-joli massif du pêcher d'Ispahan, dont M. Olivier apporta des noyaux à son retour de Perse en 1780 , et dont

(1) On ne cultive point au Muséum les fleurs doubles, les jacinthes, les primevères, etc.; parce qu'on ne vent pas nuire au commerce des iardiniers fleuristes. 
M. Thouin a donné l'histoire dans le huitième volume des Annales du Muséum.

A l'extrémité de ce carré se trouve une allée transversale de tulipiers de Virginie, qui fait la limite de l'ancien jardin. A partir de ce point, les tilleuls de la grande allée sont moins élevés, parce qu'ils n'ont été plantés qu'en I 783 (r).

Viennent ensuite quatre carrés, dont chacun a une destination particulière. Le premier, clos d'un treillage, est consacré aux arbres qui conservent leur verdure toute l'année. Ces arbres sont plantés par gradation, les plus grands au nord le long de l'allée, les autres au midi du côté de la rue de Buffon, pour que tous jouissent du soleil autant que cela est possible. On y voit de très-grands épicéa, le pin de Jérusalem (pinus alepensis, Desf. Fl. Atl.), de beaux genevriers de Virginic ou cédres rouges, un chêne aux glands doux (quercus Ballota, Desf. Fl. Atl.) (2), des houx panachés, le houx de Mahon, etc.

Ce carré est séparé du suivant par une allée de

(1) Le tulipier (liriodendrum tulipifera) a été introduit en France vers 1748 par le marquis de la Galissonnière, gouverneur du Ganada. C'est un des plus beaux arbres de l'Amerique septentrionale; dans les forêts oủ il croît naturellement il s'élève à cent trente pieds et domine les autres, arbres; son tronc a quelquefois plus de vingt pieds de circonférence. Les tullipiers du Jardin ont été plantés il $y$ a vingt-cinq ans par M. Thouin, ils se convrent de fleurs au mois de juin, et depuis quelques années ils donnent des graines fertiles.

(2) En Espagne on mange ces glands comme dés chataignes. 
mélèzes (I); celui-ci également entouré d'un treillage, contient un assortiment des arbres dont on fait des bosquets d'automne. Ce sont ceux dont les feuilles ou les fruits se colorent pendant cette saison. Là se trouve le noyer pacanier (juglans olivaformis, Willd.) (2), le plus grand qui existe en France; deux gingko biloba (3), les premiers arrivés dans nos climats, et dont un seul a donné des.fleurs mâles; un beau diospyros ou plaqueminier de Virginie; un mespylus linearis qui forme naturellement le parasol (4); un superbe mûrier rouge de l'Amérique septentrionale, dont les fruits sont aussi bons que ceux du mûrier noir, et qui est surtout remarquable en ce que son feuillage, plus touffu que celui d'aucun autre arbre

(1) Les mélèzes s'élèvent à cent vingt pieds sur les montagnes, dans les terrains frais et ombragés, mais ils ne réussissent pas au Jardin du Roi. Les mélèzes et le cyprès chauve, sont les seuls arbres de la famille des coniféres qui perdent leurs feuilles pendant l'hiver.

(2) Get arbre, originaire de la Haute-Louisiane, croît abondamment le long des rivières et dans les maxais. Ses noix sont un objet de commerce aux États-Unis; il n’a pas encore fructifié en Francé.

(3) Grand arbre du Japon très-singulier par son feuillage; ses fruits gros comme des pommes, contiennent une amande qu'on sert sur les tables, c'est ce qui l'a fait nommer aussi noyer du Japon. Il fut introduit en Angleterre en 1754 et apporté en France quelques années après par M. Petigny.

(4) Cet arbre, originaire de l'Amérique septentrionale, n'était pas connu des botanistes lorsque les graines ont été pportées au Jardin il y a environ vingt-cinq ans. On l'a multiplié, on en a formé une petite allée, et il sera bientôt répandu dans les parcs. Son port est entièrement différent de celui des autres espèces du même genre. 
que nous connaissions, se conserve jusqu'à la fin de l'automne, et n'est jamais attaqué par les insectes.

Ce carré se termine par une allée d'érables à fruit cotonneux (acer eriocarpon, Mich.), arbre qui n'est bien connu que depuis que M. Desfontaines l'a distingué de ses congénères en le décrivant dans les Annales du Muséum, tom. 7 .

Le carré suivant est un bosquet d'arbres d'ornement pour l'été. Les plus grands sont toujours au nord, on a cherché à faire contraster entre eux ceux qui diffèrent le plus par le port, par le feuillage, par la forme et la couleur des fleurs. On y remarque des frênes de Caroline, des frênes à fleurs, des noyers noirs de Virginie, des chicots (grmnocladus canadensis) (I). Cette plantation est terminée par une allée d'aylantes (2). Tous por-

(1) Cet arbre est dioïque : il est remarquable par ses feuilles deux fois ailées qui ont jusqu'à trois pieds de long sur vingt pouces de large; en hiver lorsqu'il en est dépouillé, ses branches étant en petit nombre, if a l'aspect d'un arbre mort; c'est ce qui lui a fait donner le nom de chicot. Nous n'avons encore que l'individu mâle.

(2) Les graines de cet arbre furent envoyées de la Chine par le P. d'Incarville en 1751 . M. Desfontaines l'ayant vu fructifier pour la première fois chez $\mathbf{M}$. Lemonnier à Versailles, le reconnut pour un nouveau genre de la famille des térébinthes, et il en publia la description dans les Mémoires de l'académie des sciences en 1786; il lui donna le nom d'aylante qu'il porte à Amboine, et qui signifie arbre du ciel; on l'avait d'abord désigné sous le nom de vernis du Japon, parce $q u^{3}$ on avait cru que les Japonais en tiraient leur beau vernis. Ciette dénomination s'est conservée, quoique très-impropre; elle conviendrait mieux au rhus vernix, Linn. 
tent d'un côté, sur le tronc, l'impression de la gelée dont ils furent frappés en 1789 , à l'époque où la séve avait commencé à se mettre en mouvement.

Le dernier carré est planté d'arbres qui fleurissent au printemps : son étendue était double avant la construction du pont; on futalors obligé d'en retrancher une partie pour agrandir le quai. Les arbres qu'on enleva furent transportés vis-àvis, à l'extrémité de la grande allée correspondante. Parmi ceux qui restent de ce côté se trouvent le le pavia jaune, le pavia de l'Ohio, et le marronier à fleurs rouges, espèce très-remarquable et qui n'était point encore connue il y a deux ans, lorsqu'elle a fleuri au Jardin du Roi.

Arrivés à l'extrémité de ce bosquet, nous nous détournerons un peu de notre route pour en visiter la seconde partie, également composée d'arbres du printemps, greffés de différentes manières et abandonnés à leur croissance naturelle. On y voit des merisiers à fleurs doubles; un bel individu du coignassier de la Chine, décrit et figuré par M. Thouin dans les Annales du Muséum, tom. I9, lorsqu'il a fructifié pour la première fois en 18 I I le pommier odorant (malus coronaria) de l'Amérique septentrionale, et le pommier à bouquets (malus spectabilis) de la Chine. Ce bosquet ^st défendu de la poussière par un brisant formé 
de thuyas de la Chine. Il est borné du côté de l'ouest par une allée d'arbres de Judée, qui est la plus jolie du Jardin, lorsqu'au commencement de mai, les feuilles n'étant pas encore développées, toutes les branches sont couvertes d'une innombrable quantité de fleurs.

Revenons maintenant sur la terrasse jusqu'à la porte d'entrée. Nous trouverons en face de cette porte une allée qui va jusqu'au grand bassin carré, et à droite et à gauche de cette allée, des parterres employés à diverses cultures. Ces parterres sont au nombre de huit. Les quatre premiers sont consacrés à la culture des plantes médicinales, dont on fait des distributions gratuites aux pauvres. Elles y sont disposées par bandes, et toutes étiquetées, pour que les herboristes et même les étudians en pharmacie et en médecine puissent les examiner dans tous leurs développemens. Deux de ces carrés sont destinés aux plantes indigènes : les deux autres aux plantes exotiques. On y voit les différentes espèces de rhubarbe, et plusieurs plantes nouvellement introduites, auxquelles on attribue de grandes vertus dans les pays d'où elles viennent, et dont les médecins pourront fairel'essai chez nous avec les précauțions convenables.

Les deux parterres suivans, contiennent des doubles des plus belles plantes vivaces de l'école. Comme elles ont plus d'espace, elles peuvent y 
croître avec plus de liberté, et les élèves en botánique viennent les étudier, et s'exercer à les déterminer d'après les caractères. C'est pour cela qu'on n'y met point d'étiquettes.

Les deux derniers carrés sont un parterre où l'on cultive des fleurs de plate-bande. On a soin de varier les espèces toutes les années, et l'on renouvelle les plantations deux fois pour que le terrain soit également couvert de fleurs depuis le milieu du printemps jusqu'au milieu de l'automne. Cette partie du Jardin offre un coup d'œil agréable. Les graines qu'on y recueille font partie des distributions.

A l'extrémité de ces parterres on trouve le bassin carré qui est entouré d'une grille de fer'; en en faisant le tour, on peut voir tous les arbrisseaux dont il est planté. Ces arbrisseaux sont disposés sur les côtés en amphithéâtre, à quatre expositions différentes selon leur nature: Au printemps, et jusqu'à la fin de l'été, ce bassin offre de tous côtés un coup d'œil magnifique, par la quantité de rosiers, de boules de neige, de lilas, de fustets, de fontanésia (r), de staphylea qui le décorent. Plusieurs des arbrisseaux de cette plantation commençant à se dégrader, on l'a coupée

(1) Joli axbrisseau décrit et dédié a M. Desfontaines par M. de la Billardière, qui en a apporté les graines au Jardin du Roi, à son retour de Syrie en 2788. 
l'hiver dernier, à rez de terre, ainsi on ne la trouvera pas aussi belle qu'elle l'était en i 820 ; mais elle le sera beaucoup plus dans les années suivantes. Au fond est un petit étang qui reçoit par infiltration les eaux de la rivière. On avait d'abord destiné ce bassin à la culture des plantes aquatiques; mais elles n'ont pu y réussir. Il serait à désirer qu'on pût creuser un aquéduc qui établît entre ce bassin et la Seine une communication plus facile, ou qu'on se décidât à combler le fond, où l'eau stagnante et non renouvelée devient souvent fétide pendant les grandes chaleurs. On a établi vers le bas, des plates-bandes où l'on cultive des fleurs d'ornement (I).

Après avoir faitle tour de ce carré, on traverse une allée bordée d'un seul côté de mespilus linearis et de koelreutéria (2). Ces deux arbres placés alternativement sur une même ligne, contrastent par leur feuillage, par leurs flcurs, et surtout par

(1) Lorsqu'on construira de nouvelles serres, on les placera probablement à la suite de celles qui existent, ce qui obligera de prendre une lisière de terrain dans la partie occidentale de l'école de botanique, et conséquemment de prolonger cette école du côté du midi audelà de ses limites actuelles; alors on comblerait le grand bassin pour y transporter l'école des arbres fruitiers ou celle de culture. Cette translation exigeant des travaux préliminaires, il est possible qu'on s'en occupe le printemps prochain, et que le grand bassin ne réponde plus à la description que nous en avons donnée.

(2) Joli arbre originaire du nord de $\mathrm{l}^{2}$ Asie, introduit en France en $27^{8} 8$, et acclimaté dans le jardin des semis du Muséum, doù il s'est, répandu chez les amateurs. 
206 DESCRIPTION DU MUSÉUM.

le port, l'un ayant les branches étendues horizontalement, et l'autre les ayant réunies en boule. On entre ensuite dans la pépinière, qui est entourée d'une grille de fer.

C'est là qu'on élève les arbres, arbrisseaux et arbustes nécessaires pour garnir les différentes parties du Jardin : on y propage de bouture, de marcotte, ou de greffe, toutes les espèces intéressantes qui sont nouvellement introduites ou qui ne sont pas encore répandues dans le commerce, et l'on en donne de jeunes pieds aux cultivateurs qui sont en correspondance avec le Muséum.

Parmi les arbrisseaux élevés à la pépinière on voit avec plaisir le charmant marronier apporté de l'Amérique septentrionale par A. Michaux, (cesculus macrostachya, Mich. Fl.) qui étale ses branches et ses longues grappes de fleurs à trois pieds au-dessus de la terre. On y voit aussi une plantation de paliurus (1) aussi beaux que ceux qui croissent dans le midi de la France; des cissus, des périploca. Au reste ces cultures varient, parce que chaque année on transplante ailleurs les végétaux qui sont assez multipliés, pour leur en substituer d'autres. Au milieu de la pépinière est un rucher

(1) Joli arbrisseau d'ornement dont on peut faire des haies impénétrables, parce qu'a l'aisselle de chaque feuille il y a deux épines dont l'une est droite et l'autre courbe. Virgile en fait mention : spinis paliurus acutis. On le nomme vulgairement porte-chapeau à cause de ses fruits qui ont la forme d'un chapeau rabattu. 
composé de trente-six ruches, réunies dans un petit pavillon formé d'une charpente en bois, et couvert d'une toile peinte en rouge. Ces ruches sont de diverses constructions, depuis les paniers les plus simples jusqu'aux ruches à feuillets et à celles qui sont garnies en verre pour qu'on puisse voir travailler les abeilles. M. Lasseray qui prend soin de ce rucher, y a fait des observations sur les divers moyens de recueillir le miel sans détruire les mouches; il a même trouvé celui de l'extraire des alvéoles sans altérer les gâteaux de cire.

Le long de la grille du côté du midi, est une plate-bande couverte de terreau de bruyère, et ombragée par les tilleuls de la grande allée. Elle est destinée aux plantes qui exigent de la fraîcheur et des soins particuliers; c'est là qu'on cultive plusieurs arbustes de la famille des bruyères ou des rosages; des azalea, des kalmia, des vaccinium, des rhododendron, des itea, des andromeda, dont iln'est pas possible d'avoir des individus aussi beaux dans l'école de botanique. On y remarque aussi un joli magnolia glauca, l'un des arbrisseaux les plus recherchés à cause du parfum de ses fleurs.

En sortant de la pépinière on se trouve en face de deux parterres, plantés il y a quelques années sous le ministère de $\mathbf{M}$. Chaptal, qui accorda au Muséum les fonds nécessaires pour cette dépense. 
Ces deux parterres, entourés d'un treillage, sont employés à la culture, à la multiplication et à là naturalisation des plantes étrangères vivaces et de pleine terre. On y voit une ligne du beau phlomis apporté du Levant par Tournefort, une de diverses espèces de férule dont la tige a dix pieds d'élévation, une de fraxinelles, une de l'aletris d'Abissinie, que l'on a multiplié au Jardin, plusieurs espèces de phlox, deux cheloné nouvellement venus du Mexique ( $C$. campanulata et $C$. barbata, etc.). On y voit aussi vers le bas une collection de rosiers à fleurs doubles, qui, sans être aussi nombreuse que celle du Luxembourg, offre cependant les espèces les plus remarquables. Sur les glacis sont des touffes de plantes bulbeuses ou tubéreuses, comme des pivoines, des couronnes impériales, des lis orangés et des martagons de diverses couleurs, des albuca, des a maryllis, etc.

Les planches sont entourées de plantes propres aux bordures que l'on varie d'une année à l'autre, pour les faire connaître eț remarquer. Plusieurs des plantes qu'on y a ainsi cultivées pendant deux ans se sont introduites depuis dans les jardins. Ainsi le joli silène apporté de Barbarie par M. Desfontaines, forme maintenant des bordures charmantes dans les parterres du Luxembourg.

Dans la belle saison, on place aux extrémités de ces deux carrés et dans l'intervalle qui les sé- 
pare, des arbres en caisse qu'on a retirés de 1'orangerie : tels que des grewia, des lentisques, le cassia corymbosa, le célastrus à fleurs nombreuses, des justicia, le kiggelaria d'Afrique, le caroubier, le sterculia à feuilles de platane, ou tong-chu des Chinois; et du côté du cabinet, des myrtes à fleur double, de magnifiques lauriersroses et des palmistes.

Dans l'intervalle qui sépare ces deux parterres se trouve un large bassin où l'on cultive plusieurs plantes aquatiques, dont quelques-unes sont étrangères, telles que le saururus cernuus et le jussiea à fleurs jaunes jussicea grandiflora, Mich. ), tous deux de l'Amérique septentrionale. Sur' le bord du bassin qui est entouré d'un treillage, on cultive quelques plantes qui aiment l'humidité, et surtout de très-jolies saxifrages.

La'construction de ce bassin est extrêmement singulière; il a la forme d'une coupe portée sur un pied, et l'on en fait le tour en dessous. On pourrait y placer quelques plantes cryptogames qui se développent dans l'obscurité. Les pierres en sont si bien liées, que sous ce chapiteau il y a une humidité continuelle, mais sans que l'eau s'échappe d'aucun côté.

Les carrés dont nous venons de parler sont en face d'un jardin particulier entouré d'une grille de fer; c'est celui de l'orangerie, où l'on place 
dans des caisses ou des pots pendant la belle saison toutes les plantes qui, exigeant moins de chaleur que celles de la serre tempérée, ont passé l'hiver dans l'orangerie. On y remarque de beaux yuccas, des phormium ou lin de la Nouvelle-Zélande (1), le laurier amandé du Mississipi, le prunier toujours vert, un magnolier à grandes fleurs, des casuarina (2), des clethra, des plantes du midi de la France, et beaucoup de plantes des Alpes.

Au fond de ce jardin est le bâtiment de l'orañgerie dont les murs sont couverts de plantes grimpantes et surtout de la belle bignone de Virginie, qui attire les yenx par ses yrands bouquets de fleurs rouges.

(1) Le phormium tenax, avec lequel les habitans de la Nouvelle-Zélande fabriquent leurs étoffes, a du rapport avec les aloès. Il est connu depuis le voyage du capitaine Cook. Le Muséum en ayant reçu quelques pieds au retour de l'expédition du capitaine Baudin en donna un à M. Faujas, qui l’ayant multiplié dans son jardin près de Montelimar, le répandit dans les départemens du midi. On le cultive aujourd'hui dans le voisinage de la mer où il réussit fort bien. C'est une acquisition précieuse pour la marine; parce que les. corrles faites avec les fihres de ses feuilles ont une force double de celles de chanvre. Voyez sur la culture et les usages de cette plante les Annales du Muséum, tome 2 et tome 17 .

(2) Les casuarina ou filao appartiennent a la famille des conifères. Ils sont entièrement dépourvus de feuilles, comme les éphédra. Leurs rameaux grêles, pendans et articulés, leur donnent un aspect tout particulier. On pourrait les cultiver en pleine terre dans le midi de la France. Leur bois très-dur, très-agréablement veiné et susceptible d'un beau poli , serait fort utile dans l'ébénisterie. C'est à la Nouvelle-Hollande celui qu'on emploie de préférence pour la construction des vaisseaux. 
Cette orangerie n'a point de fourneau. Elle est divisée en deux parties dont une seulement est voûtée: les croisées sont de la largeur des trumeaux; onles couvre de paillassons dans les grands froids. Au printemps on voit fleurir les plantes alpines sur l'appui des croisées. Ces plantes, quoiqu'elles soient originaires des régions les plus froides, ne peuvent chez nous passer l'hiver en pleine terre, parce qu'elles n'y sont pas, comme dans leur pays natal, couvertes par la neige depuis les premières gelées jusqu'au retour de la belle saison.

A côté de l'orangerie est un très-petit enclos bien abrité du nord et de l'ouest, où sont des couches et des châssis. On y place quelques plantes délicates qu'on veut faire fleurir ou multiplier de bouture.

En sortant du jardin de l'orangerie, on est à côté de la rampe qui conduit au haut du jardin, c'est-à-dire à la colline qu'on nomme le labyrinthe, et à une autre butte moins élevée.

La colline à laquelle on donne vulgairement le nom de labyrinthe, parce qu'elle est coupée par plusieurs chemins qui rentrent les uns dans les autres, est d'une forme conique. En y montant on trouve d'abord le cédre du Iiban, qui donne toutes les années des graines en quantité. C'est le premier qui ait paru en France. Lorsque 
Bernard de Jussieu alla en Angleterre avec du Fay en 1734 , Collinson, médecin quarke, fort riche et amateur de botanique, avait reçu du Liban un cône de cédre dont les graines avaient levé. Il en donna à Bernard de Jussieu deux individus qui n'avaient que quelques pouces de haut. Ils furent cultivés avec soin : l'un des deux fut placé dans l'ancienne école, et n'existe plus; l'autre est celui qui étend majestueusement ses branches au pied du labyrinthe. C'est de ses graines que sont venus plusieurs de ceux qui sont maintenant répandus dans les parcs. Quoiqu'il soit d'une très-grande hauteur, il serait encore plus élevé si sa flèche n'avait été cassée par un accident: on sait que ces sortes d'arbres' poussent par le sommet des branches, et que lorsque le sommet est coupé ils ne croissent plus.

Au-dessous du cédre du côté du midi sont deux pins à pignons remarquables par leur grandeur, et qui donnent l'idée de l'effet que font ces arbres sur les Apennins dont ils couronnent les cimes, et dans les jardins d'Italie, où ils sont cultivés. II y a aussi plusieurs laricio, des pins du Lord, des hemlock-spruce, le baumier de Giléad, beaucoup d'ifs, d'épicéa, de cyprès, et de très-beaux genevriers de Virginie.

En suivant les allées qui montent en spirale et font plusieurs fois le tour de la colline, on trouve 



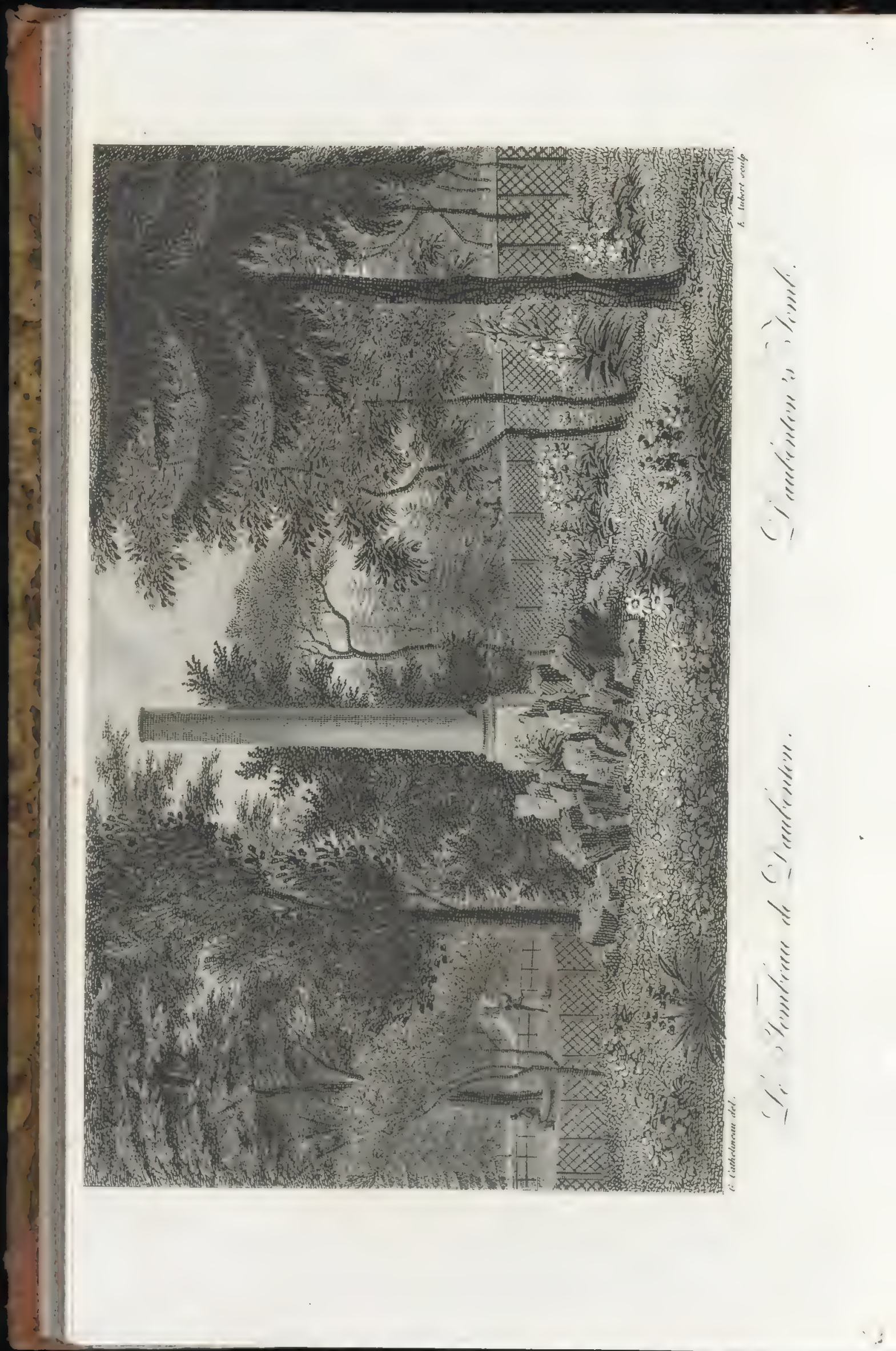


au sommet un joli kiosque entouré de colonnes de bronze et d'une balustrade. De ce point élevé la vue s'étend sur le Jardin, sur toute la partie de l'est, du sud et du nord de Paris, et sur les campagnes éloignées du côté de Montmartre, de Vincennes et de Sceaux.

A mi-côte à l'exposition du levant, entre le kiosque et le cédre, on voit une petite enceinte formée par un treillage : c'est là qu'est le tombeau de Daubenton. Ce patriarche de l'histoire naturelle étant mort au Muséum le 3i décembre I799, on crut devoir conserver ses cendres dans le lieu où il avait passé sa vie, et auquelil avait rendu de si grands services. Une colonne posée sur divers minéraux, autour desquels sont des fleurs qu'on a soin de renouveler, indique ce monument. On a le projet de placer sur cette colonne le buste en marbre de Daubenton.

En descendant de la butte, du côté qui incline vers le nord; on voit un bel érable de Montpellier; au-dessous, le plus beau platane qui existe à Paris; et entre ces deux arbres, sur le penchant du coteau, un châlet où l'on trouve le matin, pendant la belle saison, du laitage et des oeufs frais. C'est là que plusieurs de ceux qui viennent passer la matinée à étudier au Jardin vont faire des collations agrestes en respirant sous l'ombrage des arbres un air frais et balsamique: Au-dessous. du 
2 I4 DESCRIPTION DU IIUSEUTM.

châlet est un chemin qui fait le tour de la colline, se prolonge le long d'une terrasse élevée au-dessus de la rue du Jardin du Roi, et aboulità une porte de sortie des galeries d'histoire naturelle:

En revenant à l'est derrière les serres, on sera de nouveau conduit au-dessous des pins à piznon dont nous avons parlé, et l'on se trouvera en face de la petite butte.

Celle-ci forme un carré long, presque en amphithéâtre. Elle est coupée par des allées sinueuses; elle est, comme la précédente, plantée en arbres verts, parmi lesqueis on remarque plusieurs espèces de pins, particulièrement celui d'Alep, des sapinettes noires et rouges, des cédres du Liban, des chênes verts, des filaria, de beaux individus du buis de Mahon, un néflier du Japon, un petit massif d'aucuba japonica. Le haut de cette butte forme une esplanade à l'extrémité de laquelle est un point de vue très-pittoresque, qui s'étend sur tout le Jardin jusqu'à la rivière.

Les deux collines dont on vient de parler sont couvertes d'herbe qu'on fauche toutes les années. On y trouve souvent plusieurs plantes étrangères qui viennent des graines de celles qu'on a semées dans l'école, et qui s'y naturalisent. Ainsi nous y avons vu pendant plusieurs années le seigle de Crète, le maceron du Levant, smyrmium perfoliatum, etc. 
En descendant de la petite butte, on a en face un grand ovale qui est placé devant l'amphithéâtre et entouré d'un treillage : à gauche quelques logemens de professeurs, dont chacun a un petit jardin particulier; et plus loin, la porte qui donne sur la rue de Seine : à droite, le jardin des semis, la serre tempérée et la ménagerie.

Ce grand ovale est destiné à recevoir pendant l'été les plus beaux arbres de la Nouvelle-Hollande, du cap de Bonne-Espérance, de l'Asie mineure, des côtes de Barbarie, qui ont passé l'hiver dans la serre tempérée. Ils y sont groupés par masses pittoresques, et les caisses qui les contiennent reposent sur un gazon où l'on place de distance en distance diverses fleurs d'ornement. Ces arbres étant étiquetés nous n'avons pas besoin d'en indiquer le nom. Nous ferons mention des plus remarquables en visitant la serre tempérée où ils passent l'hiver. Nous nous bornerons à dire en ce moment, qu'on ne peut voir nulle part une telle réunion d'arbres étrangers de différente forme et d'un aspect aussi varié.

Au milieu de l'ovale est une très-grande coupe de pierre, autour de laquelle on arrange les arbres les plus élevés, et sur laquelle on place ordinairement dans un vase une plante remarquable par la beauté et l'éclat de ses fleurs.

A côté de la porte de l'amphithéâtre on voit 
deux beaux palmistes (I) qui furent envoyés Louis XIV par le margrave de Bade-Dourlach, au commencement du siècle dernier. Ils avaientalors douze pieds de tige; ils ont actuellement vingt-cing picds. Comme ces arbres croissent par le sommet et non par des bourgeons latéraux, qu'il ne se forme pas de nouvelles couches sur le tronc, et qu'ils poussent chaque année de nouvelles feuilles, tandis que les plus anciennes tombent; le nombre des anneaux qui se voient sur la tige indique leur âge, comme les couches concentriques du bois indiquent celui des arbres à deux feuilles séminales.

On est obligé de retenir ces palmistes par quatre cordes qui aboutissent au-dessous du șommet, et sont attachées aux quatre angles de la caisse pour qu'ils ne soient pas cassés par le vent.

En Sicile et en Espagne on n'en rencontre jamais qui soient d'une aussi grande élévation. Il est remarquable que cet arbre croît par la base au-dessous de la colonne, comme par le sommet, tellement que l'impression du premier anneau de feuilles qui était d'abord au niveau de la terre, en est aujourd'hui à plus de deux pieds de distance (2).

(1) Chamaerops humilis, L., on le nomme aussi palmier éventail.

(2) Ge genre d'accroissement paraît d'abord très-singulier, et nous n'en connaissons pas d'autre exemple; cependant il ne s'écarte point 
Nous allons maintenant continuer notre promenade en parcourant la ménagerie, que nous ne considérerons point comme le lieu où l'on élève divers animaux, nous proposant d'y revenir ensuite pour cet objet.

On entre dans la ménagerie par une porte voisine de l'amphithéâtre. A mesure qu'on avance, la diversité des sites, la différente inclinaison des parcs, la variété des plantations, le grand nombre de fabriques curieuses donnent à cette partie de l'établissement l'aspect d'un jardin irrégulier ou jardin anglais. Mais ici l'intérêt est tout autre. Dans la plupart des jardins anglais, où l'on n'a eu d'autre intention que de produire des effets pittoresques et de varier les points de vue, on se demande souvent si les effets obtenus répondent aux frais qu'on a été obligé de faire pour donner du mouvement au terrain. On se lasse bientôt de ce qui n'offre rien d'utile. Ici tout a un but déterminé : les parcs nombreux qui divisent la ménagerie étant destinés à renfermer chacun une es-

des lois ordinaires de la végétation. Le pivot de la racine ayant étét repoussé hors de terre par les racines inférieures qui ne pouvaient s'enfoncer, s'est élevé et accrú les petites racines qui y adhéraient se sont successivement détruites en rendant sa surface raboteuse, et ce même pivot forme aujourd'hui la base ou piédestal de la colonne du palmier. Une chose digne d'attention, c'est que près de la terre il y a un étranglement considérable, comme si l'arbre n'avait plus aujourd'hui la même force de végétation, et n'était soutenu que pax des racines partant du centre. 
pèce d'animalł̣leur grandeur est relative aubesoin que cet animal a de faire plus ou moins d'exercice; les arbres sont disposés de manière à lui fournir de l'ombrage; les fabriques rappellent les habitudes de l'animal auquel elles servent de retraite, et le pays d'où il est originaire. Les inclinaisons du terrain sont calculées pour que ceux qui veulent examiner ou dessiner les animaux puissent les voir de tous les côtés, de bas en haut, dans toutes les positions, et bien éclairés à toutes les heures du jour. Les bassins sont destinés aux oiseaux aquatiques; un petit ruisseau renouvelle l'eau dans laquelle ils se baignent. Le grand édifice placé au centre renferme les quadrupèdes qui doivent être chauffés pendant l'hiver, et les femelles qui ont des petits qu'elles allaitent et qui ont besoin de soins particuliers. La faisanderie, bien abritée du nord, a devant elle un terrain découvert. Les loges des animaux carnassiers, construites l'année dernière sur un plan très-simple et très-beau, sont exposées au midi, et situées dans le lieu le plus voisin de la rivière, afin qu'on puisse avoir aisément l'eau nécessaire pour les laver, et que cette eau puisse s'écouler par un aquéduc souterrain. Nous reviendrons sur cet objet. Nous avons dû en dire un mot ici pour qu'on n'attribue point à un vain caprice la variété qu'on remarque dans ce beau Jardin. 
Lorsqu'on est entré dans la ménagerie, il convient de prendre aliernativement les allées à gauche et à droite. On fait ainsi le tour de divers parcs; on passe devant les singes, les oiseaux de proic et la faisanderie, et l'on se trouve vis-à-vis de la rotonde. Alors on avance au delà de cet édifice, on tourne à gauche pour visiter de nouvelles constructions; puis reprenant la direction qu'on avait d'abord suivie, on arrive au bâtiment qui renferme les animaux carnassiers, et l'on revient en passant entre d'autres parcs et en suivant la terrasse qui aboutit à la serre tempérée. Au-dessous de cette terrasse est le jardin de naturalisation qui tient à celui des semis, et qu'on voit parfaitement de ce point. Quand on est parvenu à l'extrémité de la serre tempérée, on descend dans le lieu le plus bas, et l'on revient par un chemin creux au lieu d'où l'on est parti.

En faisant le tour des parcs, on aura pu remarquer que chacun d'eux est entouré d'un treillage qui diffère des autres par le dessin. On aura surtout été frappé de la beauté et de la variété des arbres qui ombragent les allées.

C'est dans cette partie de l'établissement qu'on a rassemblé tous les arbres et tous les arbrisseaux étrangers qui peuvent passer l'hirer en pleine terre. Nulle part un aussi grand nombre d'espèces parvenues à leur. yrandeur naturelle, ne se trouve 
dans un espace aussi resserré. Comme le terrain est bon, et que les arbres ont été bien soignés, ils ont pris un accroissement rapide.

Ces arbres n'ont été plantés que successivement depuis I797, et l'on a lieu de s'étonner de. leur grandeur. Il est bon d'avertir que la plupart ont été plantés lorsqu'ils avaient déjà acquis une trèshaute taille, qu'on ne les a point étêtés, et qu'on n'en a même retranché qu'un petit nombre de branches, afin de ne point altérer leur port naturel. On a eu soin, en les arrachant en hiver, de n'en point endommager les racines, de conserver même la motte autant qu'on l'a pu, de bien défoncer et de bien préparer le terrain, de les orienter comme ils l'étaient dans le lieu d'où on Jes a retirés, de les arroser au besoin pendant le premier été; et ils se sont à peine ressentis de la transplantation. C'est une preuve de l'inutilité de couper la tête aux arbres qu'on veut transplanter; et M. Thouin, qui s'est élevé contre cette pratique dans ses cours et dans ses écrits, a donné ici la preuve la plus concluante de la vérité de ses principes.

Les arbres de la ménagerie étant étiquetés, nous n'avons pas besoin d'en donner la nomenclature. Nous nous contenterons de signaler quelques-uns de ceux qui doivent attirer l'attention, soit parce qu'ils sont très-rares, soit parce qu'ils sont ici d'une beauté remarquable. 
On y roit le premier mûrier à papier arrivé en France. M. Banks l'avait apporté d'Otaïti, et il l'envoya au Jardin du Roi. Il a été long - temps dans le jardin des semis près du puits; mais comme ily donnait beaucoup d'ombre, on l'a transplanté dans la ménagerie. Le noyer noir et le noyer cendré (I) qui se couvrent de fruits toutes les années, les diverses espèces de micocouliers, les frênes et les érables d'Amérique, les sorbiers de Laponie, le tilleul argenté, les cerisiers et les pruniers de l'Amérique septentrionale, le poirier à feuilles de saule, les sumacs, les féviers sont ici d'une grandeur remarquable. L'acacia visqueux que Michaux a apporté d'Amérique, et qui est si agréable par son feuillage et par ses fleurs roses qui se renouvellent deux fois l'année, est très-multiplié ;

(1) Juglans nigra. Cet arbre croît en plein bois dans les états du Kentucky et de l'Ohio, il $\mathrm{y}$ acquiert jusqu'à soixante-dix pieds d'élévation, et six pieds de diamètre. Son bois est d'une couleur très-brune, mais il est compacte et susceptible d'nn beau poli : il ne se tourmente point et n'est jamais attaqué par les vers, On en fait de beaux meubles, et on l'emploie dans les constructions navales. C'est un des arbres qu'il serait le plus utile de multiplier en France; il pousse plus vite et s'élève plus rapidement que le noyer commun, auquel il est préférable excepté pour ses fruits. M. Michaux fils propose d'en faire des pépinières, et de greffer les sujets à huit ou dix pieds de bauteur, avec notre noyer. Alors, dit-il, on jouirait de tous les avantages que présentent - les deux espèces, sous le rapport de la qualité du bois et de la bonté $\approx$ des fruits. Histoire des arbres de l'Amérique septentrionale, tom. 3 , p. 164 .

Le noyer cendré est aussi un fort bel arbre, mais il est à tous égards inféricur ał noyer noir. 
et ce sont les individus élevés d'abord au Jardin du Roi qui ont produit ceux qui sont aujourd'hui répandus dans les parcs.

La plupart des arbres de la ménagerie fructifient abondamment. Ils sont ce qu'on appelle des porte-graines, c'est-à-dire, destinés à fournir la récolte des graines qu'on distribue toutes les années aux cultivateurs. I.es arbres verts de la famille des conifères sont les seuls qui ne se trouvent point à la ménagerie, parce qu'on les a tous réunis sur les buttes.

On voyait, il y a quelques années, le long des allées qui séparent les parcs, plusieurs arbustes agréables, des spiréa, des chèvre-feuilles, des clématites, etc ; ils y sont plus rares aujourd'hui, parce que les arbres devenus trop grands, les privent du soleil.

Il y a encore dans la ménagerie quelques carrés qui ne sont pas plantés d'arbres; ils seront bientôt ombragés comme les autres. On en a profité jusqu'ici pour multiplier quelques plantes rares, ou pour faire des expériences : ainsi il y a quelques années qu'on y a cultivé le pastel, pour savoir quelle quantité d'indigo on pourrait en retirer.

Autour des divers parcs, on place des arbustes ou des plantes vivaces qui donnent de belles fleurs, des iris, des millepertuis, etc.

En sortant de la ménagerie, on tourne à gauche, 
et l'on passe devant le chemin qui la sépare de la serre tempérée. Au milieu de ce chemin est un puits qui, à l'aide d'une pompe, fournit de l'eau pour remplir les bassins. Les chameaux du Muséum sont continuellement employés à mettre cette pompe en mouvement.

En suivant la terrasse le long de la serre et de la grille occidentale du jardin des semis, on se trouve en face de l'école de botanique, et si l'on veut réserver pour une seconde promenade les autres parties de l'établissement et rejoindre la porte par laquelle on est entré, on suit l'allée des marroniers jusqu'à la terrasse qui est sur le quai. En la suivant, on a à gauche le jardin des semis et celui de naturalisation, puis trois larges fossés ou cours pavées, où l'on place des animaux qui s'y promènent penđant le jour, et se retirent la nuit dans des loges construites sur le côté. On a vu pendant plusieurs années des ours de Berne dans les deux premiers: les tours qu'on leur faisait faire amusaient beaucoup le public; mais un homme atteint de folie s'étant "jeté dans le fossé et ayant été tué, on a cru devoir loger ces ours ailleurs. Le troisième de ces fossés renferme des sangliers qui ont plusieurs fois soulevé et amoncelé les grosses pierres du pavé. A l'extrémité de ces cours sont les parcs de la ménagerie; enfin l'emplacement des loges où l'on renfermait 
224 DESCRIPTION DU MUSÉUM.

les animaux carnassiers avant la construction du bâtiment qu'îls occupent aujourd'hui. A droite on trouve successivement l'école de botanique, une allée de sophora da Japon, entre lesquels on a mis des thuya, qu'on supprimera lorsque les sophora seront parvenus à leur grandeur; l'école des arbres fruitiers, l'allée des platanes, l'école des plantes d'usage dans l'économie rurale et domestique, l'allée des catalpas de Virginie, l'école de culture, l'allée d'arbres de Judée; enfin, la portion du bosquet du printemps dont nous avons parlé.

Nous allons maintenant nous occuper de ces diverses parties. Nous parlerons d'abord de l'école de botanique, puis de celle des arbres fruitiers, de celle des plantes d'usage, de celle de culture, du jardin des semis ; enfin de la serre tempérée et des serres chaudes. Chacun de ces objets exige un article à part. Après avoir vu tout ce qui est relatif au Jardin, nous examinerons les collections renfermées dans les cabinets, la ménagerie et la bibliothèque. 
§ II. ÉCOLE DE BOTANIQUE.

Cetre école entourée d'une grille de fer, s'étend de l'ouest à l'est depuis la rampe qui monte de la partie basse à la partie haute du jardin, jusqu'au carré planté d'arbres fruitiers. Elle est bornée au nord par la serre et par l'allée des marronniers, au sud par l'allée des tilleuls. Sa surface est de 3,064 toises carrées. Des allées longitudinales et transversales la partagent en seize compartimens, qui comprennent cent cinquante-quatre plates-bandes d'inégale grandeur, mais qui ont en général 60 pieds de long sur 5 pieds de large, et sont séparées les unes des autres par des sentiers de 3 pieds. Dans l'allée du milieu, qui est la plus large, sont quatre bassins placés à égale distance, et dont l'eau sert aux arrosemens. Des cinq portes de cet enclos deux sont toujours ouvertes, excepté à l'heure où les jardiniers vont prendre leur repas: cependant on n'y voit jamais que des personnes occupées; le public sent que c’est un lieu réservé pour l'étude.

On a soin d'en éloigner les enfans, non-seulement à cause du dégât qu'ils pourraient faire, mais surtout à cause du danger auquel ils se- 
226 DESCRIPTION DU MUSÉUM.

raient exposés s'ils touchaient à quelques plantes vénéneuses.

L'école de botanique est la partie essentielle et fondamentale du jardin : c'est elle qui établit une correspondance entre toutes les autres parties consacrées à la culture, en fixant d'une manière positive la nomenclature des végétaux et les rapports des espèces. C'est elle qui constate l'état actuel de nos richesses en botanique, et qui assure la propagation de la science en mettant les élèves à même d'étudier les plantes dans tous les périodes de leur accroissement, de les comparer entre elles et avec les descriptions qui en ont été données, et d'observer les changemens qu'elles éprouvent et les phénomènes physiologiques qu'elles présentent selon les saisons, l'heure du jour, l'intensité de la lumière et l'état de l'atmosphère. C'est elle enfin qui facilite le moyen de recueillir successivement les graines à l'époque de leur maturité, et de les étiqueter sans avoir à craindre une méprise, lors même que l'espèce qui les fournit étant dépouillée de ses feuilles, ne peut être distinguée de l'espèce voisine.

Mais les avantages qu'elle présente et les résultats qu'elle produit disparaîtraient bien vite, si le même esprit qui a présidé à sa formation ne présidait à son entretien. Il faut que le professeur qui en a la direction connaisse assez bien 
les plantes pour les distinguer aussitôt qu'elles ont développé leurs feuilles, pour assigner à chacune la place qui lui convient, pour rectifier les erreurs que commettent fréquemment ceux dont on reçoit des envois, pour rapprocher les divers noms donnés à la même plante par différens botanistes, pour séparer les espèces qui ont été confondues sous le même nom et qu'il est nécessaire de distinguer par un nom nouveau; enfin pour décrire et publier celles qui sont entièrement inconnues, ou qui n'ont encore paru dans aucun jardin de l'Europe et dont il n'existe ni description ni figure. Il faut encore qu'il ait une surveillance continuelle : car, malgré le soin qu'on prend pour la récolte des graines, on voit souvent d'autres plantes pousser à la place de celles qu'on avait semées, quelquefois des étiquettes sont déplacées par accident, et la confusion serait bientôt introduite dans l'école, si le professeur ne la visitait tous les jours, et s'il n'était frappé au premier coup d'oeil de ce qui dérange l'ordre qu'il a établi (I).

Ce n'est pas seulement dans le temps des cours que l'école de botanique est fréquentée par de

(1) Il est nécessaire que le professeur de botanique et le jardinier en chef soient secondés par un jardinier qui aime les plantes et qui sache les soigner. Cette place est remplie avec beaucoup de zèle et d'intelligence par M. Matthieu qui a été formé par M. Jean Thouin, ct chargé pendant plusieurs années du soin de la serre tempérée. 
nombreux étudians. Aussitôt que la floraison dè la perce-neige et du bois gentil annonce le réveil de la végétation, elle est visitée par ceux qui veulent faire de la botanique une étude méthodique, et chaque jour, quelques fleurs nouvellement épanouies, leur offrent le sujet de nouvelles observations. C'est là, et d'après les leçons du professeur actuel, que se sont instruits plusieurs botanistes aujourd'hui très-connus. Ils y ont appris à analyser les organes et à distinguer les caractères sur les plantes vivantes, et se sont ainsi rendus habiles à reconnaitre dans les herbiers ceux des plantes du même genre qui ne sont point encore parvenues dans les jardins. Car ni les plantes desséchées, ni les meilleures figures, ne peuvent suppléer à l'étude de la nature vivante.

Il existe peut-être ailleurs une collection de plantes aussi nombreuse que celle du Jardin du Roi, surtout si l'on met en ligne de compte les variétés qu'on prend pour des espèces lorsqu'elles sont isolées. Mais il n'existe sûrement dans aucun pays une réunion de végétaux disposés selon l'ordre de leurs rapports naturels, où l'on puisse voir en même temps les plantes de tous les climats dont les graines ont pu lever en Europe. En comparant la liste des plantes qui y sont cultivées, avec les Species publiés depuis linné, on voit ce qui nous manque encore; on distingue ce qui est bien 
connu de ce qui ne l'est pas, on est à même d'adresser dans tous les pays des demandes pour compléter la collection autant qu'il est possible.

Cette école n'est cependant pas très-ancienne, et elle n'eût pu être ni aussi riche ni ordonnée suivant le même plan, avant que la science eût fait les progrès qu'elle doit aux travaux de Tournefort, à la nomenclature de Linné, aux familles naturelles de Jussieu. Elle ne fut d'abord qu'une collection de plantes médicinales, la plupart indigènes. Au retour des voyages de Tournefort, elle s'enrichit de quelques plantes étrangères, mais elle n'allait que jusqu'à l'extrémité du mur de la serre adossée à la petite butte; et quoiqu'on en eût écarté les arbres et les arbrisseaux, on ne pouvait y placer toutes les plantes. Ce fut Buffon qui, à la sollicitation de M. de Jussieu, en tripla l'étendue, et l'entoura d'une grille en $\mathbf{1 7 7 4}$, et les végétaux y furent alors disposés dans un ordre régulier. Cependant le local se trouva bịentôt trop resserré, et elle fut encore augmentée d'un quart en 1788 , lorsque Buffon eut acquis les terrains qui la bornaient du côté de l'est. En I802, M. Desfontaines la replanta de nouveau, après avoir recherché toutes les espèces qui, étant arrivées depuis peu, se trouvaient dispersées dans les parterres, les serres et le jardin des semis, ete 
s'en être procuré d'autres qu'il savait exister cher quelques amateurs.

Le Jardin du Roi possède aujourd'hui environ six mille cinq cents espèces de plantes. Celles qui périssent sont remplacées par des acquisitions nouvelles, et comme on en reçoit beaucoup plus qu'on n'en perd, le nombre en augmente chaque année.

Toutes ces plantes ne peuvent être placées constamment dans l'école de botanique; quelquesunes sont trop délicates pour qu'on les expose en plein air, d'autres sont trop rares pour qu'on ne les garde pas avec précaution lorsqu'on n'en a qu'un seul individu. Mais s'il en est un certain nombre qui ne peuvent rester à l'école, toutes y sont portées les jours de leçon, lorsque le professeur traite de la famille à laquelle elles appartiennent.

Les plantes annuelles ne se voient pas toujours à l'école, parce qu'il en est plusieurs dont la durée est très-courte : on tâche cependant de remédier à cet inconvénient en les semant dans diverses saisons, ce qui fait qu'on les y trouve quelquefois lorqu'elles ne sont plus dans les champs. Il est aussi des plantes qui croissent naturellement dans les marais, dans les bois, ou sur les coteaux, et qui se montrent rebelles à la culture et périssent promptement dans les jardins ; telles 
sont plusieurs pédiculaires et plusieurs orchidées. On a soin de les renouveler lorsqu'elles disparaissent ; un jardinier étant chargé d'aller les recueillir à la campagne.

Toutes les plantes sontétiquetées: desétiquettes plus grandes et de couleur rouge indiquent d'abord les classes et les familles; ensuite vient l'étiquette dugenre qui est placée au-dessus de celle de la première espèce du genre; enfin les étiquettes des espèces, dont chacune porte sur la première ligne la lettre initiale du genre et le nom classique latin, sur la seconde le nom français, et sur une troisième l'indication du pays où la plante croît naturellement, et des signes qui marquent si elle est annuelle, vivace ou ligneuse, si elle est de pleine terre, ou d'orangerie, ou de serre. Au-dessous de ces signes on voit, sur plusieurs étiquettes, une bande colorée en rouge ou en vert, ou en jaune, ou en bleu, ou en noir, et destinée à indiquer si la plante est d'usage dans la médecine, dans l'économie domestique ou dans les arts, si elle est recherchée pour l'ornement des jardins, si elle est vénéneuse.

L'emploi de ces bandes colorées épargne beaucoup de travail aux personnes qui veulent examiner un certain ordre de végétaux d'après un point de vue particulier. Par exemple, ceux qui font des recherches sur les plantes médicinales, 
ou sur les plantes d'ornement, peuvent se dispenser de s'arrêter à toutes les espèces, et ne fixer leur attention que sur celles qui les intéressent spécialement, et qui leur sont désignées par la couleur de la bande tracée sur l'étiquette.

Ainsi l'on peut, en fréquentant l'école, étudier successivement, et sous plusieurs rapports, toutes les familles et tous les genres dont quelques individussont arrivés en Europe. Un catalogue faitpar M. Desfontaines, et qui a eu deux éditions, offre la liste de celles qui y sont cultivées, et fait connaître la synonymie de celles qui ont été décrites sous différens noms. Les plantes qui ne paraissent à l'école que les jours de leçon, étant indiquées par l'étiquette qui est à leur place; ceux qui désireraient les étudier à l'époque de la floraison, peuvent demander au professeur de botanique ou au jardinier en chef de les leur faire voir.

On rencontre en quelques endroits des plantes cultivées sur le milieu de la plate-bande, et sans étiquette. Ce sont celles qu'on a nouvellement reçues et dont on n'a pu déterminer l'espèce parce qu'elles n'ont pas encore fleuri, quoique leur port ait suffi pour en faire connaître la famille et même pour indiquer le genre auquel elles doivent appartenir.

Pour suivre les plantes de l'école, on entre par la porte de l'ouest située au bas de la rampe, et 
l'on détourneà droite. Les premières plates-bandes s'étendent le long de la grille qui est parallèle à la grande allée. Les plantes y sont disposées sur deux lignes, à la distance convenable. Elles sont rangées selon la méthode de M. de Jussieu, dans laquelle les premiers ordres sont ceux des champignons, deś algues et des mousses: on a supprimé ces familles qui ne peuvent être cultivées, et l'on a commencé par les fougères dont on possède plus de cinquante espèces (I); viennent ensuite les näiades, les aroïdes, les souchets et les graminées, qui commencent vers l'extrémité et forment la seconde ligne en remontant jusqu'au point d'où l'on est parti. Le nombre des espèces rangées sur les deux lignes que nous venons de suivre est d'environ cinq cent quarante, dont trois cent dix graminées. La plupart de ces plantes craignant la chaleur, elles se trouvent bien d'être ombragées par les arbres de la grande allée.

En quittant cette plate-bande, on tourne à droite et l'on revient à l'extrémité ouest. Dans l'angle qui est au-dessous de la serre de du Fay, commencent les palmiers. On suit toutes les allées qui séparent les plates - bandes en avançant de

(1) Plusieurs des fougères qui se plaisent dans les lieux humides et ombragés ne se conservent pas long-temps à l'école, et comme il est très-difficile d'en faire lever les graines, on nia pas constamment toutes celles qui sont portées sur le catalogue. 
234 DESGRIPTION DU MUSÉ M.

gauche à droite jusqu'au bas de l'école. Les plantes sont sur deux rangs, dans toutes les familles où il y a peu d'arbres; dans celles où les végétaux exigent un plus grand développement et doivent passer l'hiver en pleine terre, comme dans les érables, les rosacées, les amentacées, les conifères, elles occupent le milieu de la plate-bande.

Des espèces de cages ouvertes d'un côté, sont placées sur les plantes qu'il est nécessaire de garantir soit du vent du nord, soit des rayons du soleil, et des cloches de verre sur celles de la zone torride qui exigent une chaleur concentrée.

Les plantes aquatiques, comme les näiades, les nymphéa, quelques renoncules, sont dans des baquets remplis d'eau qu'on renouvelle à mesure qu'elle s'évapore; celles qui se plaisent sur les pierres, comme plusieurs fougères et plusieurs joubarbes, sont placées sur un sol artificiel semblable à celui où elles croissent naturellement.

Après les palmiers, dont nous avons douze à quinze espèces, viennent les joncs, les liliacées, parmi lesquelles sont les aloès, puis les iridées , les orchidées et les hydrocharidées où l'on voit le vallisneria, plante aquatique, célèbre, non par sa beauté, mais par la manière dont s'opère chez elle la fécondation ( $\mathrm{r}$ ). Ici finissent les monocoty-

(1) Le Rhône impétueux, sous son onde écumante, Durant dix mois entiers, nous dérobe une plante 
Jédons; leur nombre est au Jardin de onze cents. Nous faisons cette remarque, parce que cette proportion est la même qu'on trouve en comparant, depuis les fougères jusqu'aux arbres verts, le nombre des monocotylédons à celui des dicotylédons dans l'ensemble des végétaux connus sur le globe.

La série des dicotylédons commence par les aristoloches, qui forment une classe particulière. On trouve ensuite les chalefs, les protées, les lauriers, les polygonées où sont les rhubarbes, puis les amaranthes, les labiées, et les solanées, dans lesquelles le seul genre solanum comprend plus de soixante espèces. Là se trouvent aussi des datura et des tabacs qui ont récemment été apportés. Plus loin, et vers le tiers de la longueur de l'école, on a pratiqué une banquette garnie de terreau de bruyère, entourée d'un treillage ct

\footnotetext{
Dont la tige s'allonge en la saison d'amour, Monte au-dessus des flots, et brille aux yeax du jour;

Les males, jusqu'alor's dans le fond inmobiles,

De leurs liens trop courts brisent les nouds débiles,

Voguent vers leur amante, et libres dans leurs feux,

Lui forment sur le fleuve uni cortége nombreux:

On dirait d'une fête où le dieu d'hyménée

Promène sur les flots sa pompe fortunée :

Mais les temps de Vénus une fois accomplis,

La tige se retire en rapprochant ses plis,

Et va mûrir sous l'eau sa semence féconde.
}

Castex, poëme des Plantes, chant $1^{\text {er. }}$ 
236 DESCRIPTION DU MUSÉU.

abritée du côté de l'ouest et du midi, pour les plantes de la famille des bruyères dont la culture exige des soins particuliers.

On arrive ensuite à la grande famille des composées dont les espèces cultivées au Jardin sont au nombre de huit cent cinquante. Presque toutes y sont de la plus belle végétation, et se couvrent de fleurs, surtout en automne. Il n'en est pas de même des rubiacées: cette famille si considérable entre les tropiques, ne présente qu'un petit nombre d'espèces qui, comme le gardenia, l'houstonia, le café, etc., puissent être bien cultivées dans les serres. Vers le milieu de l'école, après les géranium, les malvacées et les magnoliers, et aù point où commencent les caryophyllées, on voit une tonnelle garnie de plantes grimpantes : elle couvre une partie du passage souterrain qui conduit au jardin des conches. Plus loin sont les inyrtöidesqui, sur quarante-cinq espèces, en présentent trente arrivées depuis peu de la NouvelleHollande, et recherchées pour la décoration des jardins; les rosacées auxquelles appartiennent nos plus belles fleurs et nos fruits les plus savoureux; les légumineuses dont nous avons six cents espèces, parmi lesquelles plusicurs du genre dessensitives, ne nous sont connues que depuis le dernier royage du capitaine Baudin; les térébinthes, famille singulière composée d'arbres et d'arbris- 
seaux qui se ressemblent tous par l'odeur forte de leurs feuilles; là se trouve la nọix d'acajou, le pistachier, le manguier, et les sumacs, genre dans lequel nous devons signaler deux espèces, le rhus toxicodendron et le rhus radicans, dont le suc est si caustique, qu'une seule goutte tombée sur la peau suffit pour causer une inflammation qui s'étend bientôt à toute la surface du corps. Non loin de ces plantes ligneuses se présentent, rampant sur la terre ou grimpant sur un treillage, des plantes entièrement différentes de celles qui les précèdent et de celles qui les suivent, et par leur physionomie et par leurs propriétés.: ce sont les cucurbitacées et les passiflores. La série des végétaux de l'école est terminée par les amentacées et les arbres verts; deux familles qui réunissent les plus yrands arbres de nos forêts.

Sur la dernière plate-bande, on voit quelques plantes, comme le sarracenia, le bégonia et le coriaria ou redoul, dont la place n'est pas encore bien fixée dans l'ordre naturel.

Nous ne nous arrêterons point à indiquer les plantes de l'école qu'on ne pourrait trouver ailleurs, parce que toutes sont mentionnées dans le Catalogue de M. Desfontaines. Nous nous bornerons à dire qu'on y voit un superbe liquidambar d'Orient, un bel assortiment de gleditzia, parmi lesquels se trouve celui de la mer Caspienne, un 
beau virgilia (I) dont nous devons la connaissance ct l'introduction à M. Michaux fils, et un mimosa julibrissin ou arbre de soie, qui appelle les regards par la délicatesse de son feuillage, la couleur et l'élégance de ses houppes de fleurs. Vis-à-vis du troisième bassin, et parmi les légumineuses, se trouve un beau pin lariccio, qui était à sa place lorsque M. Desfontaines a agrandi l'école, et qu'on n'a pas osé transplanter. Ces grands arbres sont d'un effet pittoresque ; ils interrompent la monotonie des plates-bandes. Près d'une des portes qui donne sur l'allée des tilleuls, il y a deux rosiers greffés sur églantier dont la tête se couvre de fleurs de plusieurs espèces à quinze pieds au-dessus du sol.

On supprime de l'école les variétés, à moins qu'elles ne soient remarquables et constantes. Comme elles sont très-nombreuses, elles auraient pris un terrain et exigé des soins qui doivent êtrè employés à conserver les types primitifs des espèces.

Dans la belle saison, lorsqu'on a retiré les plantes de la serre chaude, on les arrange le long du mur de cette même serre à l'exposition du

(1) Les bourgeons de cet arbre, comme ceux du platane, sont xenfermés dans le pétiole de la feuille et ne se montrent qu'après la chute de celle-ci. C'est à cause de la couleur jaune de son bois que M. Michaux l'a nommé virgilia lutea. Voy. Hist. des arbres de l'Amér. sept., tom. 3, pag. 266. 
midi, sur une banquette qui n'est séparée de l'école que par un treillage de deux pieds de hauteur. Elles y sont disposées de manière que les plus petites sont sur le devant et n'empêchent point de voir celles qui sont plus élevées. Un échantillon de chacune d'elles est porté à côté de l'étiquette, à la place qui lui appartient dans l'école (I).

Les démonstrations de botanique se faisaient autrefois à l'école; mais le nombre des élèves étant devenu très-considérable, il était impossible qu'ils y fussent placés convenablement. La plupart ne pouvaient entendre, quelques-uns seulement pouvaient voir. On se précipitait sur les plates-bandes et l'on courait risque de faire beaucoup de dégât. Aujourd'hui le cours se fait à l'amphithéâtre; le professeur y porte un échantillon des espèces dont il veut parler, et après la leçon, les élèves viennent eux-mêmes observer les plantes en place.

La botanique est une science si vaste, qu'il n'est pas possible de l'apprendre dans une année. Ceux qu'un goût particulier détermine à y consacrer tous leurs loisirs, et qui se proposent' d'en posséder un jour l'ensemble, feront bien de s'instruire

(1) La banquette sur laquelle on doit arranger pendant l'été les plantes de la serre, sera moins étendue en 1822 qu'elle ne l'était les années précédentes, parce qu’on a été obligé de prendre un tiers de sa longueur pour l'emplacement de la nouvelle serre construite pendant l'automne de 1821 . Au printemps prochain on cherchera les moyens de suppléex à ce défaut d'espace. 
240 DESCRIPTION DU MUSEUM.

avec méthode et sans embrasser trop d'objets à la fois. Pendant la première année qu’ils suivront le cours, ils doivent se borner à étudier dans l'école, après chaque leçon, les genres dont le professeur a parlé, en vérifiant sur plusieurs espèces les caractères de la fructification. L’année suivante, ils viendront aussitôt que la végétation commence, et ils examineront toutes les plantes à mesure qu'elles fleurissent, et lorsque leurs graines commencent à mûrir.

Les amateurs qui, sans prétendre approfondix les détails de la science, voudront prendre une idée générale des phénomènes de la végétation et des rapports qui existent entre les végétaux dispersés sur le globe, visiteront l'école avec le plus grand intérêt depuis le commencement du printemps jusqu'à la fin de l'automne. Ils seront frappés de voir chaque plante placée à côté de celle qui lui ressemble le plus, tellement que, sauf quelques interuptions et quelques lacunes, on passe par degrés des liliacées, comme la tulipe et la jacinthe, aux conifères, comme le sapin et le cédre du Liban. Ils remarqueront quelques familles si naturelles, que l'homme le moins habitué à observer saisit au premier coup d'oeil les analogies qui en rapprochent les espèces; telles sont les liliacées, les labiées, les crucifères; d'autres où les genres sont réunis par des caractères moins 


$$
\text { ECOLE DE BOTANIQUE. }
$$

apparens, comme les nerpruns, les euphorbes, etc. Ils verront des familles nombreuses, comme les composées et les papilionacées, d'autres qui sont bornées à un petit nombre d'espèces, comme les aristoloches et les millepertuis; quelques - unes qui se nuancent avec leurs voisines, comme les crucifères avec les capparidées; d'autres qui s'en séparent brusquement, comme les ombellifères des renoncules, les euphorbes des cucurbitacées; ils y verront enfin quelques plantes dont le caractère est si tranché, que la place qu'on leur a assignée dans la série des végétaux, n’a été déterrninée que par des considérations systématiques : telles sont le réséda, la capucine et le parnassia. Ils y observeront aussi des genres dont les espèces se ressemblent asse' pour qu'on ne saisît pas leur différence si on les trouvait isolées, et qu'il est cependant utile de distinguer. Ainsi les asters sont à l'école au nombre de soixante espèces qui se nuancent; mais ils fleurissent à diverses époques : la plupart sont recherchés pour l'ornement des jardins; et c'est leur port et le temps de la floraison qui doit déterminer le choix. Ainsi beaucoup de graminées qu'on ne distingue que par des caractères minutieux, offrent de grandes différences par la rapidicé de leur croissance, ce qui fait préférer les unes aux autres pour les prairies artificielles. 
242 DESCRIPTION DU MUSEU.

En parcourant l'école dans diverses saisons, on y voit se succéder les fleurs depuis le daphné jusqu'à l'espèce d'hellébore appelée rose de Noël: et le mois de janvier est le seul où l'on ne puisse trouver des fleurs que dans l'orangerie.

Le rapprochement de toutes les plantes dans un même local, met à même d'observer quelques phénomènes très-curieux : tel est celui de l'épanouissement d'un grand nombre de fleurs à une heure déterminée, les unes s'ouvrant au lever de l'aurore, comme plusieurs cistes; d'autres à midi, comme certains ficoïdes; d'autres au coucher du soleil, comme la belle-de-nuit, les onagres, etc. Quelques-unes même; amnoncent le temps qu'il doit faire, comme le souci pluvial et le laitron de Sibérie, dont le premier s'ouvre et le second se ferme le matin lorsqu'il doit faire beau dans la journée.

Le sommeil des feuilles qui se présente sous des formes si différentes, non-seulement entre les plantes de diverses familles, comme les légumineuses et les malvacées, mais encore dans des genres voisins, comme les sensitives et les casses, et dans les diverses espèces d'un même genre, comme les astragales, ne peut être bien observé qu'autant qu'un très-grand nombre d'espèces sont rapprochées dans le même lieu et à la même exposition. Et cependant ces phénomènes sont d'au- 
lant plus intéressans et fournissent des caractères d'autant plus essentiels, qu'ils tiennent à la vie des régétaux.

Il en est un peut-être plus curieux encore et qu'on ne peut voir également qu’à l'école de botanique : c'est que plusieurs fleurs répandent leur parfum après le coucher du soleil, comme le geranium triste, le gladiolus tristis, le cestrum noc. turnum, quelques giroflées, etc. ; et que presque toutes celles qui ont cette singulière propriété, l'annoncent par leur couleur qui est un mélange de jaune, de rougeâtre et de blanc (I).

Chaque année en parcourant I'école on y remarquera quelque plante nouvellement introduite, et qui doit un jour ajouter de l'agrément à nos parterres. Il serait trop long d'énumérer ici celles quisont sorties du jardin de Paris. Nous nous bornerons à faire observer que des arbrisseaux qui portent des fleurs éclatantes, comme le camellia japonica, et les plantes employées à la décoration des jardins dans les pays étrangers, comme l'anthemis ou camomille à grandes fleurs (2),

(1) Voy. Mémoire sur les Crucifères, par M. Decandolle; danś les Mém. du Mus., tom. 7, pag. 184.

(2) La camomille à grandes fleurs, vulgairement nommée chrysanthème des Indes, est la plante qu'on cultive le plus à la Chine, pour la décoration des jardins, parce que ses différentes variétés offrent toutes les nuances de couleur, excepté le bleu. Elle n'était pas encore connue en Europe, lorsqu'en ${ }_{1789}$, M. Blancard, négociant de Mar. 
244 DESCRIPTION DU MUSEUM.

peuvent nous arriver par la voie du commerce: mais que les petites plantes qui ne sont point encore cultivées, ne s'introduiraient jamais chez nous, si les graines n'en étaient recueillies par des naturalistes qui les envoient à l'école de botanique.

Ainsi nous n'aurions pas le réséda envoyé d'Égypte par Granger en I 736 , l'héliotrope du Pérou envoyé par Joseph de Jussieu en I 740 , le siléné bipartita apporté de Barbarie par M. Desfontaines en 1786 , si les graines de ces plantes n'eussent été adressées au professeur de botanique pour les semer à l'école, où on les a d'abord remarquées et d'où elles se sont répandues dans nos jardins.

Nous pouvons en dire autant de la jolie sabline de Mahon, arenaria balearica; de la lopezia du seille l'ayant apportée de la Chine, en donna des boutures à M. l'abbé Ramatuel. Celui-ci l'enroya au Jardin du Roi en ${ }^{179} 1$ : elle fut d'abord placée dans l'orangerie, ensuite en pleine terre, et comme elle se multiplie très-facilement, elle fut bientot répandue dans les jardins : elle a passé en Angleterre en $379^{5}$. M. Ramatuel en publia la description en $179^{2}$ dans le Journal d'histoire naturelle, tom. 2; et il prouva qu'elle différait du chrysanthemum indicum. C'est une acquisition précieuse, parce qu'elle fleurit au milieu de l'automne lorsque les autres fleurs disparaissent, et qu'elle résiste même aux premières gelées. Il n'est aucune plante d'ornement qui s'accommode aussi bien des différens climats; elle réussit dans la Belgique et à l'ille de France.

M. l'abbé Ramatuel, à qui le Jardin du Roi doit l'introduction de cette plante, est auteur d'un excellent mémoire sur les bourgeons, dont M. Desfontaines a donné un extrait dans le Journal de physique, tome 42, page 62. Il est mort à Paris en $179^{3}$. 


$$
\text { ÉCOLE DE BOTANIQUE. } 24.5
$$

Mexique que nous avons reçue d'Espagne il y a quelques années; du tussilage odorant, trouvé dans les Pyrénées et décrit par M. Villars; du cacalia sagitata que nous avons depuis peu reçu de Java, et de cent autres plantes qui se trouvent actuellement sur le quaiauxFleurs, parce que nous en avons distribué les graines.

En replantant l'école, M. Desfontaines a eu soin d'espacer les plantes de manière qu'on pût y placer les espèces nouvelles qu'on présumait pouvoir bientôt arriver. Déjà plusieurs de ces intervalles sont remplis par les richesses que nous avons reçues, surtout de la Nouvelle-Hollande; et peut-être ne se passera-t-il pas dix ans sans qu'on soit obligé d'agrandir encore un local qui a déjà été augmenté d'un quart depuis I 788 . 
\$ III. ÉCOLE DES ARBRES FRUTTIERS.

L'ÉCOLE des arbres fruitiers contient aujourd'hui plus de onze cents espèces ou variétés d'arbres et arbrisseaux, qui sont disposés dans un ordre méthodique, et placés les uns à côté des autres d'après l'analogie qu'ils ont entre eux.

L'enclos qu'elle occupe est un carré long qui a 40 toises de l'est à l'ouest, entre l'école de botanique et celle des plantes d'usage, et 27 toises et demie du nord au sud, entre l'allée des marroniers et celle des tilleuls. Elle est coupée dans sa longueur par une allée principale, et divisée transversalement en soixante planches de trois pieds et demi de large, séparées par des sentiers de trois pieds. Chaque planche est bordée d'une variété particulière de fraisiers. La distance entre les arbres est de trois à neuf pieds, selon leur grandeur.

Les premières planches sont occupées par les arbres ou arbrisseaux dont le fruit est une baie, comme les groseilliers, les framboisiers, les vignes, les mûriers. Dans la seconde division viennent les arbres à fruit à noyau, comme les cerisiers, les pruniers, les abricotiers. Dans la troisième, les fruits à osselets, comme les néfliers, les azero- 
ECOLE DES ARBRES FRUITIERS. 2 f liers, les plaqueminiers. Dans la quatrième, les fruits à pepins, tels que les pommiers, les согmiers ou sorbiers, et les fruits juteux, tels que la figue. Dans la cinquième, les fruits dont on mange seulement l'amande qui est renfermée dans une coque osseuse ou cartilagineuse : cette cinquième classe peut se subdiviser en deux, celle où la coque qui contient l'amande est nue, comme dans les pins et les noisetiers, et celle où elle est enveloppée d'un brou, comme dans les noyers et les châtaigniers.

Au bas de la plantation, on roit quelques pêchers en espalier, et d'autres arbres qui ne sont point taillés en quenouille, comme ceux qui précèdent.

Cette école a été plantée en I 792, lorsque M. Roland était ministre de l'intérieur, et M. de SaintPierre intendant du Jardin. Pour la former, on obtint du ministre la permission de faire prendre deux individus de chaque espèce dans la fameuse pépinière des Chartreux, el dans celle de Vitry, dont les propriétaires avaient fourni à 1)uhamel les sujets qu'il a décrits dans son Traité des arbres fruitiers, et les noms qu'ils avaient adoptés pour les désigner. D'où il suit que l'école du Jardin du Roi offre toujours le type des espèces décrites par Duhamel, et peut en certifier la nomenclature.

Les arbres réunis dans cette plantation sont, 
pour la plupart, greffés rez terre et taillés en quenouille. On a choisi cette taille, non point comme celle qui est en général la plus avantageuse, mais parce qu'elle économise le terrain, parce qu'elle met à portée de l'oeil de l'observateur les bourgeons, les feuilles et les fruits de l'arbre, et parce qu'elle fait pousser des scions plus longs et plus vigoureux; ce qui donne le moyen d'avoir un plus grand nombre de greffes. On a eu pour but de multiplier les espèces, de les répandre et d'en faciliter l'étude; et non d'indiquer comment il faut conduire les arbres pour leur faire produire beaucoup de fruits, et pour les faire durer le plus long-temps possible.

On a envoyé une collection complète des arbres cultivés dans cette école, à Gand, à Strasbourg, à Vienne en Autriche, etc., où l'on a établi des écoles d'arbres fruitiers sur le même modèle, dans le même ordre et avec les mêmes noms; ce qui est non-seulement utile pour propager les espèces, mais pour en rendre la nomenclature uniforme dans tous les pays : car les mêmes fruits ont été jusqu'ici désignés par des noms différens dans les diverses contrées et par les divers cultivateurs ; et malgré les descriptions qu'on trouve dans les livres, il est souvent difficile de décider si l'espèce qu'on possède est ou n'est pas celle qui est ailleurs désignée sous le même norm. 
ÉCOLE DES ARBRES FRUITTER. $2 / 9$

Il y. a peu d'espèces remarquables qui ne se trouvent aujourd'hui dans l'école du Muséum: Lors de la première plantation on n'avait que celles dont Duhamel a donné l'histoire; on s'en cst depuis procuré beaucoup d'autres qui, n'existant que dans les pays étrangers, ou dans certains cantons fort éloignés de la capitale, étaient presque entièrement ignorées. Le nombre en est à peu près double de ce qu'il était d'abord, et chaque année elle s'enrichit de quelque acquisition nouvelle. Ce qu'elle possède est bientôt communiqué aux cultivateurs, et quoiqu'elle n'existe que depuis vingt-neuf ans, elle a déjà répandu dans nos jardins et nos vergers plusieurs espèces qui étaient auparavant fort rares et à peine connues.

Le coup d'œil que présente cette plantation est extrêmement curieux pour ceux qui ne font que la parcourir, et très-instructif pour ceux qui veulent étudier. On y voit toutes les variétés rapprochées les unes des autres selon leurs affinités, ce qui donne le moyen de les comparer. Les fruits des différentes saisons s'y succèdent depuis le mois de mai jusqu'au mois de novembre, ils y ont disparu dans certaines variétés tandis qu'ils ne sont pas encore mûrs dans d'autres. On peut en hiver y étudier les caractères qui font distinguer les variétés par la couleur du bois et la forme des boutons; connaissance très-nécessaire aux culti- 
250 DESCRIPTION DU MUSECM.

vateurs, puisque c'est après la chute des feuilles que se font les plantations.

On n'a point encore imprimé le catalogue de cette collection, parce qu'on attend d'avoir cer tifié la nomenclature en usage dans tous les pays. Mais un catalogue manuscrit, correspondant à des numéros placés au pied des arbres, est entre les mains du jardinier de l'école, et ce jardinier nomme à tous ceux qui le demandent les arbres qu'ils ne connaissent pas.

Les personnes qui veulent avoir des greffes, peuvent s'adresser à M. Thouin, professeur de culture, en lui désignant les espèces qu'elles désirent, soit par un nom connu, soit par le numéro qu'elles portent à l'école.

Parmi les espèces rares ou nouvellement introduites, on remarque aujourd'hui dans cette plantation un très-bel individu du diospyros kaki ou plaqueminier du Japon, dont les fruits, de la grosseur d'une pormme d'api, sont fort recherchés dans ce pays; un très-beau bibacier ou néflier du Japon (mespilis japonica), qui passe l'hiver en pleine terre et donne de bons fruits; le pin de Monteray, envoyé de la Californie lors du voyage de La Peyrouse, et dont les amandes sont encore meilleures que celles du pin à pignons; le cognassier du Japon et le poirier du mont Sinaï, espèces curieuses; de beaux noisetiers du Levant, etc. 
ECOLE DES ARBRES FRUITERS. 25 ?

On sent bien que l'école des arbres fruitiers ne peut être indistinctement ouverte au public. Fille est séparée du reste du jardin par un treillage de huit pieds d'élévation : mais on n'en refuse l'entréc à aucune des personnes qui désirent la visiter pour leur instruction. 
§ IV. ÉCOLE DES PLANTES D'USAGE DANS L'ÉCONOMIE DOMESTIQUE, ET DANS LES ARTS.

CetTe école qui fait suite à celle des arbres fruitiers, est limitée au nord par l'allée des marroniers, au midi par celle des tilleuls, au levant par celle des catalpas, au couchant par celle des platanes. Elle est entourée d'un treillage en bois de trois pieds de hauteur. Sa forme est un carré de 36 toises 4 pieds de long, sur 3o toises et demie de large. Dans l'intérieur sont deux grandes allées, l'une qui la partage dans sa longueur, l'autre qui en fait le tour. Les deux carrés longs traversés par l'allée du milieu, sont divisés chacun en vingttrois planches de 6 pieds de large, qui sont ellesmêmes divisées en petits compartimens d'une toise. Ces carrés au nombre de cinq cent cinquante-deux, sont destinés à recevoir autant d'espèces ou de variétés de plantes économiques.

Ces plantes ne sont pointrangées selon une méthode de botanique, mais par ordre de propriétés, pour que les cultivateurs puissent les étudier d'après le but qu'ils se proposent. Elles sont distribuées en trois groupes principaux. 
ÉCOLE DES PLANTES ÉCONOMIQUeS. 253

La première division comprend les plantes utiles à la nourriture de l'homme.

La seconde, celles qui sont propres à nourrir les bestiaux.

La troisième, celles qui sont employées dans les arts.

Une étiquette particulière au commencement de chaque bande, indique l'usage des végétaux qui y sont cultivés.

Chacun de ces groupes comprend plusieurs sections. Ainsi le premier se compose des céréales, des semences farineuses, des plantes potagères, des semences oléifères; et chacune de ces sections est encore divisée d'après les parties de la plante qui sont les plus recherchées. Ainsi dans les plantes potagères on distingue celles dont on mange les racines, comme la patate, le topinambour, la scorsonère ; celles dont on fait cuire les feuilles, comme les choux, les épinards et l'oseille; celles dont on mange les calices ou les fleurs, comme l'artichaut, le chou-fleur, la capucine, ou les fruits, comme les courges et les melons; celles dont les semences aromatiques sont employées pour assaisonnement, comme l'anis, la corriandre, etc.; enfin celles qu'on mange en salade, comme la laitue, la chicorée, la mâche. Dans les plantes propres aux arts on distingue. des textiles, comme le lin et le chanvre, et les 
tinctoriales, comme la garance et le pastel : dans les plantes pour la nourriture des bestiaux; les fourrages qui appartiennent aux graminées, ceux qui appartiennent aux légumineuses, et les herbes de pâturage.

Enfin une dernière section est réservée aux plantes qui ont quelque usage particulier, comme le tabac; le houblon, la cardère, etc.

Dans une école de botanique il faut que les plantes soient isolées pour qu'on puisse mieux étudier leurs caractères distinctifs, mais dans le but qu'on s'est proposé ici il faut qu'elles soient disposées par petits massifs, comme elles doivent l'être dans les champs ou les jardins : il serait à désirer que ces massifs eussent une étendue quadruple, ou du moins double de celle qu'on leur a donnée, mais le local ne l'a pas permis.

La conduite de cette école exige des précautions particulières, on est obligé d'alterner les cultures pour ne pas mettre plusieurs années de suite les mêmes plantes dans le même terrain. Ainsi l'on commence par un des deux bouts de l'école, et deux ans après par l'autrè bout, ce qui conserve toujours le même ordre en changeant les espèces de place. On est obligé aussi de se procurer fréquemment de nouvelles graines, parce que les variétés dégénèrent lorsqu'elles sont pendant quelques années cultivées dans lc même local, 
ÉCOLE DES PLANTES ÉCONOMIQUES. 255 et que les diverses variétés étant placées près les unes des autres, il peut arriver qu'elles se fćcondent mutuellement, ce qui produit des espèces mixtes, et fait perdre le type primitif.

Les semis dans cette école se font à trois époques selon la nature des plantes; à la fin de l'automne, au commencement du printemps et au commencement de l'été. A mesure qu'elles mûrissent on en recueille les graines pour les distribuer aux cultivateurs.

On en fait annuellement vingt mille sachets dont l'étiquette indique non-seulement le non de la plante, mais encore l'époque à laquelle il faut la semer, le terrain qui lui convient et son principal usage.

I'école de culture ne présente point aux yeux un aspect aussi agréable qu'un parterre de fleurs ; mais elle a un caractère singulier par les teintes variées des divers carrés, et par la différence de hauteur des plantes qu'ils contiennent. Ces compartimens offrent en petit.l'image d'une vaste campagne, ou d'une ferme expérimentale, où l'on aurait destiné un champ particulier à chacun des végétaux herbacés qui sont utiles à l'homme et qui peuvent croître dans nos climats. L'agriculteur et le jardinier y apprennent à connaître les plantes qui sont cultivées de préférence dans divers cantons dont elles font la richesse; celui 
256 DESCRIPTION DU MUSÉUM.

Iui s'occupe d'un objet particulier, y trouve rapprochées sur des planches voisines, toutes les plantes tinctoriales, toutes les plantes textiles, toutes celles enfin qui se suppléent pour une certaine destination.

Parmi les plantes sorties de cette école, on peut citer le lin de la Nouvelle-Zélande, les patates de Pensylvanie, qui sont aujourd'hui répandues dans le midi de la France, le cresson de Para (I), le tetragonia expansa (2), le claytonia cubensis (3), etc.

Cette école est la seule de ce genre qui existe en Europe. Lorsqu'on en eut arrêté le plan, peu de temps après la nouvelle organisation du Muséum, on fut obligé d'exaucer le terrain de huit pieds pour le mettre au niveau de l'école des arbres fruitiers. On remplit le fond de décombres, et l'on couvrit le sol d'un pied de bonne terre.

On sent bien que la culture de cette école exige des soins et des procédés variés selon la diversité des plantes; nous n'entrerons à ce sujet dans aucun détail. Nous nous bornerons à renvoyer pour en connaitre la conduite, l'organisation et l'utilité, au mémoire que M. Thouin a inséré dans les Annales du Muséum, tome 2, page 142.

(1) Spitanthus oleracea. Lorsqu'on s'en frotte les gencives, il excite la salivation et dissipe souvent les douleurs de dents.

(2) Bon légume, apporté de la Nouvelle-Zélande par sir J. Banks.

(3) Plante potagère que nous devons à M. Bonpland. 


\section{$\S$ V. ÉCOLE DE CULTURE.}

CetTe école; située vers le bas du jardin; et séparée de celle des plantes économiques parl'allée des catalpas de Virginie, a été créée en I 806 , et c'est la première et peut-être la seule qui ait été formée en Europe. Elle est destinée à mettre sous les yeux ce qui est relatif à la culture des végétaux, à offrir des modèles des diverses pratiques employées pour leur éducation et leur multiplication, et à confirmer ou rectifier par l'expérience les notions que nous avons sur la physique végétale. Quoique son étendue ne soit pas aussi considérable que l'exigerait un pareil établissement, et que la surface en soit horizontale, aucune partie du jardin ne présente un aspect aussi curieux et aussi varié.

Sa forme est un carré long, de $35^{\circ}$ toises du levant au couchant, et 3 o du nord au sud. Elle est entourée d'un treillage de 4 pieds d'élévation, encadrće intérieurement par une allée de 4 pieds de large, et traversée dans sa longueur par une allée de 6 pieds. L'espace qu'elle occupe est divisé en quarante-huit planches, qui ont de 3 pieds à 6 pieds de largeur selon leur destination. 
Pour que cette école fût distribuée d'après ur plan méthodique et régulier, on a divisé en quatre classes les objets sur lesquels on voulait donner des exemples. La première comprend ce qui est relatif au moyen de faire naître les végétaux; la seconde, ce qui tient à leur conservation; la troisième, les moyens de les multiplier ; la quatrième, les usages auxquels on emploie, dans les campagnes et les jardins, la réunion de plusieurs végétaux vivans.

On a été obligé de placer les exemples de la troisième classe avant ceux de la seconde, pour que les boutures et les marcottes, qui craignent les rayons du soleil, se trouvassent ombragées par l'allée des catalpas, et par les arbres des plantations.

Comme il n'y a qu'un seul moyen de faire naître les végétaux, tandis qu'il en est plusieurs de les conserver et de les multiplier, l'ensemble des classes que nous venons d'indiquer a été partagé en dix sections, dont chacune occupe plus ou moins de planches, selon le nombre des exemples qu'elle exige. Voici l'ordre dans lequel ces divisions se suivent :

I $^{\circ}$ Les semis, en pleine terre, sur couche, sous châssis, en pot, dans l'eau, sur d'autres végétaux, et les pratiques qui facilitent la levéc des graines, et en assurent le développement. 
$2^{\circ}$ Les boutures et les divers procédés qui en facilitent la reprise, lorsque les végétaux ne sont pas disposés à pousser des racines de leurs branches.

$3^{\circ}$ Les marcottes et les soins qu'elles exigent.

$4^{\circ}$ Les greffes de différentes sortes, et les essais, presque toujours infructueux, qu'on a faits pour la réussite des greffes hétéroclites ( $\mathrm{r}$ ).

$5^{\circ}$ Les plantations et les divers moyens d'en assurer le succès; moyens variés selon la nature des arbres, le pays duquel ils sont originaires, l'époque à laquelle ils entrent en séve.

$6^{\circ}$ La manière de tailler les arbres, soit pour les faire durer long-temps, soit pour leur donner la forme qu'on désire, soit pour leur faire porter des fruits plus gros, plus savoureux, ou en plus grande quantité.

$7^{\circ} \mathrm{La}$ taille et la conduite de la vigne, soit en plants isolés, soit en berceau, soit ẻn espalier.

$8^{n}$ Les haies, divisées en haies de défense, haies greffées, haies simples et doubles, haies de fourrage.

(1) Ces expériences faites avec beaucoup de soin, et répétées de toutes les manières, ont du moins servi à prouver que les arbres qui n'ont entre euz aucune analogie, ne peuvent être greffés les uns sur les autres, et que tout ce qu'on a écrit, depuis Columelle jusqu'à nos jours, sur les moyens de se procurer par la grefle des espèces bybrides et des fruits extraordinaires, est dénué de fondement. 
$9^{\circ}$ Les palissades, divisées en trois espèces : les palissades de príntemps, celles d'été, celles d'hiver.

$10^{\circ}$ Les fossés; manière de les construire, d'en revêtir et d'en décorer les talus, et de les garnir de haies pour leur défense.

Le choix des modèles dont nous venons d'indiquer la série, présente les divers procédés applicables à la culture des végétaux selon le but qu'on se propose, et les expériences les plus propres à nous éclairer sur les phénomènes de la régétation. On ne sentira cependant toute l'utilité de ces différens modèles qu'autant qu'on les examinera avec attention, qu'on les comparera dans tous les détails, et qu'on pourra se faire expliquer comment plusieurs d'entre eux sont parvenus au point où on les voit. Mais la distribution de ces mêmes modèles et la manière dont ils sont groupés offrent dans l'ensemble un aspect aussi agréable par les contrastes, qu'il est instructif par les rapprochemens.

Ainsi dans les planches destinées à la taille des arbres, on voit à côté l'une de l'autre les tailles en quenouille, en boúle, en buisson, en vase, en éventail, en espalier, et l'arcure (r) par laquelle on

(1) On a été obligé de renoncer à ce procédé : le premier résultat qu'on en obtient est une fécondité surprenante; mais dès la seconde année les branches arquées s’épuisent, et l'arbre lui-même ne tarde pas à dépérir. 
ÉCOLE DE CULTURE.

261

fait produire plus de fruits aux arbres, en en courbant les branches en demi-cercle, et détournant ainsi le cours de la séve. On y voit aussila manière de hâter la maturité des fruits, et de les empêcher de couler, en enlevant au printemps un anneau d'écorce sur la branche qui doit les porter, ou bien en interrompant le cours de la séve par des liyatures.

Dans les planches où sont les modèles de greffes, on voit plusieurs arbres unis par le haut de leur tronc, de manière qu'une seule tête est nourrie par quatre ou cinq pieds qui sont à l'entour. D'autres greffes présentent les branches de plusieurs arbres courbées en cerceau et réunies les unes aux autres par leur sommet, de manière à former un berceau couvert par des guirlandes de fleurs et de fruits. Quelques arbres portent sur le même pied autant d'espèces de fruits qu'il y a de branches principales : de jeunes sujets venus de graines semées dans l'année et n'ayant encore que quinze à dix-huit pouces de hauteur, ont reçu la greffe d'un arbre adulte et en nourrissent et font mûrir les fruits. On y voit enfin des greffes par lesquelles on est parvenu à donner au tronc et aux branches des arbres une forme singulière, quiles rend propres à fournir descourbes naturelles très-solides pour certains ouvrages de charpente. 
Les exemples de palissades, de haies et de fossés revêtus de diverses plantes sur les bords, offrent également un coup d'œil très-curieux.

Quoique la plantation de cette école ait été commencée il y a seize ans, c'est seulement depuis un petit nombre d'années qu'elle attire les regards, et qu'elle paraît répondre au but qu'on s'était proposé. Plusieurs des parties qui la composent ne présentaient d'abord rien d'intéressant, parce que ni les berceaux, ni les arbres greffés, ni ceux auxquels on avait voulu donner par la taille une forme particulière n'avaient encore acquis leur développement : elle est aujourd'hui à peu près ce qu'elle doit être et l'on ne saurait imaginer dans un aussi petit espace, un jardin dont l'effet soit plus pittoresque.

On y voit près de l'entrée, des berceaux ou cabinets de verdure formés par la greffe de deux lignes d'arbrisseaux revêtus de feuillage au sortir de terre, et dont les branches réunies en voûte sont tellement touffues, que le moindre rayon de soleil ne peut y pénétrer : plus loin, des arbres en pyramide très-élevés, et d'autres en vase dont les branches ont dix-huit pieds de circonférence : vers le bas, des palissades de genévrier du vert le plus brillant et qui ont vingt pieds de hauteur : enfin des fossés bordés d'une haie vive, et dont les talus, garnis de petites plantes couvertes de 
Acurs, présentent à l'œil sur un plan incliné une mosai̊que extrêmement agréable.

M. 'Thouin, professeur de culture, qui a formé cette école et qui en a seul la direction, y fait une partie de ses leçons. Il peut ainsi montrer aux élèves les exemples de tous les procédés dont il leur a parlé, et leur en faire remarquer les résultats sur la nature vivante.

Ceux qui ne peuvent suivre les cours de M. Thouin, et qui veulent visiter l'école de culture pour leur instruction, et non comme un objet de curiosité, doivent lire les mémoires que ce professeur a insérés dans les Annales du Muséum, et dans lesquels il a expliqué tous les procédés des semis, des boutures, des marcottes et des yreffes dont les exemples sont à l'école. Ils y trouveront le résultat d'un grand nombre d'expériences dont quelques-unes ne sont pas d'une utilité immédiate pour augmenter les produits de la végétation, mais qui toutes sont propres à résoudre des questions de physique végétale, et donnent sur cette science des notions fécondes en conséquences, non-seulement pour la théoric mais encore pour la pratique (I).

(i) M. Thouin a confié le soin de cette école et de celle des arbres fruitiers, à M. Dalbret, jardinier qu'il a lui-même instruit dans la taille des arbres et dans les pratiques de culture, et qui est en état de donuer aux personnes qui lui sont adressées par les professeurs, les explications qu'elles désirent. 


\section{\$ VI. JARDIN DES SEMIS.}

$C_{E}$ jardin qui seul entretient, accroît et renouvelle nos richesses en botanique, n'existe que depuis r786. Le terrain qu'il occupe avait été acquis l'année précédente par Buffon, qui en confia l'ordonnance à M. Thouin. Ce professeur en a publié, dans les tomes 4 et 6 des Annales du Muséum, une description détaillée. Nous y renvoyons ceux de nos lecteurs qui voudront connaître l'usage de toutes les parties dont il est composé, et s'instruire en même temps des divers procédés employés pour semer avec méthode, pour faire lever, et pour conduire jusqu'au moment de la transplantation les végétaux de tous les climats. Nous croyons devoir nous borner ici à faire remarquer l'ordre dans lequel tout y est distribué, et le but qu'on s'est proposé dans cette distribution.

Le jardin des semis a 885 toises carrées (ou 3362 mètres carrés) de surface. Il est enfoncé d'environ ro pieds au-dessous du sol de l'ancien jardin, et de niveau avec le jardin de naturalisation qui, formé en même temps et placé à son 
extrémité orientale, n’en est séparé que par un mur de clôture. Il est borné au nord par la serre tempérée, au couchant par la colline plantée d'arbres verts, au sud par l'allée des marroniers. Cette situation le met parfaitement à l'abri des vents du nord et de l'ouest.

En suivant au dehors le mur qui soutient les terres au-dessous desquelles il est situé, on en voit parfaitement la distribution, et l'on reconnaît même la plupart des plantes qui y sont en fleur.

La porte d'entrée est au hout de la terrasse de 200 pieds de long qui occupe le devant de la serre tempérée. Pendant la belle saison cette terrasse garnie des arbres et arbrisseaux qui ont passé l'hiver dans la serre, offre un coup d'oil superbe, et dont on jouit lorsqu'on se promène dans l'allée des marroniers.

Près de la porte et à droite est un escalier par lequel on descend dans le jardin. Au mur qui soutient la terrasse est adossée dans toute sa longueur une ligne de huit châssis dans lesquels sont encaissées des couches chaudes, où l'on place les semis des graines venues des pays chauds. Cette ligne est interrompue dans son milieu par une petite serre. Les encaissemens de chaque côté de la serre sont au nombre de quatre : ils sont en fer et couverts par des châssis vitrés, inclinés et mobiles sur des crémaillères, de sorte qu'on les 
ouvre à volonté pour donner de l'air aux plantes. Au-dessous de ces huit encaissemens, et sur la même ligne, il y en a huit autres en maçonnerie, où l'on place sur couche les plantes bulbeuses ou tubéreuses du cap de Bonne-Espérance et des climats analogues : ces derniers ne sont couverts de châssis que pendant l'hiver. A l'extrémité de la ligne des couches est un escalier qui remonte sur la terrasse, et qui correspond à celui par lequel on est entré.

Le long du mur qui soutient les couches garnies de liliacées, d'iridées et de narcisses, est une petite allée, à droite de laquelle sont deux lignes de couches simples destinées aux semis des plantes de la zone tempérée. Elles ont 6 pieds de largeur et 25 toises de longueur. Leur hauteur lorsqu'elles sont nouvellement construites est de 2 pieds 6 pouces , qui se réduisent à Io pouces lorsqu'elles se sont affaissées. Elles peuvent contenir ensemble cinq mille pots qui y sont arrangés dans l'ordre des numéros du catalogue des semis. Cette double ligne de couches simples, dont une partie est quelquefois couverte de châssis vitrés, est coupée dans son milieu par l'allée transversale qui est vis-à-vis de la petite serre.

Au-dessous de ces couches et dans une direction contraire, c'est-à-dire du nord au sud, sont trente-sept planches de longueur inćgale, et larges 
de 5 pieds. Les vingt-neuf premières sont destinées aux semis des plantes annuelles dont la végétation s'accomplit en quelques mois, et qu'on ne sème que lorsque la terre est déjà imprégnée de chaleur. On s'en sert aussi pour repiquer quelques plantes annuelles qui ont levé dans les pots et dont on veut recueillir les graines.

Les huit planches suivantes sont des couches froides pour la transplantation, les séparations et les repiquages des jeunes plants qu'on a nouvellement obtenus en semant les graines des arbustes et des plantes vivaces qui sont originaires des zones froides et tempérées : les pots qui contiennent ces jeunes plantes y sont enfoncés dans le terreau, et l'on prend les précautions nécessaires pour les abriter du soleil dans les premiers jours. Lorsque la reprise est bien assurée, on transporte ces plantes soil dans la serre tempérée soit dans les parterres.

Après toutes ces planches viennent deux banquettes à l'exposition du couchant : ce sont des couches sourdes destinées à recevoir des pots de semis dont les graines n'ont pas levé dans l'année, pour qu'elles puissent se développer les années suivantes.

A l'est de ces banquettes est une plantation de thuyas fort élevés; entre ces thuyas et le mur de clôtur'e est un espace où l'on a construit une 
petite cabane : c'est là que se place le jardinier en chef avec ses aides pour faire les semis. A droite et à gauche on dépose dans des abris, diverses choses nécessaires à la culture, comme outils, paillassons, cloches, etc.

En revenant de l'est à l'ouest, dans un sentier qui est à dix pieds du mur de terrasse, on suit une ligne de couches sourdes sur lesquelles on place les pots où sont semées la plupart des plantes des pays froids et quelques-unes des pays tempérés, dont les graines sont très-fines. Ces couches sont garanties du soleil du midi par le feuillage des marroniers de l'allée. Aux deux extrémités de ces couches sont quelques vases remplis d'eau pour les semis des plantes aquatiques.

Le long du mur est une plate-bande gui reçoit pendant quelques heures les rayons du soleil à son lever et à son coucher, et qui en est absolument abritée dans le milieu du jour. C'est là qu'on sème et qu'on élève pendant leur jeunesse les arbustes et les grandes plantes vivaces des régions les plus froides.

Cette plate-bande est partagée dans son milieu par un passage souterrain et voûté qui conduit à l'école de botanique; et les deux parties de la banquette à droite et à gauche du passage sont composées de terres différentes qu'on a préparées pour les plantes auxquelles elles sont destinées. 
()n voit ici de très-jolies fougères du nord, des veratrum, des ombellifères, des daphnés, des gentianes et des géranium des Alpes.

Lorsqu'on est arrivé à l'extrémité de cette plate-bande, on trouve le long du mur de la terrasse qui est à l'ouest un amphithéâtre composé de cinq gradins, chacun de dix pouces de haut et d'un pied de large, et partagés dans leur longueur en compartimens de 8 pieds ı pouces. Cet amphithéâtre est exposé au nord-est, et ne reçoit le soleil que jusqu'à dix heures du matin. Avant de le construire on a creusé dans l'emplacement qu'il devait occuper un fossé qu'on a enduit d'un mortier impénétrable à l'eau; on a rempli ce fossé de bonne terre; on a placé au-dessus les gradins, qui sont en planches de chêne soutenues par des poteaux ; et chaque gradin a été couvert de terreau de bruyère. Cette construction conserve l'humidité en empêchant que l'eau des arrosemens ne se perde dans le sol dont le fond est sablonneux.

C'est sur ces gradins qu'on sème et qu'on élève les plantes des régions polaires et celles qui croissent sur les montagnes au pied des neiges. Elles y réussissent fort bien : presque toutes sont de petite taille, et se couvrent au printemps de trèsjolies fleurs. On en porte toujours un pied à l'école, mais elles n'y durent pas long-temps, tandis 
que sur les gradins elles conservent leur fraîcehur jusqu'à l'époque de la maturité des graines. On peut y voir aussi élégantes que dans leur sol natal le moehringia muscosa, la violette à fleurs jaunes (viola biflora), des androsacés, des prime vères et des saxifrages des Pyrénées, la soldane lle des Alpes, l'absinthe des glaciers, les épilobes, les siléné, et les renoncules qui croissent naturellement près des neiges, des saules nains, et quelques autres arbustes du Groënland et du Haut-Canada.

A l'extrémité de ces gradins on trouve l'escalier par lequel on remonte sur la terrasse.

Nous avons dit que la ligne de châssis et de couches appuyée contre le mur de la terrasse couverte d'arbrisseaux, était coupée dans son milieu par une petite serre vitrée. Cette serre n'y est placée que depuis trois ans. Elle appartenait à M. Delaunay, sous-bibliothécaire du Jardin, amateur fort instruit, et auteur d'un ouvrage très-répandu intitulé l'Almanach du bon Jardinier ; elle lui servait à cultiver quelques plantes agréables. A sa mort elle a été acquise par le Muséum. On s'en sert pour faire des élèves des plantes de la Nouvelle-Hollande et du Cap, qu'on n'ose pas, dans leur première jeunesse, placer dans la serre tempérée où elles n'auraient pas assez de chaleur;

Vis-à-vis de cette serre et au milieu de l'allée transversale qui partage le jardin du nord au sud 
est un bassin qui communique à un puits. Malheureusement l'eau de ce puits est tellement séléniteuse qu'elle encroûte les racines, et qu'on est obligé d'envoyer chercher de l'eau à la rivière pour les plantes un peu délicates. On cessera de s'en servir lorsqu'on aura fait arriver au Muséum de l'eau du canal de l'Ourcq. Entre le bassin et le puits sont plusieurs auges en pierre destinées à des plantes aquatiques.

La description succincte que nous venons de donner du jardin des semis suffit pour faire juger de la variété des travaux qu'il exige et de l'intérêt qu'il présente.

Nous n'avons pas besoin d'ajouter que chaque année on y découvre des plantes non encore décrites, et venues de graines envoyées sans nom par des voyageurs. On prend tous les soins possibles pour les conserver et les multiplier; on en transporte d'abord quelques individus dans le jardin de naturalisation, et sitôt qu'on en a obtenu des graines, on les distribue aux botanistes et aux cultivateurs.

La direction de ce jardin est exclusivement confiée à M. Jean Thouin, jardinier en chef, qui y donne ses soins pendant toute l'année, et particulièrement à l'époque des semis. Il ne s'en repose sur qui que ce soit pour cette opération délicate, et il a soin de visiter chaque jour les pols 
pour savoir si quelque graine a levé, et pour arracher les mauvaises herbes. C'est lui aussi qui préside à la préparation des diverses terres, et qui fait porter chaque plante à la place qui lui convient.

Lorsque M. Jean Thouin fait les semis, il met dans chaque pot en même temps que la graine, un numéro gravé sur une plaque de plomb : ce numéro renvoie à un catalogue où se trouve le nom de la plante lorsqu'elle est connue, et dans le cas où elle ne l'est pas, le pays d'où elle vient, le nom de celui qui l'a envoyée, et l'époque à laquelle on l'a reçue. On a soin d'ajouter sur le catalogue la date du jour où la graine a été semée. On sent que ces détails minutieux exigent beaucoup de soins, un goût particulier pour les plantes, et une grande habitude des diverses pratiques de culture.

Le jardin des semis est, comme nous avons dit, la pépinière où l'on prend ce qui doit peupler toutes les autres parties, où l'on multiplie et renouvelle nos richesses végétales. Mais il présente sous les rapports de la science un autre genre d'utilité qu'on ne saurait trouver ailleurs : c'est que c'est là, et là seulement, qu'on peut faire des observations méthodiques et suivies sur la germination des graines et comparer les plantes dans le premier période de leur développement. On 
sait que l'évolution de la radicule, de la plumule et des cotylédons, fournit les caractères les plus importans pour la classification des végétaux et pour la détermination des rapports naturels : ces caractères peuvent sans doute être aperçus par la dissection des graines, mais ils sont bien plus apparens lorsque ces graines germent et que toutes leurs parties se développent successivement. C'est alors qu'on peut voir comment la vie se distribue, et quels sont les premiers phénomènes que produit son action : phénomènes de la plus haute importance pour la physiologie végétale. Or tout cela ne peut être saisi que dans un lieu où les plantes de tous les climats et de toutes les familles sont semées les unes à côté des autres. Alors leur rapprochement facilite la comparaison.

Aussi est-ce dans ce jardin des semis que M. Mirbel a fait sur l'évolution des graines et sur leurs caractères les belles observations qu'il a consignées d'abord dans les Annales du Muséum, et qu'il a reproduites avec de nouveaux développemens dans ses Élémens de botanique et de physiologie végétale.

Cependant ce jardin n'est point ouvert au public, le jardinier en chef et les professeurs de culture et de botanique en ont seuls la clef. On sentira facilement que cette précaution est indis- 
pensable pour que l'ordre des pots et celui des numéros qui les accompagnent ne soient jamais dérangés; pour qu'une plante souvent unique, et qui ne fait que de naître, ne soit point touchée; pour qu'on ne donne jamais mal à propos de l'air à celles qui dans certains momens ont besoin d'une chaleur concentrée. Mais les personnes instruites qui veulent visiter cette partie de l'établissement, soit pour observer la première germination des graines, soit pour y voir des plantes qui y sont en fleur, soit pour examiner s'il n'y a pas levé quelque espèce nouvelle, obtiennent toujours la permission de M. Jean Thouin qui les fait accompagner par un jardinier, et qui souvent a la complaisance de les accompagner lui-même pour leur donner les explications qu'elles peuvent désirer. Ce qu'on peut remarquer ici comme dans toutes les autres parties de l'établissement et qui ne se trouve peut-être nulle autre part en Europe, c'est qu'on n'y cache rien, et qu'on se plait à communiquer sans réserve tout ce qui peut servir à l'instruction des hommes studieux et aux progrès des sciences. Mais pour atteindre ce but il faut agir avec prudence et prendre toutes les précautions possibles pour la conservation des objets qu'il serait difficile de remplacer si on venait à les perdre. 
§ VII. JARDIN DE NATURALISATION.

Nous arons dit qu'à l'est du jardin des semis il y en avait un autre sur le même niveau, et dans lequel on entrait par une porte placée au milieu du mur de clôture derrière l'allée des thuyas. Cet enclos est, comme le précédent, enfoncé de ro pieds au-dessous de l'allée des marroniers et des terrains de la. ménagerie; sa largeur est à l'entrée la même que celle du jardin des couches, mais il se rétrécit en allant vers l'est; sa longueur est de 22 toises.

La face qui se présente au levant et qui est abritée du nord et de l'ouest par les murs, et par la palissade des thuyas, est destinée à recevoir pendant l'été la plupart des arbres et arbustes de la Nouvelle-Hollande qui ont passé l'hiver dans la serre tempérée. Lorsque les métrosidéros, les mélaleuca, les leptospermum, les eucalyptus, les banksia, les embothrium sont en fleur, cette partie du jardin présente un coup d'œil très-intéressant et très-agréable.

Le long des murs qui l'entourent des trois autres côtés et qui ont 15 pieds d'élévation, on place di- 
vers arbres ou arbustes selon l'exposition qu'ils exigent. Ainsi, au midi, on voit des pistachiers, des jujubiers, des grenadiers, l'ephedra altissima (I) apporté de Barbarie par M. Desfontaines, un lagerstrome de Chine (2), un très-beau chêne aux glands doux venu d'Espagne, des câpriers, etc. : au nord, des arbrisseaux et des plantes vivaces des pays froids, des spirea de Sibérie, plusieurs orchidées, quelques fougères.

Ce jardin est coupé transversalement dans son milieu par deux allées de thuyas qui sont fort rapprochés, et sous lesquels on élève en pots les plantes qui croissent dans les forêts les plus épaisses, et qui ont besoin d'être cultivées à l'ombre.

Au devant de ces allées est un puits et un bassin dont l'eau sert aux arrosemens. Près de ce puits est un mủrier à papier qu'on a laissé à cette place, parce que c'est un rejeton de celui qui nous fut envoyé par sir Joseph Banks et qu'on a transporté dans la ménagerie.

Le reste du jardin est divisé en plates-bandes destinées à la culture des plantes vivaces de pleine

(1) Arbrisseau dépourvu de feuilles, mais dont les rameaux grêles, pendans, et toujours verts, forment des touffes très-épaisses; il monte sur les autres arbres, et les couvre de sa verdure.

(2) Lagerstroemia indica, Lin. Arbrisseau cultivé à la Chine, dans les Moluques et dans les Indes orientales, à cause de la beauté de ses fleurs. 
JARDIN DE NATURALSATION. 277

terre les plus intéressantes et les plus rares, à celles qui ont été nouvellement introduites, et surtout à celles qui ne sont pas encore connues, qu'on cultive dans un lieu séparé pour les observer à toutes les époques de leur développement, et pour en recueillir les graines. Lorsque ces plantes sont bien déterminées on les transporte dans les parterres.

L'aspect de ce jardin vu de l'allée des marroniers ou de la terrasse qui le borne du côté de la ménagerie, est extrêmement pittoresque par la beauté et la variété des plantes étrangères qu'il renferme, et qui toutes élevées dans un bon terrain, et débarrassées par le sarclage du voisinage des herbes qui s'emparaient d'une partie de leur nourriture, développent en liberté le luxe de leur végétation et l'éclat de leurs fleurs.

Les botanistes qui voudraient examiner de plus près quelques-unes des plantes qui s'y trouvent, doivent s'adresser à M. Jean Thouin. 
\$ VIIT. SERRE TEMPÉRÉE.

LA grande serre tempérée construite sur les dessins de M. Molinos, architecte du ministère de l'intérieur, a été commencée en $179^{5}$ et terminée en 1800: sa longueur est de 200 pieds, sa largeur de 24 pieds, sa hauteur jusqu'au sommet de la voûte de 27 pieds. Les croisées au nombre de dix-sept ont 9 pieds et demi de large et près de 12 pieds de haut, sans y comprendre la partie cintrée qui ne s'ouvre pas, et qui a plus de 3 pieds. Elles posent sur une banquette en pierre de taille de r 9 pouces, et sont séparées l'une de l'autre par une colonne. La porte d'entrée qui est à l'ouest a Io pieds de largeur et 24 pieds d'élévation, pour qu'on puisse aisément faire entrer et sortir les arbres; une porte latérale sert pour le service journalier.

Sur le mur du fond, vis-à-vis la sixième et la douzième croisée sont deux grands poêles avec des tuyaux de chaleur; mais on n'y fait du feu que lorsque le thermomètre descend au dehors à quatre degrés au-dessous de zéro. Comme la façade estau midi, que la plus grande partie de sa surface est occupée par des vitres, et que les croisées fer- 


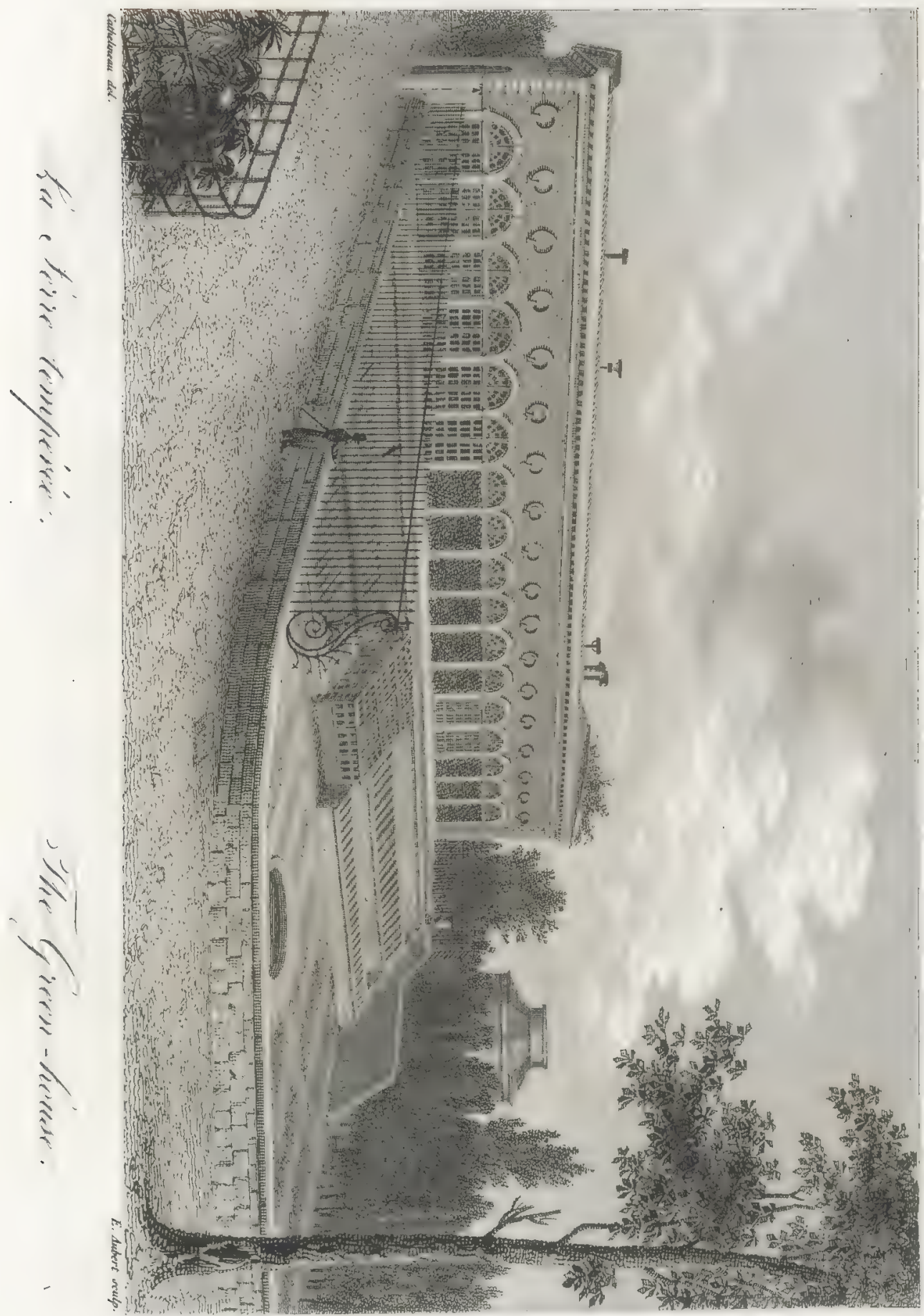




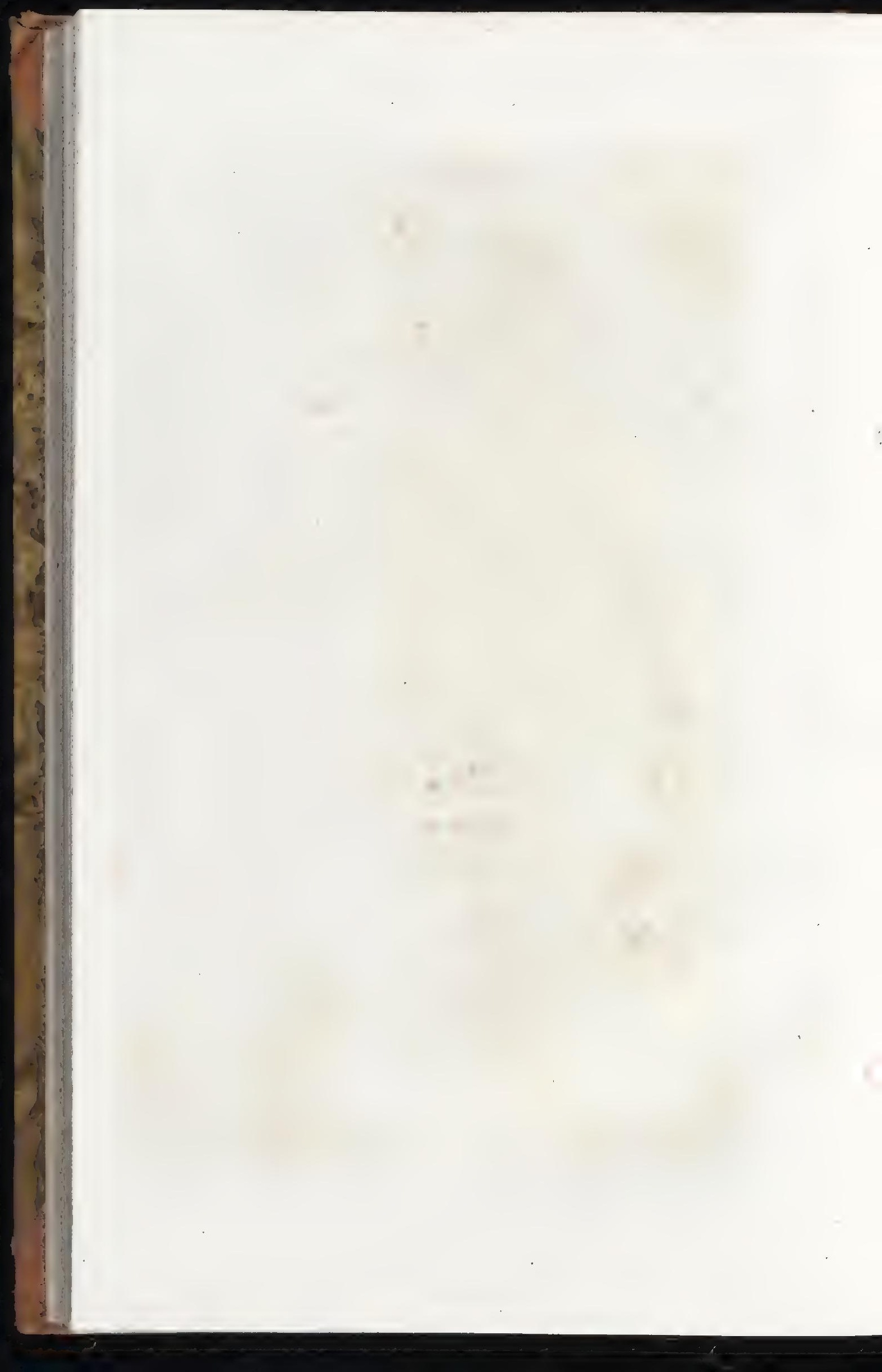


ment parfaitement, il suffit du moindre rayon de soleil pour entretenir une douce chaleur. On se garantit du froid des nuits par des paillassons qui garnissent les croisées en dehors, et par des contrevents en bois dans l'intérieur.

La construction des croisées est remarquable. Les fenêtres étant fort lourdes, elles auraient perdu leur aplomb si elles eussent pesé sur les gonds. On a prévenu cet inconvénient en plaçant les gonds en haut et en bas au quart de leur largeur, de manière qu'elles roulent sur deux pivots. Le mouvement est si doux qu'il suffit de les pousser du doigt pour les ouvrir, sans éprouver aucune résistance. Alors en s'appliquant contre le mur extérieur, elles y trouvent un loquet qu'elles abaissent, et qui en se relevant les maintient dans la même position, sans qu'elles puissent jamais être dérangées par le vent.

Les arbres qu'on abrite dans la serre tempérée sont originaires, les uns de l'Asie mineure, de la Grèce, de la Floride, et autres contrées de l'hémisphère boréal dont la température est à peu près celle du midi de l'Espagne: les autres viennent de climats aussi froids que celui de la France, par exemple de la terre de Diemen et de la Nouvelle-Zélande; et cependant ils périraient si on les laissait passer l'hiver en pleine terre. Nous croyons devoir expliquer ce phénomène 
280

DESCRIPTION DU MUSÉUM.

dont la raison n'est pas généralement connue : et ceci nous donnera l'occasion de faire remarquer que l'utilité d'une grande serre tempérée ne se borne pas à favoriser les progrès de la science en nous faisant connaître des végétaux étrangers, ou à satisfaire notre curiosité par la vue de plantes magnifiques différentes de celles qui croissent autour de nous; mais qu'elle peut rendre service à l'agriculture et embellir nos jardins en naturalisant dans notre pays des plantes agréables et des arbres étrangers.

On a souvent parlé d'acclimater les arbres des pays chauds en les accoutumant peu à peu à une température plus froide. Ce qu'on peut obtenir par ce moyen est renfermé dans des limites bien étroites. Un arbre que six degrés de froid font périr dans son pays natal, ne s'accoutumera jamais à en supporter dix chez nous. L'oranger, l'olivier, le figuier, sont cultivés en France depuis des siècles, et cependant ils ne résistent point à un hiver rigoureux, et presque tous les orangers et les oliviers sont morts en Provence dans l'hiver de 1820 .

Les arbres qui ne réussiraient pas d'abord chez nous et qu'on peut espérer d'y naturaliser, sont ceux qui ne supportent pas nos hivers parce que c'est en automne qu'ils entrent en séve, et que e'est pendant les mois d'hiver qu'ils fleurissent. 
Ainsi les eucalyptus, les banksia, les casuarina, croissent à la terre de Diemen où il fait aussi froid qu'en France; mais ils fructifient pendant les mois de janvier, février, mars et avril, qui correspondent à l'été et à l'automne de leur pays natal. Lorsqu'on en apporte des individus chez nous, ils conservent la même habitude, ils suivent les mêmes périodes pour le développement de leur végétation, et la gelée les fait périr. Mais en les cultivant dans une vaste serre tempérée on parvient à en obtenir des graines fécondes, et ces graines semées à une époque favorable donnent des individus dont quelques-uns entrent en séve au printemps ; alors on peut sans crainte les élever en pleine terre. Ce changement n'a pas toujours lieu dans la première génération ; mais en continuant la multiplication par semis, on est à peu près sûr du succès.

Nous citerons un exemple pour prouver ce que nous venons de dire. La belle-de-nuit à longues fleurs, aujourd'hui si commune dans nos jardins qu' elle parfume au coucher du soleil, fut en 1760 introduite au Jardin du Roi par M. Lemonnier, qui l'avait reçue du Mexique; elle fleurit au commencement de l'hiver, et l'on fut obligé de la renfermer dans la serre chaude. On en recueillit des graines qui donnèrent des pieds que l'on conserva dans l'orangerie : les graines de ceux-ci en 
donnèrent qui fleurirent au commencement de l'été, et c'est depuis cette époque qu'elle passe l'hiver dans nos jardins.

Les superbes dahlia du Mexique dont M. Cavanilles envoya des racines au Jardin du Roi au commencement de 1802 , furent placés à la fin de l'hiver dans une serre chande et sous châssis à une température de quinze degrés; cependant ils poussèrent fort tard et ne fleurirent qu'à la fin de l'automne. On les a multipliés de graine, et on est parvenu à les faire fleúrir en été et à en obtenir de très-belles variétés qui n'exigent plus aucun soin. M. Thouin avait annoncé ce résultat, mais il ne se flattait pas qu'on y parvînt en si peu de temps (I).

Nous ajouterons que les melaleuca et les métrosidéros, qu'on a élevés de graines, fleurissent plus tard que ceux qui nous ont été d'abord apportés de la Nouvelle-Hollande.

Ces observations nous portent à croire que notre serre tempérée enrichira un jour le sol de la France de plusieurs plantes agréables, et de plusieurs arbres utiles pour la marine et pour l'ébénisterie.

On place les caisses dans cette serre au mois d'octobre; on les en retire au mois d'avril ou au

(1) Voyez le mémoire sur les dahlia, Ann. du Muséum, tom. 3, p. $42 n$. 
commencement de mai, et elle se trouve alors entièrement vide. Vers le mois de mars elle offre un coup d'œil admirable, parce que la plupart des arbres qu'elle renferme sont alors en fleur; et que plusieurs d'entre eux sont plus grands qu'aucun de ceux de la même espèce qu'on puisse voir en Europe. On ne saurait se faire une idée de l'élégance et de la beauté des mimoses ou sensitives de la Nouvelle-Hollande, dont les unes sont couvertes de longs épis et les autres de houppes de fleurs diversement colorées (I). A ces mimoses se mêlent les sophora microphylla et tetraptera (2), des casses à fleur jaune, des sparmannia (3), des pittosporum (4), des camphriers, des

(1) Il y a dans la serre tempérée seize especes de mimoses arborescentes de la Nouvelle-Hollande; celle en panache, $m$. lophanta, et celle en grappe, $m$. botricephala sont les plus belles, du moins par leur feuillage; celles à fenilles simples, comme le floribunda, l'armata, le sophora, sont admirables lorsqu'elles sont en fleur. On peut espérer que les unes et les autres seront un jour cultivées en pleine terre dans les départemens du midi. Quelques mimoses sont du nombre des arbres qu'on emploie à la Nouvelle-Hollande pour les constructions navales et civiles.

(3) Tous deux originaires de la Nouvelle-Zélande, introduits d'abord en Angleterre en $177^{2}$ par sir Joseph Banks.

(3) Charmant arbrissean originaire du cap de Bonne-Espérance, introduit en France et cultivé au Jardin du Roi au commencement de ce siècle. Il est constamment couvert de superbes bouquets de fleurs depuis le commencement d'octobre jusqu'à la fin de mai. Nous sommes fondés a croire qu'en le multipliant de graine on retardera sa floraison. I. sera alors l'un des plus beaux ornemens de nos jardins.

(4) Pittosporum undulatum, Vent. H. Cels; et Pittosporum Tobira, Hort. Kew. Le premier est un arbrisseau dont les fleurs ont l'odeur 


\section{DESCRTPTION DU MUSEU.}

lauriers de Madère, des banksia, des hakea, des mélaleuca, des visnea, etc., et les deux palmistes dont nous avons déjà parlé. Au devant de ces arbres qui déploient librement leurs branches à vingt pieds au-dessus du sol, et dont : quelques-uns atteignent la voûte, sont des plantes ligneuses, des coronilles orientales, des indigotiers, une très-belle collection de géranium ou pélargonium, etc. Sur la tablette qui est au devant des croisées, on voit, comme dans l'ancienne orangerie, des pots où sont diverses plantes alpines qui fleurissent vers le mois de février.

Au commencement de mai les: arbres les plus remarquables de cette serre sont placés dans le rond qui est en face de l'amphithéâtre : on y voit des métrosidéros, des mélaleuca, des eucalyptus, des leptospermum, des banksia, des mimosa (I).

du jasmin; sa tige contient un suc qui, suintant à travers l'écorce, devient-concret et se présente sous la forme d'une poussière résineuse. Il nous a été apporté de Ténériffe par Riedlé. Le second est un arbre cultivé à la Chine à cause de la prodigieuse quantité de fleurs très-parfumées dont il se couvre en été. Il n'est connu en Europe que depuis quelques années.

(1) Les eucalyptus, les banksia, les mimosa ct les casuarina sont les plus grands arbres de la Nouvelle-Hollande, et ils y sont employés pour la construction et la mâture des vaisseaux. M. de la Billardière et M. Péron ont vu à la terre de Diemen, des eucalyptus qui avaient 160 à 180 pieds d'élévation, et dont le tronc avait 25 pieds de circonférence. Les sauvages creusent ces troncs avec le feu pour se faire des re. traites, et cela n'empêche pas qu'ils ne continuent de végéter. Voyez la Billardière, Voyage à la recherche de la Peyrouse, t. 1, p.13 $1 .-$ Péron, Voyage des découvertes, t, 1, p. 23z. Freycinct, idem, p. 4). 
Les doubles des mêmes individus, et ceux qui ne sont pas très-grands, sont rangés dans le parterre qui tientaux semis, le long d'un mur exposé au levant et bien garanti du nord et de l'ouest. Les autres arbrisseaux et les plantes vivaces sont disposés en amphithéâtre sur la terrasse qui est au devant de la serre, et la garnissent dans toute sa longueur. Derrière la serre du côté du nord et adossés contre le mur, sont au rez de chaussée des ateliers pour le Muséum, et au-dessus des logemens de jardiniers et un laboratoire, où l'on arrange toutes les graines qu'on a recueillies pour en faire des distributions.

Quoique cette serre soit fort grande et fort belle, sa construction pourrait être plus avantageuse pour le développement de la végétation. Il est à regretter qu'elle ne soit pas éclairée par le haut comme par la façade; dans plusieurs des arbres qu'elle renferme, les branches qui sont vis-à-vis des croisées, sont couvertes de fleurs; mais celles qui s'élèvent au-dessus, ou qui sont tournées du côté du mur, en sont entièrement dépourvues. On peut remarquer aussi, qu'en dirigeant tous leurs rameaux vers la lumière, les jeunes arbres perdent leur forme naturelle; ce changement est peu de chose dans l'oranger, mais il est très-sensible dans les mimoses, les eucadyptus, etc. 
§. IX: SERRES CHAUDES.

IL y a au Jardin cinq serres chaudes qui ont chacune une destination particulière. Nous allons les visiter successivement, en commençant par la plus grande qui est placée le long de la partie supérieure de l'école, et abritée du nord par la petite butte.

On y entre par une porte qui est vis-à-vis de l'allée des marroniers. On trouve d'abord une petite cour où sont rangées dans la belle saison les plantes les plus curieuses de la serre. A gauche une autre cour encore plus petite et fermée, où sont des couches et des châssis pour quelques plantes précieuses, qui dans leur première jeunesse exigent beaucoup de chaleur et des soins particuliers; et un cabinet, où le jardinier de la serre se tient pour faire les rempotages.

Cette serre est divisée en trois parties, ou plutôt elle est composée de trois serres distinctes, disposées en amphithéàtre et adossées les unes aux autres. La supérieure, ou celle du fond, à laquelle on monte par un escalier placé en dehors est fermée de deux portes, entre lesquelles est un 


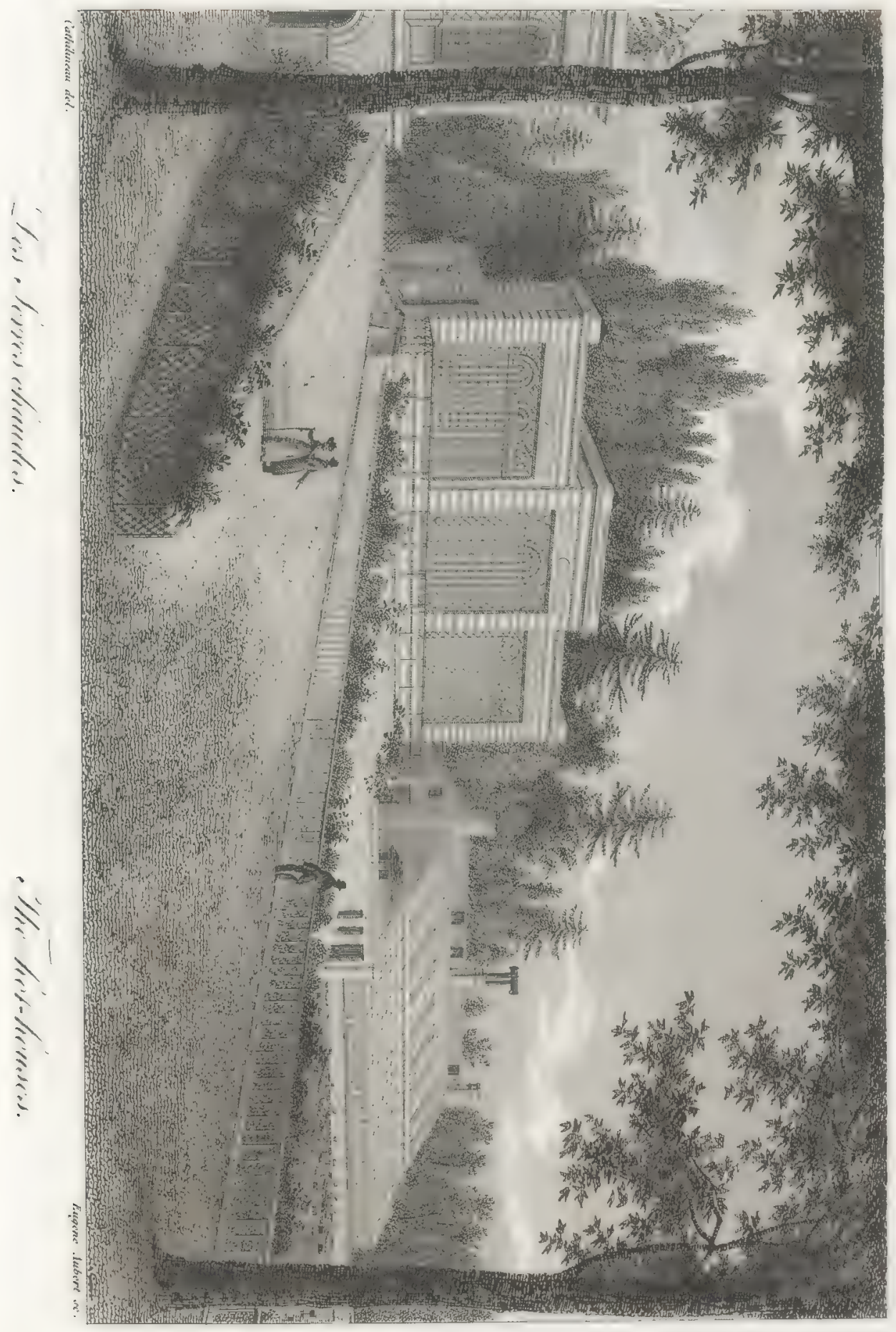




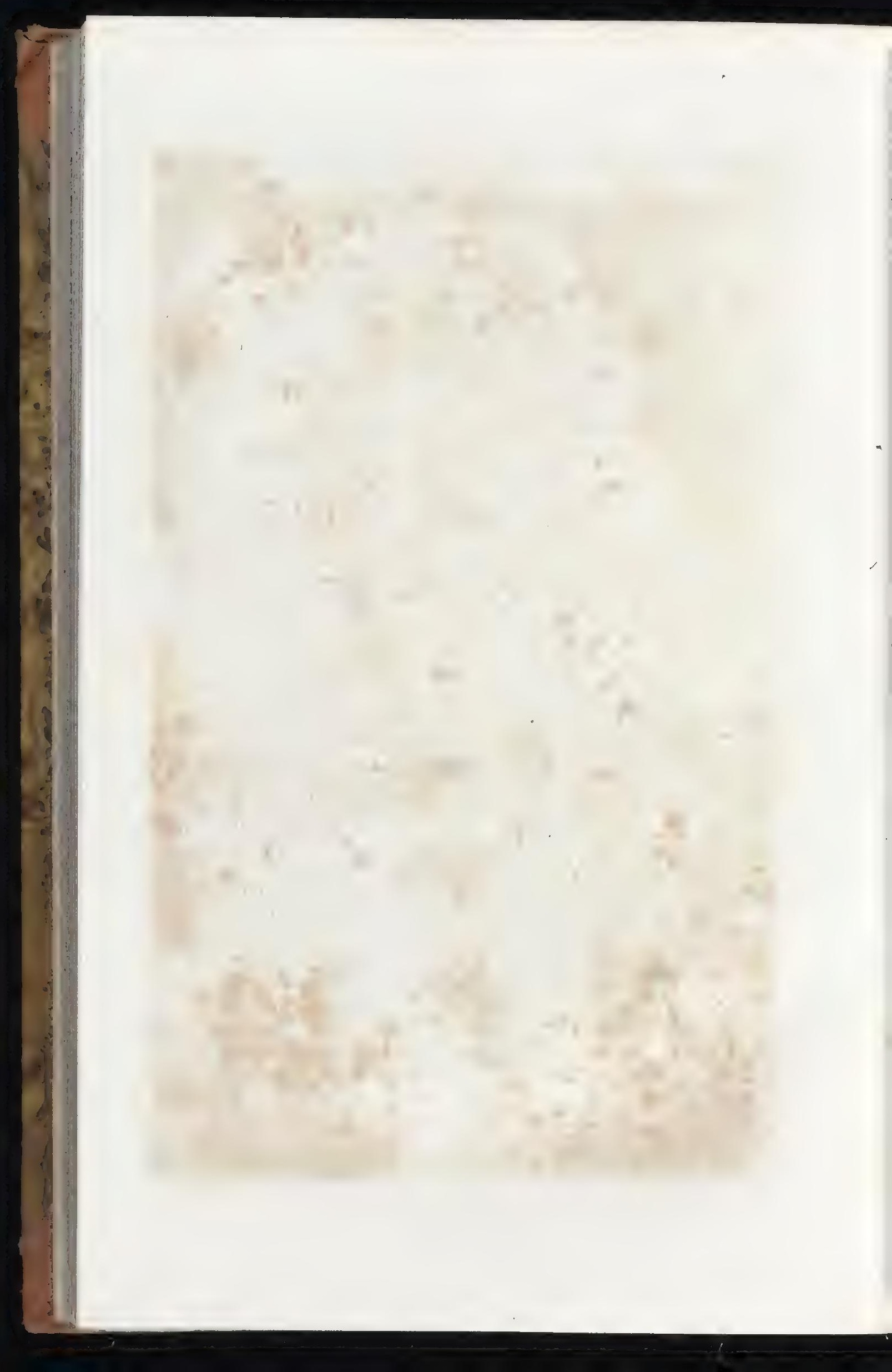


tambour. La seconde n'en est séparée que par un châssis vitré. Le sol de celle-ci est de cinq pieds plus bas, et le toit en est moins élevé. On y entre de plain-pied par le petit cabinet. La troisième serre n'a pas l'étendue des autres. Pour la placer au devant de la précédente, on a été obligé de prendre la moitié de la banquetic qui s'étendait entre l'école et le mur des serres, et sur laquelle on arrangeait pendant l'été les plantes qu'on avait retirées des serres. Elle communique par un escalier intérieur avec la précédente, et elle a une porte ouverte dans l'école de botanique.

Les toits des trois serres dont nous venons de parler sont en verre : on les couvre de paillassons soit pendant les grands froids, soit lorsqu'on craint un orage, et quelquefois lorsque le soleil est trop árdent.

La partie supérieure fut construite par Buffon en I 788, sur l'emplacement où se faisaient auparavant les semis pour lesquels on venait de disposer un nouveau local. Elle a i 25 pieds et derni de long, 12 pieds 4 pouces de large, et 15 pieds de haut, et le milieu est garni d'une conche de tannée dans laquelle les pots sont placés. Elle est. chauffée par quatre poêles. Elle fut d'abord destinée à recevoir une centaine d'arbres fruitiers (I)

(1) Des anones, des sapotilliers, des goyaviers, des longaniers, des Hanguiers. 
des tropiques qui avaient été envoyés au Jardin; ces arbres devaient y être placés non dans des caisses, mais sur le sol même, dans l'intention de les faire fructifier et d'en recueillir les graines pour propager et naturaliser les espèces qui pourraient être cultivées dans le midi de la France. Depuis cette époque, il nous est arrivé des pays chauds un si grand nombre de plantes, qu'on a été contraint de ménager dans cette serre assez d'espace pour les loger; on a en conséquence renoncé à y cultiver des arbres en pleine terre et à leur donner ainsi tout le développement qu'ils peuvent acquérir.

On trouve dans cette serre un grand nombre de végétaux fort rares et de la plus grande beauté. Nous nous contenterons de citer deux grands baquois ou pandanus odoratissimus; dont le tronc renflé vers le haut, et sillonné en spirale par l'impression des anciennes feuilles, pousse près de sa base des jets qui vont s'enraciner autour de hi, et le soutiennent comme des arcs-boutans ( 1 ) ; le ravenala (2), de la famille des bananiers, très-

(1) Ses fleurs mâles sont recherchées à cause de leur odeur ; on en met un petit faisceau dans les appartemens pour les parfumer, et on les vend en Égypte pour cet usage.

(2) Le ravenala s'eleve à la hauteur des palmiers; son trone nu est couronné par des feuilles, qui ont 6 à 10 pieds de longueur, sur 2 pieds de large, et qui sont disposées en éventail. Les Madegasses le nomment l'arbre des voyageurs, parce que les gaines des pétioles de ses feuilles forment un réservoir, toujours rempli d'une eau fraîche et limpide. 
utile aux habitans de Madagascar qui se servent de ses feuilles pour couvrir leurs maisons, et font de la farine avec ses graines, après avoir retiré de l'huile de la pellicule bleue qui les recouvre; le strelitzia du Cap, plante de la même famille, nouvellement introduite, et dont la fleur, en partie écarlate, en partie d'un beau bleu, a une forme si singulière qu'on ne peut la comparer à aucune autre; le caryota urens, espèce de palmier de l'Inde extrêmement rare, dont les feuilles pinnées ont des folioles triangulaires, découpées à leur bord; le littcéa, arbrisseau de la famille des narcisses, récemment apporté d'Italie par M.Bosc, et qu'on avait d'abord pris pour un yucca, parce que ses feuilles longues, étroites et pendantes ont comme celles de l'yucca filamentosa des fils blancs sur leurs bords; des pimento, ou toute-épice, espèce de myrte de la Jamaïque, qui doit son nom à l'odeur et à la saveur de ses feuilles et de ses rameaux; despsydium ou goyaviers; des eugenia jambos ou jamrose, dont le fruit est parfumé ; l'individu mâle du brucea ferruginea l'Hér. (I), apporté par Bruce de son voyage d'Abissinie; l'olivier à feuilles échancrées dont M. du Petit-Thouars a fait

(1) M. de Lamarck, qui l'a décrit le premier, lui avait donné le nom de brucea anti-dysenterica, parce que dans le pays où il croît, ses feuilles sont regardées comme un spécifique cuntre la dyssenterie, et que Bruce lui-même en avait fait usage avec succès. 
290 DESCRIPTION DU MUSEUM.

un genre sous le nom de noronhia, arbre superbe qui croît dans l'Inde et à Nadagascar, où l'on en mange les fruits; le mahogoni ou bois d'acajou ; des cecropia et des cocoloba, arbres des Antilles très-remarquables par la grandeur de leurs feuilles, qui dans le premier sont en bouclier, minces et argentées en dessous, et dans le second épaisses, coriaces, et d'un vert sombre; (I) le litchy de la Chine, dont les fruits du plus beau rouge sont excellens à manger; le citharexylum ou bois guitare, ainsi nommé parce qu'on prétend que son bois est propre à la fabrication des instrumens de musique; le sterculia fetida, grand arbre des Indes orientales, à feuilles digitées, dont les fleurs ont une odeur insupportable, mais dont les graines sont employées à faire de l'huile; le sapium des Antilles, aussi vénéneux que le mancenillier; le gardenia thunbergia, espèce à fleurs plus belles et plus odorantes que celle qu'on a jusqu'à présent cultivée sous le nom de jasmin du Cap; des roseaux de la canne à sucre violette d'Otaíti, espèce ou variété qui a l'avantage d'être beaucoup plus hâtive que celle de l'Inde; des begonia auxquels la forme et la couleur de leurs

(1) Le cocoloba uvifera porte aux Antillesle nom de raisinier, parce que ses fruits qui sont des drupes arrondis, d'une saveur très-agreable, forment des grappes parfaiteruent semblables aux grappes de raisin, quoique d'un volume plus considérable. 
leuilles donnent un port fort singulier; enfin de superbes figuiers de l'Inde, parmi lesquels on doit remarquer le ficus elastica, dont le lait donne de la gomme élastique, et le ficus macrophylla qui nous rappelle le souvenir du jardinier Riedlé, l'un des voyageurs qui ont procuréau Muséum le plus de plantes vivantes: Riedlé attachait tant de prix à cet arbre, qu'il avait trouvé à Timor, qu'en mourant il pria ses compagnons de voyage de ne rien négliger pour le conserver et le faire arriver en bon état au jardin du Muséum.

Cette serre est chauffée par quatre fourneaux; on a soin que le thermomètre de Réaumur y marque toujours au moins douze degrés. Elle est partagée en deux dans sa largeur par une cloison vitrée. La partie au-dessous est nommée serre Baudin, parce qu'elle fut construite en 1798 , pour loger les plantes apportées par le jardinier du Muséum, Riedlé, qui avait accompagné ce capitaine dans son voyage à Porto-Rico, à Saint-Thomas, etc. Elle est destinée à des arbustes et des plantes des tropiques. On y fait des boutures sous châssis, on y cultive les plantes herbacées les plus curieuses, et l'on y soigne dans leur première jeunesse beaucoup d'arbrisseaux qu'on transporte dans la serre supérieure, lorsqu'ils sont parvenus à une certaine grandeur. Cette serre dépasse un 
292 DESGRITION DU MUSÉUM.

peu celle qui est au-dessus, elle a 140 pieds de long, 9 pieds et demi de large, I I pieds de hauteur. Elle est chauffée par trois poêles. Les plantes n'y sont point rangées dans le milieu, mais des deux côtés. A gauche le long des vitraux est une tablette sur laquelle on met les liliacées, les narcisses et les orchidées qui demandent beaucoup de lumière. Au-dessous, dans un encaissement de r 5 pouces de hauteur et qui est rempli de tannée, sont d'abord les boutures sous châssis, puis des pots contenant des plantes de lazone torride, qui ne s'élèvent pas beaucoup ; de l'autre côté on voit une banquette semblable, et des gradins où sont rangés les arbustes et les plantes d'une plus haute taille.

On remarque entre autres dans cette serre, le xylophylla, arbrisseau singulier, en ce que ses fleurs très-nombreuses sont situées dans les dentelures qui bordent les feuilles; des crinum et des pancratium qui, lorsqu'ils sont en fleur, répandent l'odeur la plus suave; de superbes amaryllis, comme la belladone et la grenésienne; des orchidées au nombre desquelles est la vanille; des aroídes, telles que la colocase, l'arum pictum et le pothos crassinevia; de jolies sensitives, et le sainfoin oscillant des bords du Gange, ainsi nommé parce que des trois folioles qui composent sa feuille, deux sont toujours en 
mouvement; des banisteria, des bignones (I), des grenadilles qui serpentent le long de la cloison, et forment des guirlandes sous le toit; deux de ces grenadilles, passiflora quadrangularis et passiflora princeps (2), sont admirables par la grandeur et l'élégance de leurs fleurs, qui sont solitaires et bigarrées de diverses couleurs dans le premier, rouges et en longues grappes dans le second.

Parmi les arbres et arbrisseaux qui n'ont pas encore fleuri, parce qu'ils sont trop jeunes, et qu'on garde dans cette serre en attendant que l'étendue de leurs branches et la hauteur de leurs tiges exigent un local plus vaste, on peut citer comme dignes d'attention : le tamarin, dont il serait difficile de trouver ailleurs un aussi bel individu; le sablier, hura crepitans, dont le fruit est dans tous les cabinets; le tamnus elephantipes, apporté de la Cafrerie, par M. Delalande, et qui est si remarquable par l'énorme tubercule ciselé et mamelonné duquel partent les tiges; le carolinea princeps ou pachirier, que M. Bonpland

(1) Bignonia unguis-cati.

(3) Le passiflora quadrangularis a une telle force de végétation, qu'il pousse dans l'année des tiges de 50 pieds de long; ses fleurs durent peu, mais elles se renouvellent pendant plusieurs mois; il nous a donné l'année dernière des fruits de la grosseur d'un petit melon. Ces fruits sont bons à manger. Le passiflora princeps ne nous est connu que depuis trois ans. Il a été donné au Jardin par M. Cels, qui l'avait reçu d'Angleterre. 
294

DESCRIPTION DU MUSÉUM.

nous a apporté de Schoenbrunn, et le carolinea insignis venu de graines envoyées du Brésil par M. Auguste de Saint-Hilaire, deux arbres superbes et par leurs grandes feuilles digitées, et par leurs fleurs qui ont jusqu'à dix pouces de diamètre, et renferment une innombrable quantité d'étamines ; le baobab du Sénégal (adansonia baobab), que M. Perrottet a apporté de Cayenne, où il lui a été remis par M. Poiteau : quoiqu'il ait déjà 7 pieds de hauteur, et qu'il soit très-vigoureux, nous n'espérons pas le voir fleurir ; mais il excite la curiosité, parce qu'au Sénégal son tronc acquiert 25 à 30 pieds de diamètre, et que, d'après le calcul d'Adanson, il peut vivre au moins quatre mille ans; enfin l'araucaria ou pin du Chili, que nous avons également élevé de graines envoyées par M. de Saint-Hilaire. Il a déjà trois pieds de haut et va bientôt passer dans l'orangerie : quand nous en aurons reçu de nouvelles graines, nous essaierons de le propager dans le midi de la France, où il pourra, s’il y réussit aussi bien que dans son pays natal, offrir un jour les arbres les plus propres à la mâture des vaisseaux.

On entretient constamment dans cette serre une chaleur de quinze degrés, et plusieurs des plantes qu'elle renferme y passent l'année entière parce qu'on n'ose les exposer dans leur jeunesse à l'humidité et à la fraîcheur des nuits. 
On place sur la tablette des poêles, quelques liliacées et quelques cactus qui ont besoin de beaucoup de sécheresse.

A l'extrémité de cette serre est encore un cabinet vitré, correspondant à celui qui est à l'entrée. Ce petit cabinet offre une retraite très-commode lorsque le peintre attaché au jardin vient dessiner quelque plante qui est en pot dans la serre.

Les botanistes visitent cette serre avec beaucoup d'intérêt, parce qu'on y voit chaque année des plantes nouvelles, venues de graines recueillies par des voyageurs dans les régions équatoriales. Ainsi nous en avons eu beaucoup l'année dernière qui ont été envoyées de. l'Inde par M. Leschenault, du Brésil par M. A. de SaintHilaire; et déjà nous en avons vu lever plusieurs des graines apportées par M. Freycinet et par M. Delalande. Parmi ces plantes il en est qui sont arrivées depuis quelques années et qui cependant ne sont point indiquées dans le catalogue imprimé, parce que le professeur de botanique ne peut les déterminer que lorsqu'elles ont fleuri; mais un catalogue manuscrit indique au moyen d'un numéro, l'époque à laquelle on les a recues, le lieu d'où elles viennent, et le nom de celui qui les a envoyées.

C'est depuis le mois de novembre jusqu'au mois d'avril que ces serres sont entièrement garnies. Au 
296 DESCRIPTION DU MUSÉUM.

mois de mai on en retire presque toutes les plantes pour transporter un individu de chacune à la place qui lui est assignée dans l'école, et pour disposer les autres sur une banquette le long du mur en face de l'école, de manière qu'on peut facilement les voir et les étudier. Quelques-unes sont aussi placées dans les petites cours qui sont aux deux bouts.

Le soin de cette serre est depuis vingt-deux ans confié à M. Richer. Ce jardinier, fort instruit dans le genre de culture dont il est chargé, fait voir les serres aux personnes qui lui sont adressées par les professeurs de l'établissement. Comme il a des relations avec tous les amateurs et les pépiniéristes qui cultivent des plantes rares, il va s'informer chez eux s'ils ont reçu quelques espèces nouvelles, et il se les procure en leur donnant en échange, avec l'autorisation de M. Thouin, d'autres plantes qui leur manquent.

La troisième partie a été construite à la fin de l'été dernier I82 r, pour renfermer la belle collection que nous venions de recevoir de l'Inde et de Cayenne. On l'a nommée serre Philibert, parce que ce capitaine de vaisseau avait pris sur son bord M. Perrottet, jardinier du Muséum, qui après avoir recueilli la plupart des plantes dont elle est garnie ( $\mathrm{x}$ ), les a toutes, soignées pendant la tra-

(1) Plusieurs des arbres cultivés à Cayenne, et particulièrement ceux 
versée, et les a apportées au Muséum dans le plus bel état de végétation.

Cette serre a 75 pieds de long sur 12 de large, et ro de hauteur. Elle est chauffée par deux fourneaux. Il est remarquable que depuis sa construction, la partie de la serre Baudin à laquelle elle est adossée est devenue meilleure. La plupart des plantes qu'elle renferme n'étaient point au Jardin du Roi, plusieurs même n'avaient encore paru dans aucun jardin de l'Europe. La collection était composée de quatre-vingt-cinq caisses contenant cinq cent trente-quatre individus de 6 pouces à 6 pieds d'élévation, et cent cinquante-huit espèces différentes. Nous citerons comme les plus intéressantes, l'arbre à pain sauvage (artocarpus incisa), et la variété obtenue par la culture, dont le fruit, qui ne contient point de graines, est la nourriture ordinaire des habitans des îles de la mer du Sud (I).

Le jacquier (artocarpus integrifolia), dont le fruit tuberculeux a la forme d'un melon, et jus-

à épicerie, ont étẻ remis à M. Perrottet par M. Poiteau, auteur de plusieurs mémoires insérés dans nos Annales, et qui a succédé à feu M. Martin dans la place de directeur des cultures des habitations royales à la Guiane française.

(i) Les pieds d'arbre à pain sans graines, que M. Perrottet vient de nous apporter de Cayenne, sont des rejetons d'un individu que nous avions envoyé dans cette colonie en 1797 , et que $\mathbf{M}$. Martin, alors directeur des pépinières, y avait multiplié. 
298 DESCRIPTION DU MUSÉUM.

qu'à deux pieds de longueur. Comme il est rempli de grosses graines, il n'est pas aussi bon que celui de l'arbre à pain; mais on le mange dans les Moluques.

Le betel (piper betel), dont les Indiens font, avec la noix d'arec et un peu de chaux, une préparation qu'ils mâchent continuellement.

L'arec (areca faufel), arbre de la famille des palmiers, dont les fruits sont un objet de commerce dans l'Inde.

Le cyclantus bifolius, que M. Poiteau croit être un nouveau genre de palmiers; et plusieurs autres palmiers que nous ne connaissions que par les descriptions d'Aublet.

Des cocotiers.

Un raphia de Madagascar (raphia pedunculata, Pal. de Beauv.), qui donne le sagou, et dont les feuilles sont employées à faire des tissus.

Un rotang dont les feuilles ailées sont terminées par le pétiole prolongé en un filament garni d'aiguillons longs d'un pouce, et placés à distance comme les folioles.

Le muscadier (myristica aromatica).

Le virola sebifera de Cayenne, arbre voisin du précédent et dont les graines contiennent une substance dont on fait des chandelles.

Plusieurs variètés de cannellier, dont une, envoyée de Ceylan à Manille où M. Perrottetl'a prise, 
est par sa saveur et son parfum très-supérieure à celle qu'on cultivait à Cayenne.

Le quassia amara, arbre de l'Inde dont le bois d'une excessive amertume est employé en médecine.

Le couroupita guianensis, grand arbre qui est pendant presque toute l'année couvert de fleurs et de fruits. Ses fleurs sont belles et odorantes; ses fruits ont la grosseur et la forme d'un boulet de canon, et c'est sous ce nom qu'ils sont connus dans les cabinets.

Le carapa guianensis, arbre qui s'élève à 80 pieds, et dont les graines sont employées à fairr? de l'huile.

\section{Le cacao.}

L'omphalea diandra, arbrisseau de la famille des euphorbes, dont les rameaux grimpans s'élèvent au-dessus des plus grands arbres et retombent ensuite jusqu'à terre, et dont les fruits renferment des amandes bonnes à manger.

Le genipa americana, arbre de l'Amérique méridionale, dont les fleurs ont une odeur agréable, et dont les fruits contiennent un suc d'un violet foncé qui sert à la teinture.

Le butonic (barringtonia speciosa, Forst.), arbre des Indes orientales, de la famille des myrtes, remarquable par son port, par la gtandeur et par la beauté de ses fleurs. Ses fruits sont connus 
300

DESCRIPTION DU MUSÉUM.

dans les cabinets sous le nom de bonnets carrés.

Le morinda umbellata, arbre de l'Inde dont la racine est employée à teindre en jaune.

Le nerium tinctorium (ou wrightia, Brown), arbre des Indes orientales analogue au laurierrose, et dont les feuilles donnent une fécule semblable à l'indigo, et employée aux mêmes usages.

Le mabolo, (cavanillea philippensis, Lam. Ill.), dont on mange les fruits aux Philippines.

Un arbrisseau non encore connu qui paraît être un cookia, et qui par l'odeur de toutes ses parties est semblable à la badiane ou anis étoilé de la Chine, dont les graines sont employées à faire des liqueurs.

Enfin beaucoup d'autres plantes qui n'ont pas encore paru dans nos jardins, et dont on ne pourra déterminer le genre ou du moins l'espèce que lorsqu'elles auront fleuri.

Par leur construction et leur disposition en amphithéâtre, les serres que nous venons de décrire sont bien préférables à celles que nous allons bientôt visiter ; cependant elles ne répondent ni à la grandeur ni à la beauté de l'établissement. La serre Buffon ayant été bâtie il y a 35 ans, a souvent besoin de réparations. Comme elle n'a que I 5 pieds de hauteur, on est obligé d'élaguer les branches et de retrancher la cime des arbres qui sont dans des caisses, et plusieurs d'entre 
eux ne peuvent y fructifier. Les serres Baudin et Philibert sont très-bonnes; mais où placer les arbres qu'elles contiennent lorsqu'ils auront acquis plus de développement? Il faudrait que la serre supérieure eût 25 pieds d'élévation, et que les caisses y fussent arrangées de manière que le pied des arbres fût au niveau du sol. Ce serait le seul moyen de conserver, de multiplier et de rendre utiles pour les progrès des sciences les richesses végétales que les voyageurs nous apportent à grands frais des régions équatoriales. Le jardin du Muséum ne doit pas offrir moins de ressources que celui de Schœenbrunn. Dans un établissement aussi vaste, aussi varié, les diverses constructions ne peuvent être faites toutes à la fois; mais nous ne devons pas douter qu'on continuera de s"en occuper comme on l'a fait depuis plusicurs années, et le ministre, en demandant un plan pour de nouvelles serres, nous a fait espérer qu'on en commencerait bientôt l'exécution.

En sortant des serres Buffon, Baudin et Philibert par l'extrémité à l'ouest, on traverse une cour, on suit un conloir qui passe à côté de la petite butte, et l'on arrive à l'une des deux serres construites par du Fay, dont l'entrée est au haut de la rampe qui conduit de la partie supérieure à la partie inférieure du jardin.

Cette serre qu'on nomme serre des arbris- 
scaux, a $7^{5}$ pieds de long, 9 pieds de large, et r6 pieds de hauteur. Au moyen de deux fourneaux placés par derrière, on y entretient pendant l'hiver une chaleur de huit degrés. Elle est destinée à la culture des grands arbrisseaux des tropiques, qui y sont plantés dans des caisses rangées sur des gradins les unes au devant des autres. On y roit de très-grands individus du sideroxylon atrovirens, et du schotia speciosa (I), l'erithryna corallodendron, ou arbre de corail des Antilles, auquel on a donné ce nom à cause du rouge éclatant de ses fleurs et de ses graines; le globa nuutans, plante de la famille des balisiers, nouvellement introduite en France et très-recherchée à cause de ses belles grappes de fleurs.

La seconde serre de du Fay a les mêmes dimensions que la précédente : elle a aussi deux fourneaux; mais on se contente de ne pas y laisser descendre la température au-dessous de six degrés. Elle est destinée aux plantes grasses, la plupart originaires d'Afrique, telles que les aloès, les cierges, les grandes joubarbes, les agaves, les euphorbes. On y remarque l'euphorbe des Canaries, dont on est obligé de soutenir avec des . cordes les rameaux étalés et dépourvus de feuilles,

(1) Arbrisseau du cap de Bonne-Espérance, d'un très-bel effet lorsqu'il est couvert de ses fleurs en grappe et de couleur écarlate, qui maissent sur le bois comme dans l'arbre de Judée. 
et qui par cette raison et à cause de sa grandeur ne sort jamais de la serre; l'aloe ferox dont la tige est très-élevée; le cactus monstrosus dont la tige verte et mamelonnée ressemble par sa forme à une masse de stalactite. Les plantes de cette serre, excepté un individu qu'on porte à l'école, sont dans la belle saison rangées sur une terrasse qui se trouve ainsi décorée des aloès, des cierges et des opuntia ou raquettes. Nous avons l'espèce qui nourrit la cochenille, mais l'insecte qu'on nous avait cnvoyé avec la plante a péri depuis trois ans, et nous croyons qu'il n'existe plus en Europe.

En sortant de cette serre on en trouve une fort petite et fort étroite avec un seul fourneau. Elle est garnie de gradins sur lesquels on place pendant l'hiver le genre nombreux des ficoïdes, et autres plantes analogues venant du cap de BonneEspérance. Il suffit que la température n'y baisse pas au-dessous de trois degrés.

Après la sérre des ficoïdes et à droite, est une serre enfoncée et adossée à la montagne. C'est la plus ancienne du Jardin : elle a été construite en I714 du temps de Vaillant; et on l'a nommée serre du Cafier, parce que c'est là que fut élevé le premier pied de café envoyé du Jardin de Leyde à Louis XIV, et dont les graines ont fourni les pieds qui ont peuplé les Antilles. Elle est des- 
304 DESCRIPTION DU MUSÉUM.

tinée aux plantes les plus délicates de l'Inde et d'autres contrées de la zone torride, qui y sont disposées sur une couche de tannée, placée dans le milieu de manière qu'on en fait le tour. Sa longueur est de 34 pieds, sa largeur de 14, sa hauteur de 15. Elle est chauffée par un seul fourneau, et l'on y entretient constamment une chaleur de douze degrés. La toiture est en verre dans la moitié de sa largeur.

Quoique cette serre ne soit pas fort grande, c'est celle qui offre le coup d'oeil le plus pittoresque, parce que les arbres et arbrisseaux placés au milieu ont des formes singulières, et contrastent entre eux par la variété de leur feuillage.

On voit dans cette serre un très-beau cycas circinalis, apporté de l'île de France par Joseph Martin, arbre d'une forme très-singulière et dont la moelle est employée à faire une espèce de sagou qui sert de nourriture aux habitans de Madagascar; des plumeria ou frangipaniers; des hibiscus tiliaceus; le spaendoncea tamarindifolia, apporté d'Abissinie par Bruce; des palmiers dattiers; un aletris fragrans qui a 12 pieds de hant, chose remarquable dans la famille des liliacées; ses fleurs en pyramide répandent une odeur délicieuse pendant la nuit; un beau pandantus et un draccena marginata de Madagascar : presque tous ces arbres passent l'année entière dans la serre. 
Le mur du fond est tapissé de deux espèces de graminées arborescentes (I).

En avant et sur des tablettes placées le long des vitraux est une nombreuse collection de stapelia, plantes dont les fleurs et les tiges sont extrèmement singulières, et qui seraient plus généralement cultivées par les curieux, si elles n'avaient une odeur très-désagréable.

Nous arrivons enfin à la dernière serre adossée à la montagne. Elle est composée de trois parties dont les deux premières construites en 1717 , sous l'intendance de Fagon et à la sollicitation de Vaillant, portent le nom de serre du cierge; parce qu'elles sont séparées par un grand cierge du Pérou, au-dessus duquel on a construit une lanterne vitrée de 40 pieds de haut.

La troisième partie, qui est à l'extrémité, a été ajoutée après la mort de Buffon, et on lui a donné le nom de serre Saint-Pierre, parce qu'elle a été terminée en $179^{2}$, sous l'administration de ce dernicr intendant du Jardin : elle est, comme nous le verrons bientôt, d'une construction différente des deux autres auxquelles elle est réunie.

La porte d'entrée de la serre est en face du cierge dont la case forme un cabinet particulier

(1) L'une d'elles n'a pas encore fleuri ; l'autre est le panicum latifolium dont les tiges creuses fournissent aux sauvages de l'Amérique les tuyaux de pipe, ou calumets, qu'ils s'offrent réciproquement en signe d'amitié. 
où l'on se contente d'entretenir cinq degrés de chaleur.

Ce cierge, dont les racines occupent peu d'espace, est dans une terre qu'on n'arrose point; il pompe sa nourriture dans l'air atmosphérique par la seule succion de son écorce. Il a déjà 40 pieds d'élévation, et l'on peut être sûr qu'il n'atteint jamais cette hauteur dans son pays natal, où ses rameaux qui sont articulés seraient brisés par les vents. Il se couvre toutes les années de fleurs qui se fanent en vingt-quatre heures, mais qui se succèdent pendant un mois. Ces fleurs ressemblent beaucoup à celles d'un autre cierge originaire des Antilles, nommé cactus grandiflorus, mais elles ne sont point aussi belles et n'ont pas le même parfum ( $\mathrm{x}$ ).

La partie à droite du cabinet renferme des arbrisseaux des tropiques, cultivés dans des vases ou des caisses qui occupent le milieu de la serre, et d'autres plantes disposées en amphithéâire sur

(1) Ge cierge fut, en 1700, envoyé a M. Fagon par M. Hotton, professeur de botanique à Leyde. Il fut planté au Jardin du Roi n'ayant que 4 pouces de hauteur et 2 pouces de diamètre. Il devint bientôt si grand, qu'en 1713 , sa tige - ${ }^{3}$ élerant au-dessus de la serre dans laquelle il était placé, on fut obligé d'en brûler le sommet avec un fer rouge pour arrêter son accroissement. Cela ne l'empêcha pas de pousser des jets latéraux. En 1717 , M. A. de Jussieu en donna la description et la figure dans les Mémoires de l'académie des sciences. Il avait alors 23 pieds de hauteur et 7 pouces de diamètre. On prit ensuite le parti de construire autour de lui une cage vitrée qu'on exhausse a mesure qu'il grandit. 
des gradins. le long des vitraux. On y remarque entre autres, de beaux dracana draco, ou sangdragon des Canaries.

La partie à gauche est aussi garnie d'arbrisseaux, parmi lesquels on distingue de très-beaux pieds du draccena reflexa de l'île de France.

Ces deux parties de la serre sont voûtées, elles ont chacune 40 pieds de long, 9 ou ro de large, et I I de hauteur. Les deux fourneaux sont placés par-derrière, et les tuyaux de chaleur passent sous le plancher.

La troisième partie, ou serre Saint-Pierre, est couverte en verre comme la serre Buffon; elle a 36 pieds de long, ro de large, r 2 de hauteur. Elle n'a aussi qu'un fourneau; mais elle est plus chaude que les précédentes, parce que les pots y sont placés dans une couche de tanuée.

On voit dans cette serre le cycas de l'Inde, $c y$ cas circinalis, et celui du Japon, cycas revoluta (I); des crinum, des pancratium, des dianclla, des pitcairnia; un theophrasta, arbre trèsrare dans les serres et fort remarquable, en ce que ses longues feuilles verticillées forment au sommet du tronc une touffe qui a la forme d'un vase; le chamarops histrix; le sabal adansonii,

(1) Les Japonais mangent ses fruits, et retirent de son tronc un sagou très-nourrissant. Ils attachent tant de prix a cet arbre, qu'il est expressément défendu de le transporter hors du pays. 
ou palmier nain des marais; le rhapis flabelliformis, ou palmier éventail de la Chine; l'aloès à bords rouges de l'île de Bourbon; le latanier de la Chine. A droite de la porte d'entrée, il y a dans une caisse un pied de passiflora alata, dont les rameaux étendus sous le toit ont cinquante pieds de longueur, et sont pendant huit mois de l'année chargés de fleurs moins grandes, mais d'ailleurs trèssemblables à celles du passiflora quadrangularis. C'est encore dans la même serre qu'a fleuri et fructifié il y a trois ans une nouvelle espèce de cierge dont la fleur d'une couleur changeante et glacée d'or, est par sa forme et son éclat l'une des plus belles que l'on connaisse. M. Desfontaines, qui l'a décrite et figurée dans les Annales du Muséum, lui a donné avec raison le nom de cactus speciosissimus.

Dans la belle saison, les plantes retirées de la serre que nous venons de décrire, sont placées le long du mur et sur la terrasse qui est entre cette serre et celle des ficoïdes en face de la serre du cafier. 



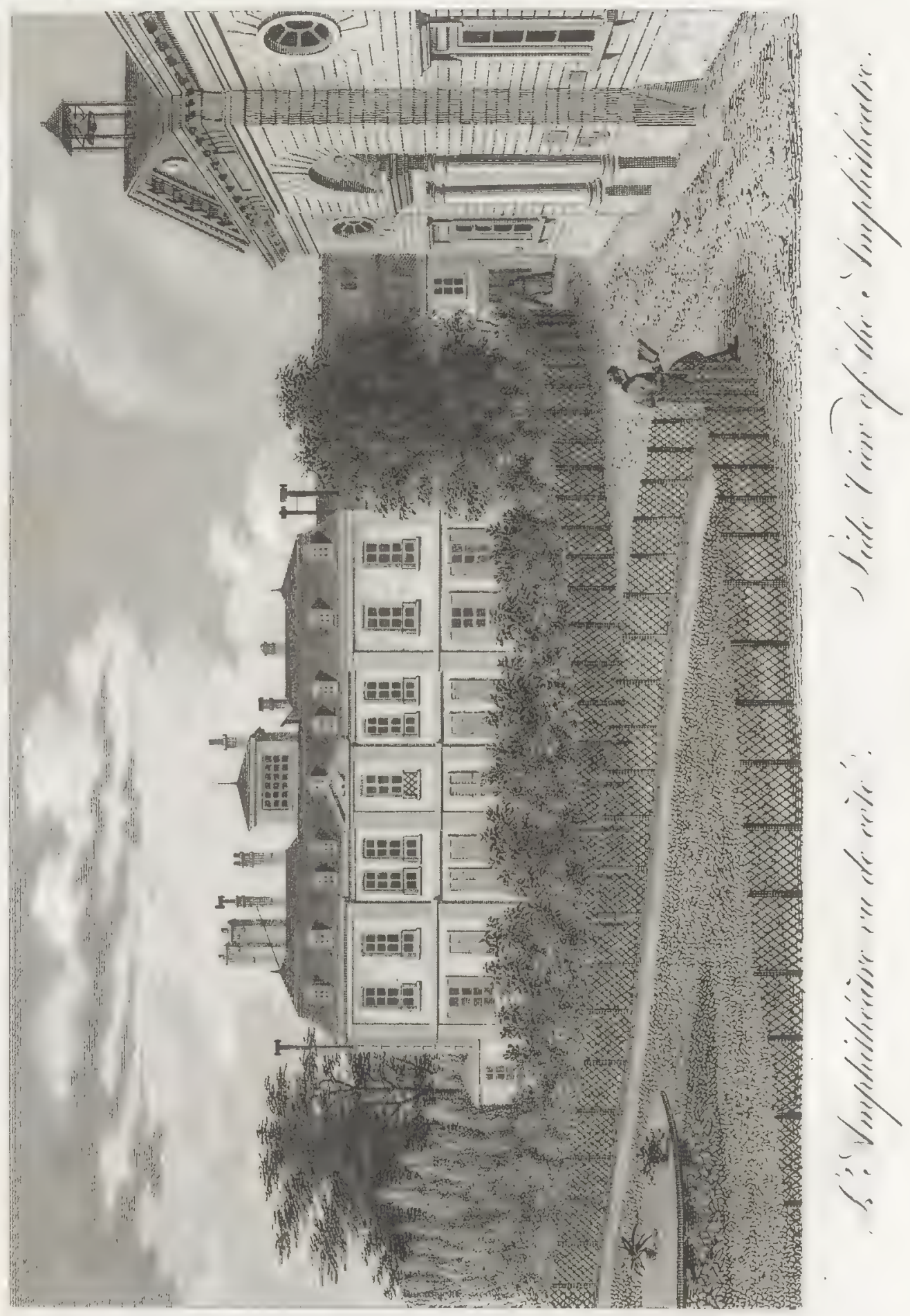




\section{CHAPITRE II.}

\section{§ I. GALERIE DE BOTANIQUE (I).}

Au-DEssus de la salle d'administration, et au premier étage, sont les galeries de botanique. On voit dans l'angle de l'escalier un tronc de palmier parfaitement cylindrique, de I 2 pieds de hauteur et ro pouces de diamètre, entouré d'une liane très-forte, dont les tiges aplaties, épaisses de 2 pouces, sont naturellement entre-greffées de manière à former un grillage. Ces tiges n'ont produit aucune impression sur le tronc, parce que l'accroissement du palmier (qui est monocotylédon), ne se fait point par couches concentriques. La pression d'une telle liane aurait causé des bourrelets dans tout arbre à deux feuilles séminales.

(1) Nous commençons la description des différentes collections par celle de la galerie de botanique, parce qu'il nous parait convenable de placer à la suite les uns des autres les objets qui ont entre eux le plus d'analogie. Or, la collection des plantes sèches est le complément de celles qu'on vient de voir dans l'école et dans les serres. Nous pàsserons ensuite au grand cabinet, ou nous verrons d'abord la minéralogie, puis la zoologie; de là nous nous rendrons au cabinet d'anatomie, puis à la ménagerie. Nons terminerons par la bibliothèque. 
A côté de ce tronc qui nous a été envoyé de Cayenne, en est un autre semblable et venu du même pays, mais coupé à dix-huit pouces de hauteur : le palmier est plus petit; mais la liane qui l'entoure est beaucoup plus épaisse. Cette liane est une espèce de figuier.

Plus haut, à côté de la porte d'entrée, est un tronc de chamærops, garni depuis la base jusqu'à la cime, de larges écailles, formées par la base des pétioles dont la partie inférieure est restée adhérente au tronc après la chute des feuilles.

En entrant, on tourne à droite et l'on passe successivement dans trois salles qui communiquent l'une à l'autre par une ouverture cintrée pratiquée dans le milieu. La première est la salle des bois, la seconde est celle des herbiers, la troisième celle des fruits : vis-à-vis de la porte d'entrée est une petite pièce qui sert de supplément à la salle des herbiers; enfin à gauche de la même porte est une autre salle partagée dans son milịeu par une cloison; et dont la dernière moitié est un cabinet de travail.

Parcourons ces différentes pièces. La salle des bois est éclairée par deux fenêtres, l'une au levant, l'autre au couchant, et garnie d'armoires vitrées dans toute sa longueur. Les trois premières armoires, c'est-à-dire les plus rapprochées de la fenêtre à droite, contiennent des échantillons 
qu'on a plus particulièrement choisis pour le cours de botanique. Ce sont divers exemples d'épiderme, d'écorce, de racines, de tiges, d'épines, de trachées, de moelle, de greffes, de bourrelets, de plaies, de broussins, et différentes coupes pour montrer l'organisation du bois. On y remarque une racine de polypodium barometz, Lin., ou agneau de Scythie (I); de beaux échantillons du liber ou écorce du boisà dentelle, les uns en rubans tenant au bois, les autres séparés; des tiges du bauhinia anguina, Roxb., dont la forme sinueuse ne se rencontre peut-être que dans celte seule plante (2); des troncs de hura crepitans et de zanthoxylum, qui ont de très-gros aiguillons; des tiges de cierge de huit pouces de dia-

(i) La racine, ou plutôt la base de la tige de cette fougère s'élève horizontalement au-dessus de la terre, et comme elle est revêtue d'un duvet soyeux très-épais, elle a la forme d'un petit agneau. C'est ce qui a fait raconter tant de fables sur ce végétal, qui, disait-on, se nourrissait des plantes dont il était entouré. Les échantillons qu'on voit dans les cabinets viennent du nord de la Chine. La plante n'a pas encore paru dans les jardins.

(2) Les tiges de cette singulière plante sont des bandes qui se courbent ou se creusent en une suite d'arcs, de manière que chaque portion a la forme d'un $\mathbf{S}$, et la tige entière celle d'une suite d'S placés les uns au bout des autres. Ces bandes ligneuses ont depuis i pouce jusqu'à 6 pouces de largeur, et depuis 3 lignes jusqu'à ı pouce d'épaisseur selon leur ancienneté. Les vrilles et les feuilles naissent sur la convexité des arcs, et la plante monte jusqu'au sommet des plus grands arbres. C'est le naga-mu-valli, de van Rheerle, hortus malabar., tom. 8. tab. 50 et 51 . 
mètre, couvertes de leurs longues épines en faisceau; un morceau de bois dans l'intérieur duquel on trouve l'impression de ce qui avait été écrit sur l'écorce en I $7^{50}$. Les lettres et les chiffres se voient encore sur l'écorce; mais il n'y en a pas la moindre trace sur les couches intermédiaires qui se sont formées entre l'écorce et le bois. On y voit aussi une corne de cerf sortant d'un tronc d'arbre dans lequel elle a été enveloppée lorsqu'il était encore jeune.

Les trois armoires suivantes, jusqu'à l'arcade, contiennent une collection des bois de l'Amérique septentrionale, apportée par M. Michaux fils, qui a donné l'Histoire des arbres de cette contrée.

Les échantillons sont bien conservés, et tous étiquetés avec certitude. Cette collection, la plus complète qui existe en ce genre, est d'autant plus intéressante, que tous les arbres dont elle se compose pourraient être cultivés en France, et que plusieurs d'entre eux seraient extrêmement utiles pour divers ouvrages de menuiserie et d'ébénisterie. Le bois du noyer noir, celui de l'érable rouge et de l'érable à̀ sucre, sont aussi beaux et aussi susceptibles de poli que ceux de l'Inde. Tous les morceaux appartiennent à des arbres qui avaient acquis leur grosseur moyenne, et plusieurs sont des planches de 8 pouces de largeur, sur 18 de hauteur. Il en est dont an a conservé 
l'écorce, lorsqu'elle offre des caractères particuliers.

De l'autre côté de l'entrée jusqu'à la fenêtre, au couchant, sont quatre armoires. Les deux premières sont réservées pour les monocotylédons; elles renferment des troncs et des coupes de palmiers, de baquois, d'yucca, de fougères arborescentes, de bambous, de rotang, de papyrus, etc., destinés à montrer la différence qui existe entre leur organisation et celle des arbres à deux feuilles séminales. On y verra un tronc de xanthorrea resinosa, arbre de la famille des asphodèles, qui donne une résine analogue au bray sec, et qui pourrait lui être substituée pour les usages de la marine (I); son bois, de couleur rouge et revêtu d'une écorce très-épaisse, est remarquable en ce qu'il paraît avoir des rayons médullaires trèsprononcés, tandis que, lorsqu'on l'examine attentivement, on y reconnaît l'organisation des autres monocotylédons.

La troisième contient des échantillons de bois indigènes. Ce sont des planches de 6 pouces sur 8 , et des coupes transversales.

Dans la quatrième, on voit une collection de bois de Cayenne propres à l'ébénisterie, étiquetés avec les noms qu'ils portent dans le commerce:

(1) V. le Voyage de découvertes aux Terres australes, par M. Freycinet, p. 41 . 
3I DESCRIPTION DU MUSEUM.

cette collection a été donnée au Muséum, par M. Jacob, ébéniste.

Les quatre armoires vis-à-vis, entre la fenêtre et le passage qui conduit à la seconde salle, renferment une collection des plus beaux bois étrangers employés dans l'ébénisterie et la marqueterie, avec les noms qu'on leur donnait autrefois. Cette collection faisait partie de l'ancien cabinet. La plupart de ces bois sont remarquables par leur couleur; il y en a plusieurs qui viennent de la Chine, et sur lesquels est écrit le nom chinois.

Des six armoires qui sont vis-à-vis celles dont nous avons parlé d'abord, les deux premières contiennent une collection des bois de PortoRico et de Saint-Thomas, apportée par Riedlé, jardinier du Muséum, au retour de son voyage avec le capitaine Baudin. Il y a presque toujours une coupe transversale et une longitudinale. On connait le nom des arbres qui ont fourni les échantillons; mais les bois ayant été avariés dans la traversée, ces échantillons sont très-imparfaits.

L'armoire à côté renferme cent sept échantillons de bois de Cayenne, envoyés par M. Duclerc (I). Ce sont des planches de 5 pouces sur 3 , polies d'un côté et brutes de l'autre. Tous sont

(1) M. Duclere est aujourd'hui administrateur des domaines du Roi à la Guadeloupe. 
nommés et partagés en séries de bois de charpente, bois de construction, bois d'ébénisterie, bois de teinture, etc.; ce qui rend cette collection extrêmement précieuse. Le bas de cette armoire et les deux armoires suivantes, contiennent d'abord des bois des îles de France et de Bourbon, dont les échantillons sont de la plus grande beauté, puis des morceaux de bois de divers pays et de différentes formes, remarquables soit par leur texture, soit par leur rareté, soit par leur usage. Enfin, dans la dernière armoire, celle qui est voisine de la fenêtre, on voit une nombreuse collection de toutes sortes de bois, apportée de Portugal, et donnée au Muséum en 1808 par M. Serrurier, frère du maréchal.

Cette collection serait plus utile si les échantillons étaient plus grands; ils ont la forme de livres in-r6, et n'ont que 4 pouces sur 3 .

Les bois étrangers renfermés dans les armoires, ne portent pas tous le nom de l'arbre auquel ils appartiennent, parce qu'il n'est pas toujours possible de les déterminer à la seule inspection, et qu'on ignore souvent à quel nom systématique répond le nom sous lequel ils sont connus dans le commerce. Quand les voyageurs envoient des échantillons de bois, il est à désirer qu'ils y joignent un rameau de l'arbre en fleur, pour qu'on puisse reconnaître le genre et l'espècè. 
La salle des herbiers, qui suit celle des bois, est garnie d'une boiserie formant des cases de Io pouces de hauteur, I I pouces de large, I 7 à I8 pouces de profondeur. Ces cases sont au nombre de trois cent quarante-quatre dans la partie de la salle qui est à droite de l'entrée, et de deux cent cinquante-six dans la partie à yauche. Des stores qu'on élève et qu'on baisse à volonté les garantissent de la poussière.

Les trois cent quarante-quatre à droite, renferment l'herbier général, qui est composé d'environ vingt-cinq mille espèces de plantes. Il y a ordinairement plusieurs échantillons de chaque espèce, soit parce qu'on n'a pas toujours des fleurs et des fruits sur le même échantillon, soit parce qu'on a eu soin de conserver un individu de la même plante recueilli dans différens pays. On a rapproché de l'espèce bien connue les variétés et les espèces très-voisines, qui n'en sont pas encore assez bien distinguées pour qu'elles soient décidément considérées comme eșpèces particulières.

Le fond de cet herbier est composé de l'ancien herbier de Vaillant, où toutes les plantes étaient étiquetées de sa main, avec la synonymie des auteurs connus de son temps, et l'indication du lieu où la plante avait été recueillie. Il y avait aussi dans cet herbier plusieurs plantes envoyées 
à Vaillant par des botanistes et étiquetées de leur main. Les écritures étant connues, lorsque ceux qui ont envoyé des plantes les ont publiées dans leurs écrits, on a un synonyme incontestable.

M. Desfontaines a joint à chacune de ces plantes sur une étiquette particulière, le nom systématique moderne le plus sûr et le plus connu. Les échantillons ont été comparés avec ceux des herbiers de M. de Lamarck et de M. de Jussieu.

Lorsque les botanistes qui publiaient des monographies de genres ou de familles sont venus visiter l'herbier du Muséum pour compléter leur' travail, M. Desfontaines les a invités à joindre aux échantillons des plantes qui n'a vaient point encore été décrites, les noms par lesquels ils les avaient désignées dans leur ouvrage. Ainsi plusieurs échantillons sont des types originaux, correspondans aux descriptions données par M. de Candolle dans les deux premiers volumes de son Regnum vegetabile; par M. Dunal dans la monographie des solanum; par MM. Bonpland et Kunth dans celle des mélastomes ; par MM. Mertens, Aghart et Lamouroux dans celles des fucus, etc.

L'herbier de Vaillant étant autrefois arrangé selon la méthode de Tournefort, ct renfermé dans des boîtes, n'était pas d'un usage commode. M. Desfontaines entreprit, en $\times 797$, de le disposer dans l'ordre des familles naturelles, avec des étiquettes 


\section{8 DESCRIPTION DU MUSÉUM.}

de genre, et d'y intercaler les autres plantes que possédait le Muséum. Il y joignit donc la magnifique collection que Commerson avait faite pendant son voyage autour du monde, et pendant son séjour aux îles de France et de Bourbon; celle que Dombey avait apportée du Pérou et du Chili; les plantes recueillies dans les îles de la mer du Sud, que Forster avait données à Buffon; puis un herbier envoyé de l'Inde par Macé, un de Cayenne par Martin; un de Madagascar par Chapelier; un des Antilles rapporté par.les botanistes qui avaient accompagné le capitaine Baudin, à Saint-Thomas et à Porto-Rico; un de SaintDomingue, donné par M. Poiteau; un de Java, donné par M. Leschenault; un de Perse, d'Égypte et du Levant, que MM. Olivier et Bruguière avaient fait pendant leur séjour dans ces contrées.

Le voyage à la Nouvelle-Hollande nous ayant procuré un grand nombre de plantes nouvelles, elles ont été également réunies à l'herbier général, qui a été encore enrichi par les dons que nous ont faits un grand nombre de voyageurs et de botanistes étrangers, et principalement M. Robert Brown.

Comme il est essentiel que l'herbier général ne soit pas trop volumineux, on a réservé les doubles des plantes qu'on a reçues, pour faire des 
GALERIE DE BOTANIQUE.

herbiers particuliers. Ainsi nous en avons un de la Nouvelle-Hollande, un de Cayenne, un des Antilles, un du Cap, un de l'Inde, un des îles de France et de Bourbon, un d'Égypte, un du Levant, etc. Ces herbiers, utiles pour connaître les plantes qui croissent dans tel ou tel pays, le sont surtout aux personnes qui travaillent à des monographies. On ne saurait se permettre d'analyser les plantes du grand herbier, qui doivent être conservées intactes : mais les doubles qu'on a mis à part, peuvent en cas de besoin offrir des échantillons que le professeur permet d'examiner en détail, soit pour les décrire, soit pour les dessiner. Ces doubles servent aussi à faire des échanges.

Nous avons encore d'autres herbiers spéciaux, desquels nous n'avons pris que les doubles pour les joindre à l'herbier général. Ce sont ceux qui servent de type à un ouvrage imprimé : tel est celui de Michaux père, où l'on voit toutes les espèces décrites dans sa Flora boreali americana; celui de M. Michaux fils, qui offre les échantillons des arbres de l'Amérique septentrionale, dont il a donné l'Histoire; celui des plantes de la Tauride et du Caucase, décrites par M. Marshall, et qui nous ont été apportées par son collaborateur M. Stevens; celui des plantes de France, que M. de Candolle nous a donné pour servir de type 
à la nouvelle édition de la Flore française (I) ; tel est enfin celui dont M. de Humboldt a fait présent au Muséum, qui renferme toutes les espèces recueillies dans son grand voyage, et publiées de concert avec lui par M. Bonpland, dans les Plantes équinoxiales, et par M. Kunth dans le Nova genera et species, etc. Cet herbier est d'environ quatre mille plantes, dont les trois quarts sont nouvelles.

Nous avons conservé religieusement l'ancien herbier de Tournefort, dans l'ordre où il était disposé, par respect pour la mémoire du fondateur de la botanique en France, et parce qu'on y trouve étiquetées de sa main ou de celle de Gundelsheimer, presque toutes les plantes qu'il avait recueillies dans son voyage au Levant, et qui ne sont indiquées que par une phrase dans le Corollarium institutionum rei herbaria.

Les plantes uniques de ces divers herbiers, qu'on n'a pas cru devoir transporter dans l'herbier général, sont pour le moins au nombre de six mille , ce qui, réuni à celles du grand herbier, fait plus de trente mille espèces bien distinctes. De l'aveu de tous les botanistes qui ont visité les principaux cabinets d'histoire naturelle de l'Eu-

(1) Au moment oủ nous écrivons, nous n'avons pas encore reçu ce dernier herbier; mais M. de Candolle nous a écrit qu'il l'avait complété et arrangé, et qu'il nous le ferait passer au premier jour. 
rope, il n'existe nulle part une collection aussi nombreuse; et cependant elle s'accroît tous les jours, et par les envois des voyageurs, et par les échanges ( $\mathrm{I}$ ).

Dans cette même salle qui a quatre croisées, deux à l'est et deux à l'ouest; l'un des trumeaux est occupé par une belle collection de champignons imités en cire, donnée au Muséum par S. M. l'empereur d'Autriche; l'autre par des modèles de fruits étrangers en cire ou en plàtre. Au plafond sont attachées des feuilles de corypha umbraculifera, qui ont I 5 pieds de diamètre.

(1) Dans l'année 1821 , nous avons reçu : $1^{\circ}$ un herbier de huit cents plantes, que M. d'Urville a recueillies sur les bords de la mer Noire, et dont il vient de publier le catalogue avec la synonymie des espèces connues, et la description de celles qui ne l'étaient pas; $2^{\circ}$ un de douze cents plantes de la Russie orientale, donné par M. Fischer, directeur du jardin de botanique de Gorenki près Moscou, soigneusement étiquetées avec les noms qu'elles portent dans les descriptions qu'il en a publiées ou dans le catalogue imprimé; $3^{\circ}$ un herbier de trois cents plantes des bords du golfe de Saint-Laurent, recueillies et données par M. de la Pylaie; $4^{\circ}$ un de la Martinique envoyé par M. A. Plée; 5० un des Philippines et de la Guiane apporté par M. Perrottet; $6^{\circ}$ un de la Cafrerie par M. Delalande ; $7^{\circ}$ de belles plantes du Brésil envoyées par le P. Leandro di Sacramento ; 80 enfin les plantes que M. Gaudichaud a recueillies pendant son voyage avec le capitaine Freycinet, et qui ont été données au Muséum avec toutes les autres collections d'histoire naturelle, par le ministre de la marine, M. Ie baron Portal. Les plus intéressantes de ces plantes seront décrites et figurées par M. Gaudichaud dans la relation du voyage que doit publier M. Freycinet. Nous attendons cette année des collections non moins considérables de l'Inde, du Sénégal, de la Guiane et du Brésil. 
La dernière salle, qui est celle des fruits, a cinq croisées au nord, et vingt armoires vitrées, dont quatre dans les trumeaux. Douze de ces armoires renferment des fruits desséchés ou conservés dans l'esprit-de-vin; les autres, diverses productions du règne végétal dont on fait usage dans la médecine ou dans les arts. Les fruits sont disposés selon la méthode que M. de Jussieu a adoptée pour la série des ordres naturels (I).

Pour suivre l'ordre de la collection, il faut en entrant se diriger à droite vers l'angle de la fenêtre, et faire le tour de la salle.

Les deux premières armoires sont destinées aux fruits des palmiers et d'autres végétaux monocotylédons. On y remarque de beaux cocos des Maldives (2), dont un a quatre lobes, et un en-

(1) Les fruits de la même famille se trouvent toujours dans la même armoire : mais les espèces et les genres ne sont point placés les uns à la suite des autres selon l'ordre d'affinité, parce qu'il a fallu avoir égard au volume des objets pour ménager l'espace, et pour les arranger de manière à ce qu'on pût les bien voir.

(2) Ce fruit a été anciennement nommé ainsi, parce qu'on le trouvait flottant sur la mer aux environs des Maldives, et que le souverain de ces îles s'en attribuait la propriété : on en faisait des vases qu'on vendait fort cher, et l'on se livrait sur son origine aux conjectures les plus bizarres. Ce fut en 1768 que Commerson décourrit aux îles Séchelles l'arbre qui le produit : il le nomme lodoicea. M. de la Billardière en a donné, dans les Annales du Muséum, tome 9, une description, faite aux îles Séchelles par M. Lillet, à laquelle il a joint ses propres observations : et il lui a conservé le nom de lodoicea en y joignant l'épithète Sechellarum. 
veloppé de son brou, chose assez rare dans les cabinets, et les chatons mâles de cet arbre, qui sont de la grosseur du bras; le doum, ou palmier de la Thébaïde, diverses espèces de coco; de très-beaux pandanus de l'Inde; des lontarus; des spadices de xanthorrea resinosa; un grand régime de maripa; des cycas; des nélumbo, etc. Les deux armoires à côté renferment des fruits de la famille des lauriers, parmi lesquels on distingue la noix muscade parfaitement conservée avec son macis, et d'autres espèces analogues non moins curieuses, et employées également à des usages économiques. Viennent ensuite de superbes fruits de diverses espèces de banksia, qui ont été achetés en Angleterre, et qui avaient servi de modèle aux belles figures données par White, dans son Voyage à la Nouvelle-Galles. Enfin des fruits très-remarquables et très-rares de la famille des bignones, des apocynées et des sapotilliers; tels que des cerbera, des strychnos, le nerium tinctorium de l'Inde, l'omphalocarpum, de M. Palissot de Beaurois, etc.

Les huit armoires placées à droite et à gauche de l'entrée, vis-à-vis des fenêtres, renferment toute la suite de la collection des fruits. Les quatre premières offrent les familles qui suivent les sapotilliers jusqu'aux légumineuses inclusivement. Plusicurs fruits de rubiacées, de savonniers, d'o- 
324 DESCRIPTION DU MUSÉUM.

rangers (1), de malvacées, de tiliacées, de myrtoïdes, appellent l'attention par leur forme et leur grosseur. On y remárquera surtout: le feronia elephantum, Roxb.; des bombax, ou coton de Mahot; l'ochroma lagopus; des baobab, ou pain-desinge; des capsules à cinq loges séparées par des membranes, renfermant des graines entourées d'un coton très-fin que les Indiens réservent pour faire les coussins qu'ils placent sous les statues de leurs dieux (2); des fruits de sterculia balanghas, composés de leurs cinq capsules ouvertes et garnies de leurs graines; l'espèce de cacao décrite par M. Bonpland; le carolinea insignis; le bertholletia, dont les graines étaient anciennement connues sous le nom de chàtaignes du Brésil, sans qu'on eût la moindre notion de l'arbre qui les produit, avant le voyage de MM. Humboldt et Bonpland qui en ont donné la description (3); différens fruits de lecythis, qui ressemblent à un vase dont le couvercle se sépare, ce quia fait donner à ceux d'un gros volume le nom

(1) M. Poiteau nous a donné les orangers qu'il a décrits dans son ouvrage; ils occupent le bas de la première armoire.

(2) Ce fruit nous a èté envoyé de Pondichéry par M. Leschenault, Les Indiens le nomment konna-marum; il appartient à un nouveau genre dont on trouve des espèces dans l'Inde et dans l'Amérique méridionale, et que M. Kunth a décrit sons le nom de cochlospermum.

(3) M. Bonpland n'arait pas vu les fleurs; M. Poiteau les à observées, et vient de nous les rapporter en herbier et dans l'esprit-de-vin : 'elles sont semblables à celles des lecythis, et prouvent l’affinité des deux genres. 
de marmite de singe; le couratari de la Guiane; le butonica, qui a la forme d'un bonnet carré.

Les fruits de légumineuses, qui occupent la par. tie inférieure de la troisième armoire et toute la quatrième, sont en très-grand nombre: on y verra les gousses de l'eperua falcata, du bauhinia racemosa, de l'hymencea verrucosa; celles du moringa, ou noix de ben, qui diffèrent de toutes les légumineuses, en ce qu'elles sont à trois valves; et celles du mimosa scandens, et du mimosa entada qui ont 4 à 5 pieds de longueur, quoiqu'elles soient sorties d'une fleur extrêmement petite; enfin, diverses graines d'une couleur éclatante, et qu'on emploie pour faire des colliers ou d'autres ornemens.

Les deux armoires à gauche de l'entrée, contiennent des fruits de la farnille des orties, de celle des térébinthes et de celle des cucurbitacées.

On y roit une collection de noix recueillies en Amérique sur toutes les espèces de noyers connus, et rapportées par M. Michaux fils; ces noix sont présentées dars tous les états par les coupes qu'on en a faites, et rangées avec beaucoup d'ordre: on y voit aussi une collection de courges, donnée par M. Duchêne qui avait fait un beau travail sur cette famille ( $\mathrm{I}$ ); des noix d'acajou avec le brou charnu sur lequel elles sont implantées; des

(1) Les dessins de M. Duchêné ont été acquís pour la bibliothèque du Muséum. 
pommes de mancenillier; le sablier (hura crepitans), qu'on est obligé d'entourer d'un fil de fer pour qu'il n'éclate pas; l'ambora, qui montre le passage entre la figue et la mûre; le jacquier, et les fruits de toutes les espèces d'arbre à pain, desséchés et conservés dans l'eau-de-vie, etc.

L'armoire suivante renferme une collection de cônes de pins et de sapins, dont la plupart ont été recueillis dans l'Amérique septentrionale, par M. Michaux; et de beaux échantillons de l'araucaria, ou pin du Chili, apportés par Dombey.

La dernière armoire du même côté, est destinée aux fruits qui appartiennent aux genres non encore classés dans les familles naturelles, et que les botanistes nomment incertoe sedis, et à ceux qui ayant été envoyés par des voyageurs, sans qu'on ait pu savoir de quelle plante its proviennent, ne sont pas encore bien connus.

Ici se termine la collection des fruits. On n'y a fait entrer que ceux d'une certaine grosseur et qui ne peuvent être conservés dans l'herbier; les petites graines exigeraient un nombre prodigieux de bocaux, et conséquemment beaucoup d'espace, et ne pourraient être vues qu'autant qu'on ouvrirait les bocaux dans lesquels elles seraient renfermées. M. Thouin en possède une collection très-nombreuse, et qu'on se propose 
de réunir dans cette même salle; mais il faudra pour cela l'arranger dans un meuble à tiroirs, où chaque espèce occupera une case particulière.

Les fruits de la collection sont étiquetés, et l'on a eu soin d'indiquer la figure de Gaertner, pour tous ceux qui ont été décrits par ce savant carpologiste. On trouve souvent, dans le bocal ou dans le carton où ils sont placés, des notes données par les royageurs, sur les usages auxquels ils sont employés dans le pays où ils ont été recueillis (I).

Les huit armoires suivantes, dont quatre jusqu'aux fenêtres et quatre dans les intervalles, renferment l'ancien droguier du Jardin du Roi, avec les additions qui y ont été faites.

Dans la première sont les racines, dans la seconde, les écorces; dans la troisième, les feuilles et les fleurs, etc., qui appartiennent à la matière médicale ou à l'économie domestique, soit en France, soit dans les pays étrangers; dans la qua-

(1) La collection des fruits s'est récemment accrue dans une proportion encore plus considérable que celle des plantes en herbier; parce que depuis les belles observations de Gaertner, les botanistes voyageurs mettent plus d'importance à se les procurer. M. Freycinet nous en a apporté beaucoup. M. Leschenault, M. Poiteau et $M$. Plée nous en ont eavoyé de très-rares, le premier, de l'Inde, le second, de la Guiane, le troisième, de la Martinique; et plusieurs amateurs d'histoire naturelle, qui en possédaient de fort curieux, ont bien voulu les donner au Muséum. Nous avons soin de mettre sur les étiquettes des divers objets, le nom de la personne qui en a enrichi la collection. 
trième, on a réuni quelques objets de curiosité, comme de belles tiges de graminées ( $\mathrm{r}$ ), des spathes de palmier, un régime de sagus, du guy sur une branche de chêne, de la toile non tissue, fabriquée dans les îles de la mer du Sud, etc.

Les deux premières armoires entre les fenêtres, renferment les gommes et les résines employées dans la médecine et dans les arts; la troisième, diverses préparations de végétaux, comme les indigo, les cachou, les parfums, des bougies faites avec la cire du myrica cerifera, une fiole du poison avec lequel les indigènes de Java empoisonnent leurs flèches; la dernière est destinée à des substances végétales qui ne sont pas bien connues.

La plupart des objets dont nous venons de parler sont dans des bocaux : un grand nombre viennent de la Chine; ceux qui composaient l'ancien droguier du cabinet, conservent les noms qu'ils portaient du temps de Vaillant et de Geoffroy; on y a joint le nom classique moderne. Sur une des tables qui occupent le milieu de la salle, est un vase de I 9 pouces de haut, et de 18 pouces de diamètre, formé d'un tronc de palmier creusé dans son milieu; ce vase nous a été donné par M. Maugé, qui l'avoit acquis à Porto-Rico, lors de son voyage avec le capitaine Baudin.

(1) Arundo sagittata, Persoon :Gynerium, Humb. et Gonpl. 
Nous avons dit qu'à gauche de la porte d'entrée des galeries, est une salle coupée en deux par une cloison. Le fond de la première partie est garni de cases où l'on garde l'herbier de Tournefort. A côté de la fenêtre sont deux régimes de sagus farinacea de 6 pieds de hant, et tout couverts de fruits ( I). On y trouve aussi des troncs de palmiers et de fougères, et des coupes de bois qu'on n'a pu placer dans les armoires, et qui présentent quelque chose d'intéressant : par exemple, une tranche d'un orme abattu. en I784, sur laquelle on observe, en comptant les couches annuelles, que celle qui répond à l'année I 7og, a été presque détruite par l'effet de la gelée.

I.e cabinet de travail qui est à la suite, est garni d'armoires vitrées qui renferment des fleurs et des fruits parfaitement conservés dans une liqueur spiritueuse, et une collection de gnaphalium et de xeranthemum ou immortelles du Cap, formant des bouquets et conservés secs dans de grands bocaux de verre. On y voit aussi deux cadres où sont des échantillons de la filasse retirée des diverses plantes textiles (monocotylédones et dicotylédones), cultivées au Jardin du Roi.

Les galeries de botanique ne sont point ouvertes au public, parce qu'elles n'offrent rien

(1) L'un nous a été envoyé de l'Inde depuis plusieurs années ; l'autre nous a été donné par M. Fulchiron. 
qui puisse intéresser les personnes qui n'ont aucune notion de l'histoire naturelle. Des herbiers dans des cases, des morceaux de bois les uns revêtus de leur écorce, les autres en petites planches pour en montrer la texture; des fruits desséchés, ou conservés dans l'esprit-dè-vin, sont des objets très-précieux pour l'étude, mais qui ne frappent pas d'abord les yeux. Il en est de ce cabinet, comme de celui d'anatomie comparée; mais s'il n'y a pas de jour où le public soit introduit, il n'en est aucun où les galeries ne soient ouvertes aux amateurs qui veulent les visiter, et les personnes qui désirent faire des recherches sur des genres ou des espèces, ou comparer leurs herbiers avec ceux du Muséum, y trouvent toutes les ressources possibles. Les monographies et les ouvrages descriptifs de botanique, publiés depuis quelques années, offrent la preuve de l'empressement qu'on met au Muséum à communiquer tout ce qu'on possède, et à donner toutes les explications qui peuvent favoriser les progrès de la science. 



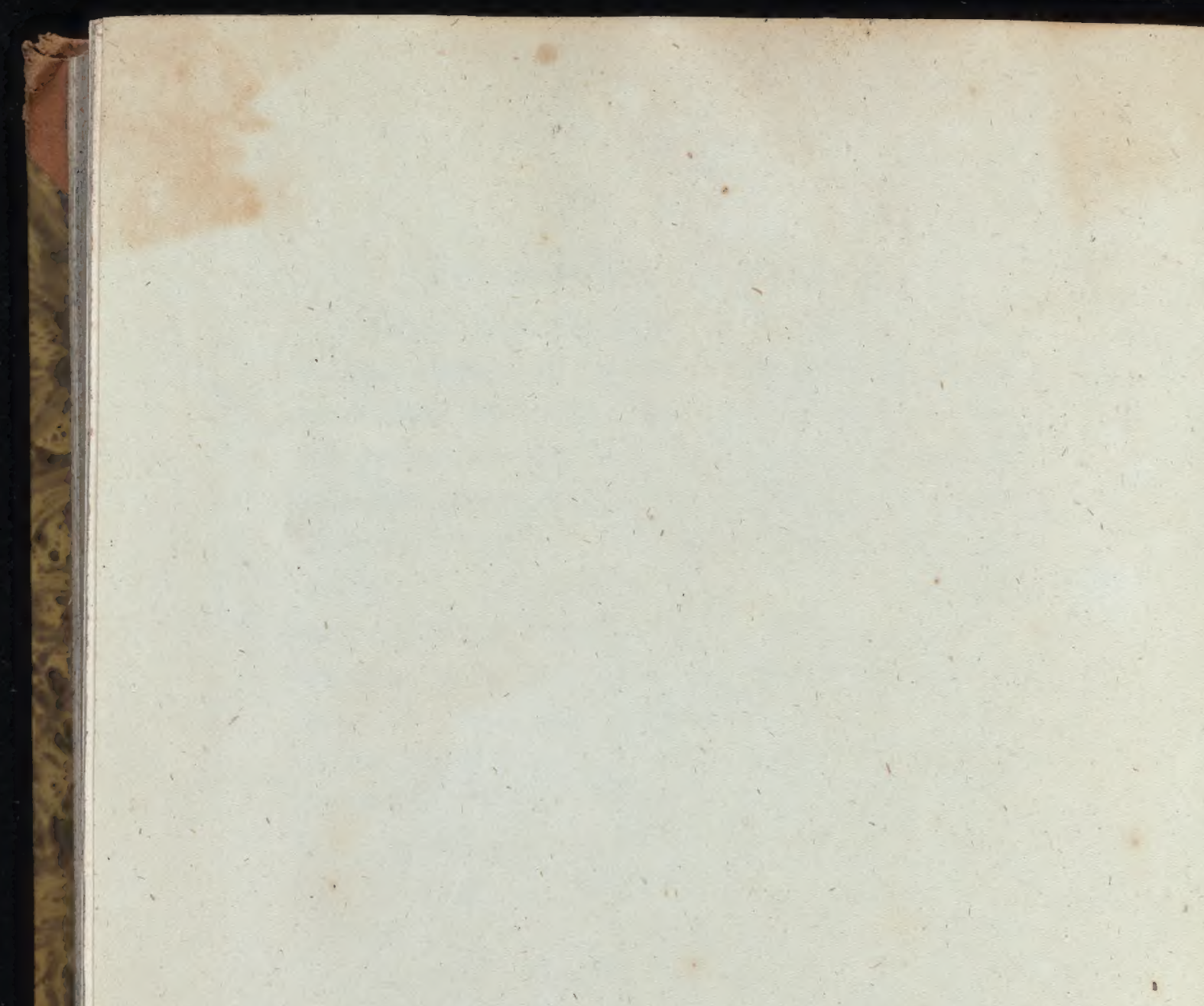





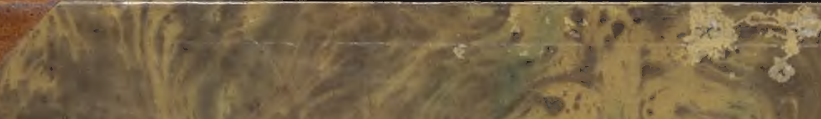

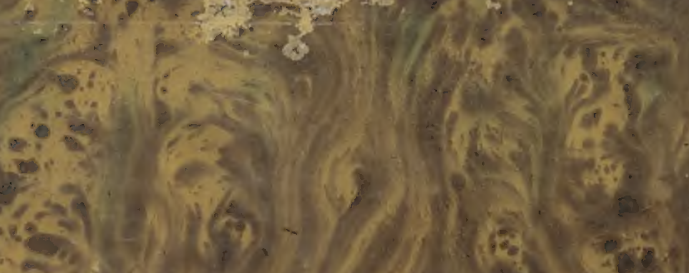

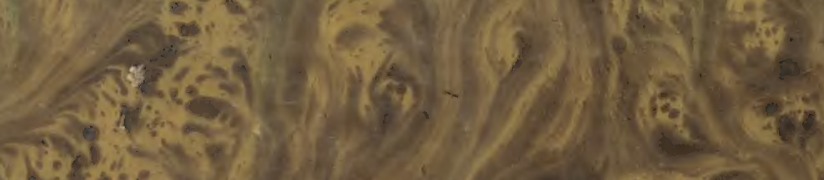

(a) a.

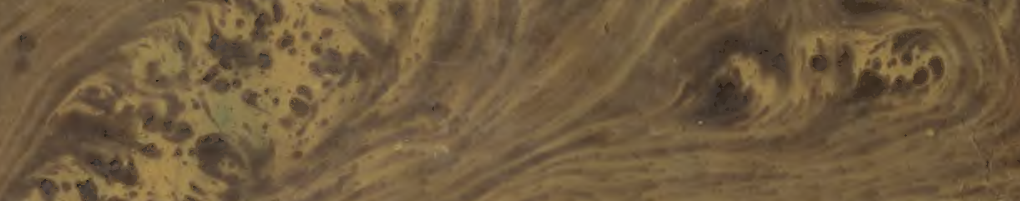

a) 2 (1)

nets?

$(2,8)$

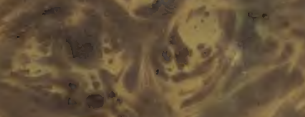

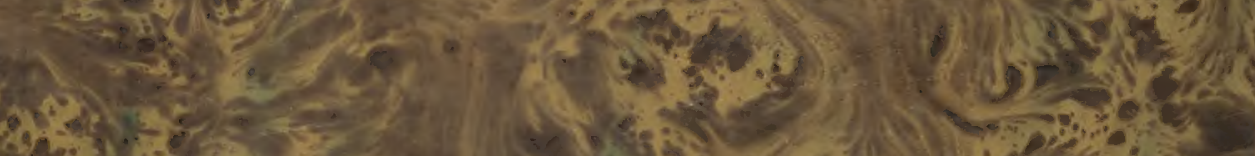

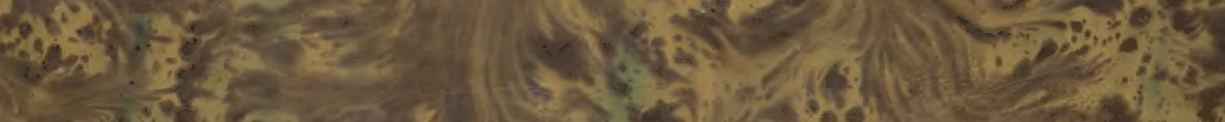

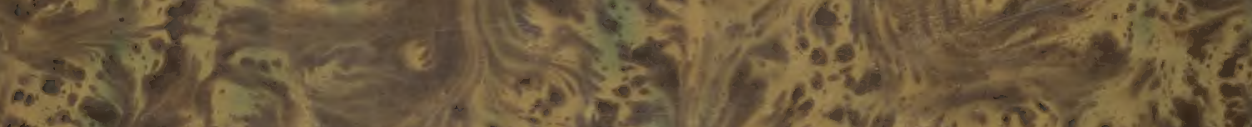

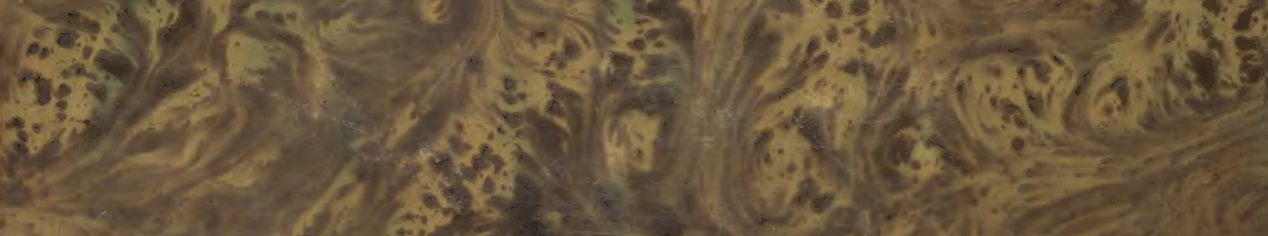

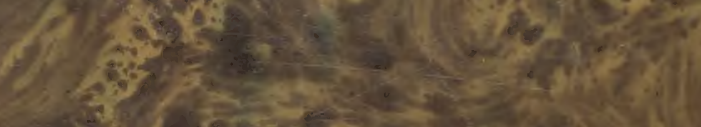

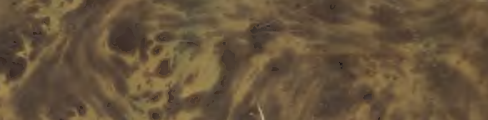

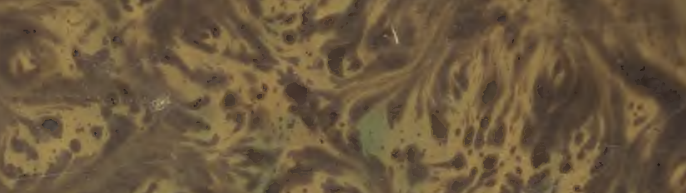

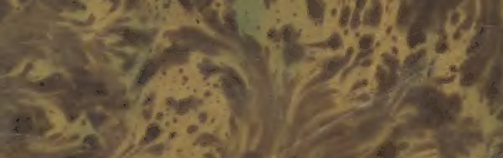

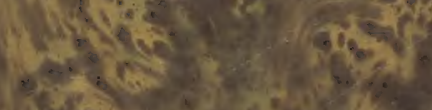

4.

(3.)

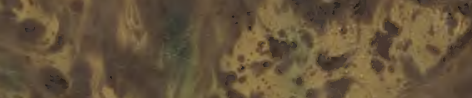

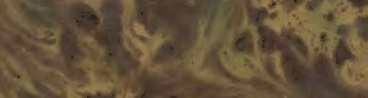

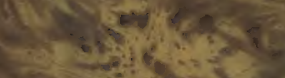

duriflatis
$(-2)$ ase

(i.
(1)

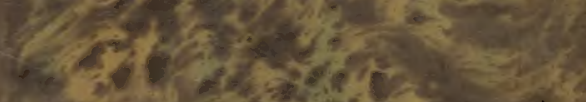
4.

$$
\begin{aligned}
& \text { cisis }
\end{aligned}
$$

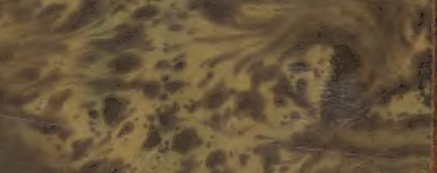

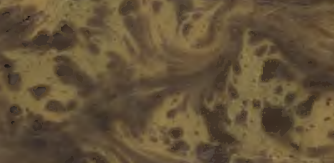

$$
\begin{aligned}
& \text { a) } 5.5
\end{aligned}
$$

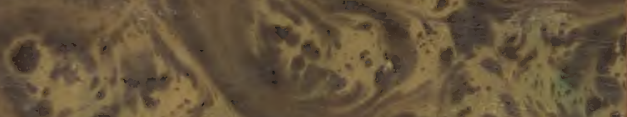

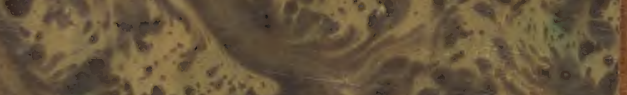

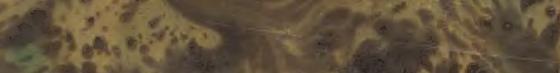

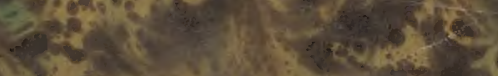

